Universidade DE SÃo PAUlo

Instituto DE FÍsicA

\title{
Vacâncias de Oxigênio e Diluição de Ítrio no Pirocloro Geometricamente Frustrado $\mathrm{Gd}_{2} \mathrm{Ti}_{2} \mathrm{O}_{7}$
}

\author{
Jonathan Gustavo Acosta Ramón
}

Orientador: Prof. Dr. Rafael Sá de Freitas

Dissertação de mestrado apresentada ao Instituto de Física da Universidade de São Paulo para a obtenção do título de

Mestre em Ciências.

\section{Banca Examinadora:}

Prof. Dr. Rafael Sá de Freitas (IF-USP)

Prof. Dr. André de Pinho Vieira (IF-USP)

Prof. Dr. Marcos de Abreu Ávila (UFABC)

São Paulo 
FICHA CATALOGRÁFICA

Preparada pelo Serviço de Biblioteca e Informação do Instituto de Física da Universidade de São Paulo

Acosta Ramón, Jonathan Gustavo

Vacâncias de oxigênio e diluição de ítrio no pirocloro geometricamente frustrado Gd2Ti2O7. São Paulo, 2015.

Dissertação (Mestrado) - Universidade de São Paulo. Instituto de Física. Depto. de Física dos Materiais e Mecânica.

Orientador: Prof. Dr. Rafael Sá de Freitas

Área de Concentração: Fenômenos Magnéticos

Unitermos: 1. Fenômenos magnéticos; 2. Frustração geométrica; 3. Pirocloro; 4. Defeitos cristalinos. 


\section{University of SÃo PAUlo \\ Institute OF Physics}

\section{Oxygen Vacancies and Yttrium Dilution in the Geometrically Frustrated Pyrochlore $\mathrm{Gd}_{2} \mathrm{Ti}_{2} \mathrm{O}_{7}$}

\section{Jonathan Gustavo Acosta Ramón}

Supervisor: Prof. Dr. Rafael Sá de Freitas

Master dissertation submitted to the

Institute of Physics of the

University of São Paulo

for the Degree of Master in Physics

Approved by:

Prof. Dr. Rafael Sá de Freitas (IF-USP)

Prof. Dr. André de Pinho Vieira (IF-USP)

Prof. Dr. Marcos de Abreu Ávila (UFABC)

São Paulo 
for Mrs. Luz 


\section{Acknowledgements}

My deepest gratitude to my supervisor Rafael Sá de Freitas for his patient guidance and the support throughout my studies. I am especially thankful to him for introducing me into the area of highly frustrated magnetism, for working with me in the sessions of lab, and for his kind help outside the academic life.

I would like to thank Prof. Dr. Flavio Vicci and Marina Leite from the Institute of Chemistry for preparing the samples studied in my dissertation and for performing X-ray diffraction experiments, as well as for their help to improve the method of sample preparation.

I am also grateful to Lina, Pablo, Everton, Leandro, and Luciano from the work group of Rafael for helping me at different points of my dissertation.

Thanks to my family for supporting me and to my closest friends Diana, Noemi, Laura, Fernando, Juan, Carlos and Fabiano for their friendship and for being such splendid companions.

To Department of Mechanics and Materials Physics of the IFUSP for their facilities and laboratories, and to CNPq for the scholarship. 


\section{Resumo}

$\mathrm{O}$ composto magnético geometricamente frustrado $\mathrm{Gd}_{2} \mathrm{Ti}_{2} \mathrm{O}_{7}$ da família dos pirocloros apresenta um comportamento bastante interessante, sendo que a natureza da fase magnética em baixas temperaturas se encontra ainda sob intenso debate. Este material entra em um estado antiferromagnético parcialmente ordenado à temperatura $\mathrm{T}_{\mathrm{N}}^{1} \sim 1 \mathrm{~K}$, apresentando outra transição de fase em $\mathrm{T}_{\mathrm{N}}^{2} \sim$ $0.7 \mathrm{~K}$. Neste trabalho é investigada a física de baixas temperaturas de amostras de $\mathrm{Gd}_{2} \mathrm{Ti}_{2} \mathrm{O}_{7}$ com defeitos estruturais tais como vacâncias de oxigênio e diluição de ítrio. Amostras policristalinas com composição $\mathrm{Gd}_{2} \mathrm{Ti}_{2} \mathrm{O}_{7-\delta}$ e $\mathrm{Gd}_{2-\mathrm{x}} \mathrm{Y}_{\mathrm{x}} \mathrm{Ti}_{2} \mathrm{O}_{7}$ foram sintetizadas em diferentes condições por uma rota alternativa conhecida como método sol-gel. O refinamento de um modelo para os dados de difração de raios X mostra que vacâncias de oxigênio são os principais defeitos estruturais neste material. As vacâncias de oxigênio resultam numa ligeira diminuição de $T_{N}^{1}$ e numa redução da magnetização de saturação. A diluição da rede com ítrio leva a uma clara diminuição de $\mathrm{T}_{\mathrm{N}}^{1}$ e da temperatura de Curie-Weiss $\theta_{\mathrm{CW}}$. Medidas de calor específico evidenciaram as duas transições $\mathrm{T}_{\mathrm{N}}^{1}$ e $\mathrm{T}_{\mathrm{N}}^{2}$ no composto com menor grau de vacâncias de oxigênio. A análise da contribuição magnética ao calor específico $C_{m}$ em baixas temperaturas, $0.39 \mathrm{~K} \leq \mathrm{T} \leq 0.68 \mathrm{~K}$, revelou um comportamento proporcional a $\mathrm{T}^{2}$ previamente discutido na literatura. Entretanto, verificamos que uma dependência $C_{m} \propto \mathrm{T}^{3}$, usualmente encontrada em antiferromagnetos convencionais, descreve igualmente bem nossos dados experimentais resultando em uma velocidade de magnons consistente com a apresentada por outros pirocloros.

Palavras-chave: Magnetos Geometricamente Frustrados, Estrutura Pirocloro, Defeitos Estruturais. 


\section{Abstract}

The geometrically frustrated compound $\mathrm{Gd}_{2} \mathrm{Ti}_{2} \mathrm{O}_{7}$ of the pyrochlore family displays such an interesting behaviour that the nature of the ordered magnetic phase at low temperatures is still under intense discussion. This material enters in a partially ordered magnetic state at a temperature $\mathrm{T}_{\mathrm{N}}^{1} \sim 1 \mathrm{~K}$, and there is another phase transition at $\mathrm{T}_{\mathrm{N}}^{2} \sim 0.7 \mathrm{~K}$. In this dissertation we study the low temperature physics of $\mathrm{Gd}_{2} \mathrm{Ti}_{2} \mathrm{O}_{7}$ with structural defects such as oxygen vacancies and yttrium dilution. Polycrystalline samples of $\mathrm{Gd}_{2} \mathrm{Ti}_{2} \mathrm{O}_{7-\delta}$ and $\mathrm{Gd}_{2-\mathrm{x}} \mathrm{Y}_{\mathrm{x}} \mathrm{Ti}_{2} \mathrm{O}_{7}$ were synthesized in different conditions by an alternative route known as the sol-gel method. The refinement of a model for the $\mathrm{X}$-ray diffraction data reveals that the oxygen vacancies are the leading defects in this material. The oxygen vacancies result in a slight decrease of $\mathrm{T}_{\mathrm{N}}^{1}$ and in a reduction of the saturation magnetization. The yttrium dilution of the lattice leads to a clear reduction of $\mathrm{T}_{\mathrm{N}}^{1}$ and of the Curie-Weiss temperature $\theta_{\mathrm{CW}}$. Specific heat measurements display both transitions $\mathrm{T}_{\mathrm{N}}^{1}$ and $\mathrm{T}_{\mathrm{N}}^{2}$ in the compound with lower degree of oxygen vacancies. The analysis of the magnetic contribution to the specific heat $C_{m}$ at low temperatures, $0.39 \mathrm{~K} \leq \mathrm{T} \leq 0.68 \mathrm{~K}$, reveals a behaviour proportional to $\mathrm{T}^{2}$ previously discussed in the literature. However, we verify that a dependence $C_{m} \propto \mathrm{T}^{3}$, usually found in standard antiferromagnets, describes similarly well our experimental data resulting in a velocity of magnons consistent with the ones exhibited for another pyrochlores.

Keywords: Geometrically Frustrated Magnets, Pyrochlore Structure, Structural Defects. 


\section{Contents}

$\begin{array}{ll}\text { Acknowledgements } & \text { ii }\end{array}$

Resumo

Abstract $\quad$ iv

List of Figures $\quad$ viii

List of Tables $\quad$ xiv

$\begin{array}{lll}\text { Chapter } 1 & \text { Introduction } & 1\end{array}$

Chapter 2 Fundamentals and Theoretical Background $\quad 4$

2.1 Magnetic interactions . . . . . . . . . . . . . . . . . . 5

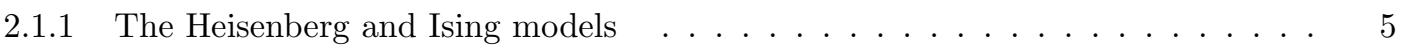

2.1.2 Magnetic dipolar interactions . . . . . . . . . . . . . . . . . 6

2.1.3 Anisotropic exchange interaction . . . . . . . . . . . . . . . . . . 7

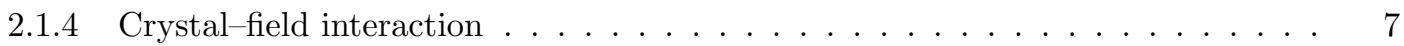

2.1 .5 Single-ion anisotropy $\ldots \ldots \ldots \ldots \ldots \ldots \ldots$

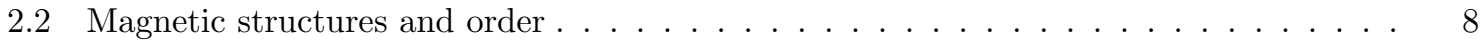

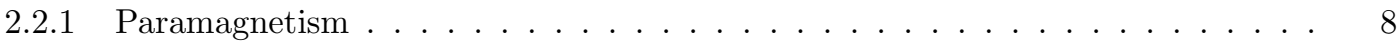

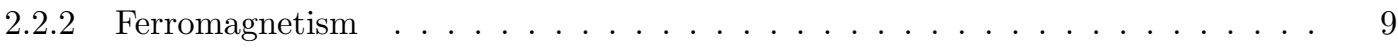

2.2 .3 Antiferromagnetism ............................. 11

2.2 .4 Magnetic susceptibility . . . . . . . . . . . . . . . . . . . 12

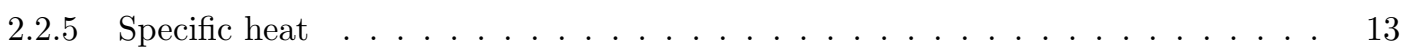

2.3 Geometric frustration $\ldots \ldots \ldots \ldots \ldots \ldots \ldots \ldots$

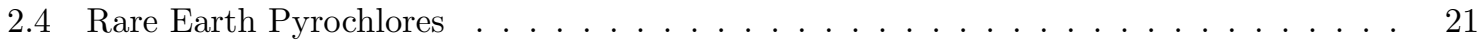


$2.4 .1 \quad$ Pyrochlore structure . . . . . . . . . . . . . . . . . . . . . . 21

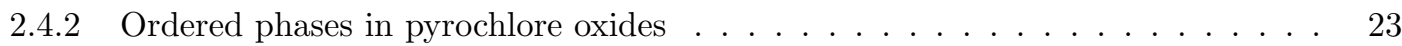

2.5 Gadolinium Titanate $-\mathrm{Gd}_{2} \mathrm{Ti}_{2} \mathrm{O}_{7} \ldots \ldots \ldots \ldots \ldots \ldots \ldots$

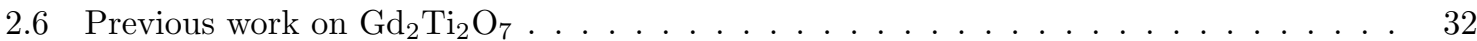

2.6.1 Long-range magnetic order in $\mathrm{Gd}_{2} \mathrm{Ti}_{2} \mathrm{O}_{7} \ldots \ldots \ldots \ldots \ldots \ldots \ldots$

2.6.2 Palmer-Chalker State in the pyrochlore antiferromagnet . . . . . . . . . . . 35

2.6.3 Low-temperature magnetic properties of $\mathrm{Gd}_{2} \mathrm{Ti}_{2} \mathrm{O}_{7} \ldots \ldots \ldots \ldots$. . . . . . 37

2.6.4 Neutron diffraction and multi-k order in $\mathrm{Gd}_{2} \mathrm{Ti}_{2} \mathrm{O}_{7} \ldots \ldots \ldots \ldots$. . . . . . 41

2.6.5 Magnetic ordering in $\mathrm{Gd}_{2} \mathrm{Sn}_{2} \mathrm{O}_{7}$ and $\mathrm{Gd}_{2} \mathrm{Ti}_{2} \mathrm{O}_{7} \ldots \ldots \ldots \ldots$. . . . . . . 44

2.6.6 The low temperature magnetic ground state of $\mathrm{Gd}_{2} \mathrm{Ti}_{2} \mathrm{O}_{7}$ is not $4-k \ldots \ldots$

2.6 .7 Vacancy defects in pyrochlores f . . . . . . . . . . . . . . . 46

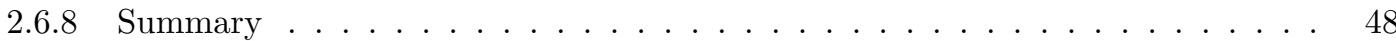

$\begin{array}{lll}\text { Chapter } 3 & \text { Sample Preparation and Experimental Techniques } & 50\end{array}$

3.1 Sol-gel method . . . . . . . . . . . . . . . . . . . . . . . . . . 51

3.1.1 Polycrystalline growth of $\mathrm{Gd}_{2} \mathrm{Ti}_{2} \mathrm{O}_{7} \ldots \ldots \ldots \ldots \ldots \ldots$. . . . . . . . . 51

3.1.2 Polycrystalline growth of $\mathrm{Gd}_{2-x} \mathrm{Y}_{x} \mathrm{Ti}_{2} \mathrm{O}_{7} \ldots \ldots \ldots \ldots \ldots$

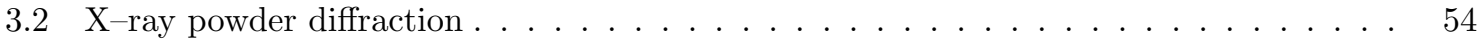

3.3 Magnetic measurements . . . . . . . . . . . . . . . . . . . 56

3.3 .1 Vibrating Sample Magnetometer $(\mathrm{VSM}) \ldots \ldots \ldots \ldots \ldots$

3.3.2 Home-made AC susceptometer . . . . . . . . . . . . . . . . . . . . . . . 58

3.3.3 Superconducting Quantum Interference Device (SQUID) . . . . . . . . . . . . . 60

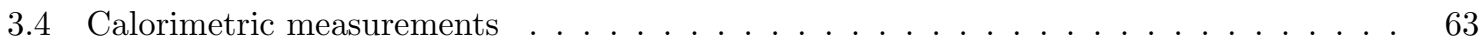

3.4.1 Physical Property Measurement System (PPMS) _ . . . . . . . . . . . . . 63

Chapter 4 Vacancy Defects in Oxygen-deficient $\operatorname{Gd}_{2} \mathbf{T i}_{2} \mathrm{O}_{7} \quad 65$

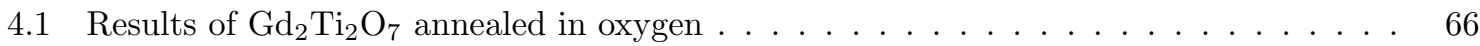

4.1 .1 Structural analysis . . . . . . . . . . . . . . . . . . . . . 66

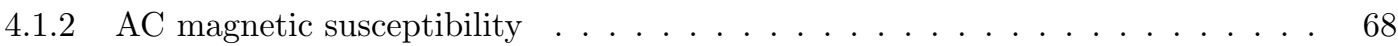

4.1 .3 DC magnetic susceptibility . . . . . . . . . . . . . . . . . . . 70

4.1 .4 Saturation magnetization . . . . . . . . . . . . . . . . . . 73

4.2 Results of $\mathrm{Gd}_{2} \mathrm{Ti}_{2} \mathrm{O}_{7}$ grown in different atmospheres $\ldots \ldots \ldots \ldots \ldots \ldots$ 
4.2 .1 Structural analysis . . . . . . . . . . . . . . . . . . . 75

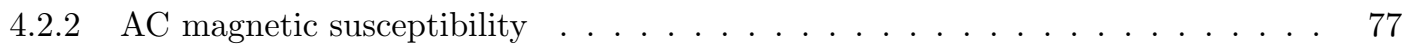

4.2 .3 DC magnetic susceptibility $\ldots \ldots \ldots \ldots \ldots \ldots$

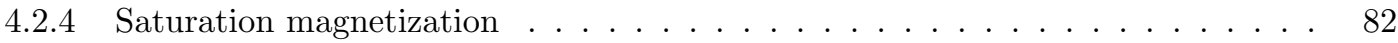

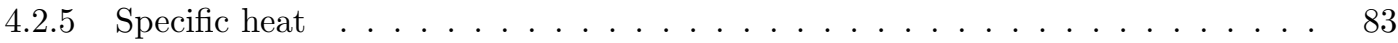

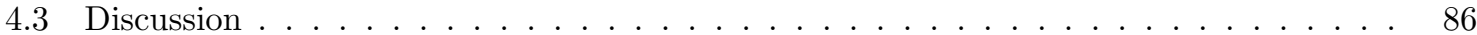

Chapter 5 Yttrium Dilution Effects on $\mathrm{Gd}_{2} \mathbf{T i}_{2} \mathrm{O}_{7} \quad \mathbf{8 9}$

5.1 Results . . . . . . . . . . . . . . . . . . . . . . . . . . 89

5.1 .1 Structural analysis . . . . . . . . . . . . . . . . . . . 90

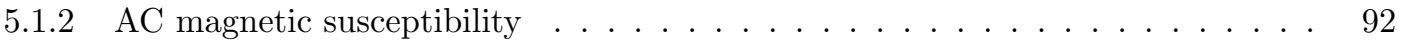

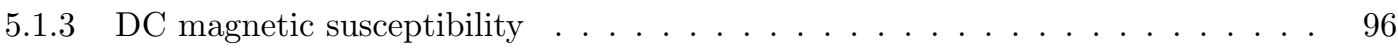

5.1 .4 Saturation magnetization . . . . . . . . . . . . . . . . . . 98

5.1 .5 Specific heat . . . . . . . . . . . . . . . . . . . . . 100

5.2 Discussion . . . . . . . . . . . . . . . . . . . . . . . . . . . 102

$\begin{array}{lll}\text { Chapter } 6 & \text { Conclusion } & 104\end{array}$

List of References $\quad 106$ 


\section{List of Figures}

2.1 Spins on a linear arrangement illustrating its ground state corresponding to antiferromag-

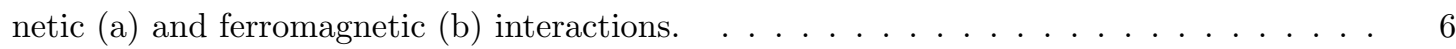

2.2 An antiferromagnet can be considered as two interpenetrating sublattices. . . . . . . . . 11

2.3 Inverse of magnetic susceptibility against temperature graphs for $\theta_{\mathrm{CW}}=0$ (paramagnet), $\theta_{\mathrm{CW}}=\Theta>0$ (ferromagnet) and $\theta_{\mathrm{CW}}=\Theta<0$ (antiferromagnet). This figure was taken

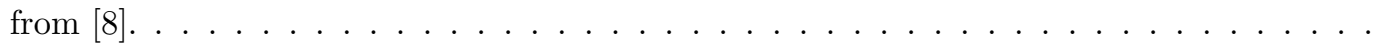

2.4 Spins with antiferromagnetic exchange arranged on a triangle or tetrahedron are geometrically frustrated. In the triangle one-third of the sites are always frustrated, while for the

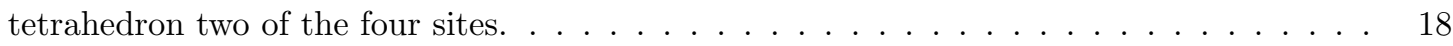

2.5 Heisenberg spins in a triangle lattice are subject to frustration. . . . . . . . . . . . . . 18

2.6 Geometrically frustrated lattices: (a) edge-sharing triangular, (b) corner-sharing triangular or Kagomé, (c) edge-sharing tetrahedral and (d) corner-sharing tetrahedral or pyrochlore. Figure taken from $[7] \ldots \ldots \ldots \ldots \ldots$

2.7 Characteristic behaviour $\chi^{-1}$ vs $\mathrm{T}$ of a geometrically frustrated antiferromagnet (red line). The transition occurs at the temperature $\mathrm{T}_{\mathrm{N}} \ll \theta_{\mathrm{CW}} \ldots \ldots \ldots \ldots \ldots$

2.8 Pyrochlore lattice: the rare-earth ions $\mathrm{A}^{3+}$ of $\mathrm{A}_{2} \mathrm{~B}_{2} \mathrm{O}_{7}$ are located on the vertices of every tetrahedron. The ions $\mathrm{B}^{4+}$ sit on an identical lattice displaced by a translation along the cubic diagonal $[111]$. Figure taken from $[17] \ldots \ldots \ldots \ldots \ldots$

2.9 Ising spins at each vertices of tetrahedron, oriented along local easy-axis $\langle 111\rangle$, and its analogy with the arrangement of protons (white circles) about oxygen in water ice (red circle). The "spin ice" analogy. Figure taken from $[17] \ldots \ldots \ldots \ldots$. . . . . . . . .

$2.10 \mathrm{Dy}_{2} \mathrm{Ti}_{2} \mathrm{O}_{7}$ : (a) Specific heat and (b) entropy as a function of the temperature reported by [24]. The residual entropy of $\mathrm{Dy}_{2} \mathrm{Ti}_{2} \mathrm{O}_{7}, 1.86 \mathrm{~J} . \mathrm{K}^{-1} \cdot \mathrm{mol}^{-1}$, is in agreement with Pauling's entropy, $\frac{R}{2} \ln \left(\frac{3}{2}\right)=1.68 \mathrm{~J} \cdot \mathrm{K}^{-1} \cdot \mathrm{mol}^{-1}[24]$. (a) and (b) also show a comparison with Monte Carlo simulations (MC data) of [25]. This figure was taken from $[24] \ldots$. . . . . . . . 
2.11 Low-temperature specific heat measurements of $\mathrm{Yb}_{2} \mathrm{Ti}_{2} \mathrm{O}_{7}$. A significant sample dependence is observed in both powders and single crystals. The powder sample (blue) shows the highest temperature $(265 \mathrm{mK})$ and sharpest anomaly. The second crystal sample (for neutron scattering purposes), B, shows a sharp peak at $265 \mathrm{mK}$ similar to the powder, and a broad, low-temperature feature similar to crystal A. Figure from [37] . . . . . . . . . .

2.12 (a) The pyrochlore lattice can be visualised as alternating Kagomé and interstitial planar layers stacked along the [111] direction. (b) Corner-sharing tetrahedra of the pyrochlore $\mathrm{Gd}_{2} \mathrm{Ti}_{2} \mathrm{O}_{7}$ with isolated $\mathrm{Gd}$ and $\mathrm{O}$ ions. We have two non-equivalent oxygen at sites $\mathrm{O}(1)$ and $\mathrm{O}(2)$. The Ti ions were removed for clarity. These figures were adapted from [2] and $[52$

2.13 Inverse susceptibility $\chi^{-1}$ of $\mathrm{Gd}_{2} \mathrm{Ti}_{2} \mathrm{O}_{7}$ against temperature in the range $2-25 \mathrm{~K}$, and in the range $2-300 \mathrm{~K}$ in the inset. Figure taken from $[18] \ldots \ldots \ldots \ldots$

2.14 (a) Real part of the ac susceptibility $\chi^{\prime}$ vs temperature at different frequencies, (b) specific heat $\mathrm{C}_{\mathrm{m}}$ vs temperature. The inset displays $\mathrm{C}_{\mathrm{m}}$ in the low-temperature range below $5 \mathrm{~K}$. Figures taken from $[18] \ldots \ldots \ldots \ldots \ldots \ldots$

2.15 The PC state $\mathbf{q}=(000)$ for a single tetrahedron of the pyrochlore lattice. Here, all the spins lie onto the $x y$ plane and are parallel to certain edge of the tetrahedron. Figure taken from $[58] \ldots \ldots \ldots \ldots \ldots \ldots \ldots \ldots \ldots$

2.16 Projection of the $\mathrm{PC}$ state $\mathbf{q}=(000)$ onto the $x y$ plane of the pyrochlore lattice. Figure

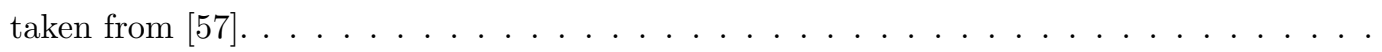

2.17 The ground state $\mathbf{q}=\mathbf{0}$ for spins in the Kagomé lattice. The spins on each triangle order in a $120^{\circ}$ pattern. This figure was taken from $[59] . \ldots \ldots \ldots$. . . . . . . .

2.18 Thermal dependence of the specific heat divided by the temperature of $\mathrm{Gd}_{2} \mathrm{Ti}_{2} \mathrm{O}_{7}$. The inset shows the change in the entropy and the dashed line represents the total magnetic entropy $R \ln (8)=17.3 \mathrm{~J} . \mathrm{K}^{-1} \cdot \mathrm{mol}^{-1}$. This figure was taken from [39]. . . . . . . . . . .

2.19 Specific heat as a function of temperature for $\mathrm{Gd}_{2} \mathrm{Sn}_{2} \mathrm{O}_{7}$. The inset shows the variation in entropy and the dashed lines represent $R \ln (8)=17.3 \mathrm{~J} \cdot \mathrm{K}^{-1} \cdot \mathrm{mol}^{-1} \ldots \ldots \ldots \ldots$

2.20 Spin orientations in the Kagomé planes are perpendicular to crystalline direction [111] and the disordered spins lie in the interstitial sites. The so-called $1-k$ model. Figure from [50]. 
2.21 (a) Temperature dependence of the magnetic susceptibility of $\mathrm{Gd}_{2} \mathrm{Ti}_{2} \mathrm{O}_{7}$ measured with a magnetic field of $100 \mathrm{Oe}$, the arrows indicate the two transitions at 1.02 and $0.74 \mathrm{~K}$. (b) Temperature dependence of the susceptibility measured for different magnetic fields. Figure taken from $[60] \ldots \ldots \ldots \ldots \ldots \ldots \ldots$

2.22 Low temperature magnetic structures $1-k$ and $4-k$ of $\mathrm{Gd}_{2} \mathrm{Ti}_{2} \mathrm{O}_{7}$. In each model the four disordered Gd spins are coloured in orange. The phase transition at $\mathrm{T}_{\mathrm{N}}^{2} \sim 0.75 \mathrm{~K}$ involves partial ordering of these four spins. Figure from $[40] \ldots \ldots \ldots \ldots$

2.23 (a) $1-k$ structure along the direction [111], the ordered sites (blue) form a $120^{\circ}$ pattern on a triangle in a Kagomé plane and the disordered spins lie on the interstitial sites (yellow). (b) $4-k$ structure with the ordered spins being perpendicular to the local $\langle 111\rangle$ axes. Figure taken from $[62] \ldots \ldots \ldots \ldots \ldots \ldots \ldots \ldots$

2.24 Magnetic diffuse scattering at $46 \mathrm{mK}$ (black circles). Fits of the data with 1- $k$ (a) and $4-k$ (b) models. This figure was taken from $[40] \ldots \ldots \ldots \ldots \ldots$

2.25 (a) Pyrochlore lattice of corner-sharing tetrahedra where next-neighbour exchanges are shown by dashed lines. (b) Ordering wavevectors for different values of second- and thirdneighbour exchange constants for a Heisenberg antiferromagnet with dipolar interactions. Figures from $[4] . \ldots \ldots \ldots \ldots \ldots \ldots \ldots$

2.26 Ordering vectors at $\mathrm{T}_{\mathrm{N}}^{1}=1 \mathrm{~K}$ obtained from MFT [62]. The combined area $1-k$ and $4-k$ correspond to the order vector $\mathbf{k}=\left(\frac{1}{2} \frac{1}{2} \frac{1}{2}\right)$. The $\mathbf{k}=\mathbf{0}$ region involve all states for which all spins are fully ordered. Figure taken from $[62] \ldots \ldots \ldots \ldots$

2.27 Defect structure in the pyrochlore $\mathrm{Y}_{2} \mathrm{Ti}_{2} \mathrm{O}_{7-\delta}$ around an $\mathrm{O}(1)$ vacancy defect. The displacements are displayed by green arrows. Figure adapted from $[63] \ldots \ldots \ldots \ldots$. . . .

3.1 Diagram of the diffractometer. This figure was taken from [69] . . . . . . . . . . . . .

3.2 Diagram of the diffractometer. The diffraction angle, $2 \theta$, and the incident angle, $\omega$, which is the half of the diffraction angle $2 \theta$. Figure from $[69] \ldots \ldots \ldots \ldots$

3.3 (a) Diagram of the vibrating sample magnetometer (VSM). (b) Detailed mechanical features of the VSM. This figure was adapted from [73] . . . . . . . . . . . . 56

3.4 Schematic diagram of the VSM inside the pumped helium-4 cryostat. . . . . . . . . . 58

3.5 Primary and secondary coil configuration. Figure taken from [74] . . . . . . . . . . 59

3.6 Diagram showing the Josephson junctions (denoted X), and the superconducting ring. $\Phi$ is the magnetic flux through the superconducting ring and $\mathrm{I}$ is the current through the loop. 60 
3.7 Diagram of detection of the SQUID. This figure was adapted from [75]. . . . . . . . . 62

3.8 Sample and sample platform in the PPMS. This figure was taken from [78] . . . . . . . 63

4.1 Powder X-ray diffraction (red points) and calculated (black solid line) data for $\mathrm{Gd}_{2} \mathrm{Ti}_{2} \mathrm{O}_{7}$ annealed in oxygen. The Rietveld refinement using Fullprof software was performed with the X-ray diffraction pattern of $\mathrm{Gd}_{2} \mathrm{Ti}_{2} \mathrm{O}_{7}$ measured at room temperature. . . . . . . .

4.2 ac magnetic susceptibility $\chi_{a c}$ as a function of the temperature for the as-grown, first annealing and second annealing in oxygen $\mathrm{Gd}_{2} \mathrm{Ti}_{2} \mathrm{O}_{7}$ samples measured at zero field. . . .

4.3 Left: Full $\chi_{a c}$ data between $1 \mathrm{~K}$ and $20 \mathrm{~K}$ for the as-grown, first annealing and second annealing in oxygen $\mathrm{Gd}_{2} \mathrm{Ti}_{2} \mathrm{O}_{7}$ samples at zero field. The $\chi_{a c}$ curves are slightly offset for clarity. Right: $\chi_{a c}$ data between 1 and $30 \mathrm{~K}$ without the offset. . . . . . . . . . . .

$4.4 \mathrm{dc}$ magnetic susceptibility $\chi$ for the as-grown, first annealing and second annealing $\mathrm{Gd}_{2} \mathrm{Ti}_{2} \mathrm{O}_{7}$ samples measured with a field $H=1000$ e. The $\chi$ curves are slightly offset for clarity. . .

4.5 Inverse magnetic susceptibility $\chi^{-1}$ as a function of the temperature and its respective linear fit of Curie-Weiss for the as-grown (blue), first annealing (red) and second annealing

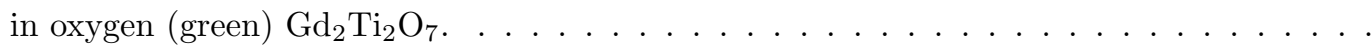

4.6 Inverse magnetic susceptibility $\chi^{-1}$ in the temperature range of $2.5-22.5 \mathrm{~K}$ for the asgrown, first annealing and second annealing in oxygen $\mathrm{Gd}_{2} \mathrm{Ti}_{2} \mathrm{O}_{7}$ samples. The $\chi^{-1}$ curves and the linear fits are shown slightly offset for clarity. . . . . . . . . . . . . .

4.7 Saturation magnetization as a function of the magnetic field for the as-grown $\mathrm{Gd}_{2} \mathrm{Ti}_{2} \mathrm{O}_{7}$ (blue) and for $\mathrm{Gd}_{2} \mathrm{Ti}_{2} \mathrm{O}_{7}$ with a first (red) and second (green) annealing in oxygen at $\mathrm{T}=$ $1.26 \mathrm{~K}$. Inset, magnetization as a function of the magnetic field for the $\mathrm{Gd}_{2} \mathrm{Ti}_{2} \mathrm{O}_{7}$ samples

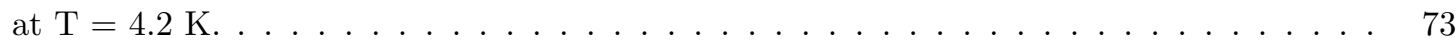

4.8 Powder X-ray diffraction patterns for $\mathrm{Gd}_{2} \mathrm{Ti}_{2} \mathrm{O}_{7}$ grown in air, $\mathrm{H}_{2}$, and $\mathrm{O}_{2} \ldots \ldots \ldots$

4.9 Temperature dependence of the ac magnetic susceptibility $\chi_{a c}$ for $\mathrm{Gd}_{2} \mathrm{Ti}_{2} \mathrm{O}_{7}$ grown in different atmospheres: air (blue), $\mathrm{H}_{2}$ (purple), and $\mathrm{O}_{2}$ (red) measured at zero field. . . . .

4.10 Left: Full data of ac magnetic susceptibility $\chi_{a c}$ as a function of the temperature for $\mathrm{Gd}_{2} \mathrm{Ti}_{2} \mathrm{O}_{7}$ grown in air, $\mathrm{H}_{2}$, and $\mathrm{O}_{2}$ measured at zero field. $\chi_{a c}$ curves present a slight offset for clarity. Right: Raw $\chi_{a c}$ data of the $\mathrm{Gd}_{2} \mathrm{Ti}_{2} \mathrm{O}_{7}$ samples. . . . . . . . . . . . .

4.11 Field dependence of the ac susceptibility $\chi_{a c}(\mathrm{H})$ for $\mathrm{Gd}_{2} \mathrm{Ti}_{2} \mathrm{O}_{7}$ grown in oxygen atmosphere at temperatures above and below the transition temperature $\mathrm{T}_{\mathrm{N}}^{1}=1.02 \mathrm{~K}$. The $0.50,0.90$, and $1.11 \mathrm{~K}$ data are offset by $+3.5,-3.0$, and $-5.0 \mathrm{emu} / \mathrm{mol}-\mathrm{Oe}$, respectively, for clarity. . 
4.12 Inverse susceptibility $\chi^{-1}$ as a function of the temperature and its respective linear fit of Curie-Weiss for $\mathrm{Gd}_{2} \mathrm{Ti}_{2} \mathrm{O}_{7}$ grown in air (blue), $\mathrm{H}_{2}$ (purple) and $\mathrm{O}_{2}$ (red) measured in an

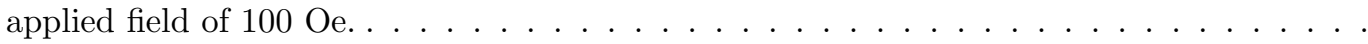

4.13 Inverse magnetic susceptibility $\chi^{-1}$ between $2 \mathrm{~K}$ and $22.5 \mathrm{~K}$ for $\mathrm{Gd}_{2} \mathrm{Ti}_{2} \mathrm{O}_{7}$ grown in $\mathrm{H}_{2}$,

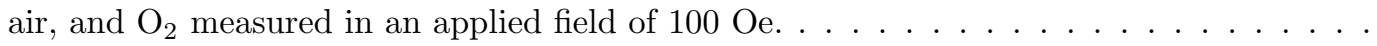

4.14 Saturation magnetization as a function of the applied field for $\mathrm{Gd}_{2} \mathrm{Ti}_{2} \mathrm{O}_{7}$ grown in air, $\mathrm{H}_{2}$, and $\mathrm{O}_{2}$ measured at $4.2 \mathrm{~K}$. Dash line indicates the expected theoretical value of $7 \mu_{B} / \mathrm{Gd}$

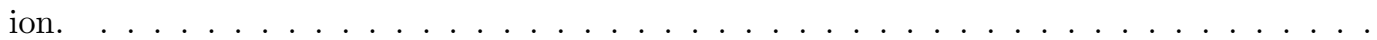

4.15 Temperature dependence of the magnetic specific heat divided by the temperature $C_{m}(\mathrm{~T}) / \mathrm{T}$ for $\mathrm{Gd}_{2} \mathrm{Ti}_{2} \mathrm{O}_{7}$ measured in zero field. Two phase transitions occur at $\mathrm{T}_{\mathrm{N}}^{1}=1.04 \mathrm{~K}$ and $\mathrm{T}_{\mathrm{N}}^{2}$ $=0.73 \mathrm{~K}$. The inset displays the entropy variation as a function of the temperature. . . .

4.16 Magnetic specific heat $C_{m}$ of $\mathrm{Gd}_{2} \mathrm{Ti}_{2} \mathrm{O}_{7}$ as a function of the temperature below the second transition $\mathrm{T}_{\mathrm{N}}^{2}=0.73 \mathrm{~K}$. The $\mathrm{T}^{2}$ power law previously proposed and a $\mathrm{T}^{3}$ power law are

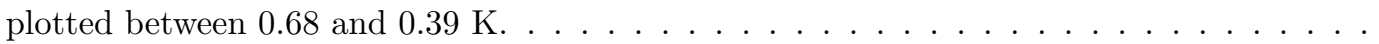

4.17 Temperature dependence of the magnetic specific heat $C_{m}$ of $\mathrm{Gd}_{2} \mathrm{Ti}_{2} \mathrm{O}_{7}$ measured with different magnetic fields. All the $C_{m}$ curves present a Schottky anomaly above the transition temperature $\mathrm{T}_{\mathrm{N}}^{1}=1.04 \mathrm{~K}$. The solid lines represent the fits of the data to the Schottky specific heat. . . . . . . . . . . . . . . . . . . . . . . .

5.1 Powder X-ray diffraction patterns at room temperature, obtained from $\operatorname{Gd}_{2-\mathrm{x}} \mathrm{Y}_{\mathrm{x}} \operatorname{Ti}_{2} \mathrm{O}_{7}$ samples with different yttrium contents $\mathrm{x}$. The diffraction data is offset for clarity. . . . . 90

5.2 Refined lattice parameter $( \pm 0.02 \%)$ as a function of the yttrium content x. . . . . . . 91

5.3 ac magnetic susceptibility $\chi_{a c}$ as a function of the temperature for $\operatorname{Gd}_{2-\mathrm{x}} \mathrm{Y}_{\mathrm{x}} \mathrm{Ti}_{2} \mathrm{O}_{7}$ with different yttrium contents $\mathrm{x}$ measured at zero field. $\chi_{a c}$ curves are slight offset for clarity.

5.4 Left: Temperature dependence of the ac magnetic susceptibility $\chi_{a c}$ of $\mathrm{Gd}_{2-\mathrm{x}} \mathrm{Y}_{\mathrm{x}} \mathrm{Ti}_{2} \mathrm{O}_{7}$ for $\mathrm{x}=0-0.7$. The $\chi_{a c}$ curves are slightly offset for clarity. Right: Raw data for the ac magnetic susceptibility of $\mathrm{Gd}_{2-\mathrm{x}} \mathrm{Y}_{\mathrm{x}} \mathrm{Ti}_{2} \mathrm{O}_{7} \ldots \ldots \ldots \ldots \ldots \ldots \ldots$

5.5 Transition temperatures $\mathrm{T}_{\mathrm{N}}^{1}$ as a function of the yttrium content $\mathrm{x}$ in $\operatorname{Gd}_{2-\mathrm{x}} \mathrm{Y}_{\mathrm{x}} \mathrm{Ti}_{2} \mathrm{O}_{7}$. . . 94

5.6 Field dependence of ac susceptibility for $\mathrm{Gd}_{2-\mathrm{x}} \mathrm{Y}_{\mathrm{x}} \mathrm{Ti}_{2} \mathrm{O}_{7}$ with yttrium content $\mathrm{x}=0.3$

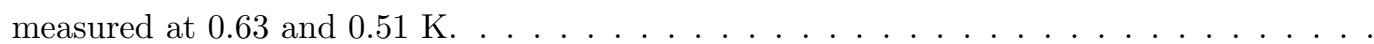

5.7 Inverse susceptibility $\chi^{-1}$ as a function of the temperature and its respective linear fit of Curie-Weiss for $\mathrm{Gd}_{2-\mathrm{x}} \mathrm{Y}_{\mathrm{x}} \mathrm{Ti}_{2} \mathrm{O}_{7}$ measured in an applied field of 100 Oe. . . . . . . . . 96 
5.8 Curie-Weiss temperatures $\left|\theta_{\mathrm{CW}}\right|( \pm 5 \%)$ (left axis) and effective magnetic moments $\mathrm{p}_{\text {eff }}$ $( \pm 1 \%)$ (right axis) for $\mathrm{Gd}_{2-\mathrm{x}} \mathrm{Y}_{\mathrm{x}} \mathrm{Ti}_{2} \mathrm{O}_{7}$ as a function of the yttrium content $\mathrm{x} . \quad \ldots \ldots . \quad \ldots 7$

5.9 Saturation magnetization for $\mathrm{Gd}_{2-\mathrm{x}} \mathrm{Y}_{\mathrm{x}} \mathrm{Ti}_{2} \mathrm{O}_{7}$ with different yttrium concentrations $\mathrm{x}$ measured at 1.28 and $4.2 \mathrm{~K} \ldots \ldots \ldots \ldots \ldots \ldots \ldots$

5.10 Magnetization as a function of the applied field of $\mathrm{Gd}_{2-\mathrm{x}} \mathrm{Y}_{\mathrm{x}} \mathrm{Ti}_{2} \mathrm{O}_{7}$ with $\mathrm{x}=1,1.1$ and 1.5 measured at $4.2 \mathrm{~K} \ldots \ldots \ldots \ldots \ldots \ldots$

5.11 Temperature dependence of the total specific heat $C$ for $\mathrm{Gd}_{2-\mathrm{x}} \mathrm{Y}_{\mathrm{x}} \mathrm{Ti}_{2} \mathrm{O}_{7}$ with different yttrium contents $\mathrm{x}$ measured in zero field. . . . . . . . . . . . . . . . . . . . 100

5.12 Magnetic specific heat $C_{m}$ as a function of the temperature for $\mathrm{Gd}_{2-\mathrm{x}} \mathrm{Y}_{\mathrm{x}} \mathrm{Ti}_{2} \mathrm{O}_{7}$ with yttrium contents $\mathrm{x}=0.1$ (left) and $\mathrm{x}=0.4$ (right) measured with different magnetic fields. . . . 101

5.13 Magnetic specific heat $C_{m}$ as a function of the temperature for $\mathrm{Gd}_{2-\mathrm{x}} \mathrm{Y}_{\mathrm{x}} \mathrm{Ti}_{2} \mathrm{O}_{7}$ with yttrium contents $\mathrm{x}=0.7$ (left) and $\mathrm{x}=1$ (right) measured with different magnetic fields. . . . . 102 


\section{List of Tables}

2.1 The crystallographic positions for the space group $F d \overline{3} m$ suitable for the cubic pyrochlore

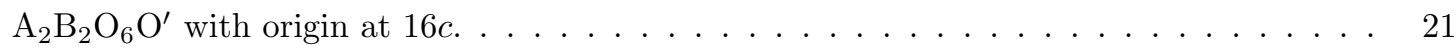

2.2 Presence or absence of long-range order in the Ti-based pyrochlores. . . . . . . . . . . . 29

4.1 Refinement of the structure of $\mathrm{Gd}_{2} \mathrm{Ti}_{2} \mathrm{O}_{7}$ annealed in oxygen showing the space group, the lattice parameter, the occupancies of each site, and the value of chi-squared. . . . . . . .

4.2 Transition temperatures, $\mathrm{T}_{\mathrm{N}}^{1}$, for $\mathrm{Gd}_{2} \mathrm{Ti}_{2} \mathrm{O}_{7}$ as-grown, with a first annealing in oxygen, and with a second annealing in oxygen. . . . . . . . . . . . . . . . 70

4.3 Values of the Curie-Weiss temperatures $\theta_{\mathrm{CW}}$, the effective magnetic moments $\mathrm{p}_{\text {eff }}$, the transition temperatures $\mathrm{T}_{\mathrm{N}}^{1}$, and the frustration indexes $f$ for the oxygen-deficient samples

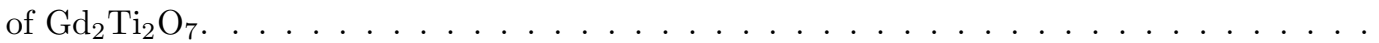

4.4 Refinement of the structure of $\mathrm{Gd}_{2} \mathrm{Ti}_{2} \mathrm{O}_{7}$ grown in air, $\mathrm{H}_{2}$, and $\mathrm{O}_{2}$ showing the space groups, the lattice parameters, the occupancies of each site, and the values of chi-squared. $\quad 76$

4.5 Transition temperatures $\mathrm{T}_{\mathrm{N}}^{1}$ for $\mathrm{Gd}_{2} \mathrm{Ti}_{2} \mathrm{O}_{7}$ samples grown in $\mathrm{H}_{2}$, air, and $\mathrm{O}_{2} \ldots \ldots$

4.6 Values of the Curie-Weiss temperatures $\theta_{\mathrm{CW}}$, the effective magnetic moments $\mathrm{p}_{\text {eff }}$, the transition temperatures $\mathrm{T}_{\mathrm{N}}^{1}$, and the frustration indexes $f$ for $\mathrm{Gd}_{2} \mathrm{Ti}_{2} \mathrm{O}_{7}$ grown in $\mathrm{H}_{2}$, air,

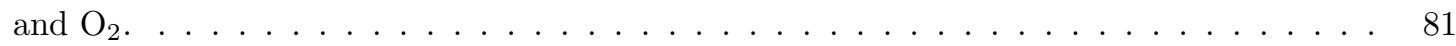

5.1 Refinement of the structure of $\mathrm{Gd}_{2-\mathrm{x}} \mathrm{Y}_{\mathrm{x}} \mathrm{Ti}_{2} \mathrm{O}_{7}$ with different yttrium contents $\mathrm{x} . \ldots \ldots 1$

5.2 Values of the transition temperatures $\mathrm{T}_{\mathrm{N}}^{1}$ for the yttrium diluted $\mathrm{Gd}_{2-\mathrm{x}} \mathrm{Y}_{\mathrm{x}} \mathrm{Ti}_{2} \mathrm{O}_{7} \ldots \ldots$

5.3 Values of the effective magnetic moment, $\mathrm{p}_{\text {eff }}$, Curie-Weiss temperatures, $\theta_{\mathrm{CW}}$, and transition temperatures, $\mathrm{T}_{\mathrm{N}}^{1}$, for different yttrium concentrations $\mathrm{x}$ in $\mathrm{Gd}_{2-\mathrm{x}} \mathrm{Y}_{\mathrm{x}} \mathrm{Ti}_{2} \mathrm{O}_{7} \ldots \ldots$. . 97 


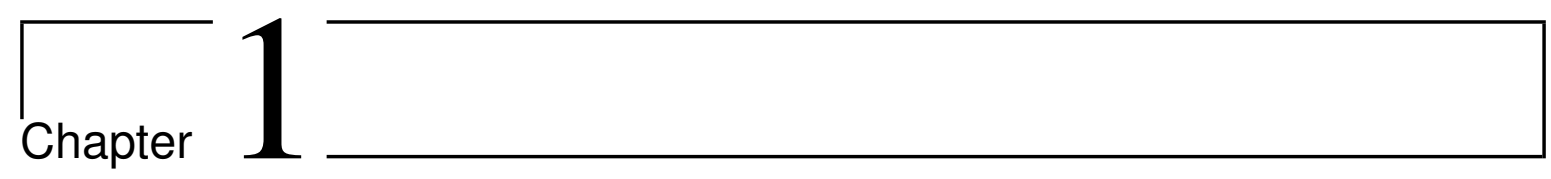

\section{Introduction}

Advances in topics such as magnetism and magnetic materials have taken part in the developing of new technologies for everyday life. For example, applications of magnetism include from hard disk data storage, magnetic resonance imaging, to future public transportation. Indeed, magnetism on the atomic scale is becoming essential as data-storage and modern devices are being miniaturized. So, to continue with the improvement of new technologies, it is indispensable to study and to synthesize new magnetic materials.

In recent years, as a result of several experiments concerning novel materials known as geometrically frustrated magnets it was found that they are subject to new exotic physical properties at microscopic scale. These phenomena occur at very low temperatures and give rise to unconventional quantum mechanical behaviours which are experimental and theoretical challenges. Therefore, in order to describe such magnetic systems it is required to improve the current theoretical models and to extend the experimental perspectives for a better understanding of this new physics.

An active area of research in condensed matter physics is the study of frustrated materials. Systems with their components interacting by competing interactions are subject to frustration. Because of this competition the system cannot minimize the energy of all interactions simultaneously or, in other words, the system cannot find a unique ground state. Also, when those interactions are of similar magnitude, it leads to a large degeneracy of ground states [1]. These attributes made frustration a crucial element in diverse topics of condensed matter physics. Superconducting Josephson

junction arrays [2], liquid crystals [2], and quantum dots (artificial atoms) as potential quantum bits for quantum computation [3] are a few examples of the richness of frustration. It has an important 
role also outside the field of condensed matter physics with a wide range of phenomena such as the folding of proteins and neural networks $[4,5]$.

Geometric frustration occurs in systems where the competition of interactions originates in the spatial arrangement of their components [1]. Two simple and illustrative examples are the magnetic systems whose spins lie on the vertices of an equilateral triangle or a tetrahedron. They are subject to geometric magnetic frustration as we will see in Chapter 2. Other examples of geometric frustration are the magnetic systems with their spins residing on lattices of corner-sharing triangles (Kagomé lattice) or corner-sharing tetrahedra (pyrochlore lattice). Magnetic materials with the mentioned corner-sharing tetrahedra or pyrochlore structure are expected to be ideal candidates for exhibiting large quantum mechanical spin fluctuations and for giving rise to novel magnetic ground states [2].

Experimental realizations of the pyrochlore oxides, $\mathrm{A}_{2} \mathrm{~B}_{2} \mathrm{O}_{7}$, have been essential to go forward in the understanding of geometrically frustrated magnetism. In these oxides, the A and B ions are located on two distinct interpenetrating pyrochlore lattices. The geometrical constraints imposed by the arrangement of the interpenetrating tetrahedra frustrate the near-neighbour magnetic interactions, originating exotic behaviours in the simplest and more complex spin Hamiltonians [6].

In order to study real systems, one must include extra interactions into the simplest spin Hamiltonian which is the nearest-neighbour exchange Hamiltonian $\mathcal{H}=-\sum \mathrm{J}_{i j} \boldsymbol{S}_{i} \cdot \boldsymbol{S}_{j}$. Examples of additional physical contributions are: second or higher neighbour exchanges, dipolar interactions, applied magnetic fields, anisotropic exchange (Dzyaloshinskii-Moriya), thermal or quantum fluctuations, etc [7]. All these interactions compete between them to establish spin-spin correlations as the system reaches extremely low temperatures [2]. The nature of the material being studied determines whether the additional terms in the Hamiltonian lead to a long-range ordered state in the system. Because of that, a fairly high number of pyrochlore oxides display unusual magnetic ground states. Those $\mathrm{A}_{2} \mathrm{~B}_{2} \mathrm{O}_{7}$ materials display a broad range of phenomena comprehending spin glass freezing in $\mathrm{Y}_{2} \mathrm{Mo}_{2} \mathrm{O}_{7}$, spin-liquid behaviour in $\mathrm{Tb}_{2} \mathrm{Ti}_{2} \mathrm{O}_{7}$, spin-ice phenomenon in $\mathrm{Ho}_{2} \mathrm{Ti}_{2} \mathrm{O}_{7}$ and $\mathrm{Dy}_{2} \mathrm{Ti}_{2} \mathrm{O}_{7}, \mathrm{XY}$ antiferromagnetism in $\mathrm{Er}_{2} \mathrm{Ti}_{2} \mathrm{O}_{7}$, and superconductivity in $\mathrm{Cd}_{2} \mathrm{Re}_{2} \mathrm{O}_{7}$, among others [2].

Besides the pyrochlore materials mentioned above, there are other examples being discussed through recent research. For making an improvement in the understanding of the pyrochlore systems this dissertation deals with the phenomena involving $\mathrm{Gd}_{2} \mathrm{Ti}_{2} \mathrm{O}_{7}$, a remarkable example of pyrochlore in which the magnetic ground state involves a partial ordering of spins that is still an open topic.

In Chapter 2, we will review the fundamentals of the magnetic interactions in spin systems. Then, we will explain how frustration is involved with magnetic systems. Moreover, we will study the 
crystal structures of pyrochlore materials and we will explain briefly the exotic magnetic behaviours of spin ice, spin liquid and long-range order. We will review the theoretical and experimental results concerning the magnetic structure at low temperatures for $\mathrm{Gd}_{2} \mathrm{Ti}_{2} \mathrm{O}_{7}$. On the theoretical side, we will study results from mean field theory, but we will not enter on a rigorous formalism because it is not the focus of this dissertation. On the experimental side, we will study results from magnetic, thermal, and neutron diffraction studies to understand the proposed ground state of $\mathrm{Gd}_{2} \mathrm{Ti}_{2} \mathrm{O}_{7}$.

In Chapter 3, we will explain the sample preparation employed and also the experimental techniques used in the laboratory. First, we will describe the alternative chemical method to prepare polycrystalline $\mathrm{Gd}_{2} \mathrm{Ti}_{2} \mathrm{O}_{7}$ known as sol-gel. Then, we will extend this method to prepare $\mathrm{Gd}_{2} \mathrm{Ti}_{2} \mathrm{O}_{7}$ samples with yttrium, that is, samples of formula $\mathrm{Gd}_{2-x} \mathrm{Y}_{x} \mathrm{Ti}_{2} \mathrm{O}_{7}$, where $\mathrm{x}$ is the yttrium proportion. The experiments performed to study the pyrochlore $\mathrm{Gd}_{2} \mathrm{Ti}_{2} \mathrm{O}_{7}$ are magnetization, ac magnetic susceptibility, and specific heat measurements.

In Chapter 4, we will discuss how the long-range magnetic ordering of the pyrochlore $\mathrm{Gd}_{2} \mathrm{Ti}_{2} \mathrm{O}_{7}$ changes due to lattice defects such as oxygen vacancies. Those defects are introduced by employing the mentioned sol-gel method. After this method, we annealed our samples in different atmospheres as oxygen, $\mathrm{O}_{2}$, hydrogen, $\mathrm{H}_{2}$, and air in order to modify the content of the different components of $\mathrm{Gd}_{2} \mathrm{Ti}_{2} \mathrm{O}_{7}$. The lattice defects are important in the magnetic lattice of Gd since we do not know if they will favor the magnetic ordering or not, and as a result a transition to a long-range ordered phase. To verify if our samples were grown with defects of vacancy, we will analyse the $\mathrm{X}$-ray diffraction data by the method called Rietveld refinement using the FullProf software. Then, we will report magnetization, ac magnetic susceptibility, and specific heat data to compare the magnetic properties of the different $\mathrm{Gd}_{2} \mathrm{Ti}_{2} \mathrm{O}_{7}$ samples. Finally, we will discuss if those defects involve a pattern or tendency in the variation of the magnetic properties experimentally measured of $\mathrm{Gd}_{2} \mathrm{Ti}_{2} \mathrm{O}_{7}$.

In Chapter 5, we will study $\mathrm{Gd}_{2} \mathrm{Ti}_{2} \mathrm{O}_{7}$ at low temperatures when it is diluted by yttrium impurities resulting on $\mathrm{Gd}_{2-x} \mathrm{Y}_{x} \mathrm{Ti}_{2} \mathrm{O}_{7}$. We will analyse the $\mathrm{X}$-ray diffraction data to discuss the introduction of yttrium on the magnetic lattice. Also, we will report magnetization, ac magnetic susceptibility, and specific heat of $\mathrm{Gd}_{2-x} \mathrm{Y}_{x} \mathrm{Ti}_{2} \mathrm{O}_{7}$ with different contents of yttrium. We will close with a discussion about the magnetic and thermal properties obtained for the $\mathrm{Gd}_{2-x} \mathrm{Y}_{x} \mathrm{Ti}_{2} \mathrm{O}_{7}$ samples at very low temperatures.

Finally, in Conclusions we will sum up the most important results of this dissertation and also we will mention the future work concerning different experiments to complete our study of $\mathrm{Gd}_{2} \mathrm{Ti}_{2} \mathrm{O}_{7}$ with structural defects. 


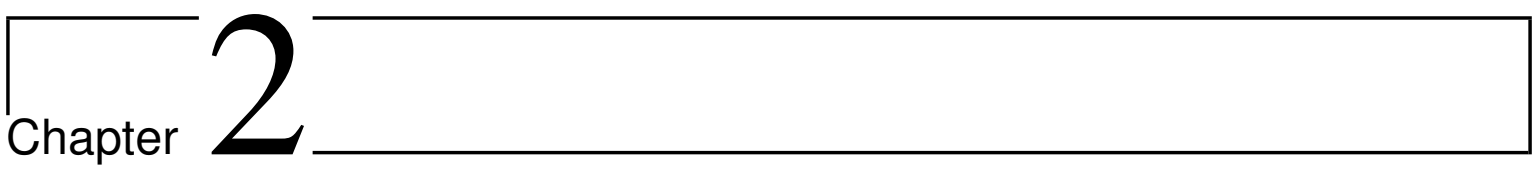

\section{Fundamentals and Theoretical Background}

This chapter is about the fundamentals of magnetism in condensed matter and an introduction to our study of the pyrochlore $\mathrm{Gd}_{2} \mathrm{Ti}_{2} \mathrm{O}_{7}$. We present first the basic theory of magnetic interactions focusing on nearest-neighbour interactions model by the Heisenberg Hamiltonian and additional interaction terms to this Hamiltonian. We deal with the order structures and magnetic properties such as magnetic susceptibility arising from ferromagnetic and antiferromagnetic interactions. We also study the phonon, electron, and magnon contributions to the low temperature specific heat of a solid.

Then, we define the concept of geometrical frustration and its implications on magnetism. We discuss the microscopic properties of geometrically frustrated materials and present typical experimental behaviours to recognize them. We provide examples of geometrically frustrated lattices and among them the pyrochlore lattice which is of our interest.

Next, we focus on the pyrochlore materials $\mathrm{A}_{2} \mathrm{~B}_{2} \mathrm{O}_{7}$, where $\mathrm{A}$ is a rare-earth ion, $\mathrm{B}$ a non-magnetic ion, and $\mathrm{O}$ oxygen. We study the crystal structure of the pyrochlore lattice which consists of arrays of corner-sharing tetrahedra. For pyrochlores with titanium as the B ion, we describe the exotic magnetic ground states induced by geometrical frustration such as long-range order, spin ice, and spin liquid.

Finally, we study the physical properties of the rare-earth pyrochlore $\mathrm{Gd}_{2} \mathrm{Ti}_{2} \mathrm{O}_{7}$ and present previous results taken from magnetic, thermal, and neutron diffraction measurements, which have been pivotal to propose the low-temperature ground state of the pyrochlore antiferromagnet $\mathrm{Gd}_{2} \mathrm{Ti}_{2} \mathrm{O}_{7}$. 


\subsection{Magnetic interactions}

In this dissertation we will look into the low temperature magnetic ordering of the pyrochlore $\mathrm{Gd}_{2} \mathrm{Ti}_{2} \mathrm{O}_{7}$, focusing on its physical properties at very low temperatures. We will therefore begin by introducing the classical Heisenberg model and the Ising model which are two models widely used to describe magnetic spin systems as pyrochlore materials. Having studied both models, we will then spread out the concept of geometric frustration mentioned in the Introduction of this dissertation.

\subsubsection{The Heisenberg and Ising models}

In order to understand the magnetic behaviour of solids, we need to provide microscopic models of the magnetic interaction. The Heisenberg model describes the interaction between neighbouring spins and the Hamiltonian for this model, written in terms of the Heisenberg exchange $\mathrm{J}_{i j}$ and the spin operators $\mathrm{S}_{i}$ at sites $i$, has the form

$$
\mathcal{H}=-\sum_{i j} \mathrm{~J}_{i j} \boldsymbol{S}_{i} \cdot \boldsymbol{S}_{j}
$$

where the sum runs over nearest-neighbour pairs at sites $i j$. The spins $\boldsymbol{S}_{i}$ are treated as threedimensional vectors since there is no constraint to them, so they are allowed to point in any direction in the three-dimensional space. While the Hamiltonian above provides a useful starting point for understanding the properties of many geometrically frustrated magnetic materials, for a more realistic model various additional terms to the Hamiltonian $\mathcal{H}$ are required. Perturbations to $\mathcal{H}$ include singleion anisotropy, further neighbour exchange, dipolar interactions, Dzyaloshinskii-Moriya interaction, magnetoelastic coupling, and exchange randomness [6]. We will detail these additional interactions later.

A model in the flair of the previous Hamiltonian is the Ising model, in which the spins of a system can only point up or down. In other words, we restrict to the $z$ component of the spin. The expression for the Hamiltonian of the Ising model is

$$
\mathcal{H}=-\sum_{i j} \mathrm{~J}_{i j} \boldsymbol{S}_{i}^{z} \cdot \boldsymbol{S}_{j}^{z}
$$

here the spins $\boldsymbol{S}_{i}$ are taken as one-dimensional vectors (the spins are only allowed to point along $\pm z)$. These one-dimensional spins can be arranged on a lattice of 1,2 or 3 dimensions [8]. A simple configuration is when the Ising spins are placed on a linear chain. If we assume $\mathrm{J}_{i j}<0$ in the Hamiltonian, we can see that the ground state is obtained by having antiparallel collinear spin 
alignments. Hence the exchange energy is minimized for antiferromagnetic correlations. On the other hand for $\mathrm{J}_{i j}>0$, a parallel spin alignment favours the ground state. Thus the exchange interactions between nearest neighbours (n.n.) are ferromagnetic. Both ordered states are shown in figure 2.1.

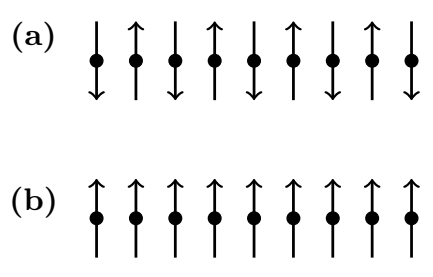

Figure 2.1: Spins on a linear arrangement illustrating its ground state corresponding to antiferromagnetic (a) and ferromagnetic (b) interactions.

We will now study some of the interactions which lead to magnetic ordering in next Subsections. The leading order terms in the magnetic Hamiltonian are the ones which compete directly against each other, thus the additional sub-leading terms in the Hamiltonian will select the ground state in frustrated materials. These can be relatively weak terms which might be ignored in the study of magnetic materials without frustration [6].

\subsubsection{Magnetic dipolar interactions}

Dipolar coupling is the interaction that is the most familiar on a macroscopic scale as it gives rise to observable magnetic fields. When the magnetic moment is small, however, the interaction is weak and is often the contribution of least importance for magnetic order. The energy for the dipolar interaction, for two magnetic spins $\boldsymbol{S}_{i}$ and $\boldsymbol{S}_{j}$, separated by $\boldsymbol{r}_{i j}$ is given by,

$$
\mathcal{H}_{d i p}=\left(g \mu_{\mathrm{B}}\right)^{2} \sum_{i j}\left[\frac{\boldsymbol{S}_{i} \cdot \boldsymbol{S}_{j}}{r_{i j}^{3}}-3 \frac{\left(\boldsymbol{S}_{i} \cdot \boldsymbol{r}_{i j}\right)\left(\boldsymbol{S}_{j} \cdot \boldsymbol{r}_{i j}\right)}{r_{i j}^{5}}\right],
$$

where $g$ is the electron spin $\mathrm{g}$-factor and $\mu_{\mathrm{B}}=9.274 \times 10^{-21} \mathrm{emu}$ is the Bohr magneton. This interaction is generally insignificant as it is much weaker than the exchange interaction but can play an important role in materials that order at millikelvin temperatures with large moments, such as the rare earth pyrochlores. 


\subsubsection{Anisotropic exchange interaction}

Anisotropic exchange occurs when there is an exchange interaction, mediated by spin-orbit coupling, between the excited state of ion $i$ and the excited state of ion $j$. The interaction, known as the Dzyaloshinskii-Moriya interaction, when acting between the spins $\boldsymbol{S}_{i}$ and $\boldsymbol{S}_{j}$ is given by the Hamiltonian:

$$
\mathcal{H}_{D M}=\sum_{i j} \mathrm{~J}_{D M} \hat{d}_{i j} \cdot\left(\boldsymbol{S}_{i} \times \boldsymbol{S}_{j}\right)
$$

here $\mathrm{J}_{D M}$ is the strength of the interaction and $\hat{d}_{i j}$ is the Dzyaloshinskii-Moriya vector. This term is minimized when the $\hat{d}_{i j}$ lies in the direction antiparallel to the products of the spins $\boldsymbol{S}_{i}$ and $\boldsymbol{S}_{j}$. The collective effect of this term causes a small ferromagnetic component to arise perpendicular to the spin-axis of an antiferromagnetically ordered material and to the direction of $\hat{d}_{i j}$.

\subsubsection{Crystal-field interaction}

The crystal field is due to the electric field from neighbouring atoms in the crystal and it depends on the symmetry of the local environment. In a strong crystal field the electrons adopt a low spin configuration and, if it is possible, form pairs in the lowest energy orbitals. On the other hand, for weak crystal field the electrons adopt a high spin configuration occupying all the available orbitals. The interactions between the unpaired spins in the orbitals stabilize a magnetic ordering. For atoms in the $\mathrm{f}$-block, the crystal field lifts the degeneracy of the $2 J+1$ states which minimise Hund's rule (being an exception the case with an exactly half-filled electron shell with zero orbital angular momentum).

\subsubsection{Single-ion anisotropy}

When there is a large crystal field effect the orbital moment may be quenched. The crystalline environment, and the crystal field, tends to be anisotropic and this anisotropy is transferred to the overall spins when there is also large spin-orbit coupling. The effect of the single-ion anisotropy can be large and prevent the spins from aligning with an applied magnetic field. The following term is added to the Hamiltonian to describe this interaction:

$$
\mathcal{H}=D \sum_{i}\left(\boldsymbol{n}_{i} \cdot \boldsymbol{S}_{i}\right)^{2},
$$

where $\boldsymbol{n}_{i}$ is the anisotropy axis and D is a factor that determines the magnitude of the interaction. 


\subsection{Magnetic structures and order}

The long range structure of magnetic materials is dictated by the interactions described in the previous section. The greater the variety of interactions that contribute substantially to the local order, and hence more terms prevail in the Hamiltonian, the more complex is the long range structure that will arise. This section will cover two different types of order: ferromagnetism and antiferromagnetism, which are leading interactions in the geometrically frustrated pyrochlore materials [2].

\subsubsection{Paramagnetism}

In a paramagnetic material, without an applied magnetic field, the magnetic moments point in random directions because the neighbouring moments only interact very weakly with each other and they can be assumed to be independent. Then the magnetization $M$, which is defined as the magnetic moment per unit volume, vanishes for a paramagnet. The application of a magnetic field makes the magnetic moments point out in the direction of the field, the degree of the induced magnetization depends on the strength of the applied magnetic field and on the temperature.

The magnetic moment on an atom is correlated with its total angular moment $J$ which is a sum of the orbital angular momentum $L$ and the spin angular momentum $S$, then

$$
J=L+S
$$

Now we will study quantitatively the magnetization of a spin system in an applied field. Thus, in a field $B$ an atom with angular moment quantum number $J$ has $2 J+1$ energy levels. The magnetization is shown to follow $[9]$

$$
M=n g J \mu_{\mathrm{B}} B_{J}(x),
$$

where $n$ is the spin concentration, $g$ the Landé $\mathrm{g}$-factor, $x=g J \mu_{B} B / k_{B} T$ and $B_{J}$ is the Brillouin function defined as

$$
B_{J}(x)=\frac{2 J+1}{2 J} \operatorname{coth}\left(\frac{2 J+1}{2 J} x\right)-\frac{1}{2 J} \operatorname{coth}\left(\frac{1}{2 J} x\right) .
$$

Then the saturation magnetization $M_{s}$ is the maximum magnetization that can be obtained when all the magnetic moments are aligned. For $x=g J \mu_{\mathrm{B}} B / k_{\mathrm{B}} T \gg 1$ (high magnetic fields and low temperatures) we have $B_{J}(x)=1$, so the magnetization saturation is:

$$
M_{s}=n g J \mu_{\mathrm{B}}
$$


The magnetic susceptibility $\chi$ which is the response of a material to an applied magnetic field is defined by

$$
\chi=\frac{M}{H},
$$

where $M$ is the magnetization and $H$ is the applied magnetic field. For low values of $B$, the magnetic susceptibility, $\chi$, of a paramagnet describes the response to an applied field of an effective moment, $\mathrm{p}_{\text {eff }}$, which is given by $[9]$

$$
\chi=\frac{M}{H} \approx \frac{\mu_{0} M}{B}=\frac{n \mu_{0} \mathrm{p}_{\text {eff }}^{2}}{3 k_{\mathrm{B}} T}=\frac{C}{T} .
$$

Here $\mu_{0}$ is the permeability of free space, $n$ is the number of magnetic moments per unit volume, $k_{\mathrm{B}}=$

$1.38062 \times 10^{-16} \mathrm{erg} . \mathrm{K}^{-1}$ is the Boltzmann constant, $C$ is the Curie constant and $\mathrm{T}$ is the temperature. This dependence of $\chi$ versus $\mathrm{T}$ is called the Curie law, and states that the magnetic susceptibility is inversely proportional to the temperature.

\subsubsection{Ferromagnetism}

A ferromagnet has a spontaneous magnetization even in the absence of an applied field. Here, some of the magnetic moments lie along a single unique direction. This effect is generally due to exchange interactions which were described in the previous section. For a ferromagnet in an applied magnetic field $\boldsymbol{B}$, the appropriate Hamiltonian is:

$$
\mathcal{H}=-\sum_{(i j)} \mathrm{J}_{i j} \boldsymbol{S}_{i} \cdot \boldsymbol{S}_{j}-g \mu_{B} \sum_{(i)} \boldsymbol{S}_{i} \cdot \boldsymbol{B}
$$

and the exchange constants for nearest neighbours will be positive in this case, to ensure ferromagnetic alignment. The first term on the right is the Heisenberg exchange energy. The second term on the right is the Zeeman energy.

\section{The Weiss model of a ferromagnet}

In order to find the transition temperature, it is introduced the Weiss model [8]. This procedure requires to define the effective mean (or molecular) field at the $i$-site given by [8]

$$
\boldsymbol{B}_{m f}=\frac{2}{g \mu_{B}} \sum_{i} \mathrm{~J}_{i j} \boldsymbol{S}_{i}
$$

By focusing on the $i$ th spin in (12), we have that its energy is due to an exchange part and a Zeeman part $-g \mu_{B} \boldsymbol{S}_{i} \cdot \boldsymbol{B}$. The total exchange interaction between the $i$ th spin and its neighbours is 
$-2 \sum_{j} \mathrm{~J}_{i j} \boldsymbol{S}_{i} \cdot \boldsymbol{S}_{j}$, where the factor of 2 is because of the double counting. This term can be written as

$$
-2 \boldsymbol{S}_{i} \sum_{j} \mathrm{~J}_{i j} \cdot \boldsymbol{S}_{j}=-g \mu_{B} \boldsymbol{S}_{i} \cdot \boldsymbol{B}_{m f}
$$

Thus the exchange interaction is replaced by the effective mean field $\boldsymbol{B}_{m f}$ produced by the neighbouring spins. The effective Hamiltonian (12) can now be written as

$$
\mathcal{H}=-g \mu_{B} \sum_{i} \boldsymbol{S}_{i} \cdot\left(\boldsymbol{B}_{m f}+\boldsymbol{B}\right)
$$

which is analogue to the Hamiltonian for a magnet in a magnetic field $\boldsymbol{B}_{m f}+\boldsymbol{B}$. The assumption underlying this approach is that all magnetic ions experience the same mean field. For a ferromagnet the mean field will act so as to align neighbouring magnetic moments. This is because the dominant exchange interactions are positive (for an antiferromagnet, they will be negative).

Since the mean field measures the effect of the ordering of the system, it is assumed that

$$
\boldsymbol{B}_{m f}=\lambda \boldsymbol{M}
$$

being $\lambda$ a constant that parametrizes the strength of the mean field as a function of the magnetization, and for the case of a ferromagnet $\lambda>0$. Then, this problem could be treated as if the system were a simple paramagnet placed in a magnetic field $\boldsymbol{B}_{m f}+\boldsymbol{B}$. We will detail this later when we present the Curie-Weiss law for the magnetic susceptibility. At low temperatures, the moments can be aligned by the internal mean field, even without any applied field being present. As the temperature is raised, thermal fluctuations begin to progressively destroy the magnetization and at a critical temperature the order will be destroyed.

In ferromagnets, below the Curie temperature $\mathrm{T}_{\mathrm{C}}$ the magnet orders spontaneously and gives rise to bulk magnetism. The Curie temperature is the point at which the susceptibility presents an anomaly, and is given by $[8]$

$$
\mathrm{T}_{\mathrm{C}}=\frac{g \mu_{\mathrm{B}}(J+1) \lambda M_{s}}{3 k_{\mathrm{B}}}=\frac{n \lambda \mathrm{p}_{e f f}^{2}}{3 k_{\mathrm{B}}} .
$$

Here $\lambda$ is the parameter representing the mean field, $M_{s}$ is the saturation magnetization, $\mathrm{p}_{e f f}=$ $g \mu_{B} \sqrt{J(J+1)}$ the effective magnetic moment, $J$ total quantum number, $g$ Landé $\mathrm{g}$-factor, $\mu_{\mathrm{B}}=$ $9.274 \times 10^{-21} \mathrm{emu}$ the Bohr magneton and $k_{\mathrm{B}}=1.38062 \times 10^{-16}$ erg.K ${ }^{-1}$ Boltzmann's constant. From equations (12), (15), and (16), the order parameter $\lambda$ is related to the exchange constant $\mathrm{J}$ by [8]:

$$
\lambda=\frac{2 z \mathrm{~J}}{n g^{2} \mu_{\mathrm{B}}^{2}},
$$


where $z$ is the number of nearest neighbours of an ion. Finally, by substituting $\lambda$ in (17) we obtain the Curie temperature $\mathrm{T}_{\mathrm{C}}$ also in terms of the exchange constant $\mathrm{J}$ :

$$
\mathrm{T}_{\mathrm{C}}=\frac{2 z \mathrm{~J}}{3 k_{\mathrm{B}}} J(J+1) .
$$

\subsubsection{Antiferromagnetism}

On antiferromagnets, the exchange interaction is $\mathrm{J}<0$ and because of the mean field it is favourable for nearest neighbour magnetic moments to adopt an antiparallel configuration. Antiferromagnetic order can be considered as two interpenetrating sublattices in which one sublattice has the magnetic moments pointing up and the other pointing down as shown in figure 2.2 from [8].
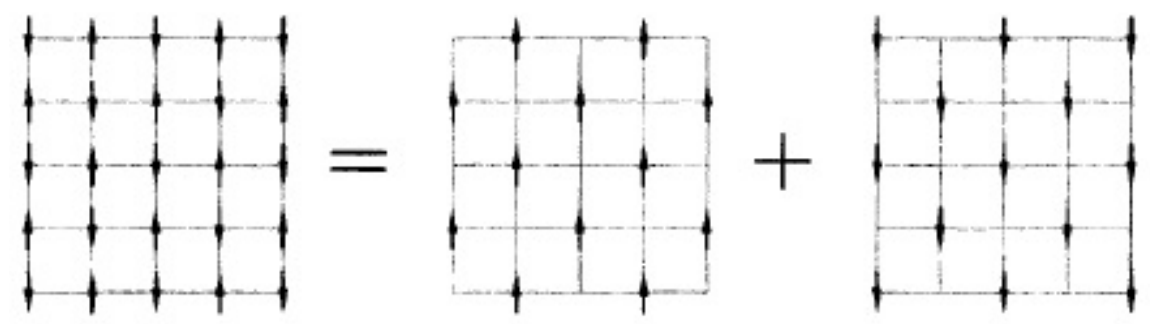

Figure 2.2: An antiferromagnet can be considered as two interpenetrating sublattices.

\section{The Weiss model of an antiferromagnet}

Considering a simple antiferromagnet composed of a positive, + , and negative, - , sublattice the mean field on each sublattice is:

$$
\boldsymbol{B}_{+}=-|\lambda| \boldsymbol{M}_{-} \text {and } \boldsymbol{B}_{-}=-|\lambda| \boldsymbol{M}_{+},
$$

which gives the relation for the transition temperature known as the Neél temperature $T_{N}[8]$ :

$$
\mathrm{T}_{\mathrm{N}}=\frac{g \mu_{B}(J+1)|\lambda| M_{s}}{3 k_{B}}=\frac{n|\lambda| p_{e f f}^{2}}{3 k_{B}} .
$$

This relation is similar to the Curie temperature $T_{C}$, but now the constant $\lambda$ is negative because of the negative exchange interaction J. For an antiferromagnet, $T_{N}$ depends on the exchange constant $J$ as equation (19) of a ferromagnet. Thus, according to [8]

$$
\mathrm{T}_{\mathrm{N}}=\frac{2 z \mathrm{~J}}{3 k_{\mathrm{B}}} J(J+1) .
$$




\subsubsection{Magnetic susceptibility}

In the paramagnetic phase an applied field $\boldsymbol{B}$ will originate a finite magnetization $\boldsymbol{M}$ and consequently a mean field $\boldsymbol{B}_{m f}$. Being $\chi$ the paramagnetic susceptibility, the magnetization and the total magnetic field are related by [9]

$$
\boldsymbol{M}=\chi\left(\boldsymbol{B}+\boldsymbol{B}_{m f}\right) .
$$

The paramagnetic susceptibility $\chi$ is given by the Curie law $\chi=C / T$, where $C$ is the Curie constant. From (16) and (23): $\boldsymbol{M}=C / T(\boldsymbol{B}+\lambda \boldsymbol{M})$. So, the susceptibility now is given by [9]:

$$
\chi=\frac{M}{B}=\frac{C}{T-C \lambda},
$$

Thus, the magnetic susceptibility as a function of the temperature is described by the Curie-Weiss law. It states that [8]:

$$
\chi=\frac{C}{T-\theta_{\mathrm{CW}}},
$$

where $\theta_{\mathrm{CW}}$ is the Curie-Weiss temperature and the Curie constant is $C=n \mathrm{p}_{e f f}^{2} / 3 k_{B}$ with $n$ the spin concentration, $\mathrm{p}_{\mathrm{eff}}=g \mu_{\mathrm{B}} \sqrt{J(J+1)}$ the effective magnetic moment, and $J$ the total quantum number. This result comprises the magnetic susceptibility for the paramagnet, ferromagnet, and antiferromagnet in the mean field approximation. Thus, if the material is a paramagnet, $\theta_{\mathrm{CW}}=0$. For a ferromagnet, $\theta_{\mathrm{CW}}>0$ and it is expected that $\theta_{\mathrm{CW}}=\mathrm{T}_{\mathrm{C}}$. For an antiferromagnet, $\theta_{\mathrm{CW}}<$ 0 and $\theta_{\mathrm{CW}}=-\mathrm{T}_{\mathrm{N}}$. From the above, $\theta_{\mathrm{CW}}$ is equivalent to (19) for a ferromagnet or to (22) for an antiferromagnet. The behaviour of the inverse of susceptibility $1 / \chi$ versus temperature is shown in figure 2.3.

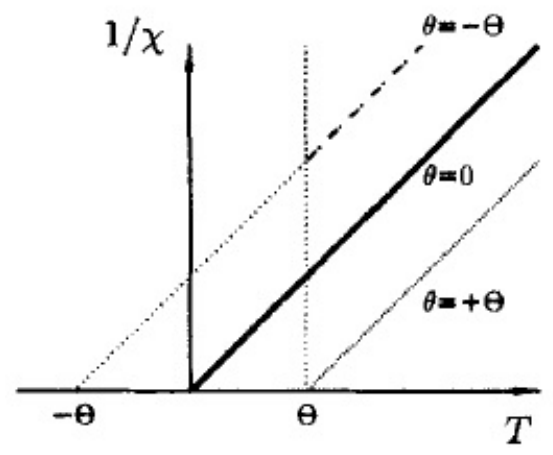

Figure 2.3: Inverse of magnetic susceptibility against temperature graphs for $\theta_{\mathrm{CW}}=0$ (paramagnet), $\theta_{\mathrm{CW}}=\Theta>0$ (ferromagnet) and $\theta_{\mathrm{CW}}=\Theta<0$ (antiferromagnet). This figure was taken from [8]. 
From (25) the inverse magnetic susceptibility is:

$$
\chi^{-1}=\frac{T}{C}-\frac{\theta_{C W}}{C}
$$

and

$$
\frac{\theta_{C W}}{C}=\frac{3 k_{\mathrm{B}} \theta_{\mathrm{CW}}}{n g^{2} \mu_{\mathrm{B}}^{2} J(J+1)}
$$

\subsubsection{Specific heat}

The specific heat at constant volume $C_{v}$ of a solid can be calculated from its internal energy $U$ and the temperature $T$ as

$$
C_{v}=\left(\frac{\partial U}{\partial T}\right)_{v}
$$

For purposes of actual measurement, the specific heat at constant pressure, $C_{p}$, is much more convenient, but actually in a solid the difference $\left(C_{p}-C_{v}\right)$ is almost negligible. Furthermore, what it is measured experimentally at any temperature $\mathrm{T}$ is the total specific heat $C$ of the solid. Then, we can separate the different contributions to $C$ as

$$
C=C_{l a t}+C_{e l}+C_{m}+C_{N}
$$

where $C_{l a t}$ is the specific heat due to the phonons, $C_{e l}$ is the contribution of the conduction electrons, $C_{m}$ is the magnetic contribution, and $C_{N}$ is the nuclear contribution.

Firstly, we will study the case of the phonons or lattice contribution to the specific heat. The Debye model provides an excellent fit to the low temperature specific heat of the phonons of many solids. The procedure supposes that the modes of vibration at frequencies $\omega$ of the phonons are subject to a density of states $g(\omega)$ and that the phonon occupancy is given by the Bose-Einstein statistics [10]. Then, the thermal energy $U$ is $[10]$

$$
U=\int \frac{(\hbar \omega) \cdot g(\omega) \cdot d \omega}{\left[\exp \left(\hbar \omega / k_{\mathrm{B}} T\right)-1\right]}
$$

In the Debye model the speed of sound $v_{0}$ is taken as constant for each polarization type, as in a classical elastic continuum, then $g(\omega)=3 \omega^{2} / 2 \pi^{2} v_{0}^{3}$ [10]. In addition, a solid comprising $N$ atoms in a volume $V$ must have $(3 N / V)$ modes per unit volume which sets a cutoff frequency $\omega_{D}[10]$. The resulting lattice vibrational energy per unit volume is then [10]

$$
U=9 n k_{\mathrm{B}} T\left(\frac{T}{\Theta_{D}}\right)^{3} \int_{0}^{\Theta_{D} / T} \frac{x^{3} d x}{\left(e^{x}-1\right)}
$$


where $x=\hbar \omega / k_{\mathrm{B}} T$ is a dimensionless substitution, $\Theta_{D}=\hbar \omega_{D} / k_{\mathrm{B}}=\left(\hbar v_{0} / k_{\mathrm{B}}\right)\left(6 \pi^{2} N / V\right)^{1 / 3}$ is the Debye characteristic temperature, and $n=N / V$ is the atom concentration in the solid. Differentiating (31) with respect to the temperature, the specific heat is [10]

$$
C_{l a t}=9 n k_{\mathrm{B}}\left(\frac{T}{\Theta_{D}}\right)^{3} \int_{0}^{\Theta_{D} / T} \frac{x^{4} e^{x} d x}{\left(e^{x}-1\right)^{2}} .
$$

In the low-temperature regime, that is, for $x=\hbar \omega / k_{\mathrm{B}} T \gg 1$, the lattice contribution to the specific heat is given by $[10]$

$$
C_{\text {lat }}=\frac{12 \pi^{4}}{5} n k_{\mathrm{B}}\left(\frac{T}{\Theta_{D}}\right)^{3}=\beta T^{3} .
$$

Secondly, we will discuss the case of the electronic contribution to the specific heat. Here, the total energy $U$ of the conduction electrons which obey the Fermi-Dirac statistics can be written as [9]

$$
U=\int \epsilon \cdot g(\epsilon) \cdot f(\epsilon) d \epsilon
$$

where $\epsilon=\hbar^{2} k^{2} / 2 m$ is the electronic energy for a electron gas, $g(\epsilon)=\left(m / \pi^{2} \hbar^{2}\right)\left(2 m \epsilon / \hbar^{2}\right)^{1 / 2}$ is the density of electron states, and $f(\epsilon)$ is the Fermi-Dirac distribution. In the ground state of a system of $\mathrm{N}$ free electrons in a volume $\mathrm{V}$ the occupied orbitals can be represented as points inside a sphere in $\boldsymbol{k}$ space [9]. The highest energy of the electrons corresponds to the surface of a sphere with radius $k_{F}$ and is known as the Fermy energy $\epsilon_{F}=\hbar^{2} k_{F}^{2} / 2 m$. Then, the total energy $U$ is [9]

$$
U=\frac{\pi^{2}}{6}\left(k_{\mathrm{B}} T\right)^{2} g\left(\epsilon_{F}\right)
$$

where the density of levels at the Fermy energy is $g\left(\epsilon_{F}\right)=\left(3 n_{e} / 2 \epsilon_{F}\right)$ with $n_{e}=N_{e} / V$ the electronic density and $\epsilon_{F}$ is the Fermi energy which can be written as [9]

$$
\epsilon_{F}=\frac{\hbar^{2}}{2 m}\left(3 \pi^{2} n_{e}\right)^{2 / 3}
$$

From (35), the specific heat of the electrons at low temperatures $k_{B} T \ll \epsilon_{F}$ is therefore [9]

$$
C_{e l}=\frac{\pi^{2}}{3} k_{\mathrm{B}}^{2} T g\left(\epsilon_{F}\right)
$$

or in terms of the Fermy energy

$$
C_{e l}=\frac{\pi^{2}}{2}\left(\frac{k_{\mathrm{B}} T}{\epsilon_{F}}\right) n_{e} k_{\mathrm{B}}=\gamma T .
$$

Thirdly, we will study the case of the magnetic contribution $\mathrm{C}_{m}$ to the specific heat. As we will discuss later, in the pyrochlore $\mathrm{Gd}_{2} \mathrm{Ti}_{2} \mathrm{O}_{7}$ the leading interaction is the antiferromagnetic Heisenberg 
exchange, then the magnetic specific heat $\mathrm{C}_{m}$ is computed by considering magnons or spin waves, which are collective spin excitations, in an antiferromagnet. The procedure proposed in [11] for another pyrochlore antiferromagnet $\mathrm{Er}_{2} \mathrm{Ti}_{2} \mathrm{O}_{7}$ assumes that the dispersion relation $\hbar \omega(\boldsymbol{q})$ for their lowest energy can be approximated at small wave vectors as

$$
\hbar^{2} \omega^{2}(\boldsymbol{q})=\hbar^{2} \omega^{2}(q)=\triangle^{2}+\hbar^{2} v_{s w}^{2} q^{2}
$$

where $\triangle$ is the gap energy of the spectrum at the zone center and $v_{s w}$ is the magnon velocity. When $\triangle$ is negligible, the magnon specific heat is calculated in the temperature regime where small wave vectors occur that is when (39) applies. According to [11], the $T^{3}$ law for the specific heat $C_{m}$ in a pyrochlore antiferromagnet is

$$
C_{m}=\frac{\pi^{2}}{120} N_{A} \frac{k_{\mathrm{B}}^{4} a^{3}}{\hbar^{3} v_{s w}^{3}} T^{3}=\Lambda T^{3},
$$

where $N_{A}$ is Avogadro's constant and $a$ the lattice parameter. Later, from our specific heat measurements we will discuss the magnon velocity in different pyrochlores. Then, by replacing the values of the constants in (40), the magnon velocity is given by

$$
v_{s w}=\left(1537 \cdot \frac{a^{3}}{\Lambda}\right)^{1 / 3} .
$$

Here $a$ in $\AA$ units and $\Lambda$ in $\mathrm{J} . \mathrm{K}^{-4} \cdot \mathrm{mol}^{-1}$ return a value in $\mathrm{m} / \mathrm{s}$ for $v_{s w}$.

Finally, we will discuss the case of the nuclear contribution $C_{N}$ to the specific heat. This contribution arises from the combination of a nuclear electric quadrupole interaction and a nuclear magnetic hyperfine interaction of the magnetic atoms. Then, the nuclear specific heat is given by [12]

$$
C_{N}=\frac{R}{\left(k_{\mathrm{B}} T\right)^{2}} \frac{\sum_{i, j}\left(W_{i}^{2}-W_{i} W_{j}\right) \exp \left[-\left(W_{i}+W_{j}\right) / k_{\mathrm{B}} T\right]}{\sum_{i, j} \exp \left[-\left(W_{i}+W_{j}\right) / k_{\mathrm{B}} T\right]},
$$

where $\mathrm{i}, \mathrm{j}=-\mathrm{I},-\mathrm{I}+1, \ldots, \mathrm{I}$ with $\mathrm{I}$ the number of spin nuclear, and $W_{i}$ the potential energy of the $i$ level.

The pyrochlore material discussed in this dissertation: $\mathrm{Gd}_{2} \mathrm{Ti}_{2} \mathrm{O}_{7}$ is an insulator $(\gamma=0)$ so the electronic contribution will not be considered to the total specific heat $C$. Nuclear contributions to the specific heat are also neglected because in $\mathrm{Gd}_{2} \mathrm{Ti}_{2} \mathrm{O}_{7}$ they arise at temperatures of the order of ten $\mathrm{mK}$, where we did not perform thermal measurements. Then, the total specific heat is considered as

$$
C=C_{\text {lat }}+C_{m}=\beta T^{3}+\Lambda T^{3} .
$$


An additional contribution to the specific heat that we will study is known as the Schottky anomaly. This anomaly occurs in systems with quantized energy levels and is reflected in the specific heat by the presence of a maximum. For example, for a spin $S$ there are $2 S+1$ possible orientations of the spin; in a magnetic field, there are a number of discrete energy levels. So, when the temperature is comparable to the energy separation there is a broad peak in the specific heat due to a large change in entropy for a small change in temperature. For a system with two energy levels the Schottky specific heat $C_{\mathrm{Sch}}$ at a temperature $T$ is given by [13]

$$
C_{\mathrm{Sch}}=R\left(\frac{\delta}{T}\right)^{2} \frac{g_{0}}{g_{1}} \frac{\exp (\delta / T)}{\left[1+\left(g_{0} / g_{1}\right) \exp (\delta / T)\right]^{2}},
$$

where $g_{0}$ and $g_{1}$ are the degeneracies of the two energy levels, $\delta=\triangle E / k_{\mathrm{B}}$ is the energy separation in Kelvin units, and $R=8.314 \mathrm{~J} . \mathrm{K}^{-1} \cdot \mathrm{mol}^{-1}$ is the gas constant. For spins with magnetic moment $\mu$ in an external magnetic field $B$, the energy separation is $\triangle E=2 \mu B$. 


\subsection{Geometric frustration}

As mentioned earlier in the Introduction of this dissertation, the phenomenon of frustration is present in systems with competing interactions among the components. Thus, frustration is defined as a system's inability to simultaneously minimize the competing interaction energies between all its components [1], or in simpler terms that the system cannot minimize its energy or find a unique ground state. In addition to that, frustration occurs as a result of each interaction in the system opting for favoring its own characteristic spatial correlations [2]. Within the context of competing interactions is the topic of geometric frustration, which is the core for exotic magnetic phenomena present in the pyrochlore materials and that we will discuss later. When frustration is determined purely by the geometry (topology) of the lattice it is termed geometric frustration. That means that the system cannot minimize its energy because of the spatial arrangement of the components.

The fundamentals underlying geometric frustration are easy to understand in the frame of magnetic materials. In many magnetic spin systems, nearest-neighbour Heisenberg exchange interaction dominates. The examples in one dimension of the previous subsection illustrate that, for simple lattices, minimising the nearest-neighbour exchange interaction (antiferromagnetic or ferromagnetic) specifies a unique ground state. On the other hand, novel exotic ground states arises when those exchange interactions compete in more complex lattices. Geometric frustration in a magnetic system arises when the competing interactions between spins are subject to constraints imposed by the geometry which cannot be satisfied by simple co-linear orderings [7]. The inability of frustrated systems to satisfy all pairs of interactions is reflected by the fact that a large number of different magnetic configurations minimize the classical energy, hence leading to an extensive degeneracy [14]. Thus, the study of geometrically frustrated magnetic systems is concerned with what happens when lattice geometry inhibits the formation of a simple, ordered, and low-temperature spin configuration.

The canonical example of a geometrically frustrated lattice is the equilateral triangle in which the spins lie at the vertices as shown in figure 2.4. For Ising spins, which can only point up or down, interacting with the simple Hamiltonian $\mathcal{H}=-\sum \mathrm{J}_{i j} \boldsymbol{S}_{i} \cdot \boldsymbol{S}_{j}$ via nearest-neighbour antiferromagnetic exchange $\left(\mathrm{J}_{i j}<0\right)$ we see that it is impossible for the three spins to satisfy each bond simultaneously. This is because once two of the spins are antialigned to satisfy their antiferromagnetic interaction, the third one can no longer point in a direction opposite to both other spins. Also it is important to notice that the lowest energy state of these three spins is not unique, but six equal energy states exist. When many triangles are condensed to form an edge-sharing triangular lattice the frustration of the system 
increases massively. The triangular lattice is an example of geometric frustration because it is the regular periodic structure of the space lattice that imposes the constraint inhibiting the development of a long-range ordered state given antiferromagnetic interactions between the spins [2]. If we consider the tetrahedron as shown in figure 2.4 instead of a singular triangular lattice it can be seen that if the bondings are antiferromagnetic the same situation arises as all the pairwise interactions cannot be simultaneously satisfied.
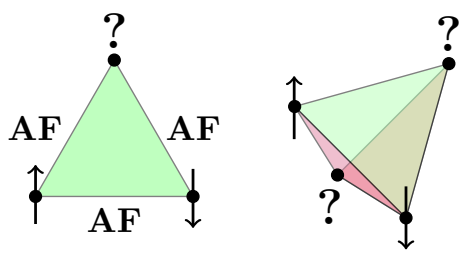

Figure 2.4: Spins with antiferromagnetic exchange arranged on a triangle or tetrahedron are geometrically frustrated. In the triangle one-third of the sites are always frustrated, while for the tetrahedron two of the four sites.

For the case of Heisenberg spins, we illustrate geometric frustration on a system of spins which lie at the vertices of two corner-sharing triangles with nearest-neighbour antiferromagnetic interactions. Here, the ground states are configurations in which spins within each triangle are coplanar and at relative angles of $2 \pi / 3$. There is also a degeneracy arising from rotations about the common spin, as indicated in figure 2.5 .

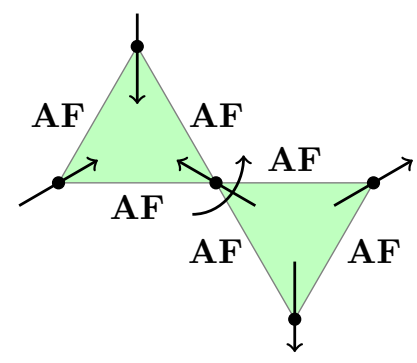

Figure 2.5: Heisenberg spins in a triangle lattice are subject to frustration.

But why do the geometrically frustrated magnets have such importance in condensed matter physics? The first reason is that geometric frustration inhibits the formation of long range ordered spin ground states, that means the system is impeded from removing its spin entropy because of the extensive degeneracy of the ground state, as is demanded by the third law of thermodynamics. This 
results in exotic spin dynamics, which can be studied by different means, such as magnetization, ac susceptibility, Mössbauer spectroscopy and neutron scattering [7]. The second one is that due to the large degeneracy of the ground state, it results in a macroscopic problem with a degeneracy of the order of the number of magnetic sites, that is, $\sim 10^{22}[7]$. This large degeneracy gives rise to complex long range order or exotic short range ordered ground states as in spin liquids or spin ices [7].

Figure 2.6 shows some examples of geometrically frustrated lattices that are based on the canonical equilateral triangle or tetrahedron, which include the edge shared triangular lattice (a), the corner shared triangular lattice known as Kagomé (b), the edge shared tetrahedral lattice or face centered cubic lattice (c) and the corner sharing tetrahedral lattice or pyrochlore (d). The pyrochlore lattice occurs in spinel materials and $\mathrm{A}_{2} \mathrm{~B}_{2} \mathrm{O}_{7}$ compounds. The latter will be described in detail in the next section.

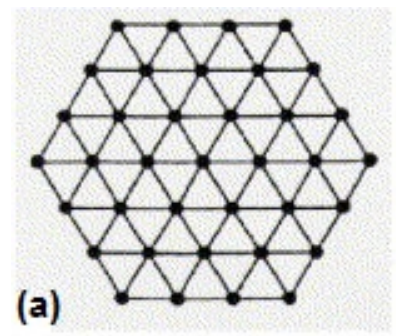

(b)
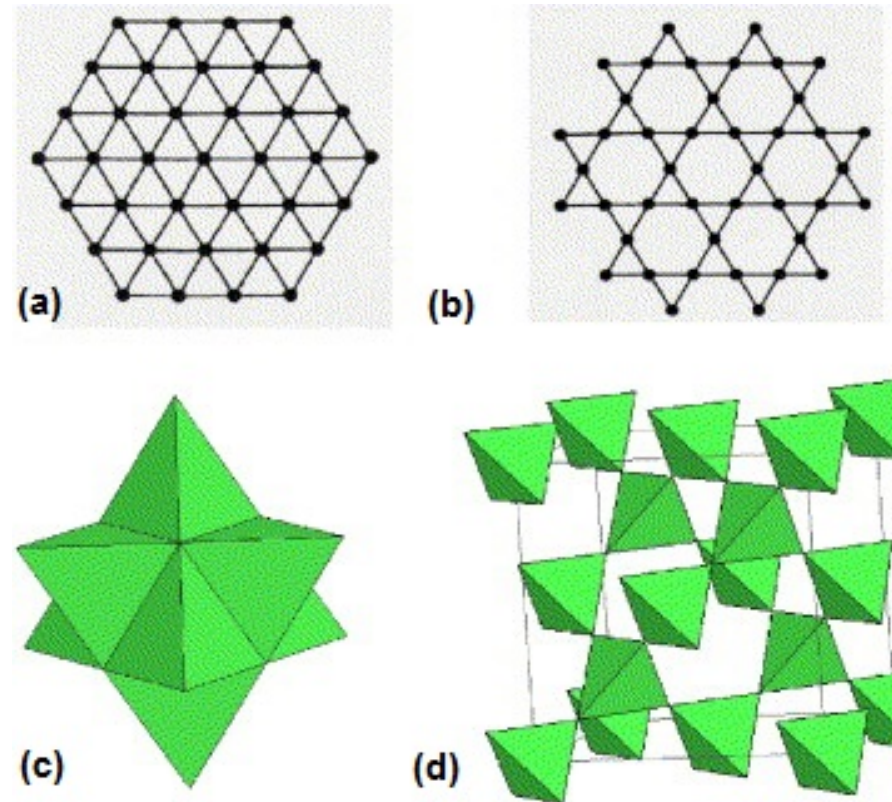

(d)

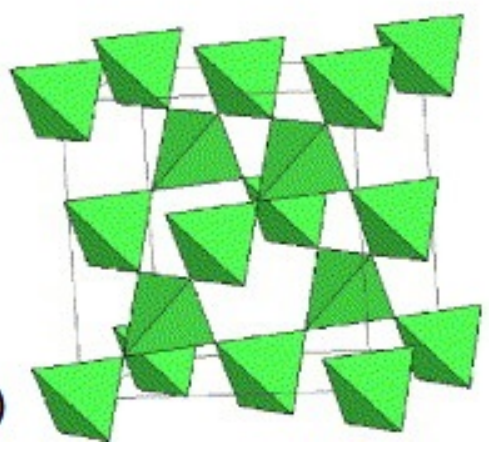

Figure 2.6: Geometrically frustrated lattices: (a) edge-sharing triangular, (b) corner-sharing triangular or Kagomé, (c) edge-sharing tetrahedral and (d) corner-sharing tetrahedral or pyrochlore. Figure taken from [7].

In the laboratory, how do we identify geometrically frustrated materials? We identify them because their magnetic susceptibility has a characteristic behaviour. The inverse susceptibility, $\chi^{-1}$, follows the usual Curie-Weiss law down to temperatures well below the expected mean-field-ordering transition 
temperature $\theta_{\mathrm{CW}}$. At some low temperature $\mathrm{T} \ll \theta_{\mathrm{CW}}$ substantial deviations from the linear behaviour occur, typically signaling a transition to a state that differs from compound to compound, which may, for example, be ordered or glassy [6]. Figure 2.7 displays how for a frustrated antiferromagnet the behaviour of the inverse susceptibility differs from the antiferromagnet or ferromagnet.

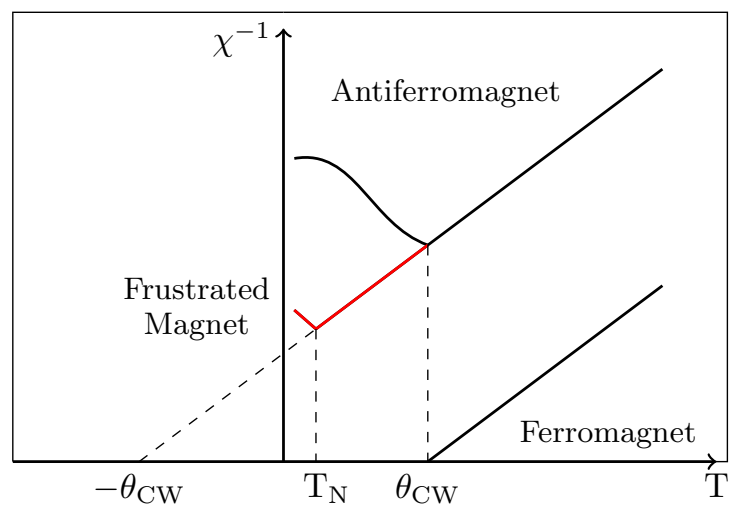

Figure 2.7: Characteristic behaviour $\chi^{-1}$ vs $\mathrm{T}$ of a geometrically frustrated antiferromagnet (red line). The transition occurs at the temperature $T_{\mathrm{N}} \ll \theta_{\mathrm{CW}}$.

In order to measure the level of frustration of our magnetic systems, we calculate the ratio between the Curie-Weiss temperature $\theta_{\mathrm{CW}}$ and the transition temperature $\mathrm{T}^{*}$ which defines the frustration index $f[2,15]$ :

$$
f \equiv \frac{\left|\theta_{\mathrm{CW}}\right|}{\mathrm{T}^{*}} .
$$

Here $\theta_{\mathrm{CW}}$ is defined from the high-temperature paramagnetic response of the system that is from the linear Curie-Weiss behaviour of the inverse susceptibility. The temperature $\mathrm{T}^{*}$ is the transition temperature or Néel temperature $\mathrm{T}_{\mathrm{N}}$ at which the system develops long-range spin order [2]. Thus, large values of $f$ are a signature of frustration [16]. This frustration index will be calculated recurrently in Chapters 4 and 5 for the pyrochlore $\mathrm{Gd}_{2} \mathrm{Ti}_{2} \mathrm{O}_{7}$ with lattice defects such as oxygen vacancies and yttrium dilution in order to compare the degree of frustration of similar samples. 


\subsection{Rare Earth Pyrochlores}

In this section we will study the rare earth pyrochlores in detail and also we will review briefly the novel microscopic behaviours of some of these materials. First, we will describe the crystalline structure of rare earth pyrochlores which, as mentioned before, have the general formula $\mathrm{A}_{2} \mathrm{~B}_{2} \mathrm{O}_{7}$. Next, we will focus on the compounds of the $\mathrm{B}=$ Ti series which suffer phase transitions to novel magnetic ground states reported on previous works $[2,7]$. Then, we will study some of those magnetic phases that the Ti series of the rare earth pyrochlores present, such as the long-range ordered, the spin-ice, and the spin-liquid phase. Finally, examples of pyrochlores for each of these exotic magnetic phases will be introduced.

\subsubsection{Pyrochlore structure}

The pyrochlore materials have a general formula $\mathrm{A}_{2} \mathrm{~B}_{2} \mathrm{O}_{7}$, where $\mathrm{A}$ is a trivalent rare earth, $\mathrm{A}^{3+}$, which includes the lanthanides (Gd, Tb, Dy, Ho, Er, Yb) and yttrium, and B is either a transition metal or a p-block metal ion (Ti, Sn, Mn, Mo, $\mathrm{Pb}$ ) with valence $\mathrm{B}^{4+}$. Pyrochlore materials $\mathrm{A}_{2} \mathrm{~B}_{2} \mathrm{O}_{7}$ are oxides that crystallize in the space group $F d \overline{3} m$. The standard method to study the crystal structure of pyrochlores is to formulate them as $\mathrm{A}_{2} \mathrm{~B}_{2} \mathrm{O}_{6} \mathrm{O}^{\prime}$ and to place the $\mathrm{B}$ ion at $16 c, \mathrm{~A}$ at $16 d$, $\mathrm{O}$ at $48 f$ and $\mathrm{O}^{\prime}$ at $8 b[2]$. This is shown in table 2.1 where $\mathrm{O}^{\prime}$ and $\mathrm{O}$ are labeled as $\mathrm{O}(1)$ and $\mathrm{O}(2)$ respectively. There is only one adjustable positional parameter $x$ for the $\mathrm{O}(2)$ atom at the site $48 f$.

\begin{tabular}{c|c|c|c}
\hline Atom & Wyckoff positions & Point symmetry & Minimal coordinates \\
\hline \hline $\mathrm{A}$ & $16 d$ & $\overline{3} m\left(D_{3 d}\right)$ & $1 / 2,1 / 2,1 / 2$ \\
\hline $\mathrm{B}$ & $16 c$ & $\overline{3} m\left(D_{3 d}\right)$ & $0,0,0$ \\
\hline $\mathrm{O}(1)$ & $8 b$ & $\overline{4} 3 m\left(T_{d}\right)$ & $3 / 8,3 / 8,3 / 8$ \\
\hline $\mathrm{O}(2)$ & $48 f$ & $m m\left(C_{2 v}\right)$ & $\mathrm{x}, 1 / 8,1 / 8$ \\
\hline \hline
\end{tabular}

Table 2.1: The crystallographic positions for the space group $F d \overline{3} m$ suitable for the cubic pyrochlore $\mathrm{A}_{2} \mathrm{~B}_{2} \mathrm{O}_{6} \mathrm{O}^{\prime}$ with origin at $16 c$.

Both the A site and the B site, independently, reside on a network of corner-sharing tetrahedra giving rise to the geometrically frustrated pyrochlore lattice - as shown in figure 2.8. Also, the two pyrochlore lattices of the cations $\mathrm{A}^{3+}$ and $\mathrm{B}^{4+}$ are displaced from each other by a translation along the cubic diagonal [111]. From figure 2.8, there are two types of tetrahedra in the pyrochlores, which 
are labeled as the down tetrahedra and the up tetrahedra. Each down tetrahedron is connected to four up ones, and vice versa. The cube represents a unit cell with eight tetrahedra (four of each kind) and 16 cations or spins. Thus a unit cell contains 8 chemical units $\mathrm{A}_{2} \mathrm{~B}_{2} \mathrm{O}_{7}$. For the rare earth pyrochlores the length of a unit cell is $a \sim 10 \AA$ hence the distance between nearest neighbours is $r_{\mathrm{nn}}=\frac{\sqrt{2}}{4} a \sim$ $3.5 \AA$ and the distance between the centres of two tetrahedra is $r_{\mathrm{d}}=\frac{\sqrt{3}}{4} a \sim 4.3 \AA$ [17]. The smallest closed loop in the pyrochlore lattice comprises six spins (green dotted loop).

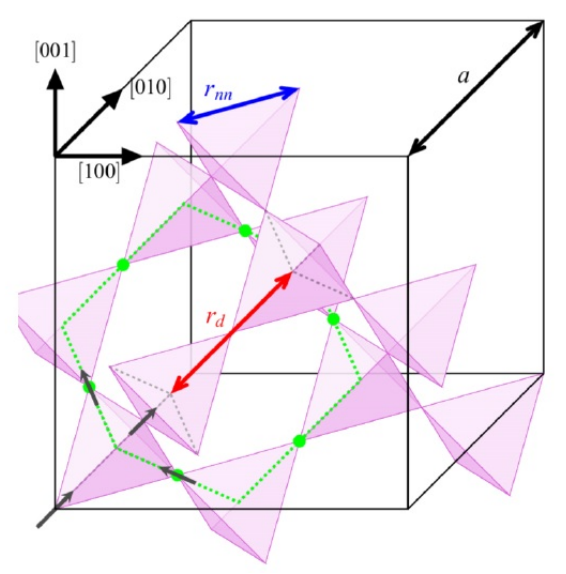

Figure 2.8: Pyrochlore lattice: the rare-earth ions $\mathrm{A}^{3+}$ of $\mathrm{A}_{2} \mathrm{~B}_{2} \mathrm{O}_{7}$ are located on the vertices of every tetrahedron. The ions $\mathrm{B}^{4+}$ sit on an identical lattice displaced by a translation along the cubic diagonal [111]. Figure taken from [17].

Having defined the atomic positions on the lattice and the array of corner-sharing tetrahedra for pyrochlores $\mathrm{A}_{2} \mathrm{~B}_{2} \mathrm{O}_{7}$ in general, we now restrict to the pyrochlore materials belonging to the titanate series. These compounds with the rare earth ion $\mathrm{A}^{3+}$ and $\mathrm{B}$ being $\mathrm{Ti}^{4+}$ are of formula $\mathrm{A}_{2} \mathrm{Ti}_{2} \mathrm{O}_{7}$. Rare earth pyrochlores in the titanate series display a wide range of magnetic phenomena when they are studied at very low temperatures. We will study in next Subsection some of the magnetic ground states that the pyrochlores $\mathrm{A}_{2} \mathrm{Ti}_{2} \mathrm{O}_{7}$ present, such as spin ice, spin-liquids, and long-range order. Remarkable examples of spin ice are $\mathrm{Ho}_{2} \mathrm{Ti}_{2} \mathrm{O}_{7}$ and $\mathrm{Dy}_{2} \mathrm{Ti}_{2} \mathrm{O}_{7}$; of spin liquid is $\mathrm{Tb}_{2} \mathrm{Ti}_{2} \mathrm{O}_{7}$; and exhibiting long-range order are $\mathrm{Gd}_{2} \mathrm{Ti}_{2} \mathrm{O}_{7}$ and $\mathrm{Gd}_{2} \mathrm{Sn}_{2} \mathrm{O}_{7}[2]$. 


\subsubsection{Ordered phases in pyrochlore oxides}

The pyrochlore oxides mentioned before enter in the magnetic phases of spin ice, spin liquids, and long-range order as we will discuss here. Since the central theme of this dissertation is the pyrochlore $\mathrm{Gd}_{2} \mathrm{Ti}_{2} \mathrm{O}_{7}$ which is subject to long-range order [18], we will discuss briefly the spin-ice and spinliquid phases. However, we will emphasize the magnetic properties of spin ices because we will go back to them in Chapter 4. First, we will begin by introducing the spin-ice phase in $\mathrm{Ho}_{2} \mathrm{Ti}_{2} \mathrm{O}_{7}$ and $\mathrm{Dy}_{2} \mathrm{Ti}_{2} \mathrm{O}_{7}$, and how its analogy with the problem of water ice led to determine its ground state. Next, we will explain in general words the main properties involving spin liquids and also their experimental realizations $\mathrm{Yb}_{2} \mathrm{Ti}_{2} \mathrm{O}_{7}$ and $\mathrm{Tb}_{2} \mathrm{Ti}_{2} \mathrm{O}_{7}$. Finally, we will move on to consider the long-range ordering in $\mathrm{Gd}_{2} \mathrm{Ti}_{2} \mathrm{O}_{7}$ and $\mathrm{Gd}_{2} \mathrm{Sn}_{2} \mathrm{O}_{7}$.

\section{Spin ice}

Currently the spin-ice phase has been studied extensively in the rare earth pyrochlores $\mathrm{Ho}_{2} \mathrm{Ti}_{2} \mathrm{O}_{7}$ and $\mathrm{Dy}_{2} \mathrm{Ti}_{2} \mathrm{O}_{7}$. The rare-earth ions $\mathrm{Ho}^{3+}$ and $\mathrm{Dy}^{3+}$ present single-ion electronic ground states ${ }^{5} \mathrm{I}_{8}$ and ${ }^{6} \mathrm{H}_{15 / 2}$ respectively. In $\mathrm{Ho}_{2} \mathrm{Ti}_{2} \mathrm{O}_{7}$ the Curie-Weiss temperature is $\theta_{\mathrm{CW}} \sim+2 \mathrm{~K}$, indicating ferromagnetic correlations [19]. However, it was quite unexpected that $\mathrm{Ho}_{2} \mathrm{Ti}_{2} \mathrm{O}_{7}$ does not develop long-range order down to $50 \mathrm{mK}$, as reported by muon spectroscopy and neutron scattering measurements [19]. This presented a paradox, as the dominant spin-spin coupling is apparently ferromagnetic and it was unclear how a ferromagnet could be subject to geometric frustration [7]. As discussed in [20], in $\mathrm{Ho}_{2} \mathrm{Ti}_{2} \mathrm{O}_{7}$ because of the strong crystal field acting on $\mathrm{Ho}^{3+},{ }^{5} \mathrm{I}_{8}$, it stabilises a ground-state doublet of states with $\left|J, m_{j}\right\rangle=|8, \pm 8\rangle$, i.e., gives rise to a classical Ising spin with easy-axis (quantization) along the local $\langle 111\rangle$ direction $[2,7]$. With this constraint on a tetrahedron, a spin can only point "in" toward the centre of the tetrahedron, or point "out" of the tetrahedron. Regarding the problem of the ground state in which the ferromagnetic exchange between spins on the tetrahedron must be minimized, in [20] it was discussed that this ground state or spin configuration could be mapped onto the problem of the proton (hydrogen) positions in water ice studied by Pauling [21]. Thus, the spin configuration minimizing the energy and relieving the geometric frustration is "two spins in, two spins out" called the ground state of spin ice [20]. This is illustrated on the left side of figure 2.9. 

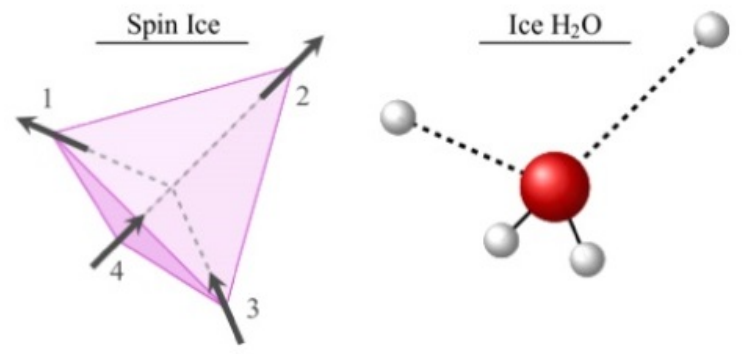

Figure 2.9: Ising spins at each vertices of tetrahedron, oriented along local easy-axis $\langle 111\rangle$, and its analogy with the arrangement of protons (white circles) about oxygen in water ice (red circle). The "spin ice" analogy. Figure taken from [17].

Figure 2.9 from [17] shows how the spins with local $\langle 111\rangle$ direction pointing inside (outside) the tetrahedron correspond to short covalent bonds (long $\mathrm{H}$-bond) for water ice [17]. Pauling showed that for water ice there is a large degeneracy of the ground state, and there is therefore an extensive or residual entropy of the ground state. The value of this residual entropy was calculated by Pauling as $S_{0}=\frac{R}{2} \ln \left(\frac{3}{2}\right)[21]$.

On the other hand, $\mathrm{Dy}_{2} \mathrm{Ti}_{2} \mathrm{O}_{7}$ also presents strongly anisotropic properties as discussed in [22] where the strong Ising nature of $\mathrm{Dy}^{3+}$ with local axis $\langle 111\rangle$ was observed. Similarly to $\mathrm{Ho}^{3+}$ ions, for Ising spins $\mathrm{Dy}^{3+},{ }^{6} \mathrm{H}_{15 / 2}$, the strong crystal field lifts the single-ion electronic states to a doublet with $\left|J, m_{j}\right\rangle=|15 / 2, \pm 15 / 2\rangle$ [23]. The first experimental verification of the spin ice in $\mathrm{Dy}_{2} \mathrm{Ti}_{2} \mathrm{O}_{7}$ was provided by Ramirez et al. in [24]. They observed from the magnetic specific heat $C_{m}(T)$ shown in figure 2.10(a) that the residual entropy in $\mathrm{Dy}_{2} \mathrm{Ti}_{2} \mathrm{O}_{7}$ approached the Pauling value for water ice [24]. The magnetic entropy removed $\delta S_{1,2}$ upon cooling between temperatures $T_{1}$ and $T_{2}$ can be determined from the specific heat measurements using the thermodynamic relationship:

$$
\delta S_{1,2} \equiv \int_{T_{1}}^{T_{2}} \frac{C(T)}{T} d T .
$$

The magnetic entropy $S=k_{\mathrm{B}} \ln (\Omega)$ for a system of $N$ Ising spins (two spin orientations) is determined from its total number of microstates $\Omega=2^{N}$, so the expected entropy is $S=N k_{\mathrm{B}} \ln (2)$. Then, the total magnetic entropy per mol is $R \ln (2)$. Figure 2.10(b) shows that the entropy recovered at $10 \mathrm{~K}$ is $3.9 \mathrm{~J} \cdot \mathrm{K}^{-1} \cdot \mathrm{mol}^{-1}$, a value that is less than the expected $R \ln (2)=5.76 \mathrm{~J} \cdot \mathrm{K}^{-1} \cdot \mathrm{mol}^{-1}$. The difference of entropies, $1.86 \mathrm{~J} . \mathrm{K}^{-1} \cdot \mathrm{mol}^{-1}$, is very close to Pauling's estimate for the residual extensive entropy of water ice, $S_{0}=\frac{R}{2} \ln \left(\frac{3}{2}\right)=1.68 \mathrm{~J} . \mathrm{K}^{-1} \cdot \mathrm{mol}^{-1}$, thus with this result Ramirez et al. proved 
that $\mathrm{Dy}_{2} \mathrm{Ti}_{2} \mathrm{O}_{7}$ carries an extensive entropy close to $S_{0}$ and obeys the ice rules [24].

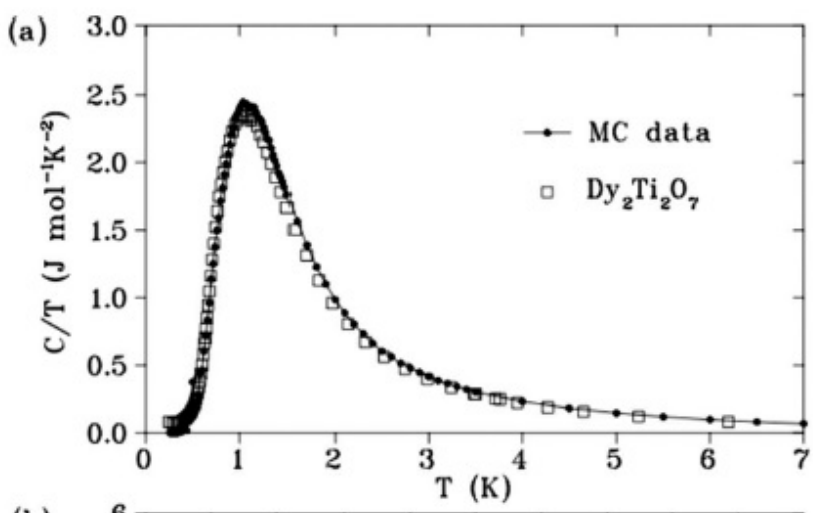

(b)

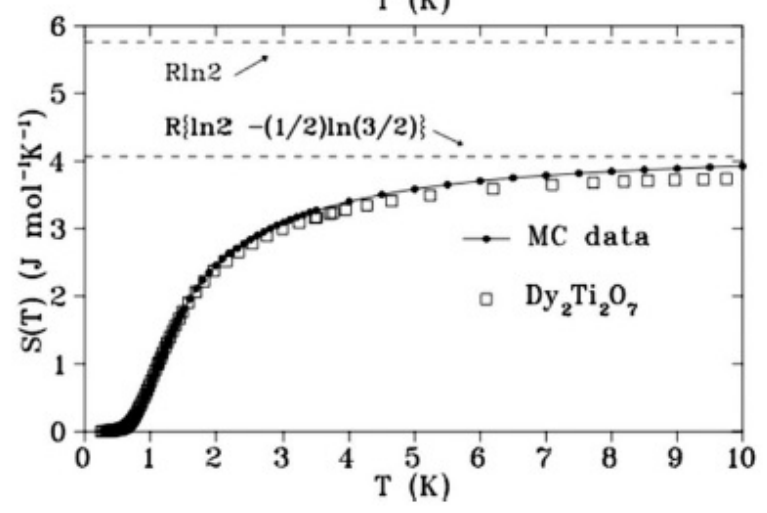

Figure 2.10: $\mathrm{Dy}_{2} \mathrm{Ti}_{2} \mathrm{O}_{7}$ : (a) Specific heat and (b) entropy as a function of the temperature reported by [24]. The residual entropy of $\mathrm{Dy}_{2} \mathrm{Ti}_{2} \mathrm{O}_{7}, 1.86 \mathrm{~J} . \mathrm{K}^{-1} \cdot \mathrm{mol}^{-1}$, is in agreement with Pauling's entropy, $\frac{R}{2} \ln \left(\frac{3}{2}\right)=1.68 \mathrm{~J} \cdot \mathrm{K}^{-1} \cdot \mathrm{mol}^{-1}[24]$. (a) and (b) also show a comparison with Monte Carlo simulations (MC data) of [25]. This figure was taken from [24].

The first Hamiltonian to discuss the physics of spin ice in good approximation is an effective model with nearest neighbour ferromagnetic interaction between the moments which, together with the strong crystal field, gives rise to a frustrated ferromagnetic system [6]:

$$
\mathcal{H}=-3 \mathrm{~J}_{\mathrm{eff}} \sum_{(i j)} \boldsymbol{S}_{i} \cdot \boldsymbol{S}_{j}
$$

where $\mathrm{J}_{\mathrm{eff}}>0$ is the effective nearest-neighbour coupling of this nearest neighbour spin ice (NNSI) Hamiltonian. According to Harris et al. [19] the ground state of this system is identical to the Pauling model for water ice [21], which presents an extensive residual entropy of ground state and obeys the 
ice rules.

However, the spin-ice materials $\mathrm{Ho}_{2} \mathrm{Ti}_{2} \mathrm{O}_{7}$ and $\mathrm{Dy}_{2} \mathrm{Ti}_{2} \mathrm{O}_{7}$, with rare earth ions $\mathrm{Ho}^{3+}$ and $\mathrm{Dy}^{3+}$, carry a sizeable magnetic moment $\mu$ of $\sim 10 \mu_{B}$ which leads to dipolar interactions that cannot be considered as negligible. Furthermore, because the exchange coupling between the ions is due to $4 \mathrm{f}$ electrons, behind $5 \mathrm{~s}$ and 5 p orbitals, it is very weak $(\sim 1 \mathrm{~K})$ in comparison with other ferromagnets $[17,2]$. Thus, for $\mathrm{Ho}^{3+}$ and $\mathrm{Dy}^{3+}$ the dipolar interactions are on the same energy scale as the exchange interaction [2]. The minimal model of Hamiltonian for a dipolar spin ice (DSI) [25], which includes the terms of nearest-neighbour exchange and magnetic dipole interactions, is

$$
\mathcal{H}=-\mathrm{J} \sum_{(i j)} \boldsymbol{S}_{i} \cdot \boldsymbol{S}_{j}+\mathrm{D} r_{\mathrm{nn}}^{3} \sum_{i>j}\left[\frac{\boldsymbol{S}_{i} \cdot \boldsymbol{S}_{j}}{\left|\boldsymbol{r}_{i j}\right|^{3}}-\frac{3\left(\boldsymbol{S}_{i} \cdot \boldsymbol{r}_{i j}\right)\left(\boldsymbol{S}_{j} \cdot \boldsymbol{r}_{i j}\right)}{\left|\boldsymbol{r}_{i j}\right|^{5}}\right],
$$

where $\mathrm{J}, \mathrm{D}$ and $r_{\mathrm{nn}} \sim 3.5 \AA$ are, respectively, the antiferromagnetic exchange coupling, the dipoledipole coupling and the nearest neighbour distance between rare earth ions, which has been shown to provide a comprehensive quantitative description of spin ice materials [25]. Because the local Ising axes belong to the set of $\langle 111\rangle$ vectors, the effective nearest-neighbour coupling $\mathrm{J}_{\text {eff }}$ of equation (30) can be written as $\mathrm{J}_{\mathrm{eff}}=\mathrm{J}_{\mathrm{nn}}+\mathrm{D}_{\mathrm{nn}}$, with $\mathrm{J}_{\mathrm{nn}}=\mathrm{J} / 3$ and $\mathrm{D}_{\mathrm{nn}}=5 \mathrm{D} / 3$ which shows the equivalence between the NNSI and DSI Hamiltonians $[2,25]$. The estimate of the dipole-dipole coupling D is defined as $\mathrm{D}=\mu_{0} \mu^{2} / 4 \pi r_{\mathrm{nn}}^{3}$, thus for both $\mathrm{Ho}_{2} \mathrm{Ti}_{2} \mathrm{O}_{7}$ and $\mathrm{Dy}_{2} \mathrm{Ti}_{2} \mathrm{O}_{7} \mathrm{D} \sim+1.4 \mathrm{~K}$ and $\mathrm{D}_{\mathrm{nn}}=+2.35$ $\mathrm{K}$. The estimated value of $\mathrm{J}$ was obtained from Monte Carlo simulations and specific heat data in $\mathrm{Dy}_{2} \mathrm{Ti}_{2} \mathrm{O}_{7}$ [25] or from neutron scattering on $\mathrm{Ho}_{2} \mathrm{Ti}_{2} \mathrm{O}_{7}$ [26], with reported values of $\mathrm{J} \sim-3.72 \mathrm{~K}$ for $\mathrm{Dy}_{2} \mathrm{Ti}_{2} \mathrm{O}_{7}$ and $\mathrm{J} \sim-1.65 \mathrm{~K}$ for $\mathrm{Ho}_{2} \mathrm{Ti}_{2} \mathrm{O}_{7}$, confirming antiferromagnetic coupling [25, 26]. From above, the effective nearest-neighbour ferromagnetic couplings $\mathrm{J}_{\text {eff }}=\mathrm{J} / 3+5 \mathrm{D} / 3$ in the NNSI model, are 1.1 and $1.8 \mathrm{~K}$ for $\mathrm{Dy}_{2} \mathrm{Ti}_{2} \mathrm{O}_{7}$ and $\mathrm{Ho}_{2} \mathrm{Ti}_{2} \mathrm{O}_{7}$ respectively.

In summary, the spin ices $\mathrm{Ho}_{2} \mathrm{Ti}_{2} \mathrm{O}_{7}$ and $\mathrm{Dy}_{2} \mathrm{Ti}_{2} \mathrm{O}_{7}$ possess a residual low-temperature entropy consistent with that estimated by Pauling applied to the ferromagnetic nearest-neighbour $\langle 111\rangle$ Ising model on the pyrochlore lattice. Also, in $\mathrm{Ho}_{2} \mathrm{Ti}_{2} \mathrm{O}_{7}$ and $\mathrm{Dy}_{2} \mathrm{Ti}_{2} \mathrm{O}_{7}$ the effective ferromagnetic nearest neighbour coupling between spins, which gives rise to frustration in the spin ice, comes from the dipolar interactions $(\mathrm{D}>0)$ being strong enough to balance the antiferromagnetic exchange $(\mathrm{J}<0)$ $[17,25]$. 


\section{Spin liquid}

Among the series of pyrochlores, there are some that exhibit a more exotic magnetic behaviour called spin liquid. This is known for presenting the most dynamic behaviour in which spins continue to fluctuate and evade order even at the lowest temperature observed [27], resembling the constant motion of molecules within a true liquid. Such a spin liquid is an unusual phenomenon, since it has a non-magnetic ground state that is built from well-formed local moments [27]. This remarkable situation of spin liquids is produced by relaxing the Ising nature of the spins [14]. Thus, considerable fluctuations between degenerate configurations are restored, resulting in a spin liquid state, which is known as a "quantum variant" of spin ices [14]. The spin liquids were first proposed by Anderson in 1973 [28] and since then their theoretical possibility has been in discussion. Two potential candidates for the spin liquid state are the pyrochlores $\mathrm{Tb}_{2} \mathrm{Ti}_{2} \mathrm{O}_{7}$ and $\mathrm{Yb}_{2} \mathrm{Ti}_{2} \mathrm{O}_{7}$.

First, we will consider $\mathrm{Tb}_{2} \mathrm{Ti}_{2} \mathrm{O}_{7}$, which is characterized by an Ising-like anisotropy of the $\mathrm{Tb}^{3+}$ ions along the local $\langle 111\rangle$ axes [29], in a similar way to spin ices $\mathrm{Ho}_{2} \mathrm{Ti}_{2} \mathrm{O}_{7}$ and $\mathrm{Dy}_{2} \mathrm{Ti}_{2} \mathrm{O}_{7}$. Although presenting a Curie-Weiss temperature of $\theta_{\mathrm{CW}} \sim-19 \mathrm{~K}$ which indicates antiferromagnetic interactions [29], $\mathrm{Tb}_{2} \mathrm{Ti}_{2} \mathrm{O}_{7}$ does not enter a long-range ordered state [30]. However, the study of Yasui et al. suggested that $\mathrm{Tb}_{2} \mathrm{Ti}_{2} \mathrm{O}_{7}$ suffered a phase transition to an "ordered state" at $\mathrm{T} \sim 1.5 \mathrm{~K}[31]$. Subsequent results of Gardner et al. concluded that the previous order of $\mathrm{Tb}_{2} \mathrm{Ti}_{2} \mathrm{O}_{7}$ at $\mathrm{T} \sim 1.5 \mathrm{~K}$ is a result of a few spins freezing around defects in the stochiometric crystal structure, and also pointed out a disordered fluctuating ground state with short range spin correlations down to $50 \mathrm{mK}$ [32]. From a theoretical perspective, a proposal by Molavian et al. discussed that $\mathrm{Tb}_{2} \mathrm{Ti}_{2} \mathrm{O}_{7}$ is kind of a quantum spin-ice system where the spin-ice-like correlations remain hidden down to $0.5 \mathrm{~K}$ [33].

On the other hand, for ytterbium titanate $\mathrm{Yb}_{2} \mathrm{Ti}_{2} \mathrm{O}_{7}$ early work from Blöte et al. proposed an ordered magnetic state below $200 \mathrm{mK}$, where a sharp anomaly in the specific heat was observed [34]. Also, Bramwell et al. reported a Curie-Weiss temperature $\theta_{\mathrm{CW}} \sim+0.59 \mathrm{~K}$ indicating a weak ferromagnetic coupling [35]. However, in [36] Hodges et al. found an abrupt change in the fluctuation rate of the $\mathrm{Yb}^{3+}$ spin at $240 \mathrm{mK}$ that did not correspond to the ordered state expected from [34]. Specific heat measurements in powder and crystal samples of $\mathrm{Yb}_{2} \mathrm{Ti}_{2} \mathrm{O}_{7}$ show that the temperature of the phase transition varies significantly between them [37, 38], thus it has been proposed by Ross et al. that the low temperature magnetic state is very sensitive to sample preparation conditions [37]. Some samples show anomalies with the highest temperature observed at $265 \mathrm{mK}$ in a polycrystalline powder samples [38]. Other powder samples show slightly broader anomalies at lower temperatures; $250 \mathrm{mK}$ 
and $214 \mathrm{mK}$ [38]. For crystal samples they present broad humps instead of sharp anomalies and in some cases a mixture of broad hump and sharp peak [37]. This sample dependence is illustrated in figure 2.11 taken from [37], in which the specific heat measurements of $\mathrm{Yb}_{2} \mathrm{Ti}_{2} \mathrm{O}_{7}$ show different kind of anomalies at different temperatures. Currently, the transition in $\mathrm{Yb}_{2} \mathrm{Ti}_{2} \mathrm{O}_{7}$ at $200 \mathrm{mK}$ remains unexplained, but it seems essential that a small number of defects in $\mathrm{Yb}_{2} \mathrm{Ti}_{2} \mathrm{O}_{7}$ have the potential to cause significant changes in such a frustrated system [38].

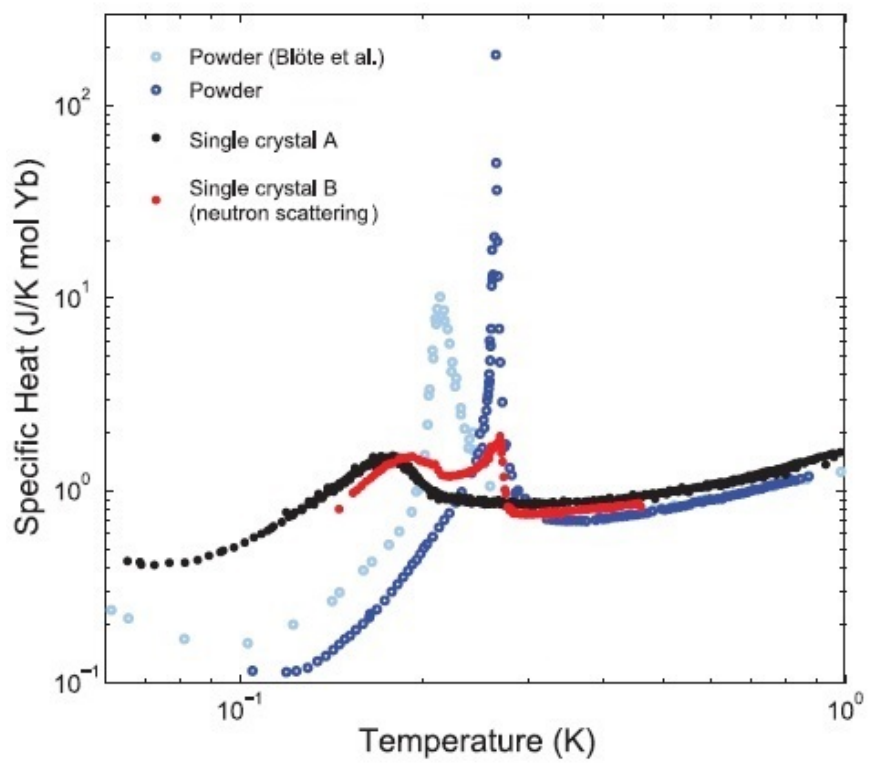

Figure 2.11: Low-temperature specific heat measurements of $\mathrm{Yb}_{2} \mathrm{Ti}_{2} \mathrm{O}_{7}$. A significant sample dependence is observed in both powders and single crystals. The powder sample (blue) shows the highest temperature $(265 \mathrm{mK})$ and sharpest anomaly. The second crystal sample (for neutron scattering purposes), B, shows a sharp peak at $265 \mathrm{mK}$ similar to the powder, and a broad, low-temperature feature similar to crystal A. Figure from [37].

Finally, from this short review of spin liquids $\mathrm{Tb}_{2} \mathrm{Ti}_{2} \mathrm{O}_{7}$ and $\mathrm{Yb}_{2} \mathrm{Ti}_{2} \mathrm{O}_{7}$ it is concluded that the fluctuation of spins and the type of order that might exist are still not well understood and also that in both cases the structural defects have a key role in that unexplained order occurring in them. Thus, further theoretical and experimental studies are required to describe the physics of spin liquids [2]. For a more detailed explanation of the spin liquid state review references [2], [6], and [27]. 


\section{Long-range order}

We will now study rare earth pyrochlores that develop long-range magnetic ordering. These magnetic systems are of significant interest because they provide systems with axial and planar symmetry, quantum order-by-disorder transition, and partially ordered systems [2]. As we discussed earlier, an estimate of the Heisenberg exchange constant $J$ can be computed from the Curie-Weiss temperature $\theta_{\mathrm{CW}}$. For instance, for an ordinary magnet being cooled down to the Curie-Weiss temperature, it is expected that they begin to develop long-range magnetic order. On the other hand, in a magnetic system with the main interaction subject to frustration what happens is that the temperature at which long-range order begins to develop, $\mathrm{T}_{\mathrm{N}}$, decreases to very low temperatures. Examples of rare earth pyrochlores subject to long-range order are $\mathrm{Gd}_{2} \mathrm{Ti}_{2} \mathrm{O}_{7}$ [18] and $\mathrm{Gd}_{2} \mathrm{Sn}_{2} \mathrm{O}_{7}$ [4]. On subsequent Sections we will discuss $\mathrm{Gd}_{2} \mathrm{Ti}_{2} \mathrm{O}_{7}$ deeply when we will study its proposed order structure and also inherent to it the magnetic ordering in $\mathrm{Gd}_{2} \mathrm{Sn}_{2} \mathrm{O}_{7}$.

But before continuing with $\mathrm{Gd}_{2} \mathrm{Ti}_{2} \mathrm{O}_{7}$, in table 2.2 is summarized what it is known about the presence or absence of long-range order in the rare earth pyrochlores $\mathrm{A}_{2} \mathrm{Ti}_{2} \mathrm{O}_{7}$. It shows the number of electrons $\mathrm{n}$ in the $4 \mathrm{f}$ shell of the rare-earth ion, the presence of long-range order, and the temperature of ordering $\mathrm{T}_{c}$. Thus, a first correlation is to notice if the rare-earth ion of the $\mathrm{A}_{2} \mathrm{Ti}_{2} \mathrm{O}_{7}$ pyrochlores have a Kramer ion that is an odd number of electrons. From that, the Kramer ions nearly always show long-range order (being Dy and $\mathrm{Yb}$ exceptions) whereas those with a non-Kramer ion (even electron) do not [39]. Finally, we notice that the existence of a magnetic transition does not automatically implies a long-range order as in the case of $\mathrm{Yb}_{2} \mathrm{Ti}_{2} \mathrm{O}_{7}$ in which the occurrence of long-range order is still in discussion.

\begin{tabular}{c|c|c|c|c}
\hline & Rare earth & n in $4 \mathrm{f}^{n}$ & Long-range order & $\mathrm{T}_{c}(\mathrm{~K})$ \\
\hline \hline $\mathrm{Gd}_{2} \mathrm{Ti}_{2} \mathrm{O}_{7}$ & $\mathrm{Gd}$ & 7 & Yes [40] & 1 \\
\hline $\mathrm{Tb}_{2} \mathrm{Ti}_{2} \mathrm{O}_{7}$ & $\mathrm{~Tb}$ & 8 & No [30] & - \\
\hline $\mathrm{Dy}_{2} \mathrm{Ti}_{2} \mathrm{O}_{7}$ & $\mathrm{Dy}$ & 9 & No [41] & - \\
\hline $\mathrm{Ho}_{2} \mathrm{Ti}_{2} \mathrm{O}_{7}$ & $\mathrm{Ho}$ & 10 & No [42] & - \\
\hline $\mathrm{Er}_{2} \mathrm{Ti}_{2} \mathrm{O}_{7}$ & $\mathrm{Er}$ & 11 & Yes [43] & 1.2 \\
\hline $\mathrm{Yb}_{2} \mathrm{Ti}_{2} \mathrm{O}_{7}$ & $\mathrm{Yb}$ & 13 & Yes [44]/No $[45]$ & 0.2 \\
\hline \hline
\end{tabular}

Table 2.2: Presence or absence of long-range order in the Ti-based pyrochlores. 


\subsection{Gadolinium Titanate $-\mathrm{Gd}_{2} \mathrm{Ti}_{2} \mathrm{O}_{7}$}

In previous Sections we discussed the phenomenon of geometric frustration and the properties of rare-earth pyrochlores in general so that now we are going to focus our study on the pyrochlore oxide $\mathrm{Gd}_{2} \mathrm{Ti}_{2} \mathrm{O}_{7}$ with gadolinium $\mathrm{Gd}^{3+}$ as the central magnetic ion. The rare earth pyrochlore $\mathrm{Gd}_{2} \mathrm{Ti}_{2} \mathrm{O}_{7}$ is regarded as an excellent approximation to a classical Heisenberg antiferromagnet. Indeed, the $\mathrm{Gd}^{3+}$ ion has a half-filled $4 \mathrm{f}-$ shell i.e. an electronic configuration of $4 \mathrm{f}^{7}$ with a large spin angular momentum $S=7 / 2$ and an orbital angular momentum of $L=0$. This configuration evidences that $\mathrm{Gd}^{3+}$ is the best example of a Heisenberg spin among the rare-earth ions. From the Hund's rules [8], the total momentum quantum number is $J=7 / 2$ and therefore its electronic ground state is ${ }^{2 S+1} L_{J}={ }^{8} S_{7 / 2}$.

In $\mathrm{Gd}_{2} \mathrm{Ti}_{2} \mathrm{O}_{7}$, the leading interactions between $\mathrm{Gd}$ spins are the antiferromagnetic nearest-neighbour exchange and the dipole-dipole coupling with additional contributions identified as further nearestneighbour exchanges [18]. However, a considerable anisotropy was found in $\mathrm{Gd}_{2} \mathrm{Ti}_{2} \mathrm{O}_{7}$ [46] and thus a crystal field splitting of the ionic levels occurs due to a strong spin-orbit coupling among $4 \mathrm{f}$ electrons which mixes ${ }^{8} S_{7 / 2}$ and ${ }^{6} P_{7 / 2}$ states $[47,48]$.

For the electronic ground state ${ }^{8} S_{7 / 2}$ of $\mathrm{Gd}_{2} \mathrm{Ti}_{2} \mathrm{O}_{7}$, the total magnetic entropy associated is given by $S=N k_{\mathrm{B}} \ln \left(g_{0}\right)$, where $\mathrm{N}$ is the number of Gd ions and $g_{0}$ is the ground-state degeneracy of $J$. Then, for $J=7 / 2$ the degeneracy is $g_{0}=2 J+1=8$. From the above, the total entropy is $S=N k_{\mathrm{B}} \ln (8)$ and the total entropy per mol of Gd is $S=R \ln (8)$ where $R=k_{\mathrm{B}} N_{\mathrm{A}}$ is the Gas constant and $N_{\mathrm{A}}$ is the Avogadro constant. This value of the expected entropy $S=R \ln (8)=17.3 \mathrm{~J} /(K$.mol $)$ will be discussed in next Section when we will review previous thermal results.

On the experimental side, magnetization measurements of $\mathrm{Gd}_{2} \mathrm{Ti}_{2} \mathrm{O}_{7}$ provide a saturated spinonly moment of $7 \mu_{B}$ per ion of Gd [49] in agreement with the expected value of $g \mu_{B} J=7 \mu_{B}$ where $g=2, J=7 / 2$, and $\mu_{B}$ the Bohr magneton. The magnetic susceptibility for $\mathrm{Gd}_{2} \mathrm{Ti}_{2} \mathrm{O}_{7}$ follows the Curie-Weiss law with an effective magnetic moment close to the theoretical value given by $\mathrm{p}_{\text {eff }}=g \mu_{\mathrm{B}} \sqrt{J(J+1)}=7.94 \mu_{\mathrm{B}}$ and with a Curie-Weiss temperature $\theta_{\mathrm{CW}} \sim-10 \mathrm{~K}$ [18]. Also, it was reported a magnetic phase transition to an ordered state in $\mathrm{Gd}_{2} \mathrm{Ti}_{2} \mathrm{O}_{7}$ when it goes through a temperature of $\mathrm{T}_{\mathrm{N}} \sim 1 \mathrm{~K}[18,50]$. From the above, the frustration index $f \equiv\left|\theta_{\mathrm{CW}}\right| / \mathrm{T}_{\mathrm{N}}$ is $f \sim$ 10. Apart from ac susceptibility measurements which exhibit a transition at $\sim 1 \mathrm{~K}$ [18], subsequent specific heat studies reported two phase transitions in zero applied field, at 0.75 and $1 \mathrm{~K}[39,51]$.

Along with the rare earth pyrochlore $\mathrm{Gd}_{2} \mathrm{Ti}_{2} \mathrm{O}_{7}, \mathrm{Gd}_{2} \mathrm{Sn}_{2} \mathrm{O}_{7}$ is also a good realization of a Heisenberg antiferromagnet [40]. Magnetic properties of the stannate and titanate of gadolinium would 
therefore be expected to be very similar, however, the contrasts between the low-temperature behaviour of $\mathrm{Gd}_{2} \mathrm{Sn}_{2} \mathrm{O}_{7}$ and its analogous $\mathrm{Gd}_{2} \mathrm{Ti}_{2} \mathrm{O}_{7}$ are remarkable [4]. For example, $\mathrm{Gd}_{2} \mathrm{Sn}_{2} \mathrm{O}_{7}$ undergoes a first-order transition into an ordered state near $1 \mathrm{~K}$ different from the two transitions found in $\mathrm{Gd}_{2} \mathrm{Ti}_{2} \mathrm{O}_{7}$ at 0.75 and $1 \mathrm{~K}$ [39]. Another difference between them is the larger lattice constant $a=$ $10.45 \AA$ of $\mathrm{Gd}_{2} \mathrm{Sn}_{2} \mathrm{O}_{7}$ [4] compared with $a=10.184 \AA$ of $\mathrm{Gd}_{2} \mathrm{Ti}_{2} \mathrm{O}_{7}$ [18].

Finally, as we mentioned, the rare earth pyrochlore $\mathrm{Gd}_{2} \mathrm{Ti}_{2} \mathrm{O}_{7}$ exhibit a long range order but this is just part of the story. Hence, in the next Section we will discuss the proposed structures at very low temperatures of the rare earth pyrochlore $\mathrm{Gd}_{2} \mathrm{Ti}_{2} \mathrm{O}_{7}$. These ordered structures are best pictured if the pyrochlore lattice is described by layers stacked along the [111] direction of the crystal [40] as shown on the left side of figure 2.12 taken from [2]. From this perspective, the pyrochlore lattice is seen to consist of alternating Kagomé layers and intermediate triangular planes labeled as the interstitial sites of the lattice [2]. Also, in Chapter 4 we will study the oxygen vacancies in $\mathrm{Gd}_{2} \mathrm{Ti}_{2} \mathrm{O}_{7}$ and the consequences of such crystalline defects on the magnetic ground state. Thus, it is essential to know how the local environment surrounding the rare-earth ions of pyrochlores is, specially the positions of oxygen ions. For $\mathrm{Gd}_{2} \mathrm{Ti}_{2} \mathrm{O}_{7}$ or any other rare earth pyrochlore this is depicted on the right side of figure 2.12, where the six oxygen ions labeled as $\mathrm{O}(2)$ form a ring around a central $\mathrm{Gd}^{3+}$ and the oxygen labeled as $\mathrm{O}(1)$ is inside and lies on the center of the tetrahedra formed of four $\mathrm{Gd}^{3+}$.

(a)

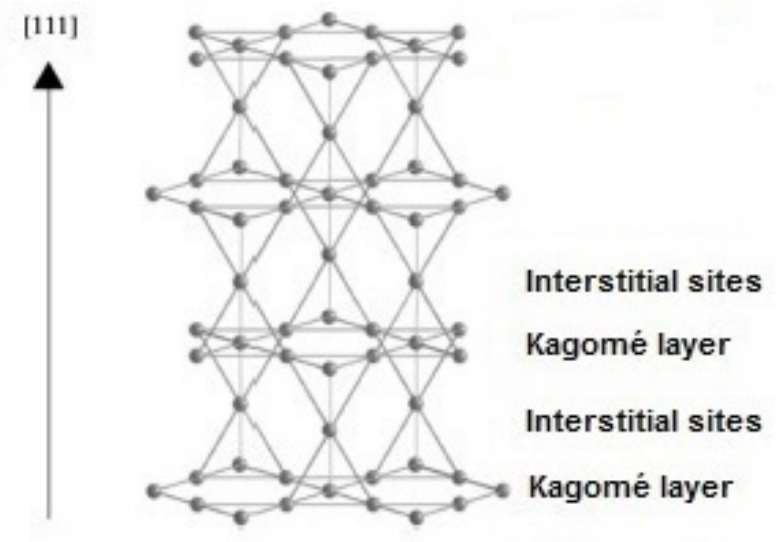

(b)

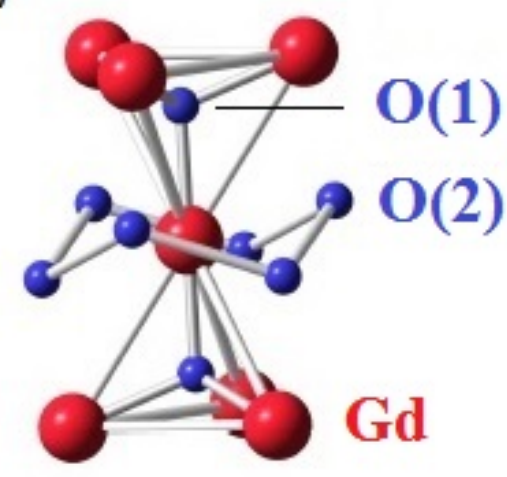

Figure 2.12: (a) The pyrochlore lattice can be visualised as alternating Kagomé and interstitial planar layers stacked along the [111] direction. (b) Corner-sharing tetrahedra of the pyrochlore $\mathrm{Gd}_{2} \mathrm{Ti}_{2} \mathrm{O}_{7}$ with isolated $\mathrm{Gd}$ and $\mathrm{O}$ ions. We have two non-equivalent oxygen at sites $\mathrm{O}(1)$ and $\mathrm{O}(2)$. The Ti ions were removed for clarity. These figures were adapted from [2] and [52]. 


\subsection{Previous work on $\mathrm{Gd}_{2} \mathrm{Ti}_{2} \mathrm{O}_{7}$}

In this Section we are going to present results of previous researches concerning $\mathrm{Gd}_{2} \mathrm{Ti}_{2} \mathrm{O}_{7}$ that propose its magnetic structure of ground state at very low temperatures. Until last year (2014), the accepted proposal for the magnetic structure of $\mathrm{Gd}_{2} \mathrm{Ti}_{2} \mathrm{O}_{7}$ at $50 \mathrm{mk}$ was an ordering of spins known as $4-k$ (which we will explain below) reported on J. R. Stewart et al.'s paper [40]. In the 7th International Conference on Highly Frustrated Magnetism 2014, J. R. Stewart stated that the $4-k$ ordering is inconsistent with new data obtained from new neutron diffraction experiments in $\mathrm{Gd}_{2} \mathrm{Ti}_{2} \mathrm{O}_{7}$, hence ruling out his previous proposal. Then, since it is still under discussion the lowtemperature magnetic ground state of the Heisenberg pyrochlore antiferromagnetic $\mathrm{Gd}_{2} \mathrm{Ti}_{2} \mathrm{O}_{7}$, we will study on this dissertation its ordering at temperatures near to the two magnetic transitions found. Indeed, a clear understanding of the magnetic properties of $\mathrm{Gd}_{2} \mathrm{Ti}_{2} \mathrm{O}_{7}$ will lead us to come up with ideas for its behaviour in presence of crystalline defects as we will study in Chapters 4 and 5 .

\subsubsection{Long-range magnetic order in $\mathrm{Gd}_{2} \mathrm{Ti}_{2} \mathrm{O}_{7}$}

The problem of antiferromagnetic nearest-neighbour exchange interactions on the pyrochlore lattice was first considered by Anderson (1956) in the spinel structure of ferrites, where he predicted a very high ground state degeneracy and that no long range order would exist at any temperature for Ising spins [53]. More than twenty years later, Villain (1979) reached the same conclusion for Heisenberg spins with antiferromagnetic nearest-neighbour interactions on the pyrochlore lattice [54]. Villain stated that such system will display an unusual collective behaviour which remained disordered and fluctuating down to zero temperature, giving rise to a state that he called collective paramagnet [54]. However, the best example of an Heisenberg antiferromagnet on a pyrochlore lattice: $\mathrm{Gd}_{2} \mathrm{Ti}_{2} \mathrm{O}_{7}$ enters into an unexpected long-range ordered state at low temperatures differing from that collectiveparamagnet behaviour [18].

Raju et al. (1999) reported in [18] the dc magnetic susceptibility $\chi$ vs temperature for a polycrystalline sample of $\mathrm{Gd}_{2} \mathrm{Ti}_{2} \mathrm{O}_{7}$. The collected data was found to follow the Curie-Weiss behaviour in the range $10 \mathrm{~K}-300 \mathrm{~K}$ as is shown in figure 2.13 from [18], with an effective magnetic moment of 7.7 $\mu_{B} / \mathrm{Gd}$ ion and a Curie-Weiss temperature $\theta_{\mathrm{CW}}$ of $-9.6 \mathrm{~K}$ indicating antiferromagnetic interactions between the Gd spins. 


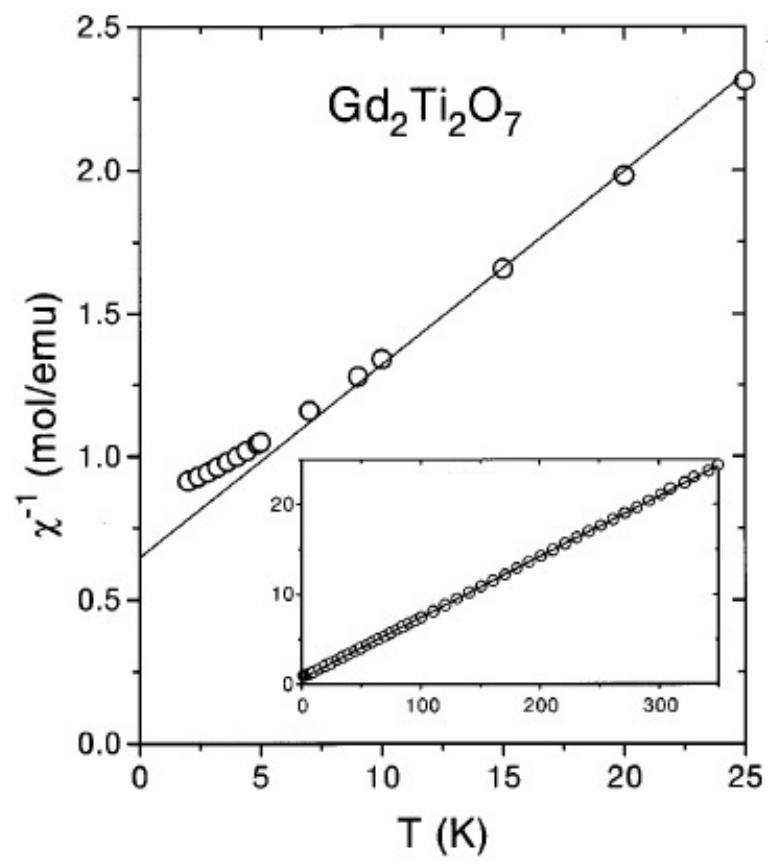

Figure 2.13: Inverse susceptibility $\chi^{-1}$ of $\mathrm{Gd}_{2} \mathrm{Ti}_{2} \mathrm{O}_{7}$ against temperature in the range $2-25 \mathrm{~K}$, and in the range $2-300 \mathrm{~K}$ in the inset. Figure taken from [18].

In the same paper, Raju et al. observed from experimental data of ac susceptibility $\chi_{\mathrm{ac}}$ a broad peak centered near to $1 \mathrm{~K}$ as show in figure 2.14, which provides an indication of a transition to longrange antiferromagnetic order [18]. Also, measurements of $\chi_{\mathrm{ac}}(\omega)$ seem to be independent of frequency [18] as shown in 2.14. The specific heat $\mathrm{C}_{\mathrm{m}}$ as a function of temperature is shown in figure 2.14. Here, there is a single sharp peak at $\mathrm{T}=0.97 \mathrm{~K}$ (better illustrated in the inset) in agreement with the ac asusceptibility data developing a transition to a long-range-ordered state [18]. The specific heat from $1.4 \mathrm{~K}$ to $18 \mathrm{~K}$ shows a broad Schottky anomaly peaked near $2 \mathrm{~K}$ with magnetic contributions extending to $20 \mathrm{~K}$. The solid line in figure 2.14 represents the theoretical fit of the Schottky peak which is detailed in [18]. 

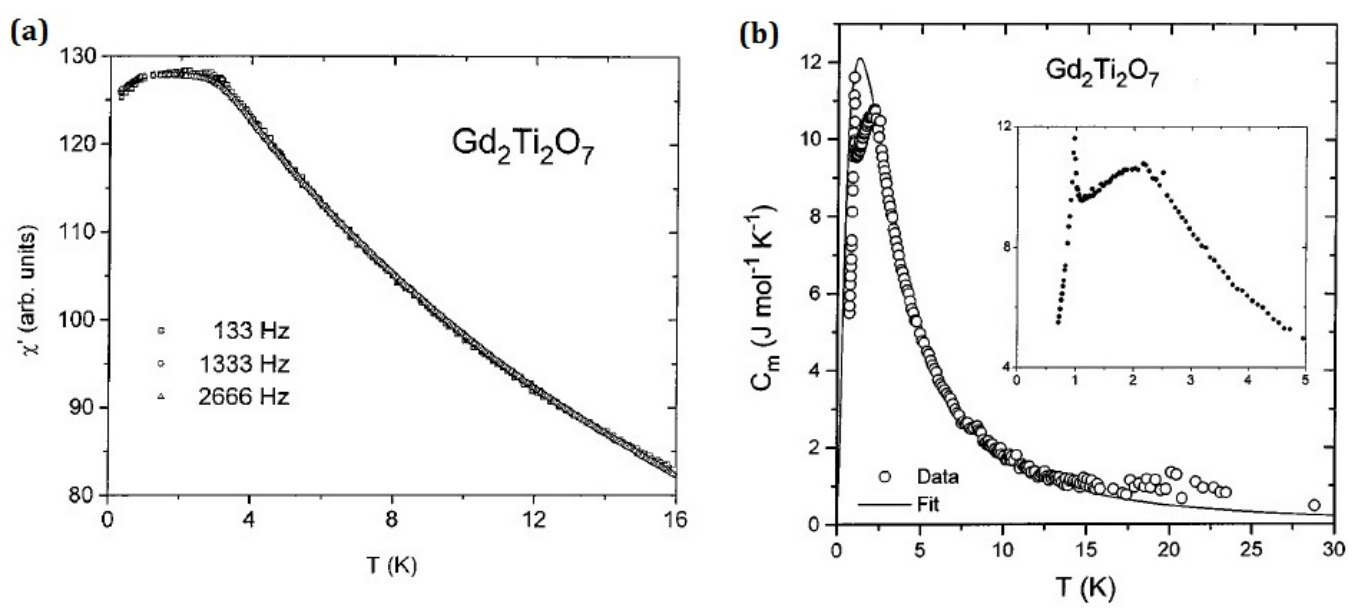

Figure 2.14: (a) Real part of the ac susceptibility $\chi^{\prime}$ vs temperature at different frequencies, (b) specific heat $\mathrm{C}_{\mathrm{m}}$ vs temperature. The inset displays $\mathrm{C}_{\mathrm{m}}$ in the low-temperature range below $5 \mathrm{~K}$. Figures taken from [18].

To establish a model for the magnetic-ordered phase of the spins in pyrochlores systems many previous works have made use of the mean field theory (MFT) $[55,56]$. The formalism of this theory is beyond the scope of this dissertation however we will discuss about some order parameters obtained from MFT that can be compared with experimental results specifically with neutron diffraction. In very simple terms, calculations of MFT imply first to consider a Hamiltonian $\mathcal{H}$ for the spins $\mathbf{S}_{i}$ in the system and from it analyse the thermodynamic of the system through its free energy $F$ which has the form

$$
F=\operatorname{Tr}(\rho \mathcal{H})+k_{\mathrm{B}} T \operatorname{Tr}(\rho \ln \rho),
$$

where $\rho$ is the full density matrix of the system, $\mathrm{T}$ is the temperature, and $\mathcal{H}$ the Hamiltonian of the spins $\mathbf{S}_{i}$. In the paper of Reimers et al. [55] is set the procedure to obtain an expansion for the free energy $F$ of the system in terms of the order parameters $\mathbf{B}\left(\mathbf{r}_{\mathrm{i}}\right)$ at lattice sites $\mathbf{r}_{i}$ (for a detailed definition review [55]). Also, in this calculation it is mandatory that the order parameters $\mathbf{B}\left(\mathbf{r}_{\mathbf{i}}\right)$ are expanded in terms of Fourier components as

$$
\mathbf{B}\left(\mathbf{r}_{i}\right)=\sum_{\mathbf{q}} \mathbf{B}(\mathbf{q}) \exp \left(i \mathbf{q} \cdot \mathbf{r}_{i}\right)
$$

This results in a diagonalization problem of the free energy $F$ to calculate the ground state and the transition temperature as a function of the order parameter $\mathbf{q}$ [55]. In the MFT $\mathbf{q}$ (also labeled as $\mathbf{k}$ ) 
is defined as the ordering vector correlated to the ground state of the spins [55]. On the experimental side, $\mathbf{q}$ is an essential result of neutron diffraction characterizing the ordered structures of spins at low temperatures, thus a comparison between experiment and theory has been discussed in previous works as we will see below.

Within the framework of the MFT, Raju et al. discussed in [18] what the expected magnetic properties and type of magnetic-ordered phase for $\mathrm{Gd}_{2} \mathrm{Ti}_{2} \mathrm{O}_{7}$ are. First, they considered the classical spin Hamiltonian $\mathcal{H}$ with nearest-neighbour antiferromagnetic interactions and dipole-dipole coupling between spins:

$$
\mathcal{H}=-\mathrm{J} \sum_{(i j)} \boldsymbol{S}_{i} \cdot \boldsymbol{S}_{j}+\mathrm{D} r_{\text {nn }}^{3} \sum_{(i j)}\left[\frac{\boldsymbol{S}_{i} \cdot \boldsymbol{S}_{j}}{r_{i j}^{3}}-\frac{3\left(\boldsymbol{S}_{i} \cdot \boldsymbol{r}_{i j}\right)\left(\boldsymbol{r}_{i j} \cdot \boldsymbol{S}_{j}\right)}{r_{i j}^{5}}\right],
$$

where $\mathrm{J}, \mathrm{D}$ and $r_{\mathrm{nn}}$ are, respectively, the antiferromagnetic exchange coupling, the dipolar coupling and the nearest neighbour distance between $\mathrm{Gd}^{3+}$ ions. On [18] is set an estimate of the nearestneighbour exchange $\mathrm{J} \sim-4.8 \mathrm{~K}$ and a strength of $\mathrm{D} \sim 0.8 \mathrm{~K}$ for the dipole-dipole exchange. With this Hamiltonian, Raju et al. followed the line set in [55] and showed that $\mathcal{H}$ does not lift the ground state degeneracy to second order in the expansion of the free energy. However, it embraces an ordering vector $\mathbf{q}=\left(\begin{array}{lll}h & h & h\end{array}\right)[18]$. Thus, in the frustrated magnet $\mathrm{Gd}_{2} \mathrm{Ti}_{2} \mathrm{O}_{7}$ Raju et al. stated that the longrange order is not established for nearest-neighbour antiferromagnetic interactions $\mathrm{J}$ and long-range dipolar interactions D only [18]. Instead, long-range order is expected when we also have in account a second $\left(\mathrm{J}_{2}\right)$ or third $\left(\mathrm{J}_{3}\right)$ nearest-neighbour exchange interactions [18].

Finally, Raju et al. concluded that the frustrated $\mathrm{Gd}_{2} \mathrm{Ti}_{2} \mathrm{O}_{7}$ exhibits a transition to a long-rangeordered state at $0.97 \mathrm{~K}$ and that the hamiltonian of the pyrochlore $\mathrm{Gd}_{2} \mathrm{Ti}_{2} \mathrm{O}_{7}$ must described nearestneighbour and dipolar interactions, and also exchange beyond nearest neighbours $\mathrm{J}_{2}$ and $\mathrm{J}_{3}$.

\subsubsection{Palmer-Chalker State in the pyrochlore antiferromagnet}

Palmer and Chalker (2000) studied in [57] the problem of the pyrochlore antiferromagnet including dipole-dipole interactions just as Raju et al. had discussed [18]. However, they extended the meanfield description of this system with the previous Hamiltonian $\mathcal{H}$ to find an ordering pattern below a finite temperature T. Thus, they showed that the quartic term in the expansion of the free energy lifts the ground state degeneracy, finding the ground state for spins on the pyrochlore antiferromagnet with the Hamiltonian $\mathcal{H}$ [57]. With this fourth-order expansion, the spins on the pyrochlore lattice select an ordering wave vector $\mathbf{q}=(000)$ known as the Palmer-Chalker state $(\mathrm{PC})$. 
The Palmer-Chalker state $\mathbf{q}=(000)$ is shown in figure 2.15 for spins on a single tetrahedron of the pyrochlore. The ground state for the full pyrochlore lattice is a periodic repetition of that for a single tetrahedron. In figure 2.15 taken from [58] all the spins lie on the $x y$ plane and form pairs of antiparallel spins that are parallel to the opposite edge of the tetrahedron they belong to. According to Palmer and Chalker the ordering obtained is due to energy selection and does not occur by an entropically selected mechanism [57].

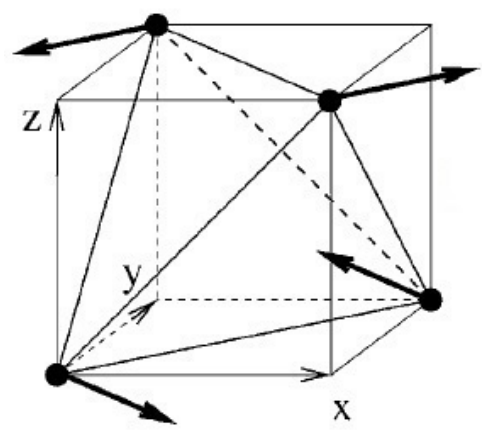

Figure 2.15: The PC state $\mathbf{q}=(000)$ for a single tetrahedron of the pyrochlore lattice. Here, all the spins lie onto the $x y$ plane and are parallel to certain edge of the tetrahedron. Figure taken from [58]

Figure 2.16 from [57] shows the configuration of the spins on the $x y$ plane for the PC state with ordering vector $\mathbf{q}=(000)$. Here, the spins are coplanar and form what is called a four-sublattice Néel state [57].

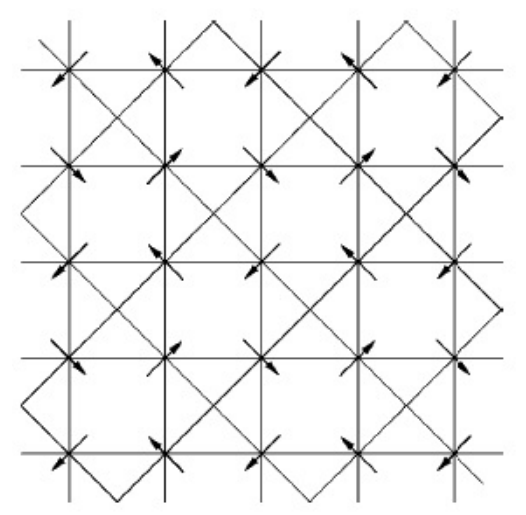

Figure 2.16: Projection of the PC state $\mathbf{q}=(000)$ onto the $x y$ plane of the pyrochlore lattice. Figure taken from [57]. 
In the study of the ground state in the geometrically frustrated lattices, it is worthwhile to mention that the ordering vector $\mathbf{q}=\mathbf{0}$ defines a different ground state of the spins in the frustrated Kagomé lattice. We cite this because, as we mentioned before, the pyrochlore lattice could be understood as parallel layers of Kagomé planes with atoms in interstitial positions along the [111] direction. Thus, the ground state in the Kagomé lattice could provide us a hint of the spin configuration in the pyrochlore. In [56] Reimers et al. showed that for spins in the Kagomé lattice interacting by nearest-neighbour antiferromagnetic exchange, the system stabilizes an ordering vector $\mathbf{q}=\mathbf{0}$. In this configuration the three spins on the triangular sublattices forming an arrange of $120^{\circ}$ among them as shown in figure 2.17. This is known as the ground state $\mathbf{q}=\mathbf{0}$ for spins in a antiferromagnet Kagomé lattice.

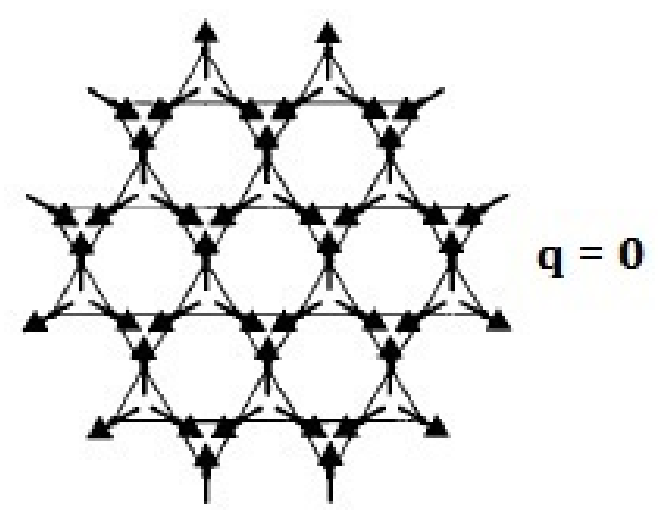

Figure 2.17: The ground state $\mathbf{q}=\mathbf{0}$ for spins in the Kagomé lattice. The spins on each triangle order in a $120^{\circ}$ pattern. This figure was taken from [59].

\subsubsection{Low-temperature magnetic properties of $\mathrm{Gd}_{2} \mathrm{Ti}_{2} \mathrm{O}_{7}$}

Bonville et al. (2003) reported in [39] specific heat, Mössbauer, magnetic susceptibility and magnetization measurements for the antiferromagnet pyrochlores $\mathrm{Gd}_{2} \mathrm{Ti}_{2} \mathrm{O}_{7}$ and $\mathrm{Gd}_{2} \mathrm{Sn}_{2} \mathrm{O}_{7}$. However, we will discuss here the temperature dependence of the specific heat and results from Mössbauer spectroscopy for these pyrochlores. To carry out these experiments on $\mathrm{Gd}_{2} \mathrm{Ti}_{2} \mathrm{O}_{7}$ and $\mathrm{Gd}_{2} \mathrm{Sn}_{2} \mathrm{O}_{7}$, Bonville et al. employed polycrystalline samples grown by the method described in [39]. In addition to these results, we will discuss the magnetic susceptibility data reported by Petrenko et al. in [60]. It is important to point out that for these experiments the authors of [60] worked with single crystal samples of $\mathrm{Gd}_{2} \mathrm{Ti}_{2} \mathrm{O}_{7}$ instead of polycrystalline samples as in [39]. 
For $\mathrm{Gd}_{2} \mathrm{Ti}_{2} \mathrm{O}_{7}$, Bonville et al. reported the specific heat data divided by the temperature as shown in figure 2.18. It shows clearly two peaks corresponding to two phase transitions in $\mathrm{Gd}_{2} \mathrm{Ti}_{2} \mathrm{O}_{7}$, the first one is at $\mathrm{T}_{\mathrm{N}}^{1}=1.05 \mathrm{~K}$ and the second is at $\mathrm{T}_{\mathrm{N}}^{2}=0.75 \mathrm{~K}$. Also, the amplitudes of both peaks are of an order of magnitude $\sim 10 \mathrm{~J} . \mathrm{K}^{-2} \cdot \mathrm{mol}^{-1}$. Then, according to Bonville et al. $\mathrm{Gd}_{2} \mathrm{Ti}_{2} \mathrm{O}_{7}$ enters in a long-range order state at low temperatures as stated earlier by Raju et al. in [18]. By integrating the specific heat data divided by the temperature, Bonville et al. found the magnetic entropy of $\mathrm{Gd}_{2} \mathrm{Ti}_{2} \mathrm{O}_{7}$ which is shown by the solid line in the inset of figure 2.18. Also, as we showed in Section 2.5, the total magnetic entropy at high temperatures for $\mathrm{Gd}_{2} \mathrm{Ti}_{2} \mathrm{O}_{7}$ with $J=7 / 2$ is $S=R \ln (8)=17.3 \mathrm{~J} . \mathrm{K}^{-1} \cdot \mathrm{mol}^{-1}$ which is represented by the dash line in the inset of figure 2.18. About the $20 \%$ and $35 \%$ of the total magnetic entropy of $R \ln (8)$ is released up to the temperatures of the two transitions $\mathrm{T}_{\mathrm{N}}^{1} \sim 1 \mathrm{~K}$ and $\mathrm{T}_{\mathrm{N}}^{2} \sim 0.75 \mathrm{~K}$ [39]. This value of entropy is not fully recovered for temperatures above $5 \mathrm{~K}$ indicating the presence of considerable short-range order interactions [39].

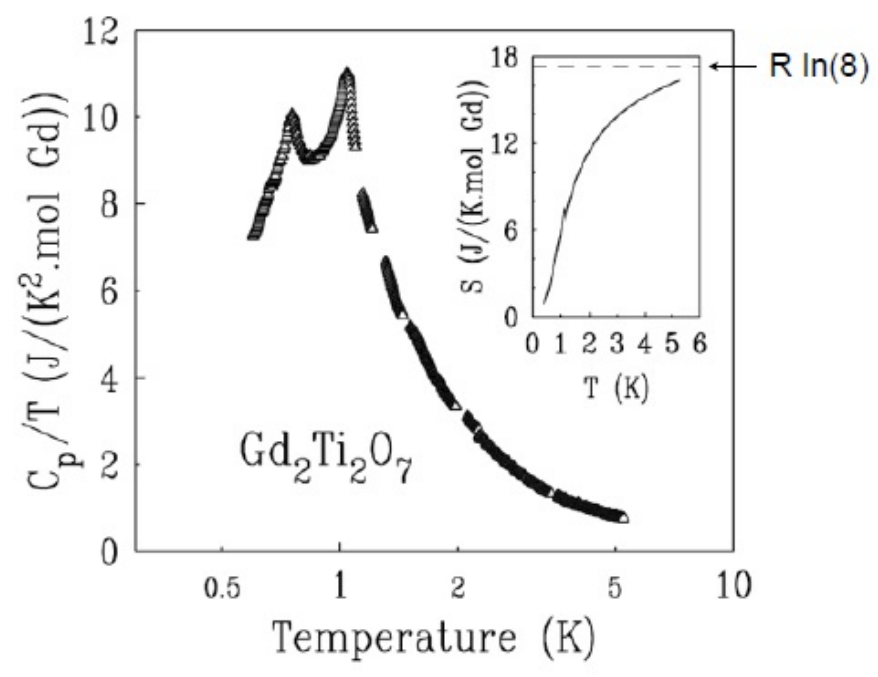

Figure 2.18: Thermal dependence of the specific heat divided by the temperature of $\mathrm{Gd}_{2} \mathrm{Ti}_{2} \mathrm{O}_{7}$. The inset shows the change in the entropy and the dashed line represents the total magnetic entropy $R \ln (8)=17.3 \mathrm{~J} \cdot \mathrm{K}^{-1} \cdot \mathrm{mol}^{-1}$. This figure was taken from [39].

Bonville et al. also reported the specific heat data versus temperature for $\mathrm{Gd}_{2} \mathrm{Sn}_{2} \mathrm{O}_{7}$ as shown in figure 2.19 from [39]. Here, there is a single sharp peak at $\mathrm{T}_{\mathrm{N}}=1.015 \mathrm{~K}$ [39]. The amplitude of the peak of $\mathrm{Gd}_{2} \mathrm{Sn}_{2} \mathrm{O}_{7}$ is $\sim 120 \mathrm{~J} . \mathrm{K}^{-1} \cdot \mathrm{mol}^{-1}$ which is of an order of magnitude higher compared with the peaks of $\mathrm{Gd}_{2} \mathrm{Ti}_{2} \mathrm{O}_{7}$. Also, in the inset of figure 2.19 the dashed line represents the total magnetic 
entropy $R \ln (8)=17.3 \mathrm{~J} \cdot \mathrm{K}^{-1} \cdot \mathrm{mol}^{-1}$. The inset shows that about the $40 \%$ of the total magnetic entropy is released up to the temperature of the transition $\mathrm{T}_{\mathrm{N}}=1.015 \mathrm{~K}$. Thus, for $\mathrm{Gd}_{2} \mathrm{Sn}_{2} \mathrm{O}_{7}$ short-range interactions are present in higher temperatures just as in $\operatorname{Gd}_{2} \operatorname{Ti}_{2} \mathrm{O}_{7}$.

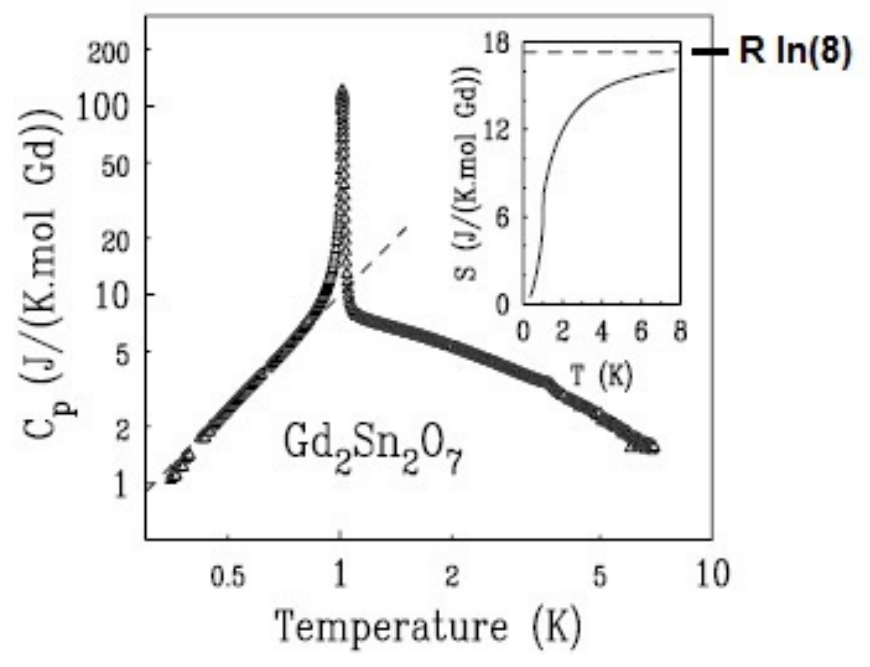

Figure 2.19: Specific heat as a function of temperature for $\mathrm{Gd}_{2} \mathrm{Sn}_{2} \mathrm{O}_{7}$. The inset shows the variation in entropy and the dashed lines represent $R \ln (8)=17.3 \mathrm{~J} \cdot \mathrm{K}^{-1} \cdot \mathrm{mol}^{-1}$.

Mössbauer measurements reported by Bonville et al. in [39] discuss the orientation of the magnetic moments in the pyrochlore materials $\mathrm{Gd}_{2} \mathrm{Ti}_{2} \mathrm{O}_{7}$ and $\mathrm{Gd}_{2} \mathrm{Sn}_{2} \mathrm{O}_{7}$. As mentioned before, in each tetrahedron of the pyrochlore lattice the rare-earth $\mathrm{Gd}^{3+}$ ions lie at their respective vertices. These $\mathrm{Gd}^{3+}$ ions or magnetic moments have a saturated low temperature value of $7 \mu_{B}$ and also they have as symmetry axis the local $\langle 111\rangle$ direction defined from the Gd site to the center of the tetrahedron [39]. From Mössbauer measurements, in $\mathrm{Gd}_{2} \mathrm{Sn}_{2} \mathrm{O}_{7}$ at temperatures $\sim 1 \mathrm{~K}$, the four magnetic moments of the tetrahedra have the same size $\left(\sim 5.25 \mu_{B}\right)$ and each is perpendicular to the local $\langle 111\rangle$ axis [39]. On the other hand, for $\mathrm{Gd}_{2} \mathrm{Ti}_{2} \mathrm{O}_{7}$ at temperatures between the transitions that is between $0.75 \mathrm{~K}$ and $1 \mathrm{~K}$ reveals that three sites of the tetrahedron retain an orientation perpendicular to the local direction $\langle 111\rangle$ and the fourth site has a tilted and different sized moment [39]. In terms of the magnetic structure, Bonville et al. stated that those three sites of the tetrahedron correspond to unchanged spin orientations in the Kagomé layer, with a different moment in the interstitial sites. This is illustrated in figure 2.20 taken from [50]. Thus, in a very summarized idea, according to the Mössbauer measurements in $\mathrm{Gd}_{2} \mathrm{Ti}_{2} \mathrm{O}_{7}$ and $\mathrm{Gd}_{2} \mathrm{Sn}_{2} \mathrm{O}_{7}$, each magnetic moment on the pyrochlore 
lattice lies perpendicular to its local trigonal axis $\langle 111\rangle$.

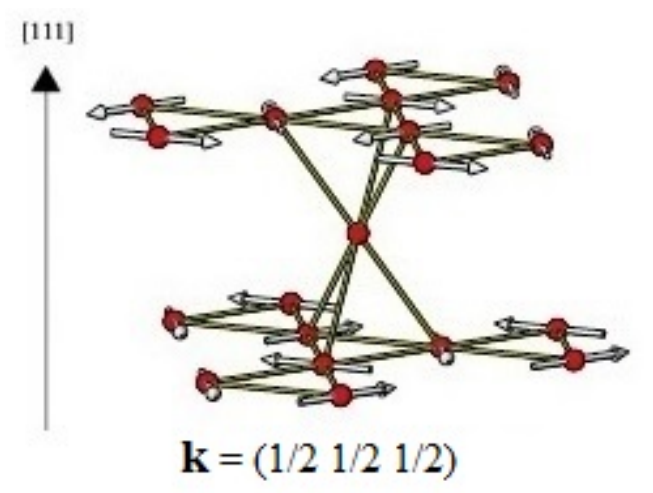

Figure 2.20: Spin orientations in the Kagomé planes are perpendicular to crystalline direction [111] and the disordered spins lie in the interstitial sites. The so-called $1-k$ model. Figure from [50].

Before ending this Section, we are going to present magnetic susceptibility results of $\mathrm{Gd}_{2} \mathrm{Ti}_{2} \mathrm{O}_{7}$ reported by Petrenko et al. in [60]. Figure 2.21(a) from [60] shows the temperature dependence of the magnetic susceptibility of $\mathrm{Gd}_{2} \mathrm{Ti}_{2} \mathrm{O}_{7}$ measured with a magnetic field of 100 Oe applied along the [100] direction. Here, the arrows indicate the two transition temperatures at $1.02 \mathrm{~K}$ and $0.74 \mathrm{~K}$ in agreement with the specific heat measurements in zero field of [39] and [60]. Also, the second transition at $0.74 \mathrm{~K}$ is slightly clear even though the sample was a $\mathrm{Gd}_{2} \mathrm{Ti}_{2} \mathrm{O}_{7}$ crystal. Thus, if for the crystal samples the transition peaks are not so clear, we expect that in polycrystalline samples of $\mathrm{Gd}_{2} \mathrm{Ti}_{2} \mathrm{O}_{7}$ the transitions from susceptibility measurements will be difficult to distinguish. Figure 2.21(b) shows the temperature dependence of the susceptibility measured in different magnetic fields. Here, for low magnetic fields as $0.93 \mathrm{kOe}$ and $4.66 \mathrm{kOe}$ we can resolve two transitions near to $1.02 \mathrm{~K}$ and 0.74 K. However, for a field of $56.9 \mathrm{kOe}$ the values of magnetic susceptibility are still increasing until the lowest shown temperature $0.5 \mathrm{~K}$, suggesting thus a magnetic transition under this temperature. These curves are consecutively offset by $0.05 \mathrm{emu} / \mathrm{mol}$ for clarity. 
(a)

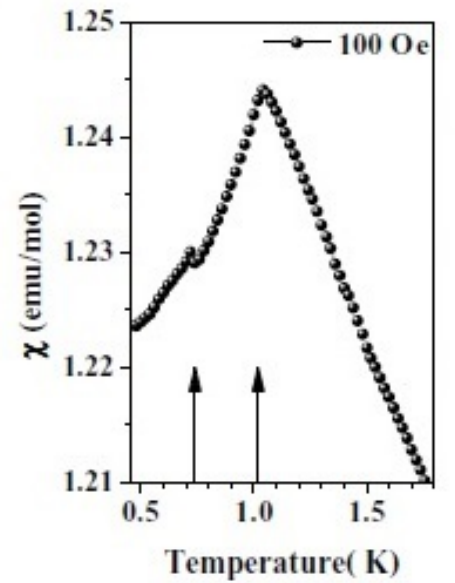

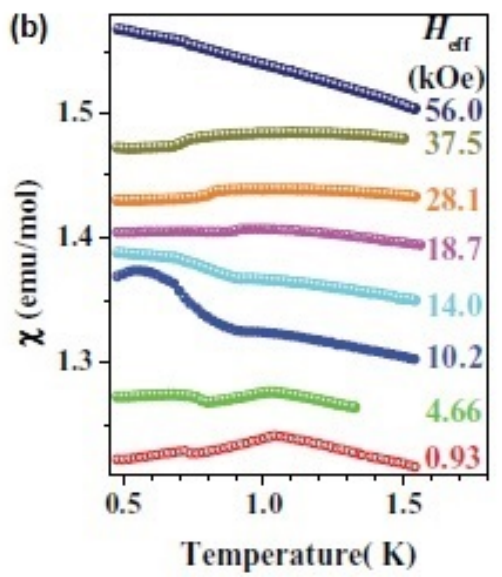

Figure 2.21: (a) Temperature dependence of the magnetic susceptibility of $\mathrm{Gd}_{2} \mathrm{Ti}_{2} \mathrm{O}_{7}$ measured with a magnetic field of $100 \mathrm{Oe}$, the arrows indicate the two transitions at 1.02 and $0.74 \mathrm{~K}$. (b) Temperature dependence of the susceptibility measured for different magnetic fields. Figure taken from [60].

\subsubsection{Neutron diffraction and multi-k order in $\mathrm{Gd}_{2} \mathrm{Ti}_{2} \mathrm{O}_{7}$}

The ordered magnetic structures at low temperatures in pyrochlore materials can be studied directly by neutron diffraction experiments. However, in $\mathrm{Gd}_{2} \mathrm{Ti}_{2} \mathrm{O}_{7}$ and $\mathrm{Gd}_{2} \mathrm{Sn}_{2} \mathrm{O}_{7}$ these experiments are difficult to carry out since gadolinium is very adverse to them [2]. Because of the high absorption cross section of gadolinium ${ }^{155} \mathrm{Gd}$, neutron studies are performed in isotopically enriched ${ }^{160} \mathrm{Gd}_{2} \mathrm{Ti}_{2} \mathrm{O}_{7}$ samples [50]. In fact, the difficulty of performing neutron scattering on $\mathrm{Gd}_{2} \mathrm{Ti}_{2} \mathrm{O}_{7}$ has led to reconsider its proposed structure at very low temperatures as we will discuss here.

First studies of neutron diffraction in $\mathrm{Gd}_{2} \mathrm{Ti}_{2} \mathrm{O}_{7}$ were performed by Champion et al. (2001) and reported in [50]. They found that the magnetic structure below $\mathrm{T}_{\mathrm{N}}^{1} \sim 1 \mathrm{~K}$ is indexed with an ordering vector $\mathbf{k}=\left(\frac{1}{2} \frac{1}{2} \frac{1}{2}\right)$. This $\mathbf{k}$ agrees with the ordering vector of $\mathrm{Gd}_{2} \mathrm{Ti}_{2} \mathrm{O}_{7}$ predicted by Raju et al. [18]. The spin structure observed for the pyrochlore $\mathrm{Gd}_{2} \mathrm{Ti}_{2} \mathrm{O}_{7}$ can be understood in terms of the Kagomé planes and interstitial sites forming the pyrochlore as shown in figure 2.12. The ground state $\mathbf{k}=$ $\left(\frac{1}{2} \frac{1}{2} \frac{1}{2}\right)$ for $\mathrm{Gd}_{2} \mathrm{Ti}_{2} \mathrm{O}_{7}$ at $50 \mathrm{mK}$ consists of the ordering $\mathbf{q}=\mathbf{0}$ for the Kagomé planes that means an arrangement of $120^{\circ}$ between the spins of the triangular sublattices (figure 2.17) with the spins in the interstitial sites carrying a either statically or dynamically disordered spin as shown in figure 2.20. This result implies that about $1 / 4$ of Gd spins are disordered at $50 \mathrm{mK}$. As stated by Champion et 
al., neutron diffraction can be ambiguous for high symmetry systems so there is always the possibility of multi- $k$ structures. These are alternative structures described by one or several symmetry-related ordering vectors $k$ which give identical diffraction patterns [40] as we will discuss below. To sum up, the dipolar Heisenberg antiferromagnet $\mathrm{Gd}_{2} \mathrm{Ti}_{2} \mathrm{O}_{7}$ is partially ordered at $50 \mathrm{mK}$ in a pattern that consists of $\mathbf{q}=\mathbf{0}$ Kagomé planes plus a disordered interstitial moment [50].

Stewart et al. (2004) discussed the problem of the multi- $k$ structures in $\operatorname{Gd}_{2} \mathrm{Ti}_{2} \mathrm{O}_{7}$ by neutron diffraction experiments [40]. First, as expected, they found the neutron diffraction pattern below the first transition at $\mathrm{T}_{\mathrm{N}}^{1} \sim 1 \mathrm{~K}$ and the ordering vector $\mathbf{k}=\left(\frac{1}{2} \frac{1}{2} \frac{1}{2}\right)$. Also, the data was consistent with the magnetic structure of $\mathrm{Gd}_{2} \mathrm{Ti}_{2} \mathrm{O}_{7}$ described in the previous paragraph, which was labeled as the $1-k$ model. On it, $1 / 4$ of Gd spins are disordered while $3 / 4$ of spins are fully ordered [40]. The second transition at $\mathrm{T}_{\mathrm{N}}^{2} \sim 0.75 \mathrm{~K}$ is associated with a partial order on the disordered site that is the interstitial spin [40]. Stewart et al. stated that the $1-k$ structure generates multi- $k$ possibilities: $2-k, 3-k$ and $4-k$ which give identical diffraction patterns [40]. Nevertheless, the $2-k$ and $3-k$ were first rule out because they provided unphysical magnetic moments of $\mathrm{Gd}^{3+}$ greater than $7 \mu_{\mathrm{B}}$. Thus, Stewart et al. retained the $1-k$ and $4-k$ to work with.

The $1-k$ and $4-k$ structures are shown in figure 2.22 from [40]. In the $1-k$ structure, the ordered spins lie on the Kagomé planes perpendicular to the single global [111] crystallographic direction and in between these planes the spins remain disordered [40]. These disordered spins have a weak moment in first approximation [40]. On the other hand, for the $4-k$ structure the spins are perpendicular to the local trigonal $\langle 111\rangle$ axes, which connect the vertices of the tetrahedra with their centres [40]. On it, there is essentially a disordered tetrahedron. In addition to that, the disordered spins in both structures lie in different lattice sites. In the $1-k$ structure, nearest neighbour disordered spins are separated by a much larger distance of $7.2 \AA$ compared with $3.6 \AA$ of the $4-k$ structure [40]. 


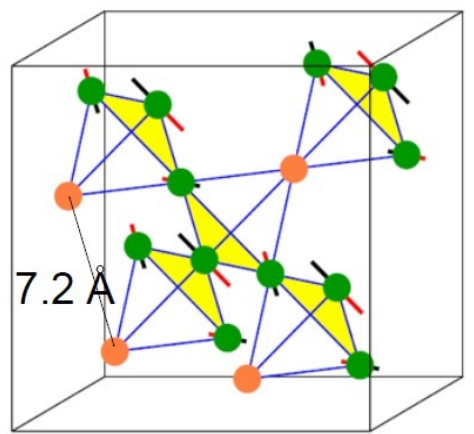

$1-\mathrm{k}$ model

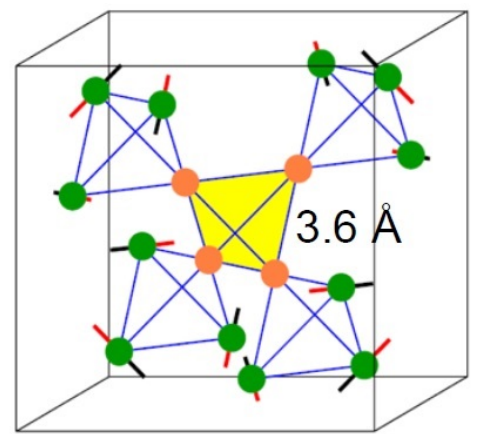

4-k model

Figure 2.22: Low temperature magnetic structures $1-k$ and $4-k$ of $\mathrm{Gd}_{2} \mathrm{Ti}_{2} \mathrm{O}_{7}$. In each model the four disordered Gd spins are coloured in orange. The phase transition at $\mathrm{T}_{\mathrm{N}}^{2} \sim 0.75 \mathrm{~K}$ involves partial ordering of these four spins. Figure from [40].

The same comparison between the $1-k$ and $4-k$ structures is pictured in figure 2.23 from [62]. On it, it is more clear the ordered Kagomé plane (blue) with the disordered spins (yellow) for the $1-k$ structure.

(a)

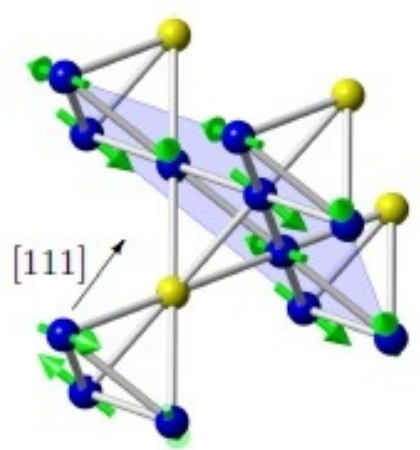

(b)

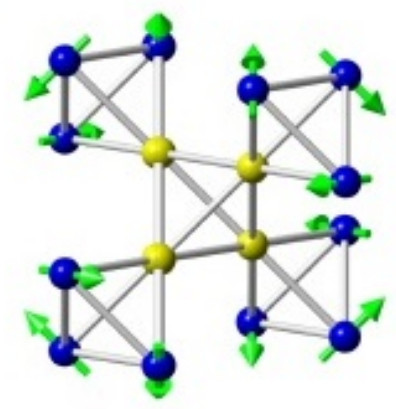

Figure 2.23: (a) $1-k$ structure along the direction [111], the ordered sites (blue) form a $120^{\circ}$ pattern on a triangle in a Kagomé plane and the disordered spins lie on the interstitial sites (yellow). (b) $4-k$ structure with the ordered spins being perpendicular to the local $\langle 111\rangle$ axes. Figure taken from [62].

Reported data of diffuse neutron scattering by Stewart et al. is shown in figure 2.24 from [40]. They analysed the diffuse neutron scattering because it is able to distinguish the differences between the $1-k$ and $4-k$ structures [40]. Then, the diffuse neutron pattern was modeled involving first and 
second near-neighbour interactions [40]. In figure 2.24, the $1-k$ and $4-k$ fits are shown as the red and blue lines respectively, and of which the $4-k$ model produces a better fit than the $1-k$ model. Thus, Stewart et al. stated that for $\mathrm{Gd}_{2} \mathrm{Ti}_{2} \mathrm{O}_{7}$ the magnetic structure at $46 \mathrm{mK}$ was the $4-k$ model [40].

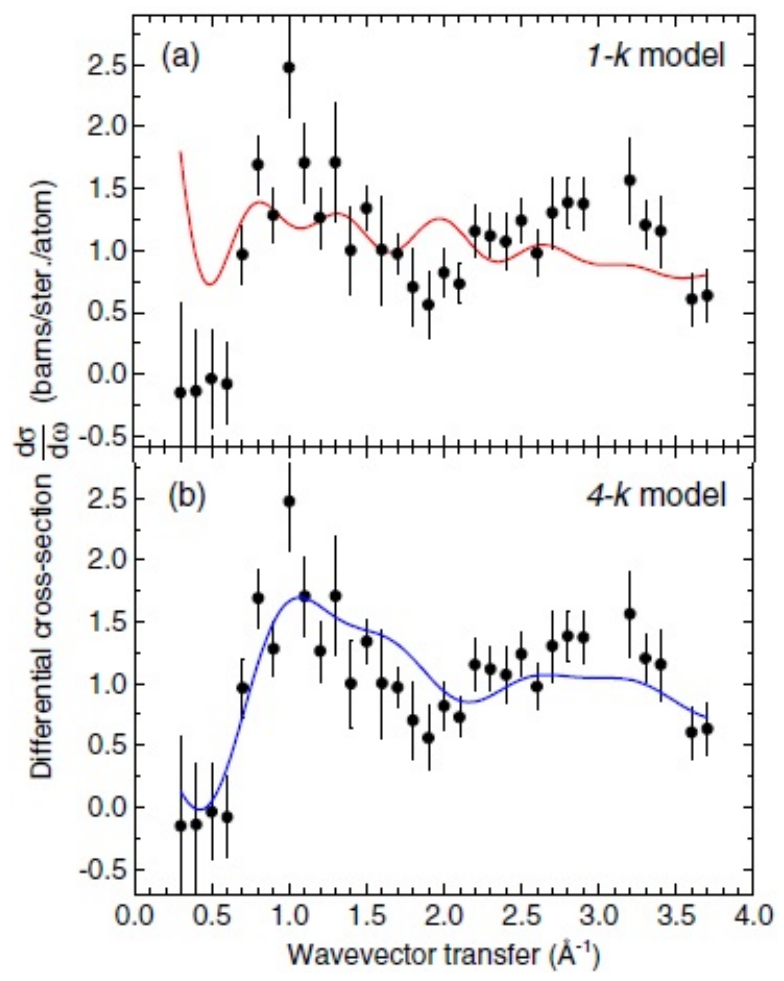

Figure 2.24: Magnetic diffuse scattering at $46 \mathrm{mK}$ (black circles). Fits of the data with $1-k$ (a) and $4-k$ (b) models. This figure was taken from [40].

\subsubsection{Magnetic ordering in $\mathrm{Gd}_{2} \mathrm{Sn}_{2} \mathrm{O}_{7}$ and $\mathrm{Gd}_{2} \mathrm{Ti}_{2} \mathrm{O}_{7}$}

Wills et al. (2006) reported that $\mathrm{Gd}_{2} \mathrm{Sn}_{2} \mathrm{O}_{7}$ orders with the PC ground state which is the result of having as leading interactions the antiferromagnetic exchange and dipole-dipole coupling. This PC State, $\mathbf{q}=(000)$, differs largely from the ground state of its closely related $\mathrm{Gd}_{2} \mathrm{Ti}_{2} \mathrm{O}_{7}, \mathbf{k}=\left(\frac{1}{2} \frac{1}{2} \frac{1}{2}\right)$, described above. Thus, Wills et al. stated that the magnetic Hamiltonian of $\mathrm{Gd}_{2} \mathrm{Ti}_{2} \mathrm{O}_{7}$ contains additional terms [4]. In figure 2.25(a) from [4] is shown the second-neighbour exchange $\mathrm{J}_{2}$ as well as the two types of third-neighbour exchange $J_{31}$ and $J_{32}$. Then, from MFT, Wills et al. determined the instability of the ordering vector for different values of second- and third-neighbour exchange 
constants. This is shown in figure 2.25(b) from [4] which shows the diagram of possible ordering vectors for a small regime of $\mathrm{J}_{31}$ and $\mathrm{J}_{2}$. Wills et al. found that antiferromagnetic interactions $\mathrm{J}_{31}$ stabilizes the order vector $\mathbf{k}=\left(\frac{1}{2} \frac{1}{2} \frac{1}{2}\right)$, which exists in the range of $\mathrm{J}_{31}$ between 0 and $0.335 \mathrm{~J}$. Also, as expected, they found that the ground state for $\mathrm{Gd}_{2} \mathrm{Ti}_{2} \mathrm{O}_{7}: \mathbf{k}=\left(\frac{1}{2} \frac{1}{2} \frac{1}{2}\right)$ corresponds to a $120^{\circ}$ spin structure with $\mathbf{q}=\mathbf{0}$ in the Kagomé planes and no ordered spins on the interstitial sites.

(a)

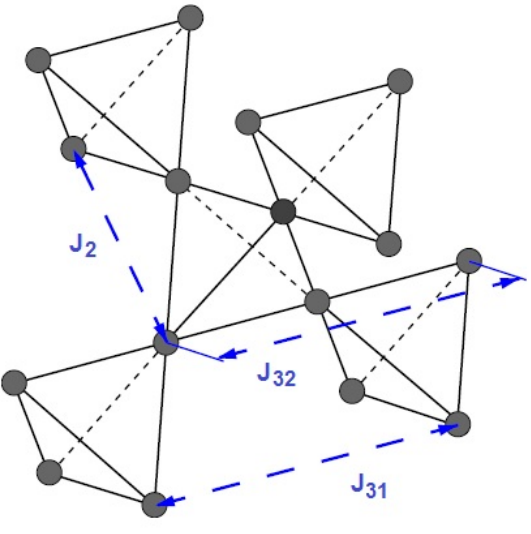

(b)

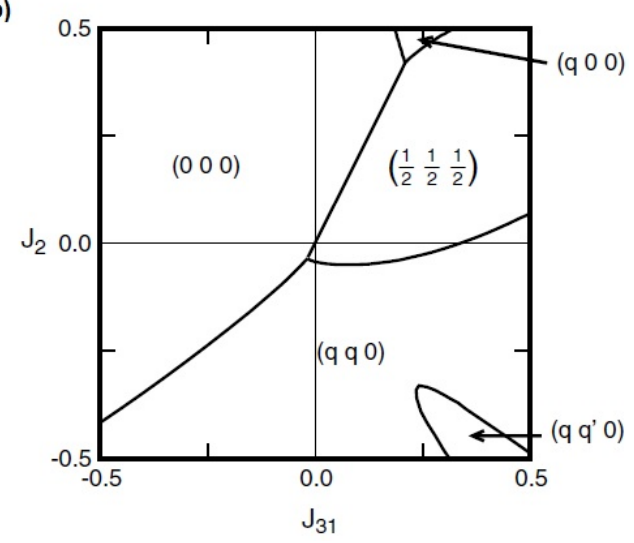

Figure 2.25: (a) Pyrochlore lattice of corner-sharing tetrahedra where next-neighbour exchanges are shown by dashed lines. (b) Ordering wavevectors for different values of second- and third- neighbour exchange constants for a Heisenberg antiferromagnet with dipolar interactions. Figures from [4].

To sum up, the fact that $\mathrm{Gd}_{2} \mathrm{Sn}_{2} \mathrm{O}_{7}$ orders with the PC State indicates how excellent it is to model the Heisenberg pyrochlore antiferromagnet with dipolar interactions. On the other hand, the Hamiltonian of $\mathrm{Gd}_{2} \mathrm{Ti}_{2} \mathrm{O}_{7}$ possesses additional interactions as a type of third-neighbour exchange which led to the magnetic orderings $1-k$ and $4-k$ mentioned in the previous Section.

\subsubsection{The low temperature magnetic ground state of $\mathrm{Gd}_{2} \mathrm{Ti}_{2} \mathrm{O}_{7}$ is not $4-k$}

Taking place in the 7th International Conference on Highly Frustrated Magnetism 2014, J. R. Stewart and J. Paddison stated that the previously $4-k$ structure was ruled out as a candidate for the low temperature magnetic ground state at $50 \mathrm{mK}$. They revealed that the $4-k$ structure is not consistent with the new data of in-field neutron diffraction [61]. They also cited the work of Javanparast et al. (2013) in which by introducing different bilinear interactions to the Hamiltonian of $\mathrm{Gd}_{2} \mathrm{Ti}_{2} \mathrm{O}_{7}$ it is studied the problem of partially ordered phases with periodically arranged disordered sites on 
the pyrochlore lattice. Figure 2.26 from [62] shows the order selected depending of the interaction constants in the Hamiltonian. From it, Javanparast et al. predicted a $1-k$ or $4-k$ structure to exist in $\mathrm{Gd}_{2} \mathrm{Ti}_{2} \mathrm{O}_{7}$ with the order vector $\mathbf{k}=\left(\frac{1}{2} \frac{1}{2} \frac{1}{2}\right)$ between the temperatures $\mathrm{T}_{\mathrm{N}}^{1}=1 \mathrm{~K}$ and $\mathrm{T}_{\mathrm{N}}^{2}=0.75$ $\mathrm{K}$ [62]. In conclusion, J. Paddison et al. stated the $1-k$ structure for $\mathrm{Gd}_{2} \mathrm{Ti}_{2} \mathrm{O}_{7}$ is still valid with the latest research [61].

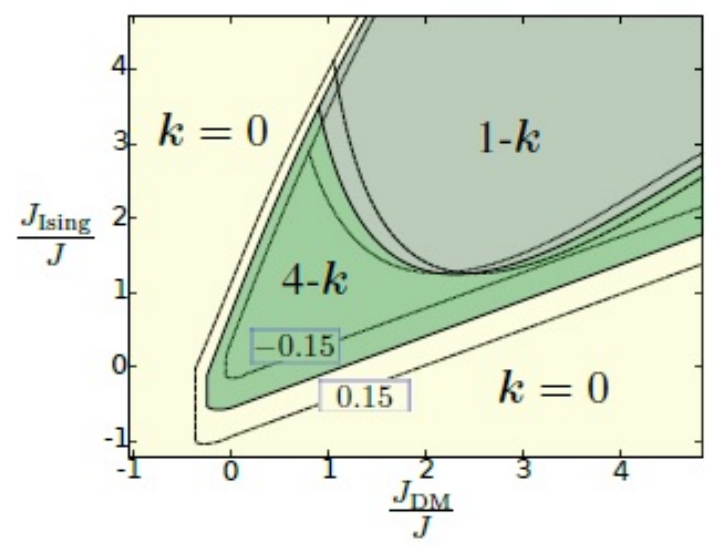

Figure 2.26: Ordering vectors at $\mathrm{T}_{\mathrm{N}}^{1}=1 \mathrm{~K}$ obtained from MFT [62]. The combined area $1-k$ and $4-k$ correspond to the order vector $\mathbf{k}=\left(\frac{1}{2} \frac{1}{2} \frac{1}{2}\right)$. The $\mathbf{k}=\mathbf{0}$ region involve all states for which all spins are fully ordered. Figure taken from [62].

\subsubsection{Vacancy defects in pyrochlores}

Sala et al. discussed in [63] that the vacancy defects of oxygen are the main cause of magnetic impurities in as-grown samples of the pyrochlores $\mathrm{Y}_{2} \mathrm{Ti}_{2} \mathrm{O}_{7}$ and spin ice $\mathrm{Dy}_{2} \mathrm{Ti}_{2} \mathrm{O}_{7}$. They reported the effects of the oxygen vacancies in the structure and magnetism of $\mathrm{Y}_{2} \mathrm{Ti}_{2} \mathrm{O}_{7}$ and $\mathrm{Dy}_{2} \mathrm{Ti}_{2} \mathrm{O}_{7}$ using neutron scattering and magnetization measurements.

For $\mathrm{Y}_{2} \mathrm{Ti}_{2} \mathrm{O}_{7}$, Sala et al. analysed the $\mathrm{x}$-ray diffraction data of different samples: oxygen-depleted, as-grown, and annealed in oxygen $\mathrm{Y}_{2} \mathrm{Ti}_{2} \mathrm{O}_{7}$. Then, they reported that these samples refined in the pyrochlore with space group $F d \overline{3} m$. The refinement revealed equal occupancies on the $\mathrm{Y}$ and Ti sites minimizing thus the stuffing of Ti sites by Y. Also, Sala et al. reported that for the depleted $\mathrm{Y}_{2} \mathrm{Ti}_{2} \mathrm{O}_{7}$ the majority of the vacancies were found to be on the $\mathrm{O}(1)$ sites [63]. As shown in [63] the length of the unit cell increases monotonically with decreasing oxygen concentration. Sala et al. modeled the defect structure due to the presence of oxygen vacancies which corresponds to a distortion of the pyrochlore 
lattice shown in figure 2.27 from [63]. Here, there are $\mathrm{Y}$ ions that relax away from the vacancy $\mathrm{O}(1)$ owing to Coulomb repulsion, two $\mathrm{Ti}^{4+}$ ions transform to $\mathrm{Ti}^{3+}$ to preserve charge neutrality, and also neighbour $\mathrm{O}(2)$ ions move towards $\mathrm{Ti}^{4+}$ ions to give the correct bond lengths [63].

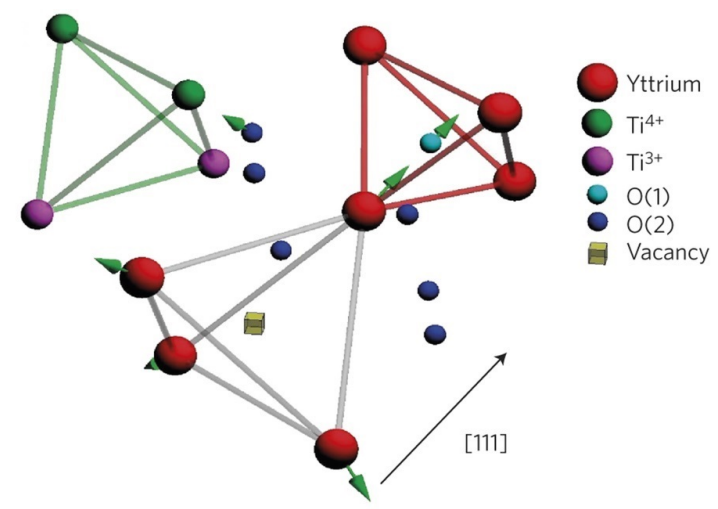

Figure 2.27: Defect structure in the pyrochlore $\mathrm{Y}_{2} \mathrm{Ti}_{2} \mathrm{O}_{7-\delta}$ around an $\mathrm{O}(1)$ vacancy defect. The displacements are displayed by green arrows. Figure adapted from [63].

For $\mathrm{Dy}_{2} \mathrm{Ti}_{2} \mathrm{O}_{7}$, Sala et al. reported that annealing as-grown $\mathrm{Dy}_{2} \mathrm{Ti}_{2} \mathrm{O}_{7}$ in oxygen led to defect-free crystals concluding that the dominant defects are also oxygen vacancies [63]. These oxygen vacancies are located on the $\mathrm{O}(1)$ sites according to neutron scattering studies [63]. Sala et al. reported a comparison between the static magnetic susceptibility of an as-grown $\mathrm{Dy}_{2} \mathrm{Ti}_{2} \mathrm{O}_{7}$ sample before and after annealing in oxygen. This measurement showed a clearly reduction in saturation magnetization that implies a reduced moment on the defective $\mathrm{Dy}^{3+}$ sites [63]. Thus, the oxygen vacancies reduce the saturation magnetization [63]. On the other hand, Sala et al. reported for $\mathrm{Dy}_{2} \mathrm{Ti}_{2} \mathrm{O}_{7}$ crystal electric field calculations [64]. They showed that the $\mathrm{Dy}^{3+}$ ions have a reduced moment in the presence of and $\mathrm{O}(1)$ vacancy and that the anisotropy of the moments change [64]. Recalling the ground-state of the spin ice in which the magnetic moments have an easy axis along $\langle 111\rangle$, in the oxygen-depleted spin ice $\mathrm{Dy}_{2} \mathrm{Ti}_{2} \mathrm{O}$ the magnetic moments lie in a easy plane perpendicular to the local $\langle 111\rangle[64]$.

Thus, the results of [63] make it clear that the density of oxygen vacancies in spin-ice samples or another frustrated system is of key importance in the understanding of their magnetic properties at low temperatures. We will recall this ideas in Chapter 4 when we will study the effects of oxygen vacancies in $\mathrm{Gd}_{2} \mathrm{Ti}_{2} \mathrm{O}_{7}$. 


\subsubsection{Summary}

To end this Chapter, it is convenient to sum up the proposed structure at low temperatures of $\mathrm{Gd}_{2} \mathrm{Ti}_{2} \mathrm{O}_{7}$ concluded from experimental and theoretical studies of the works above-mentioned. Thus, according to the authors in each work:

- Raju et al. (1999)

- The main terms in the Hamiltonian of the Heisenberg pyrochlore $\mathrm{Gd}_{2} \mathrm{Ti}_{2} \mathrm{O}_{7}$ are the nearestneighbour antiferromagnetic exchange and dipolar interaction.

- Long-range order in $\mathrm{Gd}_{2} \mathrm{Ti}_{2} \mathrm{O}_{7}$ is expected to occur including interactions beyond nearestneighbour exchange $\mathrm{J}$ and dipole-dipole coupling $\mathrm{D}$.

- From mean field theory, these interactions stabilizes an ordering vector $\mathbf{q}=(h h h)$.

- Palmer and Chalker (2000)

- The Heisenberg pyrochlore antiferromagnet with dipolar interactions lift the degeneracy and stabilize a four-sublattice state using an approximation to the quartic terms in the free energy.

- It is stabilized an ordering vector $\mathbf{q}=(000)$ corresponding to the ground state known as the Palmer-Chalker state.

- Bonville et al. (2003)

- Specific heat data of $\mathrm{Gd}_{2} \mathrm{Ti}_{2} \mathrm{O}_{7}$ shows two magnetic transitions at $\mathrm{T}_{\mathrm{N}}^{1}=1 \mathrm{~K}$ and $\mathrm{T}_{\mathrm{N}}^{2}=$ $0.75 \mathrm{~K}$. For $\mathrm{Gd}_{2} \mathrm{Sn}_{2} \mathrm{O}_{7}$, there is a phase transition at $1 \mathrm{~K}$.

- Mössbauer experiments evidence in $\mathrm{Gd}_{2} \mathrm{Ti}_{2} \mathrm{O}_{7}$ and $\mathrm{Gd}_{2} \mathrm{Sn}_{2} \mathrm{O}_{7}$ the magnetic moments lie perpendicular to the local trigonal $\langle 111\rangle$ axis.

- Stewart et al. (2004)

- From powder neutron diffraction, the magnetic structure of $\mathrm{Gd}_{2} \mathrm{Ti}_{2} \mathrm{O}_{7}$ corresponds to the ordering vector $\mathbf{k}=\left(\frac{1}{2} \frac{1}{2} \frac{1}{2}\right)$.

- Proposed structures: $1-k$ and $4-k$ (figure 2.22 ). 
- Wills et al. (2006)

- $\mathrm{Gd}_{2} \mathrm{Sn}_{2} \mathrm{O}_{7}$ orders in the PC State with the ordering vector $\mathbf{q}=(000)$ (figure 2.15).

- From MFT, additional terms in the Hamiltonian of $\mathrm{Gd}_{2} \mathrm{Ti}_{2} \mathrm{O}_{7}$ are a type of third-neighbour exchange $J_{31}$. It stabilizes the ordering vector $\mathbf{k}=\left(\frac{1}{2} \frac{1}{2} \frac{1}{2}\right)$.

- Paddison et al. (2015)

- The previous low-temperature $4-k$ model was ruled out because it does not reconcile with in-field neutron diffraction experiments.

- The $1-k$ model at low temperatures is still valid.

- It is predicted a $1-k$ or $4-k$ model between the temperatures $\mathrm{T}_{\mathrm{N}}^{1}=1 \mathrm{~K}$ and $\mathrm{T}_{\mathrm{N}}^{2}=0.75 \mathrm{~K}$ by considering additional terms in the interaction Hamiltonian of $\mathrm{Gd}_{2} \mathrm{Ti}_{2} \mathrm{O}_{7}$. 


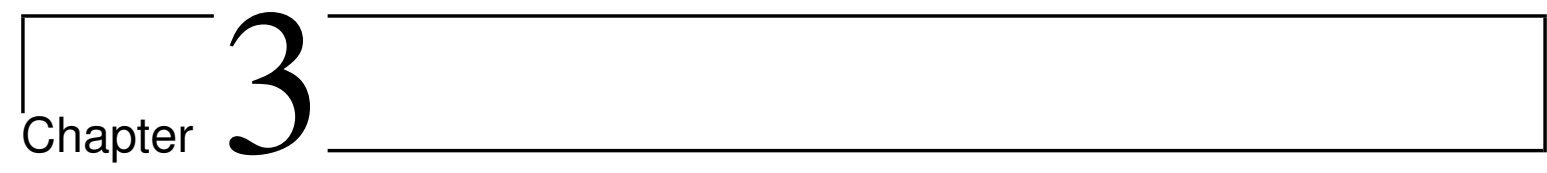

\section{Sample Preparation and Experimental}

\section{Techniques}

In this chapter we describe the growth of polycrystalline powder samples of $\mathrm{Gd}_{2} \mathrm{Ti}_{2} \mathrm{O}_{7}$ and diluted samples of $\mathrm{Gd}_{2} \mathrm{Ti}_{2} \mathrm{O}_{7}$ with yttrium Y: $\mathrm{Gd}_{2-x} \mathrm{Y}_{x} \mathrm{Ti}_{2} \mathrm{O}_{7}$. For the preparation of the samples it was employed an alternative route known as the sol-gel method. We describe the procedure to obtain polycrystalline as-grown $\mathrm{Gd}_{2} \mathrm{Ti}_{2} \mathrm{O}_{7}$ samples which are the starting point to study vacancy defects in different annealing conditions. Also, the sol-gel method with specific variations on the preparation provides polycrystalline diluted $\mathrm{Gd}_{2-x} \mathrm{Y}_{x} \mathrm{Ti}_{2} \mathrm{O}_{7}$ samples with different yttrium contents $x$.

We study X-ray diffraction technique in order to determine the crystalline structure of our samples. To quantify the vacancy defects and the yttrium dilution in $\mathrm{Gd}_{2} \mathrm{Ti}_{2} \mathrm{O}_{7}$ and $\mathrm{Gd}_{2-x} \mathrm{Y}_{x} \mathrm{Ti}_{2} \mathrm{O}_{7}$ respectively, we employ the Rietveld refinement using the FullProf software.

Furthermore, we explain the experiments performed to study magnetically and thermally the $\mathrm{Gd}_{2} \mathrm{Ti}_{2} \mathrm{O}_{7}$ and $\mathrm{Gd}_{2-x} \mathrm{Y}_{x} \mathrm{Ti}_{2} \mathrm{O}_{7}$ polycrystalline samples. Magnetic measurements were carried out on a Vibrating Sample Magnetometer (VSM), an AC susceptometer, and a Superconducting Quantum Interference Device (SQUID), whereas specific heat experiments were performed on a Physical Property Measurement System (PPMS). 


\subsection{Sol-gel method}

Most of the works reviewed in Chapter 2 concerning the pyrochlore $\mathrm{Gd}_{2} \mathrm{Ti}_{2} \mathrm{O}_{7}$ employ different methods to prepare stoichiometric samples. Two methods widely used to prepare polycrystalline powder and crystal samples of $\mathrm{Gd}_{2} \mathrm{Ti}_{2} \mathrm{O}_{7}$ are the solid-state reaction method and the optical floating zone technique respectively. Using the conventional method of solid-state reaction, a single phase polycrystalline sample of $\mathrm{Gd}_{2} \mathrm{Ti}_{2} \mathrm{O}_{7}$ is prepared by mixing and grinding the constituent oxide powders: $\mathrm{Gd}_{2} \mathrm{O}_{3}$ and $\mathrm{TiO}_{2}$. Then, by heat treatment of the mixture several times up to $1350^{\circ} \mathrm{C}$ with intermediate grindings for a few days, the polycrystalline powder of $\mathrm{Gd}_{2} \mathrm{Ti}_{2} \mathrm{O}_{7}$ is synthesized [39]. The second method; the optical floating zone; uses as starting material a pressed powder prepared by solid-state reaction to synthesize the single crystal [65]. The procedures of these methods are described in detail in the literature $[39,65]$.

However, in this dissertation it was employed an alternative route known as the sol-gel method to prepare polycrystalline samples of $\mathrm{Gd}_{2} \mathrm{Ti}_{2} \mathrm{O}_{7}$ and $\mathrm{Gd}_{2-x} \mathrm{Y}_{x} \mathrm{Ti}_{2} \mathrm{O}_{7}$. Indeed, this technique has been used in the synthesis of other pyrochlores like $\mathrm{Y}_{2} \mathrm{Ti}_{2} \mathrm{O}_{7}$ [66] and diluted $\mathrm{Gd}_{2-2 x} \mathrm{Bi}_{2 x} \mathrm{Ti}_{2} \mathrm{O}_{7}$ [67]. The sol-gel method starts from precursor dissolutions, in which metal ions are distributed, and then during the heat treatment, the metal oxide powders are left [66]. In comparison with powder samples prepared by solid-state reaction, the sol-gel method allows us to obtain powders extremely pure that are mixed at a molecular level [66]. Thus, this method allows us to achieve a high chemical homogeneity with a shorter reaction time and lower temperatures of heat treatment [67]. In addition, it is worthwhile to mention that the preparation of diluted samples of $\mathrm{Gd}_{2-x} \mathrm{Y}_{x} \mathrm{Ti}_{2} \mathrm{O}_{7}$ by the sol-gel method is a practical process to introduce ions (in our case $\mathrm{Y}^{3+}$ ) in the structure using an appropriate precursor.

In next Subsections we describe the sol-gel method used to prepare polycrystalline powder samples of $\mathrm{Gd}_{2} \mathrm{Ti}_{2} \mathrm{O}_{7}$ and $\mathrm{Gd}_{2-x} \mathrm{Y}_{x} \mathrm{Ti}_{2} \mathrm{O}_{7}$ that we will discuss in the Chapters 4 and 5 respectively.

\subsubsection{Polycrystalline growth of $\mathrm{Gd}_{2} \mathrm{Ti}_{2} \mathrm{O}_{7}$}

The polycrystalline samples of $\mathrm{Gd}_{2} \mathrm{Ti}_{2} \mathrm{O}_{7}$ were prepared at the Institute of Chemistry of the University of São Paulo by Dr. Flavio Vichi, doctoral student Marina Leite, and with my assistance. The sol-gel method employed was based on the synthesis described in [66]. To synthesize the polycrystalline powder of $\mathrm{Gd}_{2} \mathrm{Ti}_{2} \mathrm{O}_{7}$ it was used gadolinium oxide powder $\mathrm{Gd}_{2} \mathrm{O}_{3}$ (99.99\%, Reacton) and alkoxide tetrabutyl titanate $\mathrm{Ti}\left(\mathrm{OC}_{4} \mathrm{H}_{9}\right)_{4}$ (Gelest) -also called $\mathrm{Ti}(\mathrm{OBu})_{4}-$ as starting materials. Both $\mathrm{Gd}_{2} \mathrm{O}_{3}$ and $\mathrm{Ti}(\mathrm{OBu})_{4}$ were used as the precursors of Gd and Ti respectively. The stoichiometry of the chemical 
reaction is given by

$$
\mathrm{Gd}_{2} \mathrm{O}_{3}+2 \mathrm{TiO}_{2} \rightarrow \mathrm{Gd}_{2} \mathrm{Ti}_{2} \mathrm{O}_{7}
$$

First, according to [66], a non-stoichiometric amount of powder citric acid $\mathrm{C}_{6} \mathrm{H}_{8} \mathrm{O}_{7}$ (Merck) was dissolved into the solvent ethanol $\mathrm{C}_{2} \mathrm{H}_{5} \mathrm{OH}$ (Sigma-Aldrich) and also a stoichiometric amount of $\mathrm{Ti}(\mathrm{OBu})_{4}$ was added into maintaining the stirring vigorously. The molar quantity of citric acid was 2.5 times the mol of Gd and Ti that is $n_{\mathrm{CA}} /\left(n_{\mathrm{Gd}}+n_{\mathrm{Ti}}\right)=2.5$. On the other side, a stoichiometric amount of powder $\mathrm{Gd}_{2} \mathrm{O}_{3}$ was dissolved into a non-stoichiometric amount of concentrated nitric acid $\mathrm{HNO}_{3}\left(65 \%\right.$, Sigma-Aldrich) with some drops of water in order to obtain a $\mathrm{Gd}^{3+}$ dissolution of a molar rate $\mathrm{Gd} / \mathrm{Ti}=1$. This dissolution of $\mathrm{Gd}^{3+}$ was placed in magnetic stirring and the excessive $\mathrm{HNO}_{3}$ was evaporated by slow heating at $80^{\circ} \mathrm{C}$. Next, the dissolution containing Ti was poured into the $\mathrm{Gd}$ dissolution and this mixture was placed in an $80^{\circ} \mathrm{C}$ oil bath to vaporize water and excessive solvent. Here, the alkoxide $\left(\mathrm{Ti}(\mathrm{OBu})_{4}\right)$ is hydrolysed and forms hydroxyl $\left(\mathrm{Ti}(\mathrm{OBu})_{3}-\mathrm{OH}\right)$ and alkoxi $(\mathrm{Bu}-\mathrm{OH})$ where the former with the citric acid as a chelating agent results in a dissolution of $\mathrm{Ti}^{4+}$ [68]. Thus, we have a mixture of two dissolutions one containing $\mathrm{Gd}^{3+}$ and the other one $\mathrm{Ti}^{4+}$ with excessive organic compounds. The magnetic stirring was maintained for 12 hours until the mixture became highly viscous and finally changed into a yellow gel. Then, in order to remove residues of the organic compounds, this yellow gel of $\mathrm{Gd}_{2} \mathrm{Ti}_{2} \mathrm{O}_{7}$ was heat-treated in "air"; that is without any gaseous atmosphere; at $900^{\circ} \mathrm{C}$ for 3 hours. From that, the resulting polycrystalline powder of $\mathrm{Gd}_{2} \mathrm{Ti}_{2} \mathrm{O}_{7}$ is an extremely white one. This white powder was heat-treated once more at $1100^{\circ} \mathrm{C}$ for 24 hours in air and the resulting final powder of $\mathrm{Gd}_{2} \mathrm{Ti}_{2} \mathrm{O}_{7}$ is a darker one. This sample is referred to in this work as the "as-grown sample" and it is the starting point of our study presented in Chapter 4 in which different post-growth annealing conditions take place. To confirm that the phase of the polycrystalline powder is indeed $\mathrm{Gd}_{2} \mathrm{Ti}_{2} \mathrm{O}_{7}, \mathrm{X}$-ray powder diffraction was performed. X-ray powder diffraction is detailed in Section 3.2 .

The samples of $\mathrm{Gd}_{2} \mathrm{Ti}_{2} \mathrm{O}_{7}$ studied in Chapter 4 are separated in two different batches. The first set of $\mathrm{Gd}_{2} \mathrm{Ti}_{2} \mathrm{O}_{7}$ samples consists of a polycrystalline powder as-grown in air and two samples annealed in oxygen $\mathrm{O}_{2}$ for $24 \mathrm{~h}$ and $27 \mathrm{~h}$. That means that our as-grown polycrystalline sample of $\mathrm{Gd}_{2} \mathrm{Ti}_{2} \mathrm{O}_{7}$ will be annealed at $1100^{\circ} \mathrm{C}$ in $\mathrm{O}_{2}$ at a flow rate of $50 \mathrm{ml} \mathrm{min}^{-1}$ for $24 \mathrm{~h}$ and $27 \mathrm{~h}$. The second one consists of polycrystalline powder $\mathrm{Gd}_{2} \mathrm{Ti}_{2} \mathrm{O}_{7}$ grown in three different atmospheres: air, $\mathrm{H}_{2}$, and $\mathrm{O}_{2}$. Here, the polycrystalline $\mathrm{Gd}_{2} \mathrm{Ti}_{2} \mathrm{O}_{7}$ is heat-treated at $1100^{\circ} \mathrm{C}$ for 24 hours in air, in $\mathrm{H}_{2}$ at a flow rate of $50 \mathrm{ml} \mathrm{min}^{-1}$ and in $\mathrm{O}_{2}$ at a flow rate of $50 \mathrm{ml} \mathrm{min}^{-1}$. In order to measure the magnetic and 
thermal properties of $\mathrm{Gd}_{2} \mathrm{Ti}_{2} \mathrm{O}_{7}$, we compact the polycrystalline powder samples into circular pellets of $10 \mathrm{~mm}$ of diameter.

\subsubsection{Polycrystalline growth of $\mathrm{Gd}_{2-x} \mathrm{Y}_{x} \mathrm{Ti}_{2} \mathrm{O}_{7}$}

To prepare the polycrystalline powder of $\mathrm{Gd}_{2-x} \mathrm{Y}_{x} \mathrm{Ti}_{2} \mathrm{O}_{7}$ we employed the sol-gel method described above with extra steps. We used as starting materials: gadolinium oxide powder $\mathrm{Gd}_{2} \mathrm{O}_{3}(99.99 \%$, Reacton), tetrabutyl titanate $\mathrm{Ti}(\mathrm{OBu})_{4}$ (Gelest), and yttrium oxide powder $\mathrm{Y}_{2} \mathrm{O}_{3}$ (99.99\%, SigmaAldrich) which contains the non-magnetic ion $\mathrm{Y}^{3+}$ responsible for the dilution of the magnetic lattice. The stoichiometry of the chemical reaction is given by

$$
\left(1-\frac{x}{2}\right) \mathrm{Gd}_{2} \mathrm{O}_{3}+\frac{x}{2} \mathrm{Y}_{2} \mathrm{O}_{3}+2 \mathrm{TiO}_{2} \rightarrow \mathrm{Gd}_{2-x} \mathrm{Y}_{x} \mathrm{Ti}_{2} \mathrm{O}_{7} .
$$

Similar to the method described above, first an proper amount of powder citric acid $\mathrm{C}_{6} \mathrm{H}_{8} \mathrm{O}_{7}$ was dissolved into ethanol $\mathrm{C}_{2} \mathrm{H}_{5} \mathrm{OH}$ and a stoichiometric amount of $\mathrm{Ti}(\mathrm{OBu})_{4}$ was added to the dissolution maintaining the stirring. The molar quantity of citric acid was 2.5 times the mol of $\mathrm{Gd}$, Ti, and $\mathrm{Y}$ that is $n_{\mathrm{CA}} /\left(n_{\mathrm{Gd}}+n_{\mathrm{Y}}+n_{\mathrm{Ti}}\right)=2.5$. Then, concentrated $\mathrm{HNO}_{3}$ was used as a solvent of the oxides $\mathrm{Gd}_{2} \mathrm{O}_{3}$ and $\mathrm{Y}_{2} \mathrm{O}_{3}$ with some drops of water to obtain a dissolution of $\mathrm{Gd}^{3+}$ and $\mathrm{Y}^{3+}$ with a molar rate $\mathrm{Gd}+\mathrm{Y} / \mathrm{Ti}=1$. To evaporate the excessive $\mathrm{HNO}_{3}$ the solution was placed in magnetic stirring and heating. After that, the two solutions-one of $\mathrm{Ti}$ and the other one containing Gd and $\mathrm{Y}$-were mixed and placed in an $80^{\circ} \mathrm{C}$ oil bath to vaporize water and excessive solvent. The magnetic stirring was maintained for 12 hours until the mixture became into a gel. This gel of $\mathrm{Gd}_{2-x} \mathrm{Y}_{x} \mathrm{Ti}_{2} \mathrm{O}_{7}$ was heated in air at $900^{\circ} \mathrm{C}$ for 3 hours to evaporate excessive organic compounds. Next, the resulting powder of $\mathrm{Gd}_{2-x} \mathrm{Y}_{x} \mathrm{Ti}_{2} \mathrm{O}_{7}$ was heat-treated at $1100^{\circ} \mathrm{C}$ for 24 hours in air. A final heating at $1100^{\circ} \mathrm{C}$ for 24 hours in $\mathrm{O}_{2}$ at a flow rate of $50 \mathrm{ml} \mathrm{min}{ }^{-1}$ is made to obtain stoichiometric samples of $\mathrm{Gd}_{2-x} \mathrm{Y}_{x} \mathrm{Ti}_{2} \mathrm{O}_{7}$. We analysed the polycrystalline $\mathrm{Gd}_{2-x} \mathrm{Y}_{x} \mathrm{Ti}_{2} \mathrm{O}_{7}$ by $\mathrm{X}$-ray powder diffraction to verify the phase of our sample and to compare its X-ray diffractogram with the polycrystalline $\mathrm{Gd}_{2} \mathrm{Ti}_{2} \mathrm{O}_{7}$ of the previous subsection.

In Chapter 5 it is studied the polycrystalline $\mathrm{Gd}_{2-x} \mathrm{Y}_{x} \mathrm{Ti}_{2} \mathrm{O}_{7}$ samples with different concentrations of yttrium that is for $\mathrm{x}=0.1,0.14,0.16,0.2,0.3,0.4,0.7,1,1.2$, and 1.5 . Also, the polycrystalline powder samples of $\mathrm{Gd}_{2-x} \mathrm{Y}_{x} \mathrm{Ti}_{2} \mathrm{O}_{7}$ were compacted into circular pellets of $10 \mathrm{~mm}$ of diameter to measure their low-temperature magnetic properties. 


\section{2 $\mathrm{X}$-ray powder diffraction}

$\mathrm{X}$-ray diffraction (XRD) is a powerful technique to characterize the homogeneity, crystal structure, stoichiometry and phase of a sample. It occurs when the X-rays are scattered by the periodic array of the atoms in the solid producing constructive interference at specific angles. The wavelength of the $\mathrm{X}$-rays are similar to the distance between atoms being thus an excellent probe of the microscopic structure [10]. The scattering of the $\mathrm{X}$-rays from atoms produces a diffraction pattern when they satisfy the Bragg condition [10]:

$$
\lambda=2 d_{h k l} \sin \theta .
$$

It calculates the angle where constructive interference from X-rays scattered by parallel planes of atoms will produce a diffraction peak [10]. Here, the X-ray wavelength $\lambda$ is fixed and thus a family of planes produces a diffraction peak only at a specific angle $\theta . d_{h k l}$ is the distance between parallel planes of atoms in the family (hkl) and it is a geometric function of the size and shape of the unit cell $[10]$.

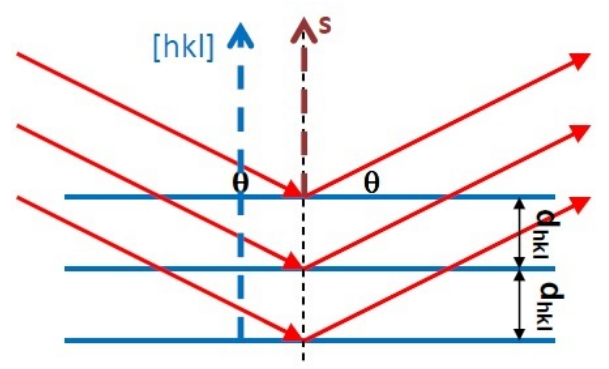

Figure 3.1: Diagram of the diffractometer. This figure was taken from [69].

Bragg's condition provides a simple model to understand what conditions are required for diffraction as shown in figure 3.1 from [69]. For parallel planes of atoms, with a space $d_{h k l}$ between the planes, constructive interference only occurs when Bragg's condition is satisfied. The plane normal $[h k l]$ must be parallel to the diffraction vector $\mathbf{s}[69]$. Here, the plane normal $[h k l]$ is the direction perpendicular to a plane of atoms and the diffraction vector $\mathbf{s}$ is the vector that bisects the angle between the incident and diffracted beam [69].

The diffraction technique used in this dissertation to determine the phase and quality of the samples is powder $\mathrm{X}$-ray diffraction. Here, the sample is crushed into a polycrystalline powder. A single wavelength of incident $\mathrm{X}$-rays is used, and the $2 \theta$ angle is varied. As the sample was ground 
into a powder, the diffraction pattern is averaged over all crystallographic directions. A diagram of the diffractometer is shown in figure 3.2 from [69]. It illustrates the $\mathrm{X}$-ray tube as the source of the beam and the detector of the diffracted X-rays. Figure 3.2 also shows the Bragg-Brentano geometry of the diffractometer in which the incident and diffracted beams are focussed at a fixed radius from the sample position [69]. The diffraction angle, $2 \theta$, is defined between the incident beam and the detector. The incident angle, $\omega$, is defined between the X-ray source and the sample, and it is the half of the diffraction angle $2 \theta$.

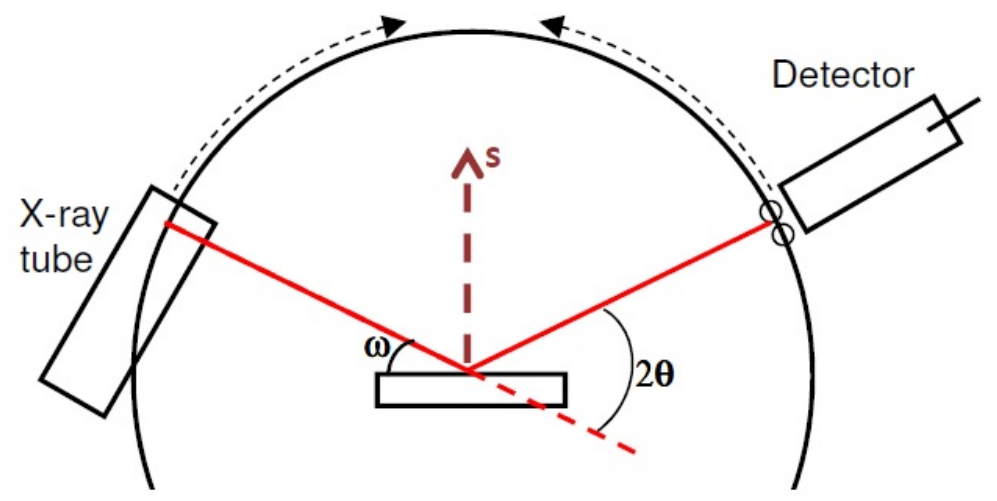

Figure 3.2: Diagram of the diffractometer. The diffraction angle, $2 \theta$, and the incident angle, $\omega$, which is the half of the diffraction angle $2 \theta$. Figure from [69].

For the powder $\mathrm{X}$-ray diffraction of the samples of $\mathrm{Gd}_{2} \mathrm{Ti}_{2} \mathrm{O}_{7}$ and $\mathrm{Gd}_{2-x} \mathrm{Y}_{x} \mathrm{Ti}_{2} \mathrm{O}_{7}$ it was used the diffractometer -Shimadzu XRD-7000- from the group of Dr. Flavio Vichi at the Institute of Chemistry of the University of São Paulo. Bragg-Brentano geometry and Cu K $\alpha 1$ radiation (1.5406 $\AA$ ) was used. $\mathrm{Gd}_{2} \mathrm{Ti}_{2} \mathrm{O}_{7}$ and $\mathrm{Gd}_{2-x} \mathrm{Y}_{x} \mathrm{Ti}_{2} \mathrm{O}_{7}$ samples were pulverized into fine powder to run the diffraction experiments. In Chapter 4 and 5 we present the $\mathrm{X}$-ray diffraction patterns of our samples and their respective structural analysis by the Rietveld method. The Rietveld refinement, which is detailed in [72], is an excellent method to validate the structure and the space group in which the material belongs [70]. Then, we use the software FullProf [71] with implemented Rietveld method to refine the collected data. 


\subsection{Magnetic measurements}

\subsubsection{Vibrating Sample Magnetometer (VSM)}

The principle of operating of the Vibrating Sample Magnetometer (VSM) is based in the Faraday's law of induction in which an induced electromotive force arises in a closed loop when there is a timevariation of the magnetic flux through the loop. The time-dependent induced voltage is given by the following equation

$$
V(t)=\sum_{n} \int_{\boldsymbol{S}} \frac{\partial \boldsymbol{B}(t)}{\partial t} \cdot d \boldsymbol{S},
$$

where $n$ is the number of loops in the pickup coils and $S$ its respective area. Using the VSM it was collected magnetization saturation data as a function of the applied magnetic field at fixed temperatures.

(a)

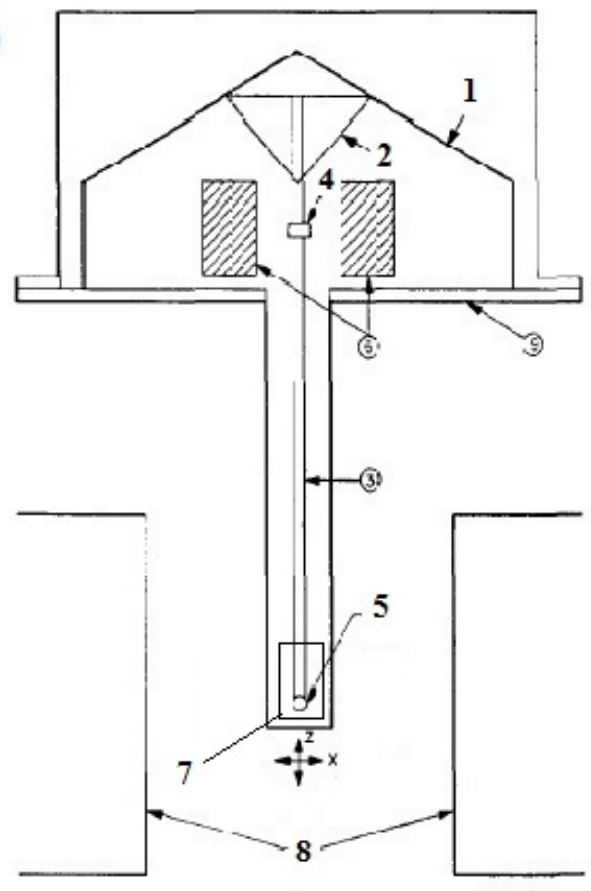

(b)

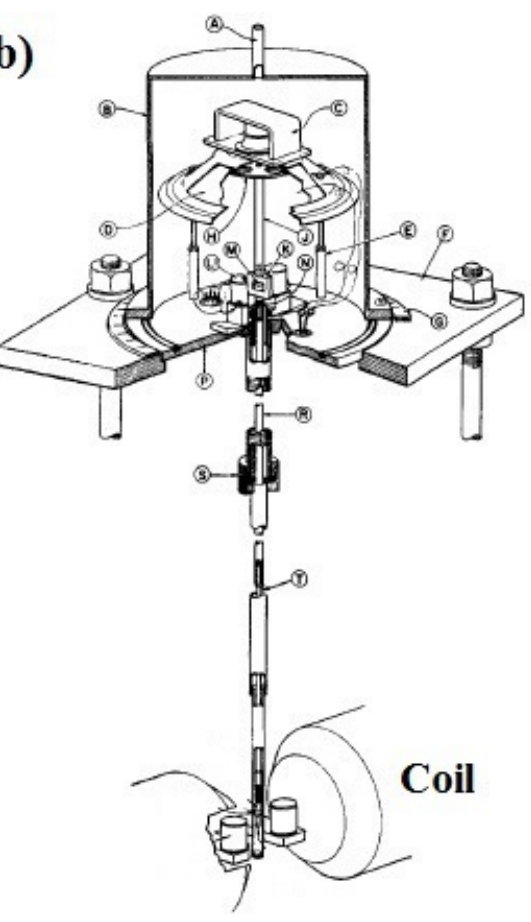

Figure 3.3: (a) Diagram of the vibrating sample magnetometer (VSM). (b) Detailed mechanical features of the VSM. This figure was adapted from [73].

All the magnetic induction measurements involve observation of the voltage induced in a pickup coil by a flux change when the applied magnetic field, coil position, or sample position is changed. A 
simplified diagram of the VSM is shown in figure 3.3(a) from [73]. Here, the applied magnetic field is generated by the superconducting coil (8). The sample (5) vibrates parallel to the applied field by the assembly (1), (2), and (4) [73]. Then, the oscillating magnetic field of the vibrating sample induces a voltage in the stationary pickup coils (7), and from measurements of this voltage, which is proportional to the magnetic moment, it is determined the magnetic moment of the sample [73]. This technique of measurement is capable of an extremely high sensitivity in which changes of the order of $10^{-5}$ to $10^{-6}$ emu are detected [73]. Figure 3.3(b) from [73] shows a more detailed view of the VSM. It shows the coil source of the applied magnetic field and also the sample centrally positioned between them.

Magnetization measurements in the VSM magnetometer are examples of dc or direct measurements. On them, the magnetization, $M$, does not change with time and it is measured for some applied field, $H$. As we mentioned above, the periodic movement of the sample gives rise to a variation of the magnetic flux and consequently to an output induced signal. This induced voltage detected by the pickup coils, $V$, depends of the magnetic moment of the sample $\sigma$, the frequency of the assembly $f$, the amplitude of vibration $A$, and $G$ a factor that depends of the geometry of the pickup coils:

$$
V=\sigma G A 2 \pi f \cos (2 \pi f t)
$$

The magnetization measurements using the VSM were carried out at a pumped helium-4 cryostat as shown in figure 3.4. This system reaches a temperature of $1.2 \mathrm{~K}$ with vacuum pumping and possesses a superconducting coil able to generate a magnetic field of $20 \mathrm{~T}$. To operate the pumped helium-4 cryostat with the VSM, first, it is required to cool down the space of the superconducting coil (in cyan) with liquid nitrogen $(77 \mathrm{~K})$. After the system reaches the thermal equilibrium, the liquid nitrogen is pumped out and the helium $(4.2 \mathrm{~K})$ is transferred into the superconducting coil space. Then, to cool down the sample space (in red), it is required to transferred helium-4 to the anti-dewar (in blue) allowing the thermal contact with the inner chamber of the sample space. By pumping the anti-dewar, the gas of helium-4 of the sample is liquefied. To reach temperatures down to $4.2 \mathrm{~K}$, it is used a vacuum pump in the sample space allowing a minimum temperature of $1.2 \mathrm{~K}$. Here in the inner chamber, it is also used liquid helium-3 to reach temperatures down to $1.26 \mathrm{~K}$, and using a vacuum pump it reaches a minimum temperature of $0.4 \mathrm{~K}$. 


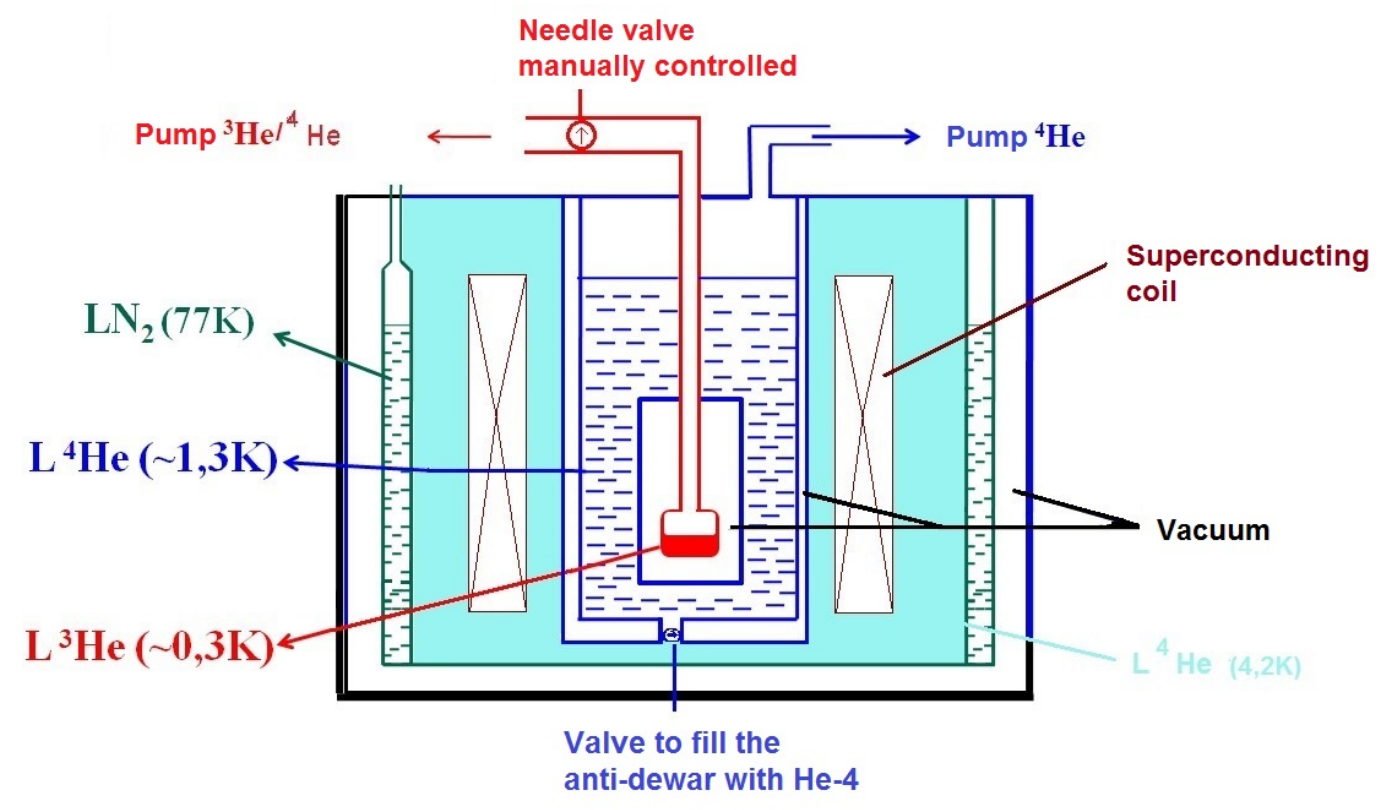

Figure 3.4: Schematic diagram of the VSM inside the pumped helium-4 cryostat.

\subsubsection{Home-made AC susceptometer}

The measurements of the ac or dynamic magnetic susceptibility were carried out at a home-made AC susceptometer. It consists of a pumped helium-4 cryostat; analogue to the figure 3.4 of the VSM; and of a mutual inductance bridge. The AC susceptometer reaches a temperature of $1.1 \mathrm{~K}$ using liquid helium-4 and a minimum one of $0.35 \mathrm{~K}$ using liquid helium-3. Also, it consists of a superconducting coil able to generate a magnetic field up to 7.5 T. As in the VSM magnetometer, the AC susceptometer uses pickup coils to detect changes in the magnetic flux due to the sample.

In an $\mathrm{AC}$ susceptometer, an ac field $h_{a c}$ is produced by a primary coil which is applied to the sample. This $h_{a c}$ can be applied to the sample in addition to a static field $H$. The total field is given by

$$
H_{T}=H+h_{a c},
$$

where $h_{a c}=h_{0} \cos (\omega t)$. Then, the ac magnetic susceptibility $\chi$ is given by [74]

$$
\chi=\frac{d M}{d h_{a c}},
$$


where $M$ is the magnetization of the sample.

Thus, the magnetic susceptibility $\chi$ can be studied as a complex and having two components: $\chi^{\prime}+i \chi^{\prime \prime}$. Indeed, on our measurements we focus on the real component $\chi^{\prime}$ that represents the component of the susceptibility that is in phase with the applied ac field. Because of that, we choose an appropriate phase to suppress the imaginary component $\chi^{\prime \prime}$ which is related to the energy losses.

In the operating of the $\mathrm{AC}$ susceptometer, it is used together with the cryostat a mutual inductance bridge. The sample is placed between two coupled coils known as the primary and secondary coils. This configuration is shown in figure 3.5. The secondary coils are two identical pickup coils positioned symmetrically inside the primary coil and are connected in opposition in order to cancel the voltages induced by the ac field itself or external sources. Thus, the sample susceptibility is determined from the difference between the values of inductance of the bridge with the sample in the secondary coil and without the sample.

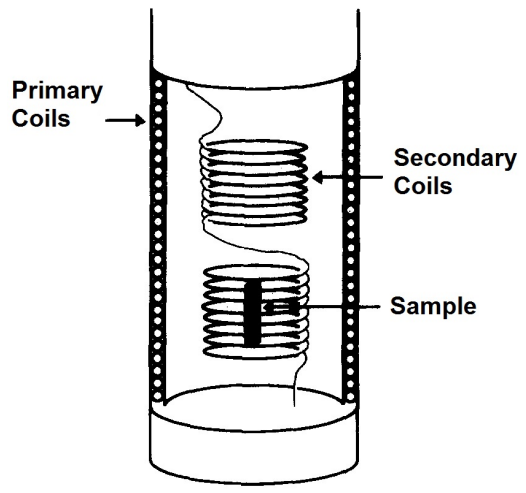

Figure 3.5: Primary and secondary coil configuration. Figure taken from [74].

To collect the data in the ac susceptometer, it was used a typical setup as detailed in reference [74]. Measuring the induced voltage with a lock amplifier, the real, $\chi^{\prime}$, and the imaginary, $\chi^{\prime \prime}$, parts of the susceptibility can be separated. An ac signal produced by the primary coil is applied to the sample and then the sample induce a signal on the secondary coils. Thus, this induced signal proportional to the ac magnetic susceptibility $\chi$ is measured. 


\subsubsection{Superconducting Quantum Interference Device (SQUID)}

The Superconducting Quantum Interference Device (SQUID) is the most sensitive measuring instrument to probe extremely low magnetic fields up to a magnitude order of $10^{-14} \mathrm{~T}$ [75]. The SQUID is a superconducting device that measure magnetic flux and output voltage signal. From this output voltage, it is determined the magnetic moment of a material and consequently its magnetization and magnetic susceptibility. In fact, the SQUID consists of a superconducting ring with two Josephson junctions (denoted $\mathrm{X})$ as is shown in figure 3.6. A Josephson Junction is a non-superconducting material (usually an insulator) sandwiched between two superconductors, forming a weak link. To understand how a Josephson junction works, we will discuss briefly superconductivity, the quantization of the magnetic flux, and the Josephson effect.

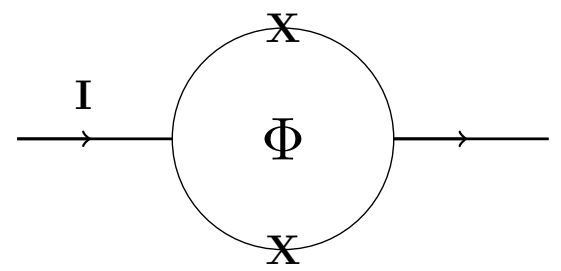

Figure 3.6: Diagram showing the Josephson junctions (denoted X), and the superconducting ring. $\Phi$ is the magnetic flux through the superconducting ring and $\mathrm{I}$ is the current through the loop.

Superconductivity is a remarkable phenomenon concerning the ability of certain materials to pass electrical currents [10]. It occurs when they are cooled down to a sufficiently low temperature that their electrical resistance vanishes completely [10]. This phenomenon is tied to the quantum-mechanical nature of solids, specifically, to the tendency of the electrons to form organized collective quantum mechanical states [76]. One instance of this are the Cooper pairs of electrons that behave cooperatively and form a single quantum-mechanical state [76]. In normal conductors, the resistance to the electrical current is caused by the electrons scattering off of the vibrating atomic nuclei (phonons), this scattering changes the electron's direction and transfers energy from the electrical current to the sound waves. In superconductors the electrons also interact with the phonons, but instead of scattering off of them and diminishing the flow of electrical current, the phonons actually promote the formation and movement of Cooper pairs [77]. It is because the phonons create a local concentration of positive electrical charge as the sound wave move past them [77].

Also, superconductivity provides a unique opportunity to observe the quantization of a physical 
quantity as the magnetic flux in a macroscopic system. To discuss the quantization of the magnetic flux, we first consider the magnetic flux, $\Phi$, inside a superconducting ring as is shown in figure 3.6. If the flux through the ring changes, circulating currents of Cooper pairs will arise in the ring to cancel the change. These circulating currents can be detected using a phenomenon called the Josephson effect, and hence, the small magnetic field can be measured. The answer to the quantization of the magnetic flux lies in the long-range coherence of the superconducting wavefunction $\Psi$. In addition, the geometry of a superconducting ring places special restrictions on the superconducting wavefunction. In order for the wavefunction to have a single value at a given point in the superconducting ring, the wavefunction must satisfy $\Psi(0)=\Psi(2 \pi)$. From this condition, it is derived that the amount of magnetic flux $\Phi$ contained within in the ring can only assume certain discrete values of the flux quantum $\phi_{0}=h / 2 e[77]$ :

$$
\Phi=n \phi_{0}=\frac{n h}{2 e}
$$

where $n$ is an integer, $h$ is the Planck's constant, and $e$ the fundamental charge of the electron.

As we mentioned before, the superconducting ring in the SQUID device presents two Josephson junctions. These junctions are thin gaps that separate two superconducting regions and in which occurs the Josephson effect. This phenomenon allows to detect circulating currents and thus it is the basis of the SQUID operation. First, we suppose that we have in the two superconducting regions two wavefunctions of the Cooper pairs with different phases $\varphi_{1}$ and $\varphi_{2}$. So, in the thin insulator gap takes place an overlapping of the wavefunctions which reduce their amplitude without being vanished. This is due to the thin gap between two superconducting regions allows the tunneling of Cooper pairs of electrons through it. Thus, in the Josephson effect a supercurrent, $I_{s}$, appears inside the insulator without dissipation or any resistance that depends of the phase change $\Delta \phi=\varphi_{1}-\varphi_{2}$ [77]:

$$
I_{s}=I_{c} \sin (\Delta \phi)
$$

where $I_{c}$ is the critical or maximum supercurrent that the junction can support. The voltage between the two junctions is a periodic function of the magnetic flux inside the loop [77]. The change in the magnetic flux $\Phi$ through the ring generates a measurable change in the current through the Josephson junction [77]. Thus, the voltage is given by [77]:

$$
V=\frac{R}{2} \sqrt{I^{2}-\left[2 I_{c} \cos \left(\frac{\pi \Phi}{\phi_{0}}\right)\right]^{2}} .
$$


Here $R$ is the resistance of the Josephson junction, $I$ the current trough the superconducting ring, and $I_{c}$ is the critical current.

A diagram of the SQUID detection system is shown in figure 3.7. On the left side, it is shown the pickup coils to detect the signal and the sample. Here, the sample is positioned in the center of two pickup coils and a magnetic field is applied in a stablish direction. This magnetic field generates an induced current in the pickup coils that goes through the loop which is inductively coupled to the SQUID device. On the right side of figure 3.7, it is shown the SQUID device as presented in figure 3.6 which works as we discussed above.

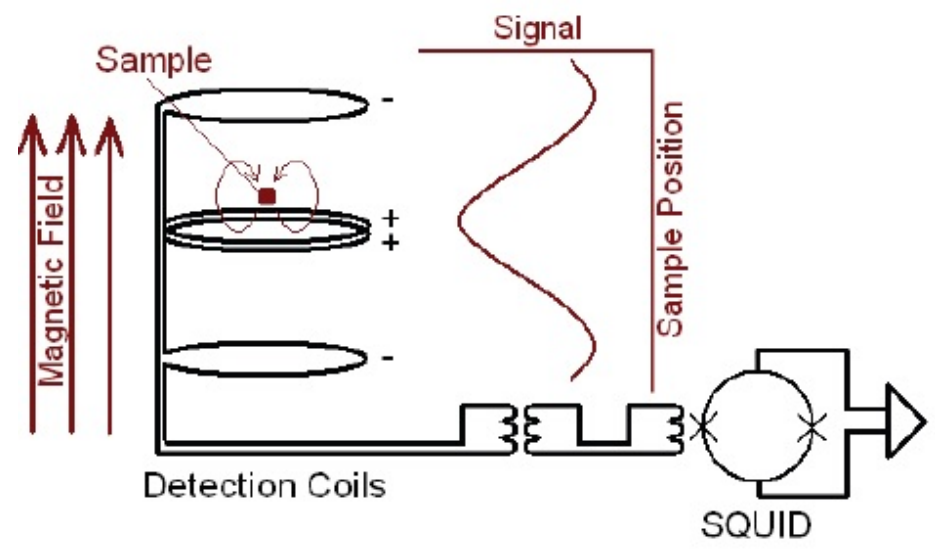

Figure 3.7: Diagram of detection of the SQUID. This figure was adapted from [75].

To measure magnetization and ac susceptibility it was used a SQUID -Model MPMS XL-7- of the Quantum Design, operating at a cryostat of ${ }^{4} \mathrm{He}$ to reach temperatures in the range $2 \mathrm{~K}$ to $400 \mathrm{~K}$ and magnetic fields up to $7 \mathrm{~T}$. In order to reduce the effects of demagnetization in our measurements, the circular pellets of $\mathrm{Gd}_{2} \mathrm{Ti}_{2} \mathrm{O}_{7}$ and $\mathrm{Gd}_{2-x} \mathrm{Y}_{x} \mathrm{Ti}_{2} \mathrm{O}_{7}$ were cut into needle shapes. 


\subsection{Calorimetric measurements}

\subsubsection{Physical Property Measurement System (PPMS)}

To collect the specific-heat data we used the calorimeter Physical Property Measurement System (PPMS) in heat capacity option of the Quantum Design. It measures the specific heat or heat capacity of a material which is defined by the amount of energy required to raise the temperature by a unit amount:

$$
C_{p}=\left(\frac{d Q}{d T}\right)_{p},
$$

where $d Q$ is the applied heat, and $d T$ is the resultant change in temperature of the sample.

In the PPMS during a measurement, a known amount of heat is applied at constant power for a fixed time, and then the heating period is followed by a cooling period of the same duration [78]. As is shown in figure 3.8 from [78], the PPMS consists of a platform heater and platform thermometer which are attached to the bottom side of the sample platform. Figure 3.8 also shows the small wires that provide electrical connection to the platform heater and platform thermometer as well as the thermal connection and support for the platform [78]. The sample is mounted to the platform by using a thin layer of vacuum grease, which provides the required thermal contact to the platform [78].

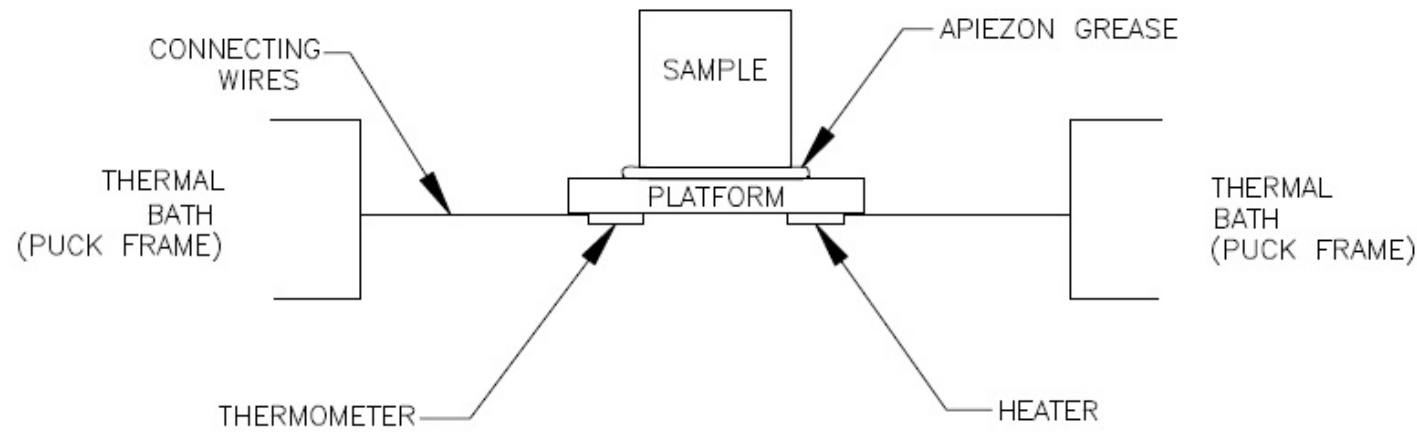

Figure 3.8: Sample and sample platform in the PPMS. This figure was taken from [78].

The measurement technique of the PPMS consists of a relaxation technique in which after each measurement cycle it fits the temperature response of the sample platform to a model that accounts for both the thermal relaxation of the sample platform to the bath temperature and the relaxation between the sample platform and the sample itself [78]. In a simple model, the temperature $T$ of the 
platform as a function of time $t$ obeys the equation [78]

$$
C \frac{d T}{d t}=-K_{\mathrm{w}}\left(T-T_{b}\right)+P(t)
$$

where $C$ is the total specific heat of the sample and sample platform, $K_{w}$ is the thermal conductance of the supporting wires, $T_{b}$ is the temperature of the thermal bath, and $P(t)$ is the power applied by the heater. The heater power $P(t)$ is equal to $P_{0}$ during the heating period of the measurement and is reduced to zero during the cooling period [78]. The solution of this equation is given by an exponential function with a characteristic time constant $\tau$ equal to $C / K_{\mathrm{w}}$ [78]. Thus, the time-dependence (or temperature dependence) of the specific heat is given by [78]

$$
C(t)=K_{\mathrm{w}} \Delta t / \ln \left(\frac{T(t)-T_{b}-P}{T(t+\Delta t)-T_{b}-P}\right) .
$$

In order to collect the specific heat data of the sample we first measure the specific heat of the addenda (platform and Apiezon grease) $C_{a d d}$. Then, we put the sample on the addenda and measure again the total specific heat $C$. Finally, we subtract $C-C_{a d d}$ to obtain the specific heat of the sample. 


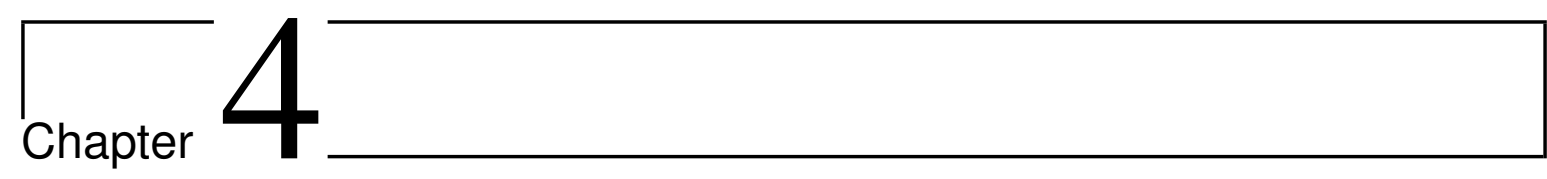

\section{Vacancy Defects in Oxygen-deficient}

\section{$\mathrm{Gd}_{2} \mathrm{Ti}_{2} \mathrm{O}_{7}$}

In this chapter we discuss the effects of the vacancy defects in the structural, magnetic, and thermal properties of the pyrochlore $\mathrm{Gd}_{2} \mathrm{Ti}_{2} \mathrm{O}_{7}$. We study the pyrochlore $\mathrm{Gd}_{2} \mathrm{Ti}_{2} \mathrm{O}_{7}$ with vacancy defects near to the temperature of its first phase transition $\mathrm{T}_{\mathrm{N}}^{1} \sim 1 \mathrm{~K}$ in two different batches of polycrystalline $\mathrm{Gd}_{2} \mathrm{Ti}_{2} \mathrm{O}_{7}$. The first batch consists of $\mathrm{Gd}_{2} \mathrm{Ti}_{2} \mathrm{O}_{7}$ samples with post-growth annealings in oxygen and the second one consists of $\mathrm{Gd}_{2} \mathrm{Ti}_{2} \mathrm{O}_{7}$ samples grown in different atmospheres: air, oxygen, and hydrogen. Actually, in all these $\mathrm{Gd}_{2} \mathrm{Ti}_{2} \mathrm{O}_{7}$ samples it is expected to obtain non-stoichiometric $\mathrm{Gd}_{2} \mathrm{Ti}_{2} \mathrm{O}_{7-\delta}$ compositions which present a small degree of deficiency of oxygen. To quantify the vacancy defects of the pyrochlore $\mathrm{Gd}_{2} \mathrm{Ti}_{2} \mathrm{O}_{7}$, it was refined the calculated X-ray diffraction data by the Rietveld method using the software FullProf. Also, in order to discuss the low-temperature ground state of our $\mathrm{Gd}_{2} \mathrm{Ti}_{2} \mathrm{O}_{7}$ samples, we report experimental data of ac magnetic susceptibility, magnetization, and specific heat. 


\subsection{Results of $\mathrm{Gd}_{2} \mathrm{Ti}_{2} \mathrm{O}_{7}$ annealed in oxygen}

The aim of this section is to discuss the effect of the oxygen annealing in $\mathrm{Gd}_{2} \mathrm{Ti}_{2} \mathrm{O}_{7}$ on its magnetic properties. The polycrystalline $\mathrm{Gd}_{2} \mathrm{Ti}_{2} \mathrm{O}_{7}$ samples were prepared with the sol gel method described in Chapter 3. Specifically, this first batch consist of three samples: one as-grown sample of $\mathrm{Gd}_{2} \mathrm{Ti}_{2} \mathrm{O}_{7}$ and other two $\mathrm{Gd}_{2} \mathrm{Ti}_{2} \mathrm{O}_{7}$ samples annealed in oxygen. The first and the second annealing in oxygen were carried out at a rate of $50 \mathrm{ml} / \mathrm{min}$ for 24 hours and 27 hours respectively at $1100^{\circ} \mathrm{C}$. Then, using Rietveld refinement we discuss results for the structural parameters obtained by X-ray diffraction. Also, we study the magnetic properties of our three $\mathrm{Gd}_{2} \mathrm{Ti}_{2} \mathrm{O}_{7}$ samples by measurements of ac susceptiblity and magnetization.

\subsubsection{Structural analysis}

We present a structural study of the powder X-ray diffraction data for the sample of $\mathrm{Gd}_{2} \mathrm{Ti}_{2} \mathrm{O}_{7}$ with a second annealing in oxygen. We report results only for this sample because we have the assumption that a second annealing in oxygen leads to a sample with ideal stoichiometry. $\mathrm{X}$-ray diffraction data collected at room temperature is shown in figure 4.1 (red points). The diffraction peaks shown are consistent with the positions of the expected peaks (green lines) for the pyrochlore structure.

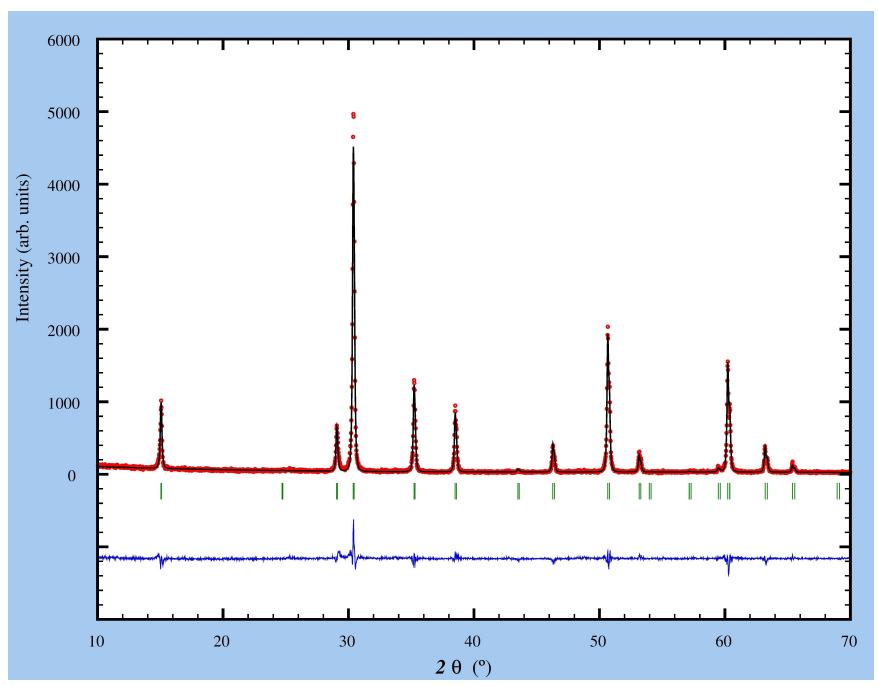

Figure 4.1: Powder X-ray diffraction (red points) and calculated (black solid line) data for $\mathrm{Gd}_{2} \mathrm{Ti}_{2} \mathrm{O}_{7}$ annealed in oxygen. The Rietveld refinement using Fullprof software was performed with the $\mathrm{X}$-ray diffraction pattern of $\mathrm{Gd}_{2} \mathrm{Ti}_{2} \mathrm{O}_{7}$ measured at room temperature. 
CHAPTER 4. Vacancy Defects in Oxygen-deficient $\mathrm{Gd}_{2} \mathrm{Ti}_{2} \mathrm{O}_{7}$

The structural analysis was done by using Rietveld refinement software FullProf and the graphical interface WinPLOTR. The calculated profile assuming a stoichiometric model of $\mathrm{Gd}_{2} \mathrm{Ti}_{2} \mathrm{O}_{7}$ is shown in figure 4.1 (black solid line). The curve in the lower side (blue) represents the difference between the data and the model. Results from the Rietveld analysis are shown in table 4.1. The refinement revealed that the $\mathrm{X}$-ray diffraction data refine in the pyrochlore structure with $F d \overline{3} m$ space group. As mentioned before, Gd and Ti ions are located on corner-sharing tetrahedra lattices, and there are two inequivalent oxygen sites: $\mathrm{O}(1)$ located at the centre of the Gd tetrahedra, and $\mathrm{O}(2)$ filling interstitial regions. Then, we found that the occupancies of the Gd and Ti sites are very close to one (a site without vacancies is ideally one) excluding the possibility of vacancies on these sites. The occupancy of the $\mathrm{O}(2)$ sites are also close to one, however the occupancy of the $\mathrm{O}(1)$ sites is much lower than one. Thus, the oxygen vacancies were found to be mainly on the $\mathrm{O}(1)$ sites. The value of chi-squared $\chi^{2}$ (a measure of the goodness of the fit which is ideally one) is 2.09 which indicates that the refinement could be still improved. However, this value of $\chi^{2}$ was the minimum obtained and as shown in figure 4.1 there are slight differences between the experimental and calculated data, so we keep this value. Furthermore, the lattice parameter for $\mathrm{Gd}_{2} \mathrm{Ti}_{2} \mathrm{O}_{7}$ annealed in oxygen, $10.184 \AA$, is consistent with the values reported by $[39,51]$.

\begin{tabular}{c|c}
\hline $\mathrm{Gd}_{2} \mathrm{Ti}_{2} \mathrm{O}_{7}$ & Second annealing \\
\hline \hline Space group & $F d \overline{3} m$ \\
\hline Lattice parameter & $10.184 \AA$ \\
\hline $\mathrm{Gd}$ & 1.03 \\
\hline $\mathrm{Ti}$ & 1.05 \\
\hline $\mathrm{O}(1)$ & 0.66 \\
\hline $\mathrm{O}(2)$ & 0.98 \\
\hline$\chi^{2}$ & 2.09 \\
\hline \hline
\end{tabular}

Table 4.1: Refinement of the structure of $\mathrm{Gd}_{2} \mathrm{Ti}_{2} \mathrm{O}_{7}$ annealed in oxygen showing the space group, the lattice parameter, the occupancies of each site, and the value of chi-squared. 


\subsubsection{AC magnetic susceptibility}

AC magnetic susceptibility measurements were conducted on needle-shaped samples for the as-grown, first annealing and second annealing in oxygen $\mathrm{Gd}_{2} \mathrm{Ti}_{2} \mathrm{O}_{7}$. Above $4.2 \mathrm{~K}$, the ac magnetic susceptibility $\chi_{a c}$ was measured using the SQUID. Below $4.2 \mathrm{~K}, \chi_{a c}$ was measured on the ac susceptometer using a mutual inductance bridge at modulation frequency $f=155 \mathrm{~Hz}$ and ac field $\mu_{0} h_{a c}=10 \mathrm{mT}$.

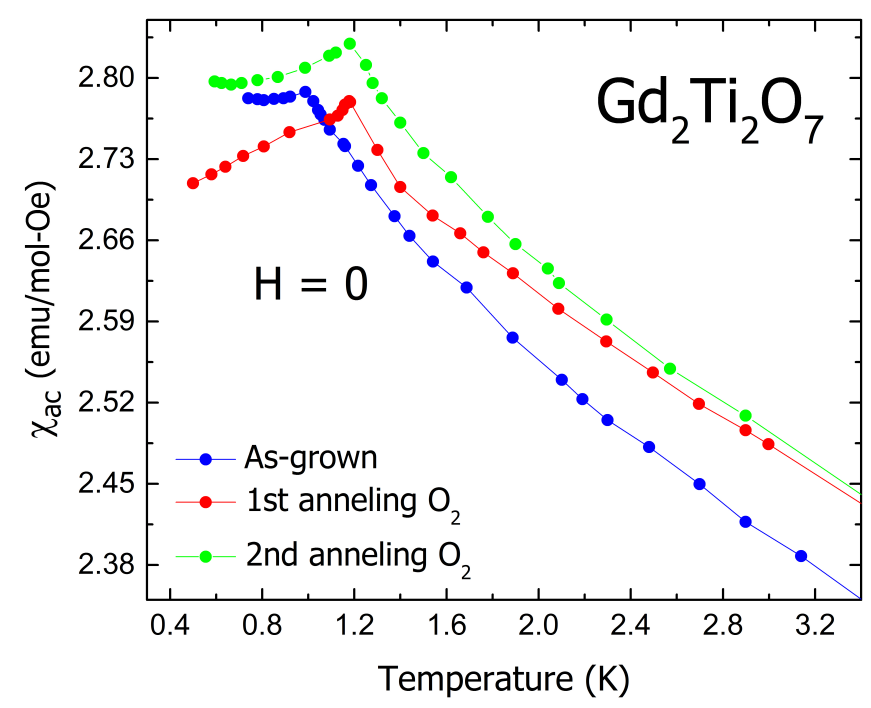

Figure 4.2: ac magnetic susceptibility $\chi_{a c}$ as a function of the temperature for the as-grown, first annealing and second annealing in oxygen $\mathrm{Gd}_{2} \mathrm{Ti}_{2} \mathrm{O}_{7}$ samples measured at zero field.

Figure 4.2 shows the ac magnetic susceptibility data for the as-grown $\mathrm{Gd}_{2} \mathrm{Ti}_{2} \mathrm{O}_{7}$ and also for the samples with a first and a second annealing in oxygen in the temperature range of 0.5 to $2.25 \mathrm{~K}$. For the three samples, it shows the expected peak of the first phase transition $\sim 1 \mathrm{~K}$ which corresponds to a partial ordering of the spins of $\mathrm{Gd}_{2} \mathrm{Ti}_{2} \mathrm{O}_{7}$. This phase transition is in agreement with previously reported results $[15,39]$. The values of susceptibility near to $1 \mathrm{~K}$ were difficult to measure since it changed rapidly but it was evident a sizeable increasing and a maxima at this temperature. After the phase transition, the magnetic susceptibility values start to decrease slowly. The phase transition of the samples with a first and second annealing in oxygen occurs almost at the same temperature $\mathrm{T}_{\mathrm{N}}^{1} ; 1.18 \mathrm{~K}$ and $1.17 \mathrm{~K}$ respectively; whereas the as-grown sample has its phase transition slightly at a lower temperature $\mathrm{T}_{\mathrm{N}}^{1}=1.01 \mathrm{~K}$. This could infer that an extra second annealing in oxygen makes no difference at all but figure 4.2 tells us that they do not have exactly the same behaviour at low 
temperatures. It also indicates that the as-grown sample which we think that possesses more vacancies of oxygen has a higher degree of frustration. We observe that the intensity of the peak of magnetic susceptibility increases as we anneal the samples in oxygen for an extended time. Furthermore, we notice that the $\mathrm{Gd}_{2} \mathrm{Ti}_{2} \mathrm{O}_{7}$ sample with a second annealing in oxygen (green) seems to develop a more defined order at $\mathrm{T}_{\mathrm{N}}^{1}=1.17 \mathrm{~K}$ which reflects on the intensity of the peak. This temperature is higher than the transition temperature of the as-grown sample $\left(\mathrm{T}_{\mathrm{N}}^{1}=1.01\right)$ meaning that there is a lower degree of geometric frustration in the sample with oxygen annealing. The variation of the temperature at the first phase transition for all our samples is correlated to the stoichiometry of the oxygen-deficient $\mathrm{Gd}_{2} \mathrm{Ti}_{2} \mathrm{O}_{7}$ samples.
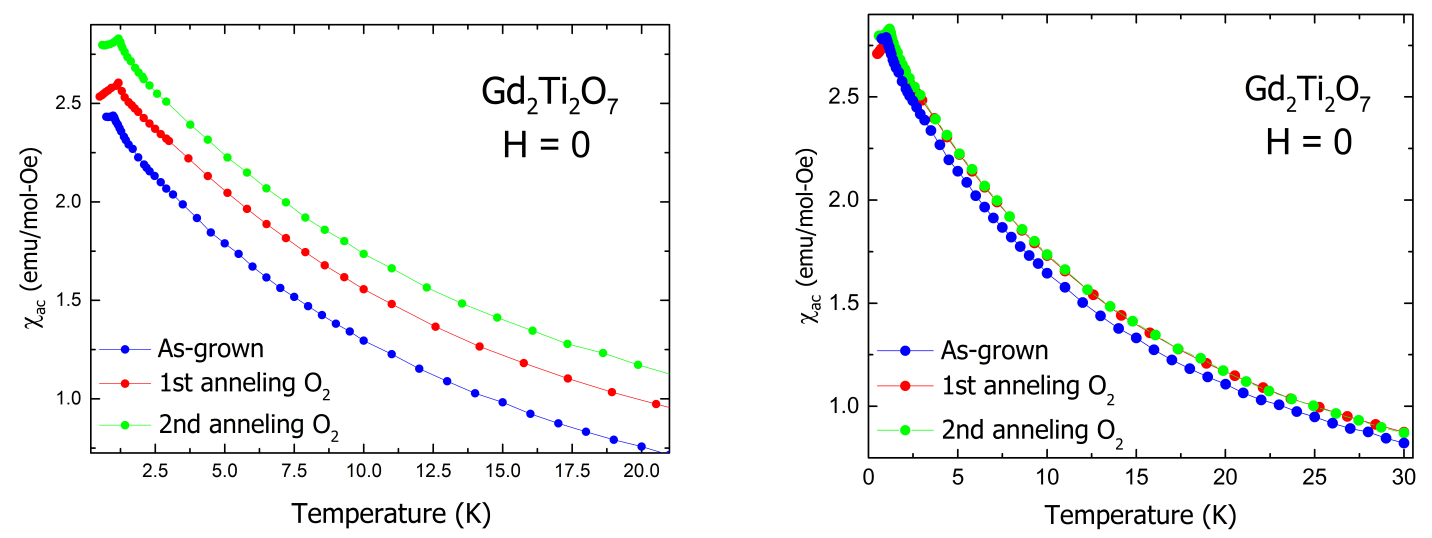

Figure 4.3: Left: Full $\chi_{a c}$ data between $1 \mathrm{~K}$ and $20 \mathrm{~K}$ for the as-grown, first annealing and second annealing in oxygen $\mathrm{Gd}_{2} \mathrm{Ti}_{2} \mathrm{O}_{7}$ samples at zero field. The $\chi_{a c}$ curves are slightly offset for clarity. Right: $\chi_{a c}$ data between 1 and $30 \mathrm{~K}$ without the offset.

The left side of figure 4.3 shows the full data of the ac magnetic susceptibility for the $\mathrm{Gd}_{2} \mathrm{Ti}_{2} \mathrm{O}_{7}$ samples. Here, for the as-grown sample of $\mathrm{Gd}_{2} \mathrm{Ti}_{2} \mathrm{O}_{7}$ (blue) there is almost no variation on the data after the peak of the phase transition. For the annealed samples in oxygen (red and green), each one of the magnetic susceptibility points after the magnetic transition were measured after a long time of relaxation waiting to stabilize the magnetic susceptibility value. The right side of figure 4.3 shows the raw data of the $\chi_{a c}$ for the $\mathrm{Gd}_{2} \mathrm{Ti}_{2} \mathrm{O}_{7}$ samples. In our experiments we were not able to resolve the second magnetic transition at $\mathrm{T}_{\mathrm{N}}^{2} \sim 0.75 \mathrm{~K}$ since small variations on the temperature seemed not to produce any difference on the magnetic susceptibility. The second phase transition at $0.75 \mathrm{~K}$ is visible as a lower peak but present in the $\chi_{a c}$ results of [60] for a $\mathrm{Gd}_{2} \mathrm{Ti}_{2} \mathrm{O}_{7}$ crystal so for our polycrystalline 
CHAPTER 4. Vacancy Defects in Oxygen-deficient $\mathrm{Gd}_{2} \mathrm{Ti}_{2} \mathrm{O}_{7}$

samples we did not expect to resolve this peak. From the previous $\chi_{a c}$ curves, we report the values of the ordering temperatures in table 4.2. The annealing in oxygen of $\mathrm{Gd}_{2} \mathrm{Ti}_{2} \mathrm{O}_{7}$ takes the phase transition to the same temperature as is shown by the almost identical values of $\mathrm{T}_{\mathrm{N}}^{1}: 1.18$ and $1.17 \mathrm{~K}$.

\begin{tabular}{c|c|c|c}
\hline $\mathrm{Gd}_{2} \mathrm{Ti}_{2} \mathrm{O}_{7}$ & As-grown & First annealing & Second annealing \\
\hline \hline Annealing in oxygen for & $0 \mathrm{~h}$ & $24 \mathrm{~h}$ & $27 \mathrm{~h}$ \\
\hline $\mathrm{T}_{\mathrm{N}}^{1}(\mathrm{~K})$ & 1.01 & 1.18 & 1.17 \\
\hline \hline
\end{tabular}

Table 4.2: Transition temperatures, $\mathrm{T}_{\mathrm{N}}^{1}$, for $\mathrm{Gd}_{2} \mathrm{Ti}_{2} \mathrm{O}_{7}$ as-grown, with a first annealing in oxygen, and with a second annealing in oxygen.

\subsubsection{DC magnetic susceptibility}

DC magnetization data, $M$, were collected using the superconducting quantum interference device (SQUID). $M$ was measured as a function of the temperature in a small magnetic field of $H=100$ Oe. Then, the dc magnetic susceptibility $\chi$ was determined from its definition $\chi=M / H$. Figure 4.4 shows the dc magnetic susceptibility data $\chi$ for the as-grown and annealed in oxygen $\mathrm{Gd}_{2} \mathrm{Ti}_{2} \mathrm{O}_{7}$ samples in the temperature range of 2 to $300 \mathrm{~K}$.

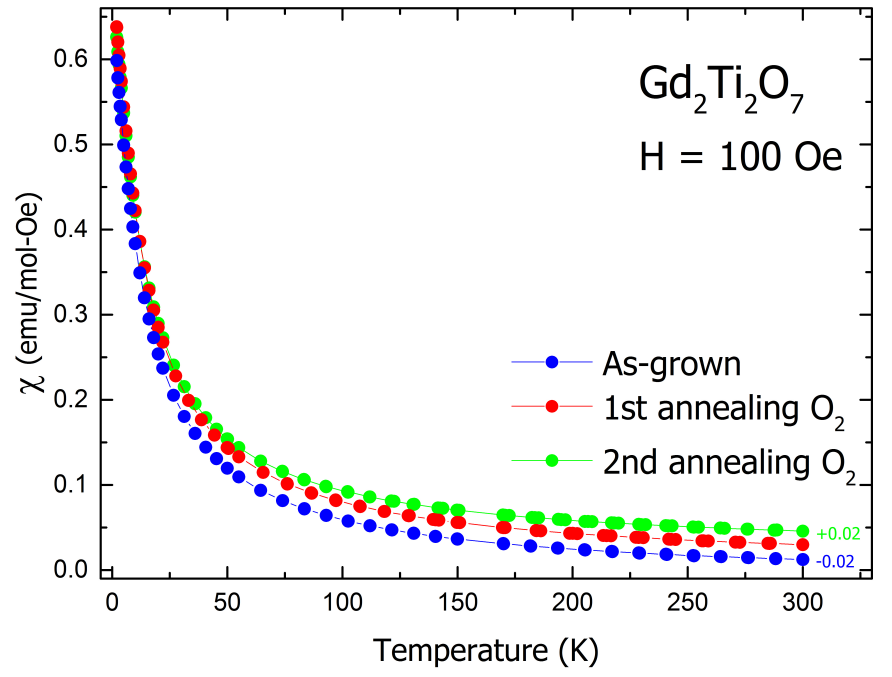

Figure 4.4: dc magnetic susceptibility $\chi$ for the as-grown, first annealing and second annealing $\mathrm{Gd}_{2} \mathrm{Ti}_{2} \mathrm{O}_{7}$ samples measured with a field $H=100$ e. The $\chi$ curves are slightly offset for clarity. 
We are interested in studying the Curie-Weiss behaviour of our material by analysing the inverse dc magnetic susceptibility $\chi^{-1}$. The linear fit of $\chi^{-1}$ provides us the values of the effective magnetic moment, $\mathrm{p}_{\text {eff }}$, and the Curie-Weiss temperature, $\theta_{\mathrm{CW}}$, which allow us to study the magnetism on the Gd sites and the degree of frustration of our different samples. Figure 4.5 shows the inverse magnetic susceptibility data, $\chi^{-1}$, and the linear fit as a function of the temperature for the as-grown and annealed in oxygen $\mathrm{Gd}_{2} \mathrm{Ti}_{2} \mathrm{O}_{7}$ samples. From the linear fit in the interval $10-300 \mathrm{~K}$, we extracted the effective moments and Curie-Weiss temperatures shown in table 4.3. For $\mathrm{Gd}_{2} \mathrm{Ti}_{2} \mathrm{O}_{7}$ with a second annealing in oxygen these results evidence that the $\mathrm{p}_{\text {eff }}$ and $\theta_{\mathrm{CW}}$ are in best agreement with $\mathrm{p}_{e f f}=$ $7.94 \mu_{B} / \mathrm{Gd}$ ion and $\theta_{\mathrm{CW}}=-9.6 \mathrm{~K}[15,39]$. The $\mathrm{p}_{\text {eff }}$ for the as-grown and the first annealing samples are 8.1 and $8.4 \mu_{B} / \mathrm{Gd}$ ion respectively which are slightly higher than $7.94 \mu_{B} / \mathrm{Gd}$. The $\theta_{\mathrm{CW}}$ for these samples also present a higher value than the literature as shown in table 4.3. By fitting the data in different temperature ranges between 10 and $300 \mathrm{~K}$ does not produce a significant change in the values of the $\mathrm{p}_{\text {eff }}$ or $\theta_{\mathrm{CW}}$. The negative values of the $\theta_{\mathrm{CW}}$ indicate the antiferromagnetic exchange interaction of the $\mathrm{Gd}^{3+}$ spins and the fact that the $\theta_{\mathrm{CW}}$ for the as-grown and the first annealing samples are "more negative" suggest a nature slightly more antiferromagnetic of the samples. Then, the frustration index $f \equiv \theta_{\mathrm{CW}} / \mathrm{T}_{\mathrm{N}}^{1}$ is determined.

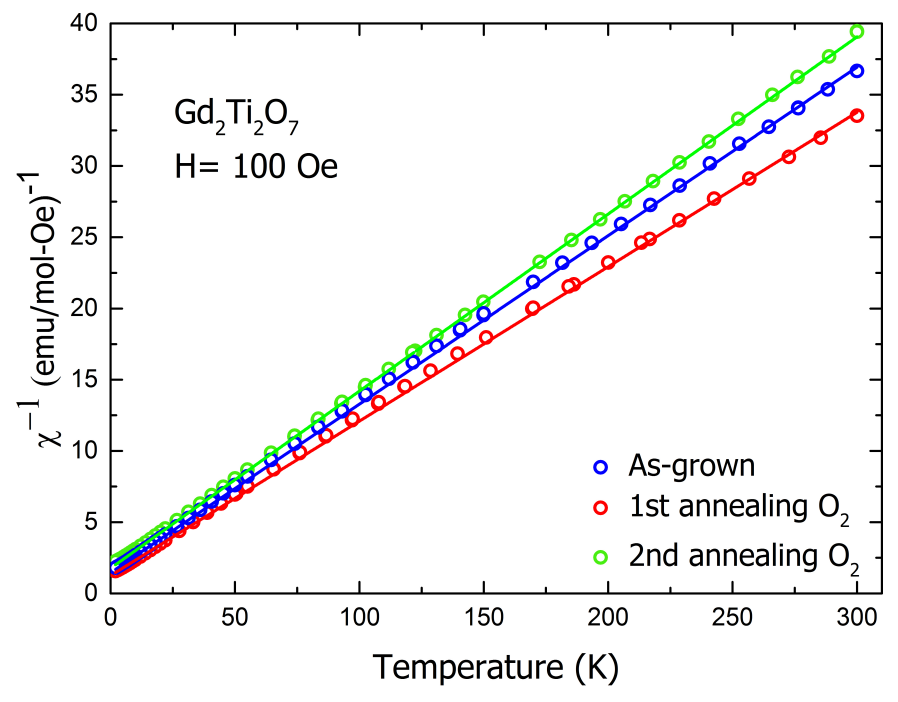

Figure 4.5: Inverse magnetic susceptibility $\chi^{-1}$ as a function of the temperature and its respective linear fit of Curie-Weiss for the as-grown (blue), first annealing (red) and second annealing in oxygen (green) $\mathrm{Gd}_{2} \mathrm{Ti}_{2} \mathrm{O}_{7}$. 
CHAPTER 4. Vacancy Defects in Oxygen-deficient $\mathrm{Gd}_{2} \mathrm{Ti}_{2} \mathrm{O}_{7}$

\begin{tabular}{c|c|c|c|c}
\hline $\mathrm{Gd}_{2} \mathrm{Ti}_{2} \mathrm{O}_{7}$ & As-grown & First annealing & Second annealing & Literature \\
\hline \hline $\mathrm{p}_{\text {eff }}\left(\mu_{B} / \mathrm{Gd}\right)$ & 8.1 & 8.4 & 7.9 & $7.94[15]$ \\
\hline$\theta_{\mathrm{CW}}(\mathrm{K})$ & -10.9 & -12.0 & -9.7 & $-9.6[15]$ \\
\hline $\mathrm{T}_{\mathrm{N}}^{1}(\mathrm{~K})$ & 1.01 & 1.18 & 1.17 & $1.05[39]$ \\
\hline Frustration index $f$ & 10.8 & 10.2 & 8.3 & $10[15]$ \\
\hline \hline
\end{tabular}

Table 4.3: Values of the Curie-Weiss temperatures $\theta_{\mathrm{CW}}$, the effective magnetic moments $\mathrm{p}_{\text {eff }}$, the transition temperatures $\mathrm{T}_{\mathrm{N}}^{1}$, and the frustration indexes $f$ for the oxygen-deficient samples of $\mathrm{Gd}_{2} \mathrm{Ti}_{2} \mathrm{O}_{7}$.

Analysing the inverse magnetic susceptibility $\chi^{-1}$ data below $22.5 \mathrm{~K}$ as shown in figure 4.6 , we notice that it starts to deviate from the linear fit approximately at $10 \mathrm{~K}$. This deviation is exhibited in all the $\mathrm{Gd}_{2} \mathrm{Ti}_{2} \mathrm{O}_{7}$ samples and it is more prominent in the sample with a second annealing in oxygen (green). This deviating at the order of $\theta_{\mathrm{CW}} \sim 10 \mathrm{~K}$ where it is expect to occur an antiferromagnetic transition of long-range order is consistent with Raju et al. [18].

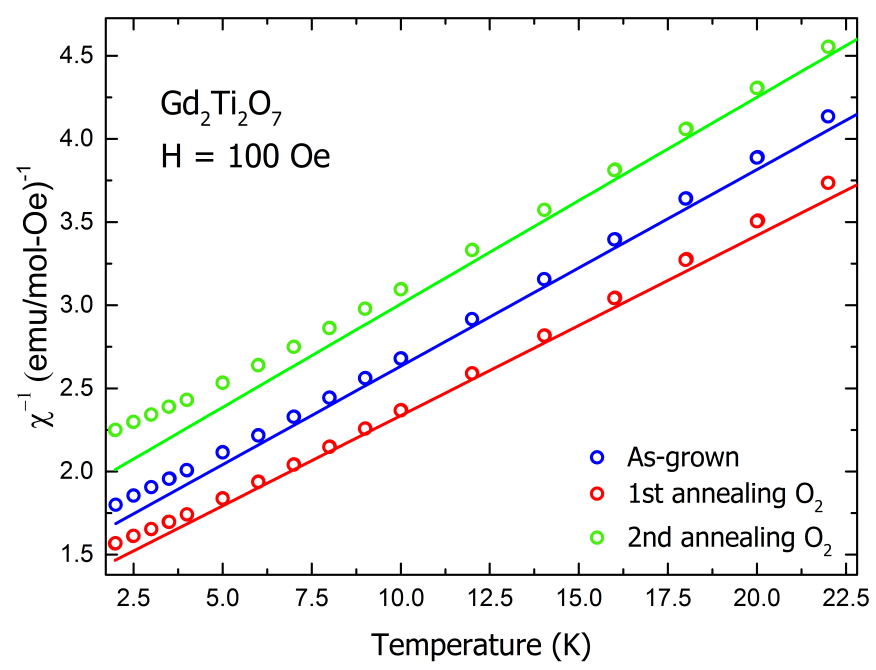

Figure 4.6: Inverse magnetic susceptibility $\chi^{-1}$ in the temperature range of $2.5-22.5 \mathrm{~K}$ for the asgrown, first annealing and second annealing in oxygen $\mathrm{Gd}_{2} \mathrm{Ti}_{2} \mathrm{O}_{7}$ samples. The $\chi^{-1}$ curves and the linear fits are shown slightly offset for clarity. 


\subsubsection{Saturation magnetization}

Magnetization measurements were conducted on the as-grown, first annealing and second annealing in oxygen $\mathrm{Gd}_{2} \mathrm{Ti}_{2} \mathrm{O}_{7}$ samples using a vibrating sample magnetometer (VSM) and a SQUID. From the magnetization data, we will discuss if the annealing in oxygen of our samples is correlated to the increase or decrease in the saturation moment. We have the assumption that a change in the saturated moment is an indicator for the presence of vacancy defects in our samples as stated in the work of Sala et al. [63]. The isothermal magnetization curves were measured above the first phase transition temperature; $\sim 1 \mathrm{~K}$; of $\mathrm{Gd}_{2} \mathrm{Ti}_{2} \mathrm{O}_{7}$.

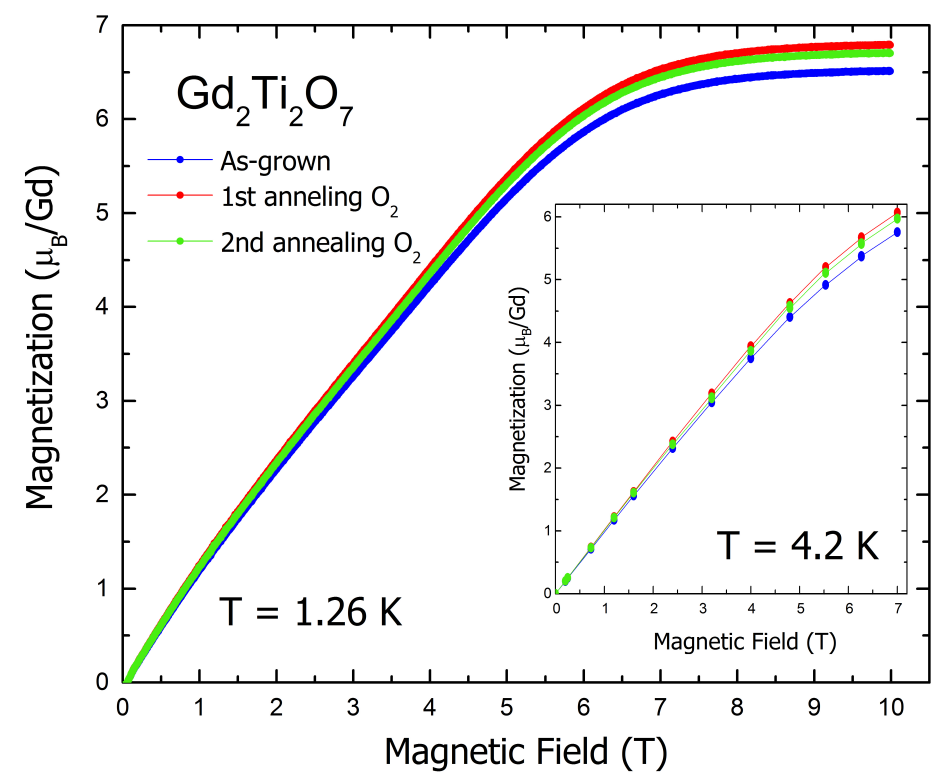

Figure 4.7: Saturation magnetization as a function of the magnetic field for the as-grown $\mathrm{Gd}_{2} \mathrm{Ti}_{2} \mathrm{O}_{7}$ (blue) and for $\mathrm{Gd}_{2} \mathrm{Ti}_{2} \mathrm{O}_{7}$ with a first (red) and second (green) annealing in oxygen at $\mathrm{T}=1.26 \mathrm{~K}$. Inset, magnetization as a function of the magnetic field for the $\mathrm{Gd}_{2} \mathrm{Ti}_{2} \mathrm{O}_{7}$ samples at $\mathrm{T}=4.2 \mathrm{~K}$.

Figure 4.7 shows the magnetization curves as a function of the applied field for the oxygen-deficient $\mathrm{Gd}_{2} \mathrm{Ti}_{2} \mathrm{O}_{7}$ samples. The saturation moments of our samples are close to the theoretical value of 7 $\mu_{B}$ expected for a state with $J=7 / 2$. At $1.26 \mathrm{~K}$, we found that for a magnetic field of $10 \mathrm{~T}$, the magnetic moment of the as-grown sample (blue) reaches a saturation value of $6.5 \mu_{B} / \mathrm{Gd}$ ion which is the lowest value among the three $\mathrm{Gd}_{2} \mathrm{Ti}_{2} \mathrm{O}_{7}$ samples. Also, we found that the annealing in oxygen leads to samples with a saturation moment of $6.8 \mu_{B} / \mathrm{Gd}$ ion (first annealing in $\mathrm{O}_{2}$ ) and $6.7 \mu_{B} / \mathrm{Gd}$ ion 
CHAPTER 4. Vacancy Defects in Oxygen-deficient $\mathrm{Gd}_{2} \mathrm{Ti}_{2} \mathrm{O}_{7}$

(second annealing in $\mathrm{O}_{2}$ ) closer to $7 \mu_{B}$. This small difference in the saturation moments is an indicator for the presence of vacancy defects in our samples [63]. Between the two annealed $\mathrm{Gd}_{2} \mathrm{Ti}_{2} \mathrm{O}_{7}$ samples, we would expect that the sample with a second annealing in oxygen produces a higher saturation moment. But as the variation between these two saturated moments is minimal, we think that this is due to demagnetization effects of the sample shapes. These first results are consistent with Petrenko et al. [49] who reported a magnetic moment of $6.8 \mu_{B} / \mathrm{Gd}$ ion at $7 \mathrm{~T}$. In the inset of figure 4.7 we show the magnetization data collected using the SQUID. At $4.2 \mathrm{~K}$, we found that the magnetization curves of our $\mathrm{Gd}_{2} \mathrm{Ti}_{2} \mathrm{O}_{7}$ samples present a much slower approach to the saturated moment of $7 \mu_{B}$. At this temperature the magnetization data continues increasing at a high rate with and reach a value of 6 $\mu_{B} / \mathrm{Gd}$ ion at a field of $7 \mathrm{~T}$. Also, by measuring magnetization for increasing and decreasing magnetic field, we found that the magnetization process is hysteresis-free for our as-grown and annealed in oxygen $\mathrm{Gd}_{2} \mathrm{Ti}_{2} \mathrm{O}_{7}$ samples. The samples employed were needle-shaped pellets in order to reduce the demagnetization correction when we measure magnetization. 


\subsection{Results of $\mathrm{Gd}_{2} \mathrm{Ti}_{2} \mathrm{O}_{7}$ grown in different atmospheres}

In this section we will continue with an enhanced study of $\operatorname{Gd}_{2} \mathrm{Ti}_{2} \mathrm{O}_{7}$ and how the oxygen vacancies affect its magnetic and thermal properties. To study oxygen-depleted pyrochlores of $\mathrm{Gd}_{2} \mathrm{Ti}_{2} \mathrm{O}_{7}$ we prepared polycrystalline $\mathrm{Gd}_{2} \mathrm{Ti}_{2} \mathrm{O}_{7}$ with the sol-gel method detailed in Chapter 3 but now the samples were sintered in three different atmospheres: oxygen, hydrogen, and air (without a particular gaseous atmosphere) that is the last heat treatment was for 24 hours at $1100^{\circ} \mathrm{C}$ in the mentioned atmospheres. Results obtained by X-ray diffraction, magnetic, and thermal measurements are reported here.

\subsubsection{Structural analysis}

In order to discuss about the vacancy defects in $\mathrm{Gd}_{2} \mathrm{Ti}_{2} \mathrm{O}_{7}$ grown in air, hydrogen $\left(\mathrm{H}_{2}\right)$, and oxygen $\left(\mathrm{O}_{2}\right)$, we analysed the $\mathrm{X}$-ray powder diffractograms of our samples. We refined the data that model the diffraction patterns to obtain the lattice parameters, the occupancies on each site of the pyrochlore lattice, and the concentration of oxygen vacancies. The X-ray diffraction patterns for $\mathrm{Gd}_{2} \mathrm{Ti}_{2} \mathrm{O}_{7}$ grown in air, $\mathrm{H}_{2}$, and $\mathrm{O}_{2}$ are shown in figure 4.8. These diffraction patterns do not present any anomalous peak excluding thus the presence of another structure different of pyrochlore.

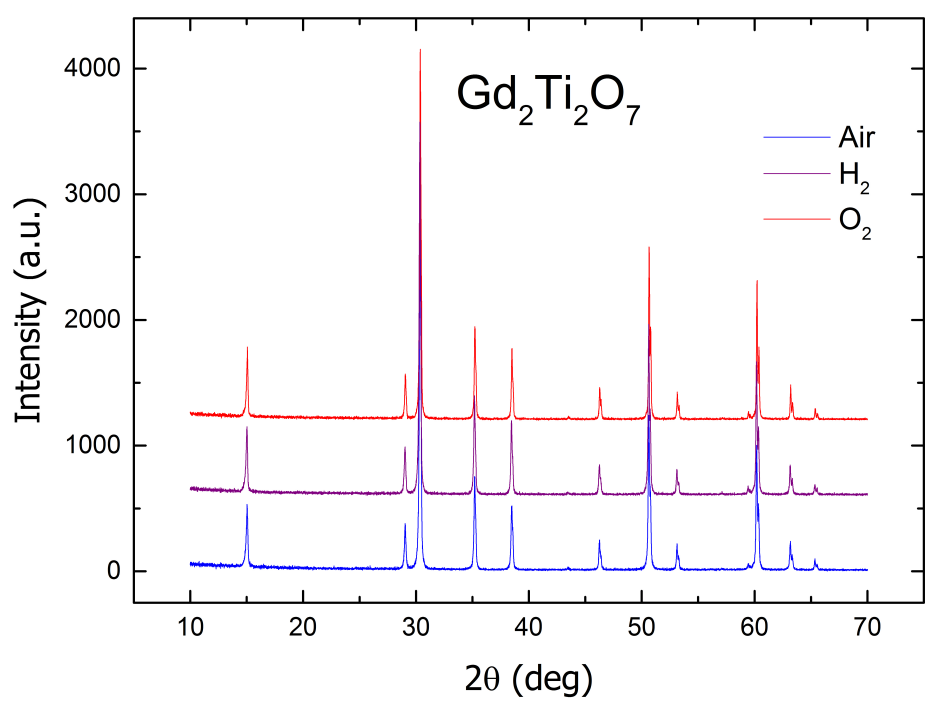

Figure 4.8: Powder $\mathrm{X}$-ray diffraction patterns for $\mathrm{Gd}_{2} \mathrm{Ti}_{2} \mathrm{O}_{7}$ grown in air, $\mathrm{H}_{2}$, and $\mathrm{O}_{2}$.

Rietveld structural refinements were carried out using the software FullProf and the graphical interface WinPLOTR. Table 4.4 shows the refinement of the structure for $\mathrm{Gd}_{2} \mathrm{Ti}_{2} \mathrm{O}_{7}$ grown in air, hy- 
CHAPTER 4. Vacancy Defects in Oxygen-deficient $\mathrm{Gd}_{2} \mathrm{Ti}_{2} \mathrm{O}_{7}$

drogen, and oxygen. While growing $\mathrm{Gd}_{2} \mathrm{Ti}_{2} \mathrm{O}_{7}$ in air leads to white powder, the growing of $\mathrm{Gd}_{2} \mathrm{Ti}_{2} \mathrm{O}_{7}$ in hydrogen and oxygen leads to black and gray samples respectively. All the diffraction data refined in the cubic pyrochlore structure with space group $F d \overline{3} m$ as shown in table 4.4. For the three $\mathrm{Gd}_{2} \mathrm{Ti}_{2} \mathrm{O}_{7}$ samples the occupancies of the Gd and Ti sites are very close to one (a site without vacancies is ideally one) which means that there are no vacancies of Gd or Ti. Also, the refinement reveals equal concentrations on the Gd and Ti sites, ruling out the stuffing of the Ti sites by Gd. Then, the refinement reveals that the oxygen vacancies were found mostly to be on the $\mathrm{O}(1)$ sites but with $\mathrm{Gd}_{2} \mathrm{Ti}_{2} \mathrm{O}_{7}$ grown in hydrogen exhibiting a higher concentration of oxygen vacancies. The occupancies of the $\mathrm{O}(2)$ sites are very close to one excluding thus any chance of vacancies in these sites. These results are consistent with Sala et al. [63] obtained for the spin ice $\mathrm{Dy}_{2} \mathrm{Ti}_{2} \mathrm{O}_{7}$. Thus, our $\mathrm{Gd}_{2} \mathrm{Ti}_{2} \mathrm{O}_{7}$ samples were found to be of an oxygen-deficient stoichiometry: $\mathrm{Gd}_{2} \mathrm{Ti}_{2} \mathrm{O}_{7-\delta}$. In addition, the chi-squared values $\chi^{2}$ of our three samples are very close to one showing that the structure refines well within the stoichiometric model. From table 4.4, the length of the lattice parameters for the $\mathrm{Gd}_{2} \mathrm{Ti}_{2} \mathrm{O}_{7}$ samples are consistent with $[39,40]$ and present a negligible difference among them. It is difficult to define a behaviour of the lattice parameter as a function of the oxygen concentration; however, we found that the length of the lattice parameter increases for $\mathrm{Gd}_{2} \mathrm{Ti}_{2} \mathrm{O}_{7}$ grown in oxygen compared to $\mathrm{Gd}_{2} \mathrm{Ti}_{2} \mathrm{O}_{7}$ grown in air.

\begin{tabular}{c|c|c|c}
\hline $\mathrm{Gd}_{2} \mathrm{Ti}_{2} \mathrm{O}_{7}$ grown in & $\mathrm{H}_{2}$ & Air & $\mathrm{O}_{2}$ \\
\hline \hline Colour & Black & White & Gray \\
\hline Space group & $F d \overline{3} m$ & $F d \overline{3} m$ & $F d \overline{3} m$ \\
\hline Lattice parameter $(\AA)$ & 10.181 & 10.184 & 10.181 \\
\hline $\mathrm{Gd}$ & 0.99 & 0.99 & 0.99 \\
\hline $\mathrm{Ti}$ & 1.01 & 1.05 & 1.04 \\
\hline $\mathrm{O}(1)$ & 0.73 & 0.81 & 0.81 \\
\hline $\mathrm{O}(2)$ & 0.96 & 0.95 & 0.99 \\
\hline$\chi^{2}$ & 2.18 & 1.57 & 1.60 \\
\hline \hline
\end{tabular}

Table 4.4: Refinement of the structure of $\mathrm{Gd}_{2} \mathrm{Ti}_{2} \mathrm{O}_{7}$ grown in air, $\mathrm{H}_{2}$, and $\mathrm{O}_{2}$ showing the space groups, the lattice parameters, the occupancies of each site, and the values of chi-squared. 


\subsubsection{AC magnetic susceptibility}

For the $\mathrm{Gd}_{2} \mathrm{Ti}_{2} \mathrm{O}_{7}$ samples grown in air, $\mathrm{H}_{2}$, and $\mathrm{O}_{2}$, we report in figure 4.9 the ac magnetic susceptibility $\chi_{a c}$ as a function of the temperature. The collected data of the $\mathrm{Gd}_{2} \mathrm{Ti}_{2} \mathrm{O}_{7}$ samples display a peak like anomaly close to $1 \mathrm{~K}$ in agreement with $[15,39] . \mathrm{Gd}_{2} \mathrm{Ti}_{2} \mathrm{O}_{7}$ samples grown in different atmospheres present a broader peak in contrast to the sharp magnetic transition of $\mathrm{Gd}_{2} \mathrm{Ti}_{2} \mathrm{O}_{7}$ annealed in oxygen of the previous section (figure 4.2, green). The transition at $1 \mathrm{~K}$ is identified as a partial ordering of the Gd spins in the Kagomé planes of the pyrochlore lattice [61]. For $\operatorname{Gd}_{2} \operatorname{Ti}_{2} \mathrm{O}_{7}$ grown in air (blue) and $\mathrm{O}_{2}$ (red) the transitions occur at $\mathrm{T}_{\mathrm{N}}^{1}=0.99$ and $1.02 \mathrm{~K}$ respectively. The oxygen-depleted $\mathrm{Gd}_{2} \mathrm{Ti}_{2} \mathrm{O}_{7}$ grown in $\mathrm{H}_{2}$ (purple) shows an unexpected defined peak of phase transition at $\mathrm{T}_{\mathrm{N}}^{1}=0.98 \mathrm{~K}$. Even though the difference among the reported transition temperatures $\mathrm{T}_{\mathrm{N}}^{1}$ is negligible, there is a remarkable difference on the peak intensity of the $\chi_{a c}$ curve of $\mathrm{Gd}_{2} \mathrm{Ti}_{2} \mathrm{O}_{7}$ grown in $\mathrm{H}_{2}$ compared to the $\mathrm{Gd}_{2} \mathrm{Ti}_{2} \mathrm{O}_{7}$ grown in air and $\mathrm{O}_{2}$ which present an analogue behaviour after the phase transition. The $\chi_{a c}$ collected data of $\mathrm{Gd}_{2} \mathrm{Ti}_{2} \mathrm{O}_{7}$ grown in air and $\mathrm{O}_{2}$ converge to the same values after their ordering temperatures.

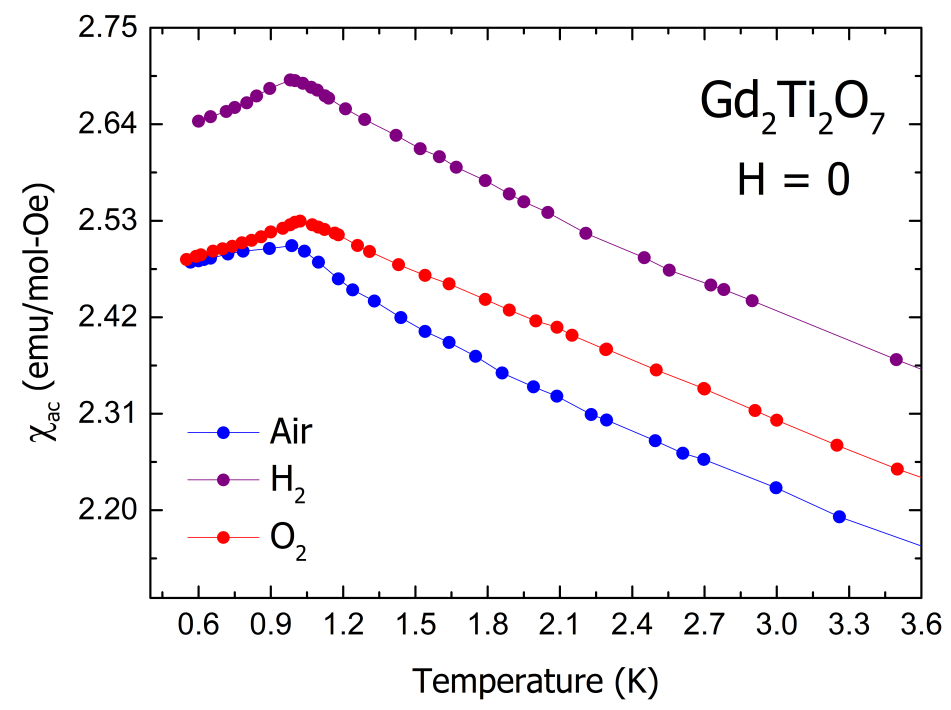

Figure 4.9: Temperature dependence of the ac magnetic susceptibility $\chi_{a c}$ for $\mathrm{Gd}_{2} \mathrm{Ti}_{2} \mathrm{O}_{7}$ grown in different atmospheres: air (blue), $\mathrm{H}_{2}$ (purple), and $\mathrm{O}_{2}$ (red) measured at zero field.

The left side of figure 4.10 shows the complete ac susceptibility data of figure 4.9. Here, the $\chi_{a c}$ curves of $\mathrm{Gd}_{2} \mathrm{Ti}_{2} \mathrm{O}_{7}$ grown in air, $\mathrm{H}_{2}$, and $\mathrm{O}_{2}$ present an analogue behaviour to the curves presented in 
figure 4.3. The right side of figure 4.10 shows the $\chi_{a c}$ data without the offset. Here, we notice better that the $\chi_{a c}$ curves of $\mathrm{Gd}_{2} \mathrm{Ti}_{2} \mathrm{O}_{7}$ grown in air (blue) and $\mathrm{O}_{2}$ (red) are superimposed and converge to the same values of susceptibility. As in the previous section, the second magnetic transition at $\mathrm{T}_{\mathrm{N}}^{2} \sim$ $0.75 \mathrm{~K}$ was not found for any of our $\mathrm{Gd}_{2} \mathrm{Ti}_{2} \mathrm{O}_{7}$ samples.
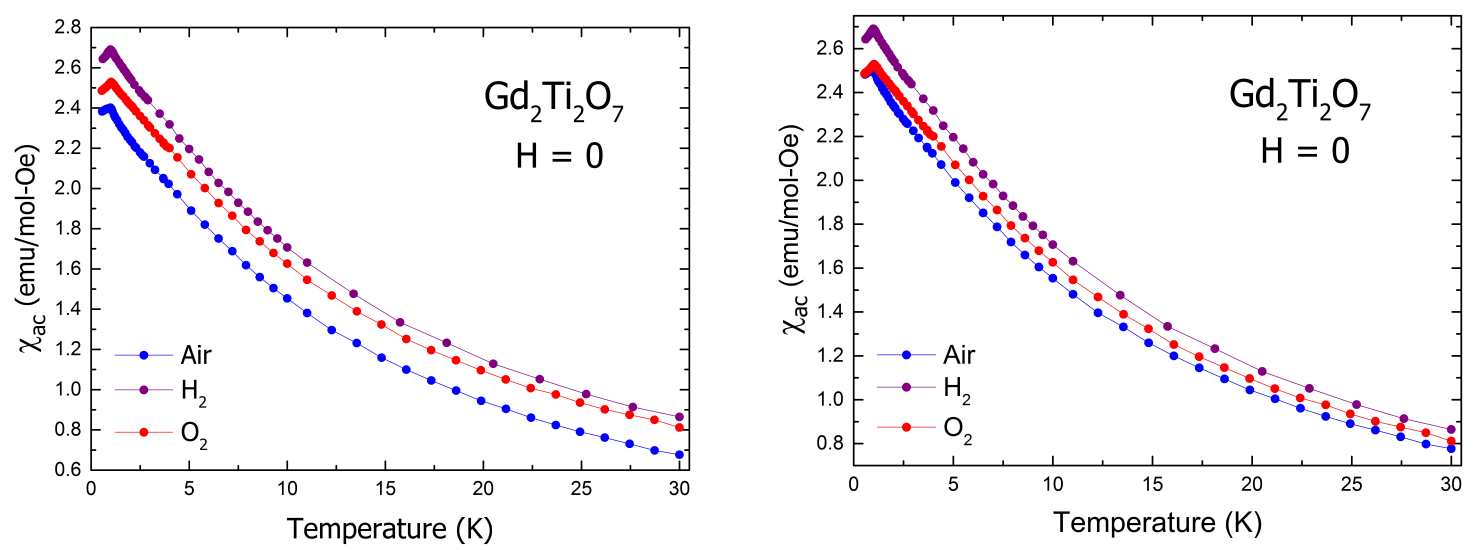

Figure 4.10: Left: Full data of ac magnetic susceptibility $\chi_{a c}$ as a function of the temperature for $\mathrm{Gd}_{2} \mathrm{Ti}_{2} \mathrm{O}_{7}$ grown in air, $\mathrm{H}_{2}$, and $\mathrm{O}_{2}$ measured at zero field. $\chi_{a c}$ curves present a slight offset for clarity. Right: Raw $\chi_{a c}$ data of the $\mathrm{Gd}_{2} \mathrm{Ti}_{2} \mathrm{O}_{7}$ samples.

The values of the transition temperatures for the $\mathrm{Gd}_{2} \mathrm{Ti}_{2} \mathrm{O}_{7}$ samples are presented in table 4.5. These ordering temperatures $\mathrm{T}_{\mathrm{N}}^{1}$ present a slight variation of $\pm 0.02 \mathrm{~K}$ among them.

\begin{tabular}{c|c|c|c}
\hline $\mathrm{Gd}_{2} \mathrm{Ti}_{2} \mathrm{O}_{7}$ grown in & $\mathrm{H}_{2}$ & Air & $\mathrm{O}_{2}$ \\
\hline \hline $\mathrm{T}_{\mathrm{N}}^{1}(\mathrm{~K})$ & 0.98 & 0.99 & 1.02 \\
\hline \hline
\end{tabular}

Table 4.5: Transition temperatures $\mathrm{T}_{\mathrm{N}}^{1}$ for $\mathrm{Gd}_{2} \mathrm{Ti}_{2} \mathrm{O}_{7}$ samples grown in $\mathrm{H}_{2}$, air, and $\mathrm{O}_{2}$. 
The field dependence of the ac magnetic susceptibility $\chi_{a c}$ measured at different temperatures is shown in figure 4.11. Since previous $\chi_{a c}$ results of Ramirez et al. [15] were reported for stoichiometric $\mathrm{Gd}_{2} \mathrm{Ti}_{2} \mathrm{O}_{7}$, we performed experiments on the $\mathrm{Gd}_{2} \mathrm{Ti}_{2} \mathrm{O}_{7}$ grown in oxygen which has less vacancies and a better stoichiometry according to table 4.4. From figure 4.11, we found that as the field reaches a value around $6 \mathrm{~T}$, all the susceptibility curves exhibit a sharp downturn and then go to zero. At 1.11 (black) and $0.90 \mathrm{~K}$ (red) both $\chi_{a c}$ curves resemble each other and do not present a visible peak between 0 and $3 \mathrm{~T}$ but at $0.90 \mathrm{~K}$ there is a small hump for a field close to $2.3 \mathrm{~T}$. At these temperatures it was not collected the $\chi_{a c}$ data between 3 and $6 \mathrm{~T}$ since there is no any visible hump according to [15]. The $\chi_{a c}$ curves at 0.67 (blue) and $0.50 \mathrm{~K}$ (green) exhibit a peak centered at fields of 2.9 and 3 $\mathrm{T}$ respectively. The $\chi_{a c}$ data at $0.50 \mathrm{~K}$ presents a higher and sharper peak compared with the data at $0.67 \mathrm{~K}$. Thus, as the temperature is decreased the susceptibility peak gets sharper and moves with the increase of the magnetic field. The positions of the peaks at a fixed field are consistent with the field-induced transitions of the phase diagram for $\mathrm{Gd}_{2} \mathrm{Ti}_{2} \mathrm{O}_{7}$ reported by Ramirez et al. [15].

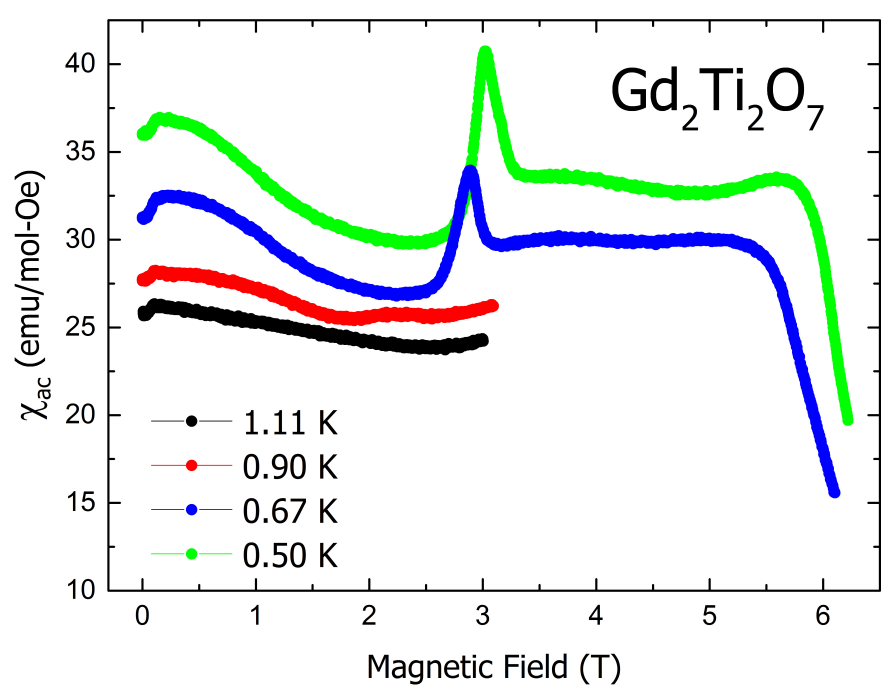

Figure 4.11: Field dependence of the ac susceptibility $\chi_{a c}(\mathrm{H})$ for $\mathrm{Gd}_{2} \mathrm{Ti}_{2} \mathrm{O}_{7}$ grown in oxygen atmosphere at temperatures above and below the transition temperature $\mathrm{T}_{\mathrm{N}}^{1}=1.02 \mathrm{~K}$. The $0.50,0.90$, and $1.11 \mathrm{~K}$ data are offset by $+3.5,-3.0$, and $-5.0 \mathrm{emu} / \mathrm{mol}-\mathrm{Oe}$, respectively, for clarity. 


\subsubsection{DC magnetic susceptibility}

As in the previous section, we obtained the Curie-Weiss parameters by means of the linear fit of the inverse dc susceptibility $\chi^{-1}$. In figure 4.12 it is presented the temperature dependence of $\chi^{-1}$ for $\mathrm{Gd}_{2} \mathrm{Ti}_{2} \mathrm{O}_{7}$ grown in air, $\mathrm{H}_{2}$ and $\mathrm{O}_{2}$ measured in a magnetic field of 100 Oe. The $\chi^{-1}$ data present a tendency to increase for $\mathrm{Gd}_{2} \mathrm{Ti}_{2} \mathrm{O}_{7}$ grown in $\mathrm{O}_{2}$ (red) that is a higher slope of the linear fit in comparison with the samples grown in air (blue) and $\mathrm{H}_{2}$ (purple).

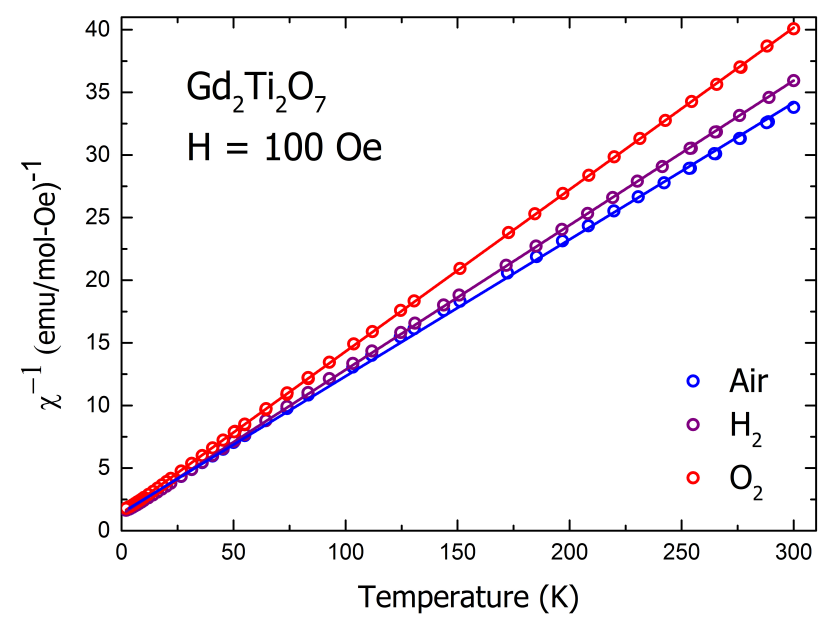

Figure 4.12: Inverse susceptibility $\chi^{-1}$ as a function of the temperature and its respective linear fit of Curie-Weiss for $\mathrm{Gd}_{2} \mathrm{Ti}_{2} \mathrm{O}_{7}$ grown in air (blue), $\mathrm{H}_{2}$ (purple) and $\mathrm{O}_{2}$ (red) measured in an applied field of 100 Oe.

From the linear fit in the interval $10-300 \mathrm{~K}$, we obtained the effective moments $\mathrm{p}_{\text {eff }}$ and the Curie-Weiss temperatures $\theta_{\mathrm{CW}}$. Also, in order to measure the degree of frustration of our $\mathrm{Gd}_{2} \mathrm{Ti}_{2} \mathrm{O}_{7}$ samples we used the transition temperatures $\mathrm{T}_{\mathrm{N}}^{1}$ to obtain the frustration index $f$. These values are shown in table 4.6. The effective magnetic moment for $\mathrm{Gd}_{2} \mathrm{Ti}_{2} \mathrm{O}_{7}$ grown in $\mathrm{O}_{2}$ is $7.9 \mu_{B} / \mathrm{Gd}$ ion very close to the theoretical value of $7.94 \mu_{B} / \mathrm{Gd}$ ion for $\mathrm{J}=7 / 2$ [9]. On the other hand, for $\mathrm{Gd}_{2} \mathrm{Ti}_{2} \mathrm{O}_{7}$ grown in $\mathrm{H}_{2}$ and air the values of $\mathrm{p}_{e f f}$ exceed unexpectedly the theoretical value of $7.94 \mu_{B} / \mathrm{Gd}$ ion. The frustration indexes $f$ in table 4.6 show that $\mathrm{Gd}_{2} \mathrm{Ti}_{2} \mathrm{O}_{7}$ grown in air with $f=13.9$ presents a higher degree of geometric frustration compared with the samples grown in $\mathrm{O}_{2}$ and $\mathrm{H}_{2}$. 
CHAPTER 4. Vacancy Defects in Oxygen-deficient $\mathrm{Gd}_{2} \mathrm{Ti}_{2} \mathrm{O}_{7}$

\begin{tabular}{c|c|c|c|c}
\hline $\mathrm{Gd}_{2} \mathrm{Ti}_{2} \mathrm{O}_{7}$ grown in & $\mathrm{H}_{2}$ & Air & $\mathrm{O}_{2}$ & Literature \\
\hline \hline $\mathrm{p}_{\text {eff }}\left(\mu_{B} / \mathrm{Gd}\right)$ & 8.3 & 8.5 & 7.9 & $7.94[15]$ \\
\hline$\theta_{\mathrm{CW}}(\mathrm{K})$ & -11.5 & -13.8 & -10.9 & $-9.6[15]$ \\
\hline $\mathrm{T}_{\mathrm{N}}^{1}(\mathrm{~K})$ & 0.98 & 0.99 & 1.02 & $1.05[39]$ \\
\hline Frustration index $f$ & 11.7 & 13.9 & 10.7 & $10[15]$ \\
\hline \hline
\end{tabular}

Table 4.6: Values of the Curie-Weiss temperatures $\theta_{\mathrm{CW}}$, the effective magnetic moments $\mathrm{p}_{\text {eff }}$, the transition temperatures $\mathrm{T}_{\mathrm{N}}^{1}$, and the frustration indexes $f$ for $\mathrm{Gd}_{2} \mathrm{Ti}_{2} \mathrm{O}_{7}$ grown in $\mathrm{H}_{2}$, air, and $\mathrm{O}_{2}$.

We present in figure 4.13 the low-temperature regime of the inverse susceptibility for the $\mathrm{Gd}_{2} \mathrm{Ti}_{2} \mathrm{O}_{7}$ samples. Below $5 \mathrm{~K}$ the collected data of $\mathrm{Gd}_{2} \mathrm{Ti}_{2} \mathrm{O}_{7}$ grown in $\mathrm{H}_{2}$, air, and $\mathrm{O}_{2}$ starts to deviate from the linear behaviour and for the samples grown in $\mathrm{O}_{2}$ and $\mathrm{H}_{2}$ there is a more pronounced deviation which is related with a long-range antiferromagnetic transition [18].

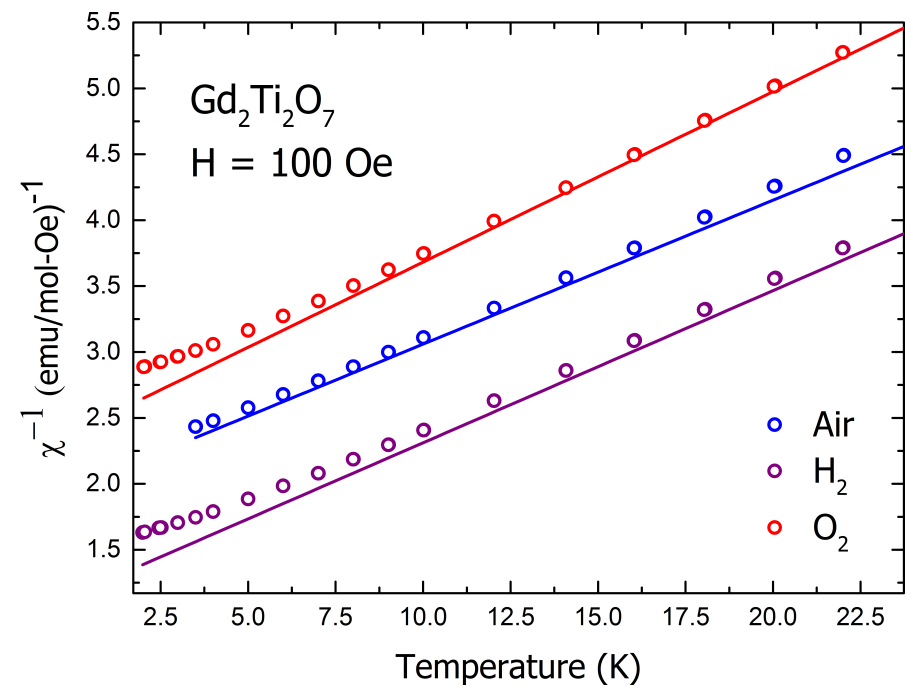

Figure 4.13: Inverse magnetic susceptibility $\chi^{-1}$ between $2 \mathrm{~K}$ and $22.5 \mathrm{~K}$ for $\mathrm{Gd}_{2} \mathrm{Ti}_{2} \mathrm{O}_{7}$ grown in $\mathrm{H}_{2}$, air, and $\mathrm{O}_{2}$ measured in an applied field of 100 Oe. 


\subsubsection{Saturation magnetization}

Magnetization data for $\mathrm{Gd}_{2} \mathrm{Ti}_{2} \mathrm{O}_{7}$ grown in air, $\mathrm{H}_{2}$, and $\mathrm{O}_{2}$ was collected using a vibrating sample magnetometer (VSM). Figure 4.14 displays the magnetization curves as a function of the applied field. For our $\mathrm{Gd}_{2} \mathrm{Ti}_{2} \mathrm{O}_{7}$ samples, we found that the magnetization process is essentially hysteresis-free and does not depend on sample history. At $4.2 \mathrm{~K}$, above the transition temperature $1 \mathrm{~K}$, the magnetization of each $\mathrm{Gd}_{2} \mathrm{Ti}_{2} \mathrm{O}_{7}$ sample shows a slow approach to the theoretical expected value of $7 \mu_{B}$ and starts to saturate for magnetic fields higher than $12 \mathrm{~T}$. Specifically, for $\mathrm{Gd}_{2} \mathrm{Ti}_{2} \mathrm{O}_{7}$ grown in air the saturation moment reaches a value of $6.7 \mu_{B} / \mathrm{Gd}$ ion in a field of $13 \mathrm{~T}$. Remarkably, for $\mathrm{Gd}_{2} \mathrm{Ti}_{2} \mathrm{O}_{7}$ grown in $\mathrm{O}_{2}$, in an applied field of $14 \mathrm{~T}$, the saturation moment reaches a value of $7 \mu_{B} / \mathrm{Gd}$ ion which is slightly higher than the former. Both saturated moments are consistent with the theoretical value of $7 \mu_{B}$ and even though the difference between them is minimal it leads us to think about the reduced magnetic moment on the $\mathrm{Gd}$ sites. However, for $\mathrm{Gd}_{2} \mathrm{Ti}_{2} \mathrm{O}_{7}$ grown in air we found an unexpected saturation moment of $7.5 \mu_{B} / \mathrm{Gd}$ ion which certainly has no physical meaning. Our data display a pattern behaviour in which the annealing in oxygen reduces the oxygen vacancies and increases the saturation moment to reach the expected theoretical value. These results are consistent with our previous results of figure 4.7 .

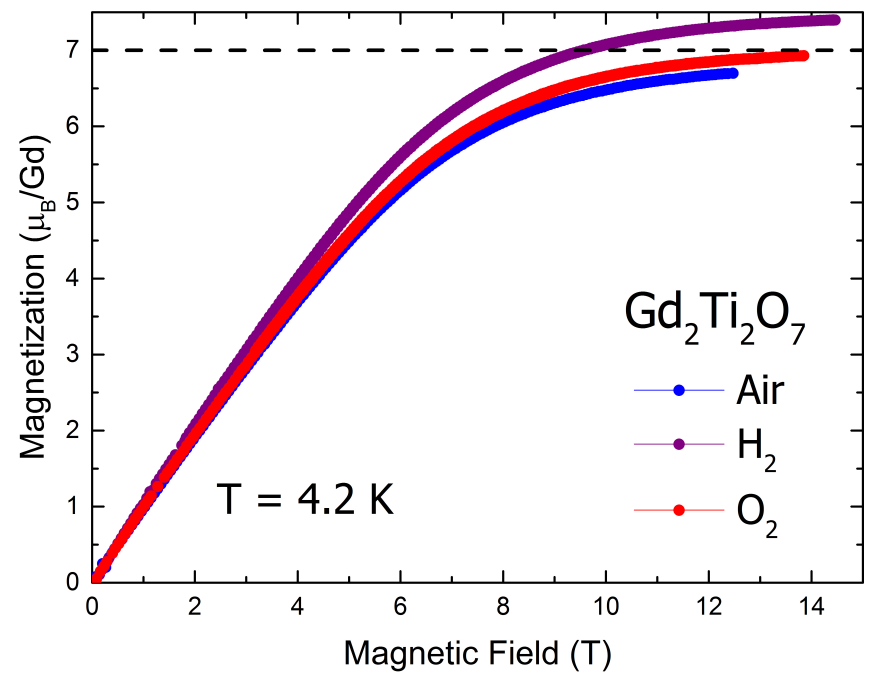

Figure 4.14: Saturation magnetization as a function of the applied field for $\mathrm{Gd}_{2} \mathrm{Ti}_{2} \mathrm{O}_{7}$ grown in air, $\mathrm{H}_{2}$, and $\mathrm{O}_{2}$ measured at $4.2 \mathrm{~K}$. Dash line indicates the expected theoretical value of $7 \mu_{B} / \mathrm{Gd}$ ion. 


\subsubsection{Specific heat}

The specific heat measurements were obtained using a Quantum Design PPMS calorimeter equipped with a ${ }^{3} \mathrm{He}-{ }^{4} \mathrm{He}$ dilution refrigerator. We report the data for the $\mathrm{Gd}_{2} \mathrm{Ti}_{2} \mathrm{O}_{7}$ sample grown in $\mathrm{O}_{2}$ atmosphere because it has less concentration of oxygen vacancies and thus a stoichiometry closer to the ideal one. Figure 4.15 shows the magnetic specific heat data divided by the temperature $C_{m}(\mathrm{~T}) / \mathrm{T}$ measured in a zero applied field. The $C_{m}$ data was obtained by subtracting the lattice specific heat $C_{\text {lat }} \propto \mathrm{T}^{3}$ from the total specific heat $C$. To compute an approximate value of $C_{\text {lat }}$ we fit $C$ with a $\mathrm{T}^{3}$ dependence between 10 and $15 \mathrm{~K}$. Then, as shown in figure 4.15 we found in the $C_{m}$ data two sharp peaks with similar amplitudes at temperatures $\mathrm{T}_{\mathrm{N}}^{1}=1.04 \mathrm{~K}$ and $\mathrm{T}_{\mathrm{N}}^{2}=0.73 \mathrm{~K}$ that correspond to two consecutive magnetic transitions as reported previously $[39,51]$. The transition observed at $\mathrm{T}_{\mathrm{N}}^{1}$ $=1.04 \mathrm{~K}$ corresponds to a partial magnetic ordering of the Gd spins [40], whereas the transition at $\mathrm{T}_{\mathrm{N}}^{2}=0.73 \mathrm{~K}$ concerns a quarter of the $\mathrm{Gd}$ spins which are disordered above $0.73 \mathrm{~K}$ and very weakly ordered below [40]. Our $C_{m} / \mathrm{T}$ data reaches a value of $11 \mathrm{~J} \cdot \mathrm{K}^{-2} \cdot \mathrm{mol}^{-1}$ at the temperature of the first transition $1.04 \mathrm{~K}$ and a value of $10 \mathrm{~J} \cdot \mathrm{K}^{-2} \cdot \mathrm{mol}^{-1}$ at the temperature of the second transition $0.73 \mathrm{~K}$ in excellent agreement with the data reported for polycrystalline stoichiometric $\mathrm{Gd}_{2} \mathrm{Ti}_{2} \mathrm{O}_{7}$ in [39].

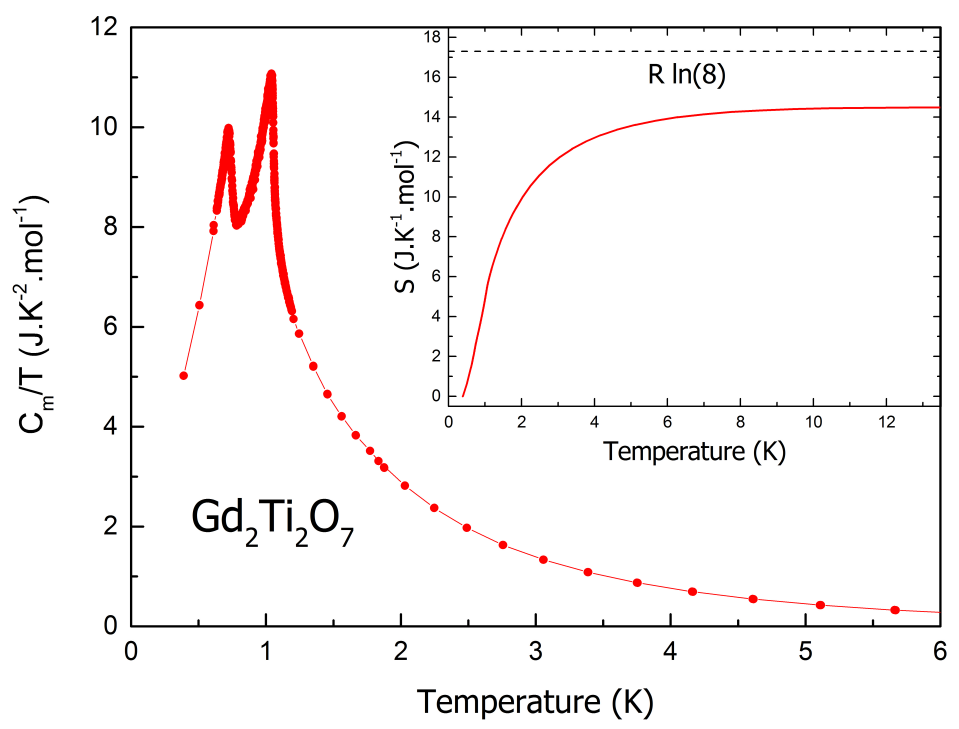

Figure 4.15: Temperature dependence of the magnetic specific heat divided by the temperature $C_{m}(\mathrm{~T}) / \mathrm{T}$ for $\mathrm{Gd}_{2} \mathrm{Ti}_{2} \mathrm{O}_{7}$ measured in zero field. Two phase transitions occur at $\mathrm{T}_{\mathrm{N}}^{1}=1.04 \mathrm{~K}$ and $\mathrm{T}_{\mathrm{N}}^{2}$ $=0.73 \mathrm{~K}$. The inset displays the entropy variation as a function of the temperature. 
The inset of figure 4.15 shows the temperature dependence of the variation of the magnetic entropy $\mathrm{S}$, obtained by integrating numerically $C_{m}(\mathrm{~T}) / \mathrm{T}$ versus temperature. Above $10 \mathrm{~K}$, the total magnetic entropy is not fully recovered and reaches a value of $14.5 \mathrm{~J} / \mathrm{mol} \mathrm{K}$ which is approximately $84 \%$ of the expected entropy $\mathrm{R} \ln (8)=17.3 \mathrm{~J} / \mathrm{mol} \mathrm{K}$. Then, it seems that the magnetic entropy has reduced its maximum value and thus there is a missing entropy which is related to the oxygen vacancies of $\mathrm{Gd}_{2} \mathrm{Ti}_{2} \mathrm{O}_{7}$. Also, approximately $15 \%$ and $30 \%$ of the expected $\mathrm{R} \ln (8)$ is recovered up to the temperatures of the peaks $\mathrm{T}_{\mathrm{N}}^{2}=0.73 \mathrm{~K}$ and $\mathrm{T}_{\mathrm{N}}^{1}=1.04 \mathrm{~K}$ respectively. Thus, short-range order interactions are expected for temperatures above of the first peak according to [39].

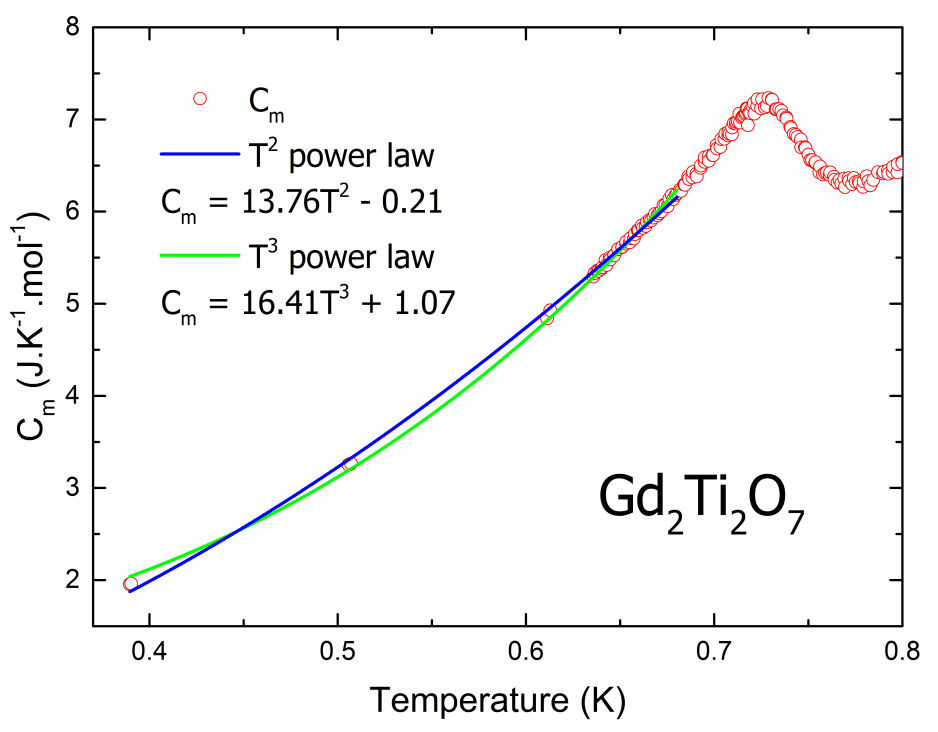

Figure 4.16: Magnetic specific heat $C_{m}$ of $\mathrm{Gd}_{2} \mathrm{Ti}_{2} \mathrm{O}_{7}$ as a function of the temperature below the second transition $\mathrm{T}_{\mathrm{N}}^{2}=0.73 \mathrm{~K}$. The $\mathrm{T}^{2}$ power law previously proposed and a $\mathrm{T}^{3}$ power law are plotted between 0.68 and $0.39 \mathrm{~K}$.

By analysing $C_{m}$ in the low-temperature regime that is below the second transition of $\operatorname{Gd}_{2} \mathrm{Ti}_{2} \mathrm{O}_{7}$, we compare the different temperature dependences observed in different antiferromagnet pyrochlores in previous works $[11,79,80]$. Figure 4.16 shows, up to $0.68 \mathrm{~K}$, the $\mathrm{T}^{2}$ and $\mathrm{T}^{3}$ power law behaviours for the specific heat $C_{m}$. Even though the $\mathrm{T}^{2}$ dependence of $C_{m}$ for $\mathrm{Gd}_{2} \mathrm{Ti}_{2} \mathrm{O}_{7}$ is expected and has been discussed by considering a density of magnetic states that is linear with energy [79], we study the $\mathrm{T}^{3}$ behaviour due to the antiferromagnetic magnons contribution since in the temperature range $0.39 \mathrm{~K} \leq \mathrm{T} \leq 0.68 \mathrm{~K}$ both power laws resemble each other. Then, the calculated $\mathrm{T}^{2}$ fit of $C_{m}$ is: $\mathrm{AT}^{2}$ 
with $\mathrm{A}=13.76 \mathrm{~J} \cdot \mathrm{K}^{-3} \cdot \mathrm{mol}^{-1}\left(\mathrm{R}^{2}=0.99\right)$. On the other side, the $\mathrm{T}^{3}$ fit results in a dependence: $\Lambda \mathrm{T}^{3}$ with $\Lambda=16.41 \mathrm{~J} \cdot \mathrm{K}^{-4} \cdot \mathrm{mol}^{-1}\left(\mathrm{R}^{2}=0.99\right)$. Then, from the $\mathrm{T}^{3}$ fit and by using equation (41) with $a$ $=10.181 \AA$ we found that the velocity of magnons in $\mathrm{Gd}_{2} \mathrm{Ti}_{2} \mathrm{O}_{7}$ is $v_{s w}=46.2 \mathrm{~m} / \mathrm{s}$.

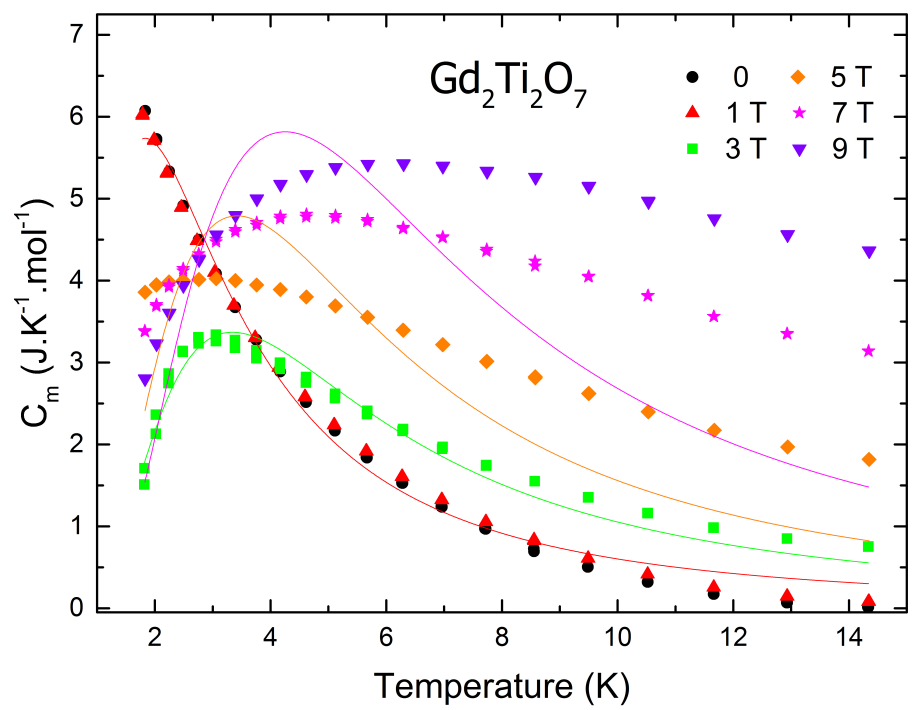

Figure 4.17: Temperature dependence of the magnetic specific heat $C_{m}$ of $\operatorname{Gd}_{2} \mathrm{Ti}_{2} \mathrm{O}_{7}$ measured with different magnetic fields. All the $C_{m}$ curves present a Schottky anomaly above the transition temperature $T_{\mathrm{N}}^{1}=1.04 \mathrm{~K}$. The solid lines represent the fits of the data to the Schottky specific heat.

We also performed specific heat measurements in magnetic field of $\mathrm{Gd}_{2} \mathrm{Ti}_{2} \mathrm{O}_{7}$ above its first transition temperature $\mathrm{T}_{\mathrm{N}}^{1}=1.04 \mathrm{~K}$. Figure 4.17 displays the magnetic specific heat $C_{m}$ as a function of the temperature measured with fields between 0 and $9 \mathrm{~T}$. Applying a magnetic field of $1 \mathrm{~T}$, the values of $C_{m}$ present no differences with the data measured at zero field. Increasing the field up to $3 \mathrm{~T}$ a broad peak emerges centered at about $3 \mathrm{~K}$. This peak becomes broader and shifts to higher temperatures with higher fields as is shown for 5, 7, and $9 \mathrm{~T}$. This high-field behaviour resembles a Schottky anomaly as reported by [81]. Then, to describe the broad peak we use the simple model of two energy levels; equation (43); for the Schottky specific heat. The calculated fits (solid lines) for the high-field $C_{m}$ data result in sharper peaks with higher maximum values of $C_{m}$ which do not fit well the data. 


\subsection{Discussion}

The results of the refinement of the X-ray patterns presented in table 4.1 and 4.4 for $\mathrm{Gd}_{2} \mathrm{Ti}_{2} \mathrm{O}_{7}$ with annealings in oxygen and for $\mathrm{Gd}_{2} \mathrm{Ti}_{2} \mathrm{O}_{7}$ grown in different atmospheres respectively reveal that the oxygen vacancies are located on the site $\mathrm{O}(1)$ of the pyrochlore lattice. We found a higher level of oxygen vacancies in the $\mathrm{Gd}_{2} \mathrm{Ti}_{2} \mathrm{O}_{7}$ sample with a second annealing in oxygen (occupancy 0.66) and also in $\mathrm{Gd}_{2} \mathrm{Ti}_{2} \mathrm{O}_{7}$ grown in hydrogen atmosphere (occupancy 0.73). The sample grown in oxygen atmosphere reaches a better stoichiometry and possesses less oxygen vacancies (occupancy 0.81). The oxygen deficiency suggests that in our $\mathrm{Gd}_{2} \mathrm{Ti}_{2} \mathrm{O}_{7}$ samples there is a slight distortion in the lattice as reported for the spin ice $\mathrm{Dy}_{2} \mathrm{Ti}_{2} \mathrm{O}_{7}$ in [63]. Then, we assume that the Gd ions relax away from the oxygen vacancy $\mathrm{O}(1)$ along $\langle 111\rangle$ directions similarly to the structure of $\mathrm{Dy}_{2} \mathrm{Ti}_{2} \mathrm{O}_{7}$ with oxygen vacancies shown in figure 2.27. In addition, the lattice parameters obtained are close to $10.184 \AA$ and present negligible differences among them.

From our ac magnetic susceptibility measurements we found that the transition temperatures $\mathrm{T}_{\mathrm{N}}^{1} \sim$ $1 \mathrm{~K}$ for all the $\mathrm{Gd}_{2} \mathrm{Ti}_{2} \mathrm{O}_{7}$ samples in the two different batch consistent with results of [15]. We found particular differences in each one of the batch. For the $\mathrm{Gd}_{2} \mathrm{Ti}_{2} \mathrm{O}_{7}$ samples with annealing in oxygen, we found that the first and second annealing in oxygen does not alter the transition temperatures; 1.18 and $1.17 \mathrm{~K}$; and also that the peaks of the transition are well defined. On the other side, the $\mathrm{Gd}_{2} \mathrm{Ti}_{2} \mathrm{O}_{7}$ samples grown in different atmospheres again present no variation on the transition temperatures but now the peaks of transition are broader. Also, the $\chi_{a c}$ curve of $\mathrm{Gd}_{2} \mathrm{Ti}_{2} \mathrm{O}_{7}$ grown in hydrogen differs largely from the ones of $\mathrm{Gd}_{2} \mathrm{Ti}_{2} \mathrm{O}_{7}$ grown in air and oxygen. For $\mathrm{Gd}_{2} \mathrm{Ti}_{2} \mathrm{O}_{7}$ grown in a oxygen atmosphere; with a small concentration of vacancies; we reproduce the field dependence of the ac susceptibility reported in [15]. Then, this result suggest that the small non-stoichiometry in $\mathrm{Gd}_{2} \mathrm{Ti}_{2} \mathrm{O}_{7}$ does not alter the field-driven phase transitions.

Results from the Curie-Weiss fit are shown in table 4.3 and 4.6 for $\mathrm{Gd}_{2} \mathrm{Ti}_{2} \mathrm{O}_{7}$ with annealings in oxygen and for $\mathrm{Gd}_{2} \mathrm{Ti}_{2} \mathrm{O}_{7}$ grown in different atmospheres respectively. We found that this liner fit for both batch provides $\mathrm{p}_{\text {eff }}$ in excellent agreement with the theoretical value of $\mu_{B} / \mathrm{Gd}$ ion. On the other side, the values of $\theta_{\mathrm{CW}}$ obtained for $\mathrm{Gd}_{2} \mathrm{Ti}_{2} \mathrm{O}_{7}$ with oxygen annealings are lower compared with the $\theta_{\mathrm{CW}}$ for $\mathrm{Gd}_{2} \mathrm{Ti}_{2} \mathrm{O}_{7}$ grown in different atmospheres. Also, all the values of $\theta_{\mathrm{CW}}$ turns out to be negative in agreement with the leading antiferromagnetic interaction present in the Hamiltonian of $\mathrm{Gd}_{2} \mathrm{Ti}_{2} \mathrm{O}_{7}$ [18]. The frustration index obtained suggest that when $\mathrm{Gd}_{2} \mathrm{Ti}_{2} \mathrm{O}_{7}$ is grown in different gaseous atmospheres such as oxygen and hydrogen the degree of frustration drops and the ordered 
ground state could be reached with a higher temperature.

Saturation magnetization of each batch display differences in the saturated moment. For example; as shown in figure 4.7; the $\mathrm{Gd}_{2} \mathrm{Ti}_{2} \mathrm{O}_{7}$ with a first and a second annealing in oxygen reaches saturated moments closer to the expected value $7 \mu_{B}$ in comparison to the as-grown sample. As shown in figure 4.14, for $\mathrm{Gd}_{2} \mathrm{Ti}_{2} \mathrm{O}_{7}$ grown in different atmospheres there is a very small difference between the saturated moments of $\mathrm{Gd}_{2} \mathrm{Ti}_{2} \mathrm{O}_{7}$ grown in air and oxygen. For $\mathrm{Gd}_{2} \mathrm{Ti}_{2} \mathrm{O}_{7}$ grown in hydrogen, the saturated moment reaches a value higher than $7 \mu_{B}$ which results of the incorrect use of the ideal stoichiometry to compute magnetization per mol. We first thought that this increase in the saturation magnetization to the magnetic moment of the $\mathrm{Ti}^{3+}$ present in oxygen-deficient pyrochlores, but as reported in [63] this contribution does not significantly affect the magnetization. For the two batch, the reduction in the saturated moment is related to the presence of oxygen vacancies in our $\mathrm{Gd}_{2} \mathrm{Ti}_{2} \mathrm{O}_{7}$ samples which is in agreement with magnetization measurements of the spin ice $\mathrm{Dy}_{2} \mathrm{Ti}_{2} \mathrm{O}_{7}$ reported in [63].

The magnetic specific heat $C_{m}$ of $\mathrm{Gd}_{2} \mathrm{Ti}_{2} \mathrm{O}_{7}$ presented in figure 4.15 shows that the two phase transitions occur at $\mathrm{T}_{\mathrm{N}}^{1}=1.04 \mathrm{~K}$ and $\mathrm{T}_{\mathrm{N}}^{2}=0.73 \mathrm{~K}$ consistent with the transitions temperatures of the literature $[39,51]$. As we report earlier, our $\mathrm{Gd}_{2} \mathrm{Ti}_{2} \mathrm{O}_{7}$ sample presents a slight deficiency of oxygen. This small non-stoichiometry seems not to alter the transition temperature. On the other hand, the recovered magnetic entropy at $10 \mathrm{~K}$ presents a reduction of $15 \%$ about the expected $\mathrm{R}$ $\ln (8)$ which we presume is associated to the oxygen vacancies. In order to confirm that, it is required to measure the magnetic specific heat $C_{m}$ below the lowest temperature $\sim 0.39 \mathrm{~K}$ considered here. Then, we could determine the cause of the reduction on the recovered entropy as due to the presence of oxygen vacancies or to the lack of measurements in lower temperatures. Also, it is important to study $\mathrm{Gd}_{2} \mathrm{Ti}_{2} \mathrm{O}_{7}$ samples with an appreciable deficiency of oxygen in order to see clear changes in the temperature transitions of specific heat and in the value of the recovered magnetic entropy at high temperatures.

For the magnetic specific heat $C_{m}$ in the low-temperature range studied, it is difficult to distinguish between the $\mathrm{T}^{2}$ and $\mathrm{T}^{3}$ power law as shown in figure 4.16. In addition, both fits result in values for the coefficients $\mathrm{A}$ and $\Lambda$ that are very close. As we mention before the $\mathrm{T}^{2}$ power law is proposed at low temperatures for $\mathrm{Gd}_{2} \mathrm{Ti}_{2} \mathrm{O}_{7}$ however the study of the $\mathrm{T}^{3}$ power law provides interesting results.In very simple terms, we notice that the developed theory for the antiferromagnet $\mathrm{Er}_{2} \mathrm{Ti}_{2} \mathrm{O}_{7}$ in [83] considers an appropriate dispersion relation for the spin-wave modes and also in the frame of spin-wave theory that the components of the velocity are approximately proportional to exchange interactions J. Then, 
it is computed an expression for the specific heat with a $\mathrm{T}^{3}$ dependence; equation (41); with the coefficient $\Lambda$ depending of the velocity of magnons. Within the $\mathrm{T}^{3}$ power law for the magnetic specific heat $C_{m}$, the magnons velocity in $\mathrm{Gd}_{2} \mathrm{Ti}_{2} \mathrm{O}_{7}$ was found to be $v_{s w}=46.2 \mathrm{~m} / \mathrm{s}$. This value of $v_{s w}$ is close to the magnons velocity reported in another pyrochlore antiferromagnets as $\mathrm{Yb}_{2} \mathrm{Ge}_{2} \mathrm{O}_{7}$ with transition temperature $\mathrm{T}_{\mathrm{N}}=0.62 \mathrm{~K}$ which is very close to $\mathrm{T}_{\mathrm{N}}^{2}$ resulting in $v_{s w}=45.8 \mathrm{~m} / \mathrm{s}$ [82], $\mathrm{Er}_{2} \mathrm{Ti}_{2} \mathrm{O}_{7}$ with $\mathrm{T}_{\mathrm{N}}=1.17 \mathrm{~K}$ and $v_{s w}=86 \mathrm{~m} / \mathrm{s}$ [11], and $\mathrm{Er}_{2} \mathrm{Ge}_{2} \mathrm{O}_{7}$ with $\mathrm{T}_{\mathrm{N}}=1.41 \mathrm{~K}$ and $v_{s w}=$ $132 \mathrm{~m} / \mathrm{s}$ [82]. Among these three examples, the spin-waves in $\mathrm{Gd}_{2} \mathrm{Ti}_{2} \mathrm{O}_{7}$ seems to behave in a similar way to $\mathrm{Yb}_{2} \mathrm{Ge}_{2} \mathrm{O}_{7}$. Also, we notice that an increase in the values of $v_{s w}$ is associated to an increase in the ordering temperature $\mathrm{T}_{\mathrm{N}}$.

The nearest-neighbour exchange interaction $\mathrm{J}$ can be estimated from the Curie-Weiss constant $\theta_{\mathrm{CW}}=-10.9 \mathrm{~K}$ by $\mathrm{J}=3 \theta_{\mathrm{CW}} / z J(J+1)=-0.35 \mathrm{~K}$, where $z=6$ is the number of nearest neighbours. On the other side, for a typical antiferromagnet the dispersion relation is given by $\omega=2 \mathrm{~J} S a k / \hbar=v_{s w} k$, where $S$ is the spin, $a$ is the lattice parameter, and $k$ is the wave vector [13]. Then, for $\mathrm{Gd}_{2} \mathrm{Ti}_{2} \mathrm{O}_{7}$ with $v_{s w}=46.2 \mathrm{~m} / \mathrm{s}$ the exchange interaction is $\mathrm{J} \sim-0.05 \mathrm{~K}$. This value of $0.05 \mathrm{~K}$ differs significantly from the previous $-0.35 \mathrm{~K}$. However, we think that this difference makes sense since it is not correct to suppose $\mathrm{Gd}_{2} \mathrm{Ti}_{2} \mathrm{O}_{7}$ with ordered spins since we know that below the second transition $1 / 4$ of the spins are still disordered in a dynamic state.

As shown in figure 4.17, the calculated fits to the Schottky anomaly does not described well the $C_{m}$ data of $\mathrm{Gd}_{2} \mathrm{Ti}_{2} \mathrm{O}_{7}$ in high magnetic fields. In fact, we expect this result since $\mathrm{Gd}_{2} \mathrm{Ti}_{2} \mathrm{O}_{7}$ with spin $S=7 / 2$ can no be studied as a system with two energy levels. A first fit to $C_{m}$ was reported by Raju et al. in [18] as shown in figure 2.14. This fit describes the Schottky peak by reaching also the peak of the first phase transition which we think that is still an arguable result. Another attempt to fit the $C_{m}$ data could be done by considering not only the system with the energy levels of the ${ }^{8} S_{7 / 2}$ state but also the split of the energy levels due to the crystal field as reported by [46] which we think that turns out to be a complicated problem. In addition, we found that the broad peaks of Schottky for our polycrystalline $\mathrm{Gd}_{2} \mathrm{Ti}_{2} \mathrm{O}_{7}$ are consistent with the specific heat measurements reported in [81] for single crystals. 


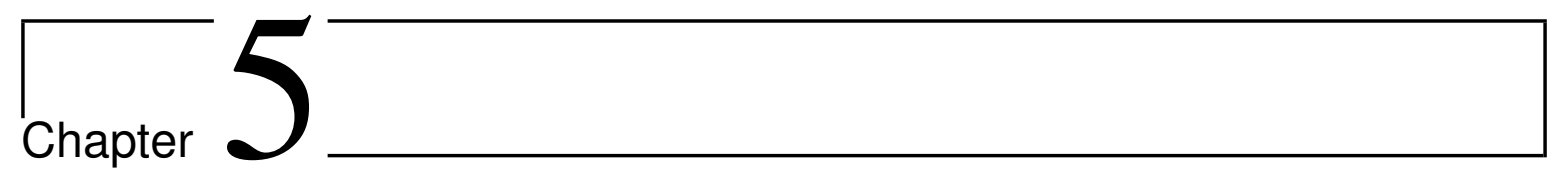

\section{Yttrium Dilution Effects on $\mathrm{Gd}_{2} \mathrm{Ti}_{2} \mathrm{O}_{7}$}

In Chapter 5 we complete our study of the structural defects in $\mathrm{Gd}_{2} \mathrm{Ti}_{2} \mathrm{O}_{7}$ by introducing non-magnetic yttrium $\mathrm{Y}$ on the gadolinium Gd sites of the pyrochlore lattice. This chapter covers the results of the structural and low-temperature magnetic properties of the yttrium diluted $\operatorname{Gd}_{2-\mathrm{x}} \mathrm{Y}_{\mathrm{x}} \operatorname{Ti}_{2} \mathrm{O}_{7}$. Thus, we discuss the trend and variation of these properties close to the first transition temperature $\mathrm{T}_{\mathrm{N}}^{1} \sim 1 \mathrm{~K}$ of $\mathrm{Gd}_{2} \mathrm{Ti}_{2} \mathrm{O}_{7}$ as we increase the yttrium content $\mathrm{x}$.

\section{$5.1 \quad$ Results}

We now study the low-temperature magnetic behaviour of the yttrium diluted $\operatorname{Gd}_{2-\mathrm{x}} \mathrm{Y}_{\mathrm{x}} \operatorname{Ti}_{2} \mathrm{O}_{7}$. Polycrystalline $\mathrm{Gd}_{2-\mathrm{x}} \mathrm{Y}_{\mathrm{x}} \mathrm{Ti}_{2} \mathrm{O}_{7}$ samples with different yttrium contents $\mathrm{x}$ were prepared by the sol-gel method described in Chapter 3. Here, a value of $\mathrm{x}=0.1$ means that the $\mathrm{Gd}_{2-\mathrm{x}} \mathrm{Y}_{\mathrm{x}} \mathrm{Ti}_{2} \mathrm{O}_{7}$ sample presents a $5 \%$ of mol substitution of the Gd sites by $\mathrm{Y}$, whereas for $\mathrm{x}=1$ there is a $50 \%$ of mol substitution of Gd by $\mathrm{Y}$. To reduce the oxygen deficiency, all the $\mathrm{Gd}_{2-\mathrm{x}} \mathrm{Y}_{\mathrm{x}} \mathrm{Ti}_{2} \mathrm{O}_{7}$ samples were annealed in oxygen flow $(50 \mathrm{ml} / \mathrm{min})$ at $1100^{\circ} \mathrm{C}$ for 24 hours. Then, in this section we first present the powder $\mathrm{X}$-ray diffraction (XRD) patterns of the $\mathrm{Gd}_{2-\mathrm{x}} \mathrm{Y}_{\mathrm{x}} \mathrm{Ti}_{2} \mathrm{O}_{7}$ samples and their respective refinement of the data by using the software FullProf. Also, in order to discuss the magnetic and thermal properties in $\mathrm{Gd}_{2-\mathrm{x}} \mathrm{Y}_{\mathrm{x}} \mathrm{Ti}_{2} \mathrm{O}_{7}$ we report ac susceptibility, magnetization, and specific heat data. 


\subsubsection{Structural analysis}

The powder X-ray diffraction patterns of the samples in the series $\mathrm{Gd}_{2-\mathrm{x}} \mathrm{Y}_{\mathrm{x}} \mathrm{Ti}_{2} \mathrm{O}_{7}(\mathrm{x}=0.1,0.14,0.2$, $0.3,0.4,0.7$ and 1.1) are shown in figure 5.1. It also displays the XRD pattern of the non-diluted $\mathrm{Gd}_{2} \mathrm{Ti}_{2} \mathrm{O}_{7}$. As the yttrium content $\mathrm{x}$ is increased, there is a very slight shift to the left of the peak centers due to differences in lattice parameters.

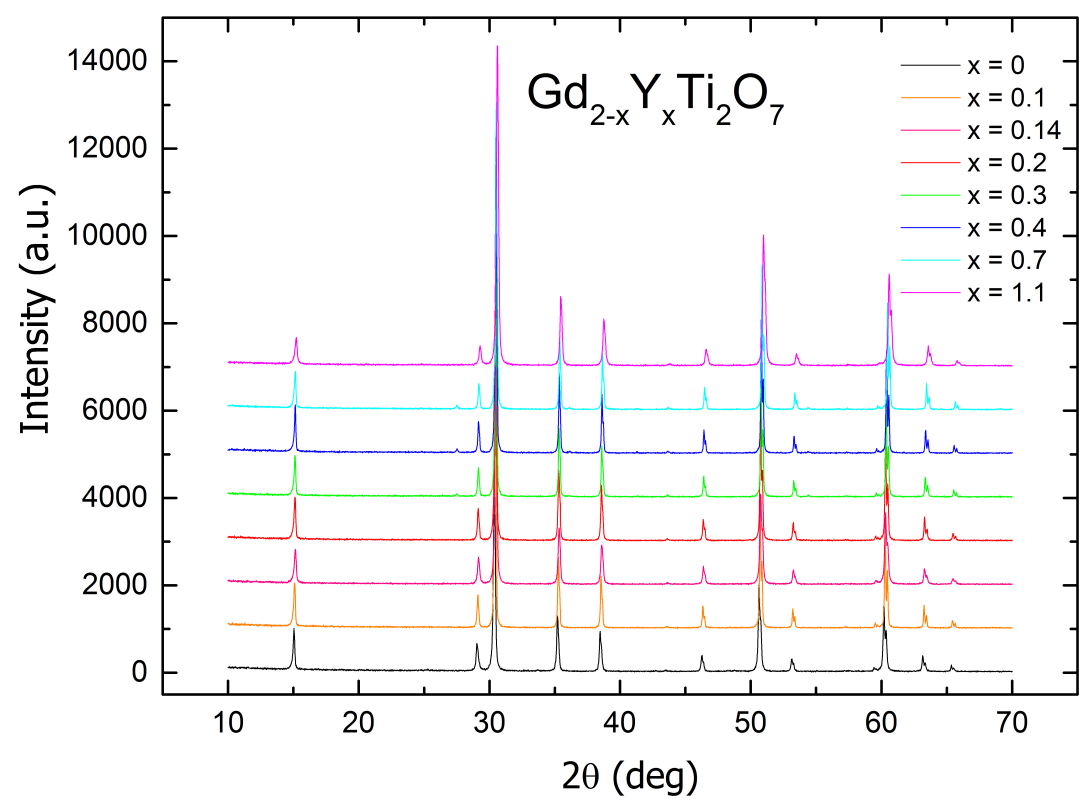

Figure 5.1: Powder X-ray diffraction patterns at room temperature, obtained from $\mathrm{Gd}_{2-\mathrm{x}} \mathrm{Y}_{\mathrm{x}} \mathrm{Ti}_{2} \mathrm{O}_{7}$ samples with different yttrium contents $\mathrm{x}$. The diffraction data is offset for clarity.

Rietveld refinements were carried out using the software FullProf and the graphical interface WinPLOTR. The results of the refinement are given in table 5.1. We found that all of our X-ray diffraction data refine in the pyrochlore structure with space group $F d \overline{3} m$. Even for a high yttrium content that is $55 \%$ of mol substitution $(\mathrm{x}=1.1)$ the average structure becomes pyrochlore. We also found that the refined lattice parameter decreases from 10.184 to $10.140 \AA$ as the yttrium content $\mathrm{x}$ increases in $\mathrm{Gd}_{2-\mathrm{x}} \mathrm{Y}_{\mathrm{x}} \mathrm{Ti}_{2} \mathrm{O}_{7}$. Figure 5.2 displays this reduction on the lattice parameter which can be attributed to the smaller ionic radius of $\mathrm{Y}^{3+}$. From the refinement, we obtained that the occupancies of the Gd sites are very close to one showing that there are no vacant sites of Gd even if the Gd content decreases in $\mathrm{Gd}_{2-\mathrm{x}} \mathrm{Y}_{\mathrm{x}} \mathrm{Ti}_{2} \mathrm{O}_{7}$. On the other hand, the refinement reveals that the occupancies of the $\mathrm{Y}$ sites are very close to one for small values of $\mathrm{Y}$ content. The occupancies for $\mathrm{x}=0.7$ and 1.1 seems to 
CHAPTER 5. Yttrium Dilution Effects on $\mathrm{Gd}_{2} \mathrm{Ti}_{2} \mathrm{O}_{7}$

show that as we increase the $\mathrm{Y}$ content it is more likely find vacancies on the $\mathrm{Y}$ sites. The occupancies of $\mathrm{Ti}$ have values very close to one showing that there is no a titanium deficiency. Once again, the vacancies were found to be on the $\mathrm{O}(1)$ sites for all the yttrium diluted samples. Furthermore, for all the diluted samples studied here, the values of the occupancies $\mathrm{O}(1)$ do not present a significant difference between them. For the $\mathrm{O}(2)$ sites, the values of occupancies are close to one suggesting no oxygen vacancies as in our previous results of the non-diluted $\mathrm{Gd}_{2} \mathrm{Ti}_{2} \mathrm{O}_{7}$. We refined all data sets in order to obtain the lowest chi-squared $\chi^{2}$ which reach minimums around a value of two.

\begin{tabular}{c|c|c|c|c|c|c|c|c|c}
\hline Yttrium content $\mathrm{x}$ & 0 & 0.1 & 0.14 & 0.2 & 0.3 & 0.4 & 0.7 & 1.1 \\
\hline \hline Space group & \multicolumn{7}{|c|}{$F d \overline{3} m$} \\
\hline Lattice parameter $(\AA)$ & 10.184 & 10.176 & 10.176 & 10.175 & 10.165 & 10.163 & 10.148 & 10.140 \\
\hline $\mathrm{Gd}$ & 1.03 & 0.95 & 1 & 1 & 1 & 1 & 1 & 1 \\
\hline $\mathrm{Y}$ & 0 & 0.98 & 1.03 & 0.99 & 0.98 & 1.03 & 0.82 & 0.90 \\
\hline $\mathrm{Ti}$ & 1.05 & 1 & 1.04 & 1.05 & 1.06 & 1.05 & 1 & 1 \\
\hline $\mathrm{O}(1)$ & 0.66 & 0.77 & 0.74 & 0.75 & 0.81 & 0.77 & 0.80 & 0.73 \\
\hline $\mathrm{O}(2)$ & 0.98 & 0.92 & 0.96 & 0.97 & 0.96 & 0.95 & 0.92 & 0.93 \\
\hline$\chi^{2}$ & 2.09 & 2.11 & 2.02 & 2.09 & 2.14 & 2.53 & 2.33 & 2.12 \\
\hline \hline
\end{tabular}

Table 5.1: Refinement of the structure of $\mathrm{Gd}_{2-\mathrm{x}} \mathrm{Y}_{\mathrm{x}} \mathrm{Ti}_{2} \mathrm{O}_{7}$ with different yttrium contents $\mathrm{x}$.

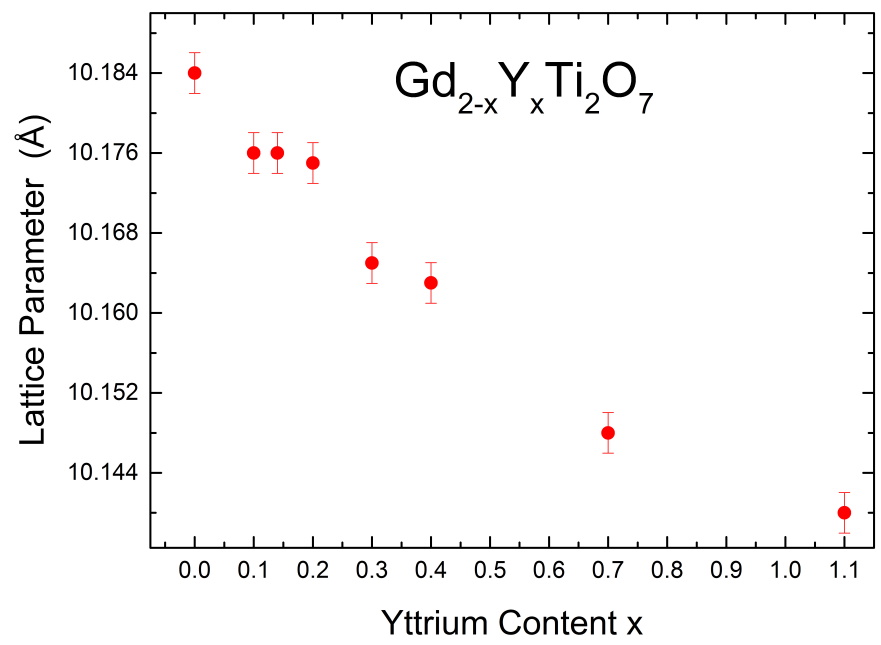

Figure 5.2: Refined lattice parameter $( \pm 0.02 \%)$ as a function of the yttrium content $\mathrm{x}$. 


\subsubsection{AC magnetic susceptibility}

AC magnetic susceptibility measurements were conducted on needle-shaped samples for $\mathrm{Gd}_{2-\mathrm{x}} \mathrm{Y}_{\mathrm{x}} \mathrm{Ti}_{2} \mathrm{O}_{7}$ with different yttrium concentrations. Above $4.2 \mathrm{~K}$, the ac magnetic susceptibility $\chi_{a c}$ was measured using the SQUID. Below $4.2 \mathrm{~K}, \chi_{a c}$ was measured on the ac susceptometer using a mutual inductance bridge at frequency $f=155 \mathrm{~Hz}$ and modulation field $\mu_{0} h_{a c}=10 \mathrm{mT}$.

In figure 5.3 we present measurements of $\chi_{a c}$ as a function of the temperature for $\operatorname{Gd}_{2-\mathrm{x}} \mathrm{Y}_{\mathrm{x}} \mathrm{Ti}_{2} \mathrm{O}_{7}$ with different yttrium contents $\mathrm{x}$. The $\chi_{a c}$ data is presented in the temperature range of 0.4 to $7 \mathrm{~K}$ and was collected at zero field. In each $\mathrm{Gd}_{2-\mathrm{x}} \mathrm{Y}_{\mathrm{x}} \mathrm{Ti}_{2} \mathrm{O}_{7}$ compound, the ac susceptibility exhibits an anomaly close below $1 \mathrm{~K}$ which corresponds to the first phase transition. For $\operatorname{Gd}_{2-\mathrm{x}} \mathrm{Y}_{\mathrm{x}} \operatorname{Ti}_{2} \mathrm{O}_{7}$ samples with small yttrium content or $\mathrm{x}=0.1,0.14,0.16$ and 0.2 , the values of susceptibility $\chi_{a c}$ remain almost constant for each sample after the peak at its respective transition temperature $\mathrm{T}_{\mathrm{N}}^{1}$. Also, for a slightly higher yttrium content that is for $\mathrm{x}=0.3$ and 0.4 we observe that the values of $\chi_{a c}$ fall short after the phase transition at $\mathrm{T}_{\mathrm{N}}^{1}=0.74$ and $0.67 \mathrm{~K}$ respectively. Then, for high yttrium content: $\mathrm{x}=0.7$ and 1 , it is clear that after the phase transition the values of $\chi_{a c}$ start to fall in a high rate defining well the peaks. The collected data of susceptibility does not show a second phase transition for any of our yttrium diluted $\mathrm{Gd}_{2-\mathrm{x}} \mathrm{Y}_{\mathrm{x}} \mathrm{Ti}_{2} \mathrm{O}_{7}$ samples.

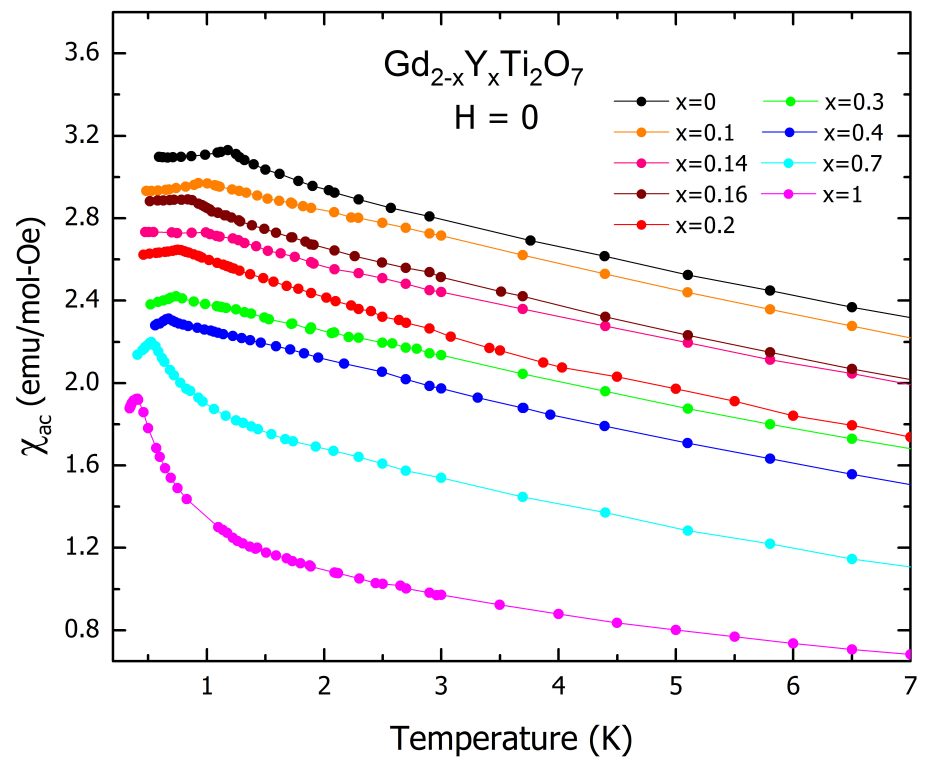

Figure 5.3: ac magnetic susceptibility $\chi_{a c}$ as a function of the temperature for $\operatorname{Gd}_{2-\mathrm{x}} \mathrm{Y}_{\mathrm{x}} \operatorname{Ti}_{2} \mathrm{O}_{7}$ with different yttrium contents $\mathrm{x}$ measured at zero field. $\chi_{a c}$ curves are slight offset for clarity. 
The left side of figure 5.4 shows the $\chi_{a c}$ curves in a larger temperature range with at zero field. It illustrates better the peaks and indicates a progressive decrease in the transition temperature $\mathrm{T}_{\mathrm{N}}^{1}$. For high yttrium contents: $\mathrm{x}=0.7$ and 1 , we have that the susceptibility curves increase in a very high rate close to the anomaly compared with the samples with $\mathrm{x}<4$ and also display a more defined peak. The right side of figure 5.4 displays the raw data of $\chi_{a c}$. It shows that as we increase the yttrium content $\mathrm{x}$ in $\mathrm{Gd}_{2-\mathrm{x}} \mathrm{Y}_{\mathrm{x}} \mathrm{Ti}_{2} \mathrm{O}_{7}$, the values of $\chi_{a c}$ decrease and also that the position of the peaks at $\mathrm{T}_{\mathrm{N}}^{1}$ shift slightly to the left. For $\mathrm{x}=0.1,0.14,0.16$, and $0.2 \chi_{a c}$ curves converge with an analogue behaviour in the temperature interval of 5 to $30 \mathrm{~K}$. Also, for $\mathrm{x}=0.3,0.4$, and 0.7 the $\chi_{a c}$ data seems to approach the anomaly with the same behaviour. We found that the $\chi_{a c}$ curve of the sample with $\mathrm{x}=1$ presents a very broad kink before reaching the temperature of the sharp peak. This is a very different behaviour compared with the smooth growing of the $\chi_{a c}$ curves for lower yttrium contents.
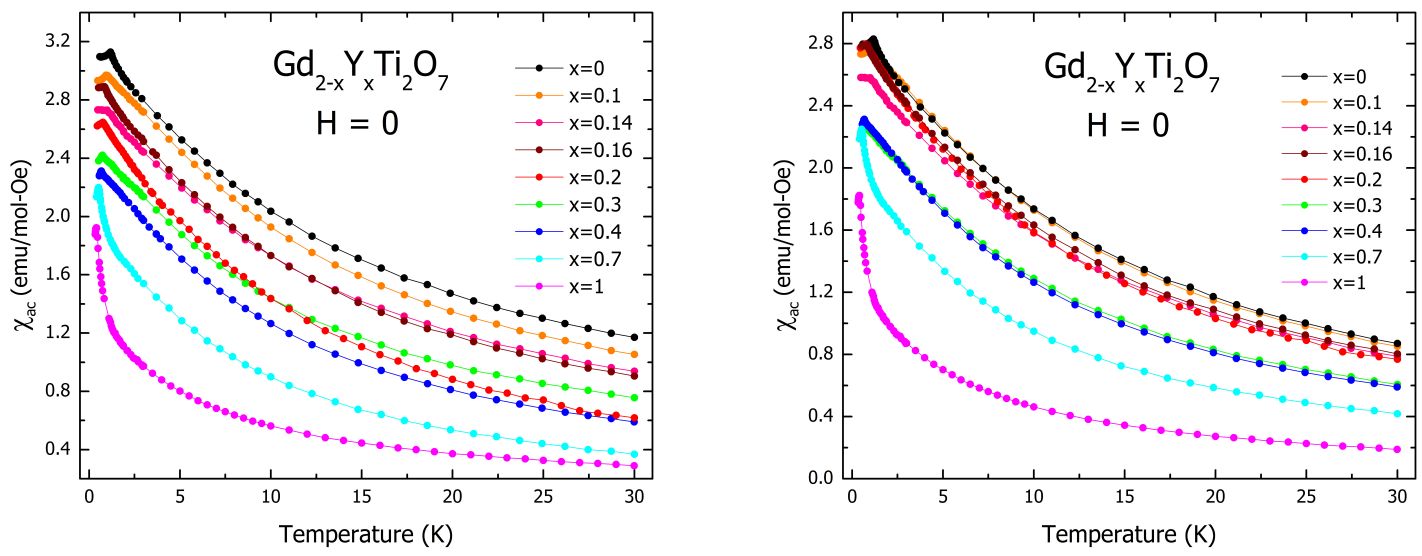

Figure 5.4: Left: Temperature dependence of the ac magnetic susceptibility $\chi_{a c}$ of $\operatorname{Gd}_{2-\mathrm{x}} \mathrm{Y}_{\mathrm{x}} \operatorname{Ti}_{2} \mathrm{O}_{7}$ for $\mathrm{x}=0-0.7$. The $\chi_{a c}$ curves are slightly offset for clarity. Right: Raw data for the ac magnetic susceptibility of $\mathrm{Gd}_{2-\mathrm{x}} \mathrm{Y}_{\mathrm{x}} \mathrm{Ti}_{2} \mathrm{O}_{7}$.

From the ac susceptibility data, we report the transition temperatures $\mathrm{T}_{\mathrm{N}}^{1}$ in table 5.2. The values of $\mathrm{T}_{\mathrm{N}}^{1}$ in fact decrease from $1.18 \mathrm{~K}$ for $\mathrm{x}=0$ to $0.41 \mathrm{~K}$ for $\mathrm{x}=1$ when the yttrium content $\mathrm{x}$ is increased. It implies that the degree of geometric frustration rises with the yttrium dilution in the lattice and also that the antiferromagnetic interactions between Gd spins, in first approximation, require lower temperatures to develop a long-range order. We did not collect $\chi_{a c}$ data for yttrium contents $\mathrm{x}$ higher than 1 since for $\mathrm{x}=1$ we have an ordering temperature $\mathrm{T}_{\mathrm{N}}^{1}=0.41 \mathrm{~K}$ which is closer to the minimum reached in the laboratory, $0.39 \mathrm{~K}$. So, for values $\mathrm{x}>1$ it is not possible to resolve 
CHAPTER 5. Yttrium Dilution Effects on $\mathrm{Gd}_{2} \mathrm{Ti}_{2} \mathrm{O}_{7}$

the peak of the first phase transition.

\begin{tabular}{c|c|c}
\hline $\mathrm{Gd}_{2-\mathrm{x}} \mathrm{Y}_{\mathrm{x}} \mathrm{Ti}_{2} \mathrm{O}_{7}$ & Yttrium content $\mathrm{x}$ & $\mathrm{T}_{\mathrm{N}}^{1}(\mathrm{~K})$ \\
\hline \hline $\mathrm{Gd}_{2} \mathrm{Ti}_{2} \mathrm{O}_{7}$ & 0 & 1.18 \\
\hline $\mathrm{Gd}_{1.9} \mathrm{Y}_{0.1} \mathrm{Ti}_{2} \mathrm{O}_{7}$ & 0.1 & 0.97 \\
\hline $\mathrm{Gd}_{1.86} \mathrm{Y}_{0.14} \mathrm{Ti}_{2} \mathrm{O}_{7}$ & 0.14 & 0.92 \\
\hline $\mathrm{Gd}_{1.8} \mathrm{Y}_{0.2} \mathrm{Ti}_{2} \mathrm{O}_{7}$ & 0.2 & 0.77 \\
\hline $\mathrm{Gd}_{1.7} \mathrm{Y}_{0.3} \mathrm{Ti}_{2} \mathrm{O}_{7}$ & 0.3 & 0.74 \\
\hline $\mathrm{Gd}_{1.6} \mathrm{Y}_{0.4} \mathrm{Ti}_{2} \mathrm{O}_{7}$ & 0.4 & 0.67 \\
\hline $\mathrm{Gd}_{1.3} \mathrm{Y}_{0.7} \mathrm{Ti}_{2} \mathrm{O}_{7}$ & 0.7 & 0.54 \\
\hline $\mathrm{Gd}_{1} \mathrm{Y}_{1} \mathrm{Ti}_{2} \mathrm{O}_{7}$ & 1 & 0.41 \\
\hline \hline
\end{tabular}

Table 5.2: Values of the transition temperatures $\mathrm{T}_{\mathrm{N}}^{1}$ for the yttrium diluted $\mathrm{Gd}_{2-\mathrm{x}} \mathrm{Y}_{\mathrm{x}} \operatorname{Ti}_{2} \mathrm{O}_{7}$.

Figure 5.5 displays the transition temperatures $\mathrm{T}_{\mathrm{N}}^{1}$ as a function of the yttrium content $\mathrm{x}$ in $\mathrm{Gd}_{2-\mathrm{x}} \mathrm{Y}_{\mathrm{x}} \mathrm{Ti}_{2} \mathrm{O}_{7}$. We observe that for small yttrium contents the ordering temperatures experiments a quick variation until $\mathrm{x}=0.2$ or 0.3 where it stabilizes. Then, for a higher concentration of yttrium that is for $\mathrm{x}>0.3$, we notice the linear dependence of the transition temperatures $\mathrm{T}_{\mathrm{N}}^{1}$.

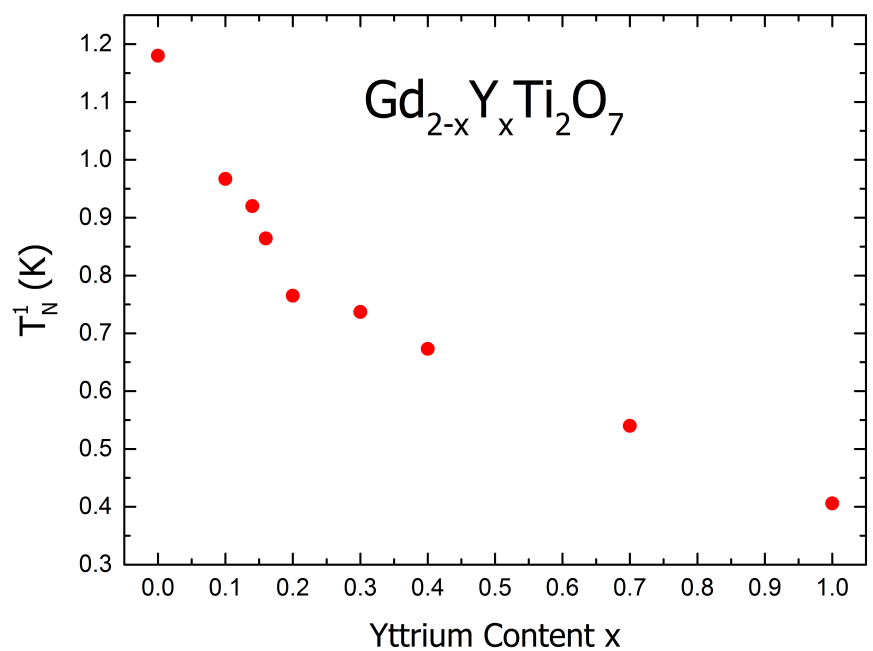

Figure 5.5: Transition temperatures $\mathrm{T}_{\mathrm{N}}^{1}$ as a function of the yttrium content $\mathrm{x}$ in $\mathrm{Gd}_{2-\mathrm{x}} \mathrm{Y}_{\mathrm{x}} \mathrm{Ti}_{2} \mathrm{O}_{7}$. 
Now we study if field-induced phase transitions occur for a diluted sample with yttrium content $\mathrm{x}=0.3$ or $\mathrm{Gd}_{1.7} \mathrm{Y}_{0.3} \mathrm{Ti}_{2} \mathrm{O}_{7}$. From our previous results, we report that for $\mathrm{x}=0.3$ the diluted sample undergoes a phase transition at a temperature $\mathrm{T}_{\mathrm{N}}^{1}=0.72 \mathrm{~K}$. In figure 5.6 we present the ac susceptibility data as a function of the applied field, $\chi_{a c}(\mathrm{H})$, for $\mathrm{x}=0.3$. Then, the $\chi_{a c}(\mathrm{H})$ data shows that there is no peak indicating phase transition at temperatures 0.63 and $0.51 \mathrm{~K}$, and for field values around $7 \mathrm{~T}$ the susceptibility displays a broad downturn and goes to zero. We think that there is no phase transition at 0.63 and $0.51 \mathrm{~K}$ as the field increases because these temperatures are very close to the transition temperature $\mathrm{T}_{\mathrm{N}}^{1}=0.72 \mathrm{~K}$ and it is required a lower temperature to observe another phase transition. Also, $0.51 \mathrm{~K}$ is a temperature very close to the minimum reached in the lab: 0.49 $\mathrm{K}$, so $\chi_{a c}$ measurements for lower temperatures were not carried on.

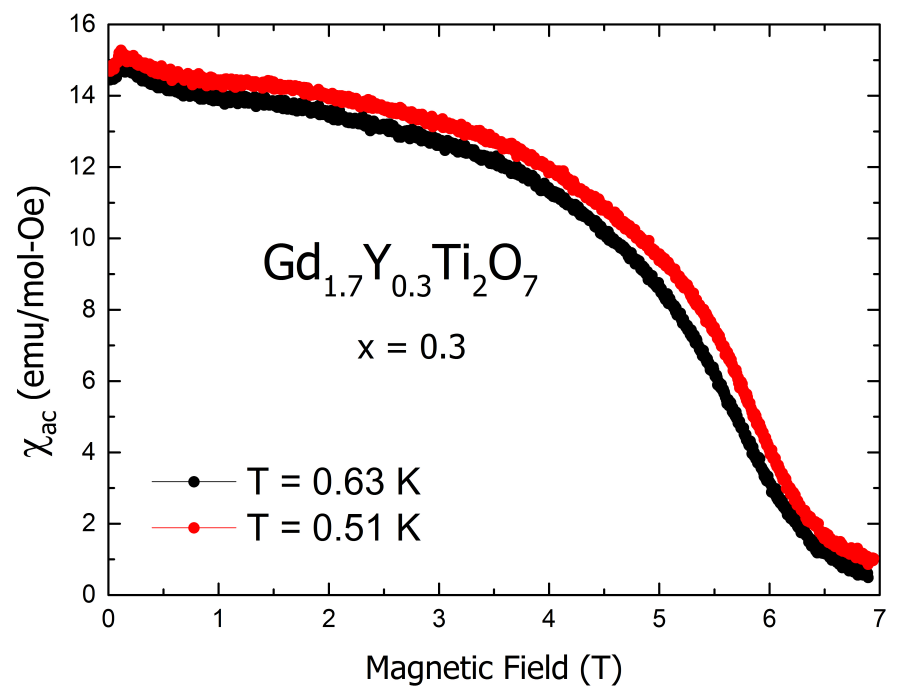

Figure 5.6: Field dependence of ac susceptibility for $\mathrm{Gd}_{2-\mathrm{x}} \mathrm{Y}_{\mathrm{x}} \mathrm{Ti}_{2} \mathrm{O}_{7}$ with yttrium content $\mathrm{x}=0.3$ measured at 0.63 and $0.51 \mathrm{~K}$. 


\subsubsection{DC magnetic susceptibility}

We present in figure 5.7 the temperature dependence of the inverse dc susceptibility $\chi^{-1}$ for $\mathrm{Gd}_{2-\mathrm{x}} \mathrm{Y}_{\mathrm{x}} \mathrm{Ti}_{2} \mathrm{O}_{7}$ with different yttrium contents $\mathrm{x}$. The $\chi^{-1}$ data was collected in the temperature range of 2 to 300 $\mathrm{K}$ and was measured in an applied field of $\mathrm{H}=100$ Oe. Figure 5.7 also displays the respective CurieWeiss linear fit in high temperatures for each $\mathrm{Gd}_{2-\mathrm{x}} \mathrm{Y}_{\mathrm{x}} \mathrm{Ti}_{2} \mathrm{O}_{7}$ sample. We notice that when the yttrium content is increased, the slope of the linear fit (inversely proportional to $\left|\theta_{\mathrm{CW}}\right|$ ) tends to increase which means a reduction in the antiferromagnetic exchange interaction between the Gd spins.

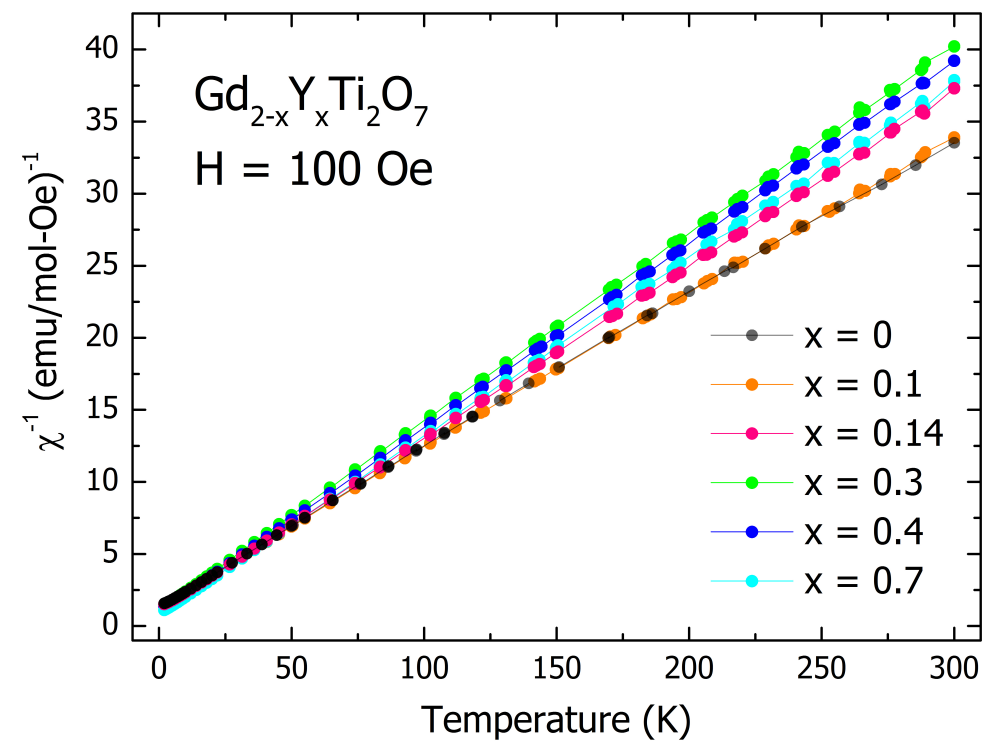

Figure 5.7: Inverse susceptibility $\chi^{-1}$ as a function of the temperature and its respective linear fit of Curie-Weiss for $\mathrm{Gd}_{2-\mathrm{x}} \mathrm{Y}_{\mathrm{x}} \mathrm{Ti}_{2} \mathrm{O}_{7}$ measured in an applied field of 100 Oe.

From the linear fit we extract the Curie-Weiss temperatures, $\theta_{\mathrm{CW}}$, and effective magnetic moments, $\mathrm{p}_{\text {eff }}$ and report these values in table 5.3. From the values of the transition temperatures $\mathrm{T}_{\mathrm{N}}^{1}$, we obtain the frustration index $f$. For small yttrium contents in $\mathrm{Gd}_{2-\mathrm{x}} \mathrm{Y}_{\mathrm{x}} \mathrm{Ti}_{2} \mathrm{O}_{7}$ that is for $\mathrm{x}=0.1$ and 0.14 , the degree of frustration $f$ almost does not vary and stay below the reported frustration index of $\mathrm{Gd}_{2} \mathrm{Ti}_{2} \mathrm{O}_{7}[15], f \sim 10$. When the yttrium content $\mathrm{x}$ is increased to 0.2 and forward, the frustration index exceeds the index of $\mathrm{Gd}_{2} \mathrm{Ti}_{2} \mathrm{O}_{7}$ becoming evident the higher frustration of the system and that we will need lower temperatures to order the Gd spins. 
CHAPTER 5. Yttrium Dilution Effects on $\mathrm{Gd}_{2} \mathrm{Ti}_{2} \mathrm{O}_{7}$

\begin{tabular}{c|c|c|c|c|c|c|c|c}
\hline $\mathrm{x}$ & 0 & 0.1 & 0.14 & 0.2 & 0.3 & 0.4 & 0.7 & 1 \\
\hline \hline $\mathrm{p}_{\text {eff }}\left(\mu_{B} / \mathrm{Gd}^{3+}\right)$ & 7.9 & 7.9 & 8.1 & 8.1 & 7.9 & 8.0 & 7.6 & 8.0 \\
\hline$\theta_{\mathrm{CW}}(\mathrm{K})$ & -9.7 & -8.8 & -8.7 & -8.9 & -8.3 & -7.5 & -6.5 & -4.1 \\
\hline $\mathrm{T}_{\mathrm{N}}^{1}(\mathrm{~K})$ & 1.18 & 0.97 & 0.92 & 0.77 & 0.74 & 0.67 & 0.54 & 0.41 \\
\hline$f$ & 8.2 & 9.1 & 9.5 & 11.6 & 11.2 & 11.2 & 12.0 & 10 \\
\hline \hline
\end{tabular}

Table 5.3: Values of the effective magnetic moment, $\mathrm{p}_{e f f}$, Curie-Weiss temperatures, $\theta_{\mathrm{CW}}$, and transition temperatures, $\mathrm{T}_{\mathrm{N}}^{1}$, for different yttrium concentrations $\mathrm{x}$ in $\mathrm{Gd}_{2-\mathrm{x}} \mathrm{Y}_{\mathrm{x}} \mathrm{Ti}_{2} \mathrm{O}_{7}$.

Figure 5.8 presents the values of $\left|\theta_{\mathrm{CW}}\right|$ as a dependence of the yttrium content $\mathrm{x}$ in $\operatorname{Gd}_{2-\mathrm{x}} \mathrm{Y}_{\mathrm{x}} \operatorname{Ti}_{2} \mathrm{O}_{7}$. The values of $\left|\theta_{\mathrm{CW}}\right|$ decrease almost linearly from the value of $9.7 \mathrm{~K}$ of the non-diluted sample as the yttrium concentration rises in agreement with [18] meaning smaller antiferromagnetic exchanges interactions. This linear dependence as a function of the yttrium content $\mathrm{x}$ is a reminiscent of the linear tendency of the $\mathrm{T}_{\mathrm{N}}^{1}$ (figure 5.5). We think that more measurements with other different yttrium contents $\mathrm{x}$ will help to clarify the behaviour of the $\theta_{\mathrm{CW}}$. Also, figure 5.8 displays the values of the peff of $\mathrm{Gd}_{2-\mathrm{x}} \mathrm{Y}_{\mathrm{x}} \mathrm{Ti}_{2} \mathrm{O}_{7}$ as the yttrium content $\mathrm{x}$ is increased. It shows that the peff values of all our diluted samples oscillate around the expected value of $7.94 \mu_{B} / \mathrm{Gd}[9]$ without a tendency in particular and present slight differences between them as the yttrium concentration rises.

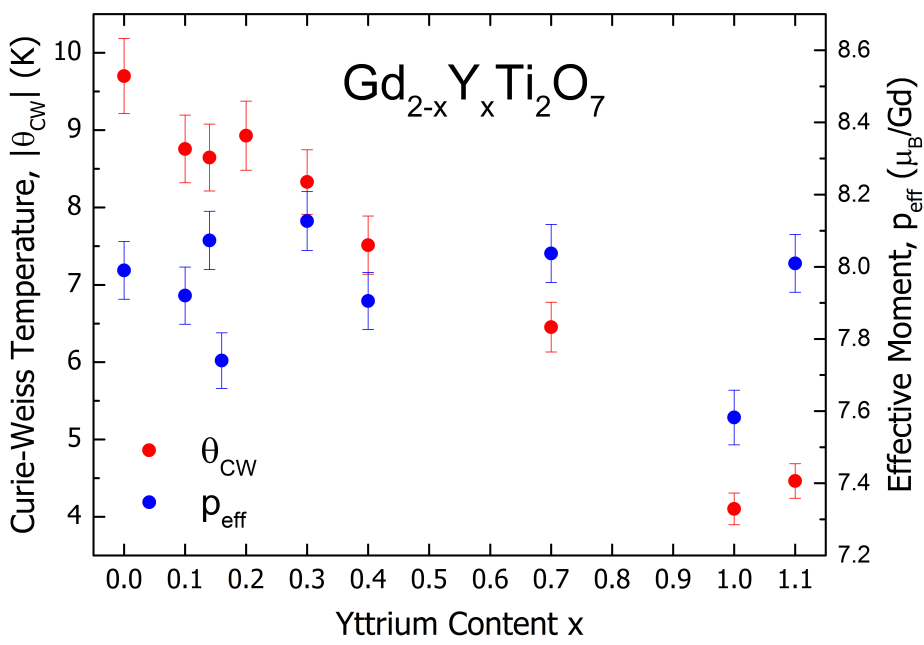

Figure 5.8: Curie-Weiss temperatures $\left|\theta_{\mathrm{CW}}\right|( \pm 5 \%)$ (left axis) and effective magnetic moments $\mathrm{p}_{\mathrm{eff}}$ $( \pm 1 \%)$ (right axis) for $\mathrm{Gd}_{2-\mathrm{x}} \mathrm{Y}_{\mathrm{x}} \mathrm{Ti}_{2} \mathrm{O}_{7}$ as a function of the yttrium content $\mathrm{x}$. 


\subsubsection{Saturation magnetization}

Measurements of magnetization of the yttrium-diluted $\mathrm{Gd}_{2-\mathrm{x}} \mathrm{Y}_{\mathrm{x}} \mathrm{Ti}_{2} \mathrm{O}_{7}$ samples were carried out on a vibrating sample magnetometer (VSM). Figure 5.9 shows the magnetization curves as a function of the applied field for $\mathrm{Gd}_{2-\mathrm{x}} \mathrm{Y}_{\mathrm{x}} \mathrm{Ti}_{2} \mathrm{O}_{7}$ with small values of yttrium content $\mathrm{x}$. At $4.2 \mathrm{~K}$, for $\mathrm{x}=0.2$ and 0.3 that is $\mathrm{Gd}_{1.8} \mathrm{Y}_{0.2} \mathrm{Ti}_{2} \mathrm{O}_{7}$ and $\mathrm{Gd}_{1.7} \mathrm{Y}_{0.3} \mathrm{Ti}_{2} \mathrm{O}_{7}$ respectively we observe the same curve behaviours for values of magnetic field up to $3 \mathrm{~T}$. As we increase the magnetic field those samples reach the expected saturated moment of $7 \mu_{B}$ but the magnetization of the sample with $\mathrm{x}=0.3$ saturates above $8 \mathrm{~T}$ in comparison with $\mathrm{x}=0.2$ which saturates for a higher field close to $14 \mathrm{~T}$. It seems that this yttrium content $\mathrm{x}=0.2$ marks a point for which it is needed very high fields in order to reach the expected moment. At $1.28 \mathrm{~K}$, we observe that for the samples with $\mathrm{x}=0.4$ and $\mathrm{x}=0.7$ the values of magnetization grow rapidly with the magnetic field and saturate around values of $7 \mathrm{~T}$. For $\mathrm{x}=$ 0.4 , we found a lower and unexpected saturation value which could be related to non-stoichiometric of this sample. We also show the saturation magnetization curve for the non-diluted sample $\mathrm{x}=0$ at $1.26 \mathrm{~K}$ in order to compare it with the different saturation curves of the diluted samples.

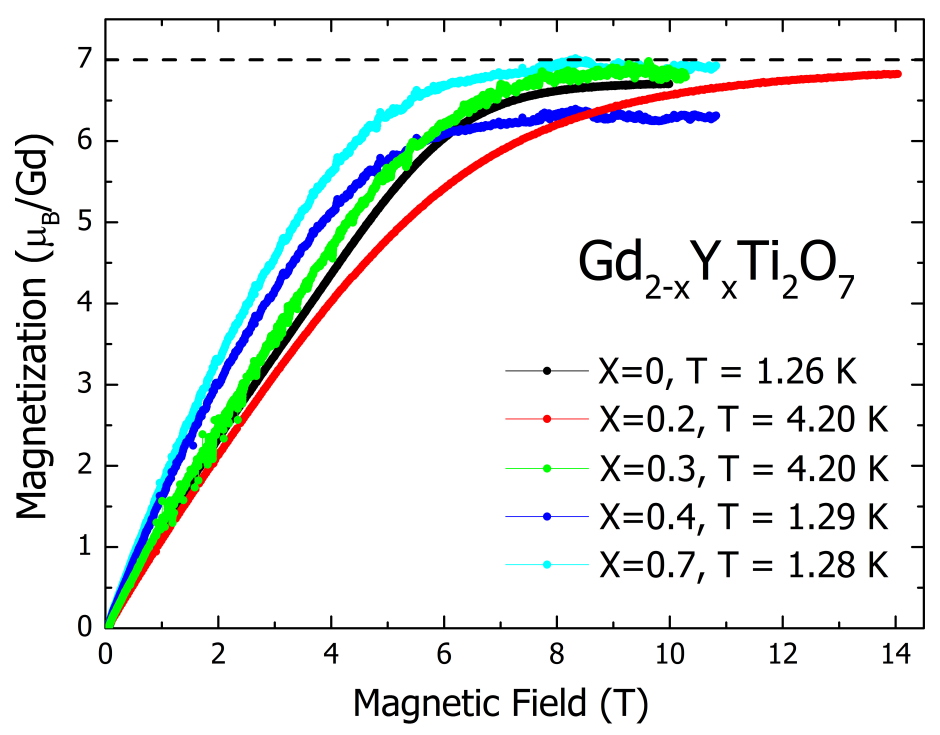

Figure 5.9: Saturation magnetization for $\mathrm{Gd}_{2-\mathrm{x}} \mathrm{Y}_{\mathrm{x}} \mathrm{Ti}_{2} \mathrm{O}_{7}$ with different yttrium concentrations $\mathrm{x}$ measured at 1.28 and $4.2 \mathrm{~K}$.

Having presented the saturation magnetization for small yttrium contents in $\operatorname{Gd}_{2-\mathrm{x}} \mathrm{Y}_{\mathrm{x}} \mathrm{Ti}_{2} \mathrm{O}_{7}$, now we report on figure 5.10 the magnetization curves for $\mathrm{x}=1,1.1$, and 1.5. The field dependence of the 
magnetization data was measured using a commercial SQUID at a fixed temperature $\mathrm{T}=4.2 \mathrm{~K}$. We found that as the applied field increases up to a value of $7 \mathrm{~T}$, the magnetization grows at a considerable rate, and reaches a value of $6.8 \mu_{B} / \mathrm{Gd}$ ion. Also, the behaviour of the magnetization curves suggest that for a higher yttrium content it is easy to reach the saturated moment. For values above $7 \mathrm{~T}$, we expect that the samples with high yttrium content reach the expected saturation moment of $7 \mu_{B}$.

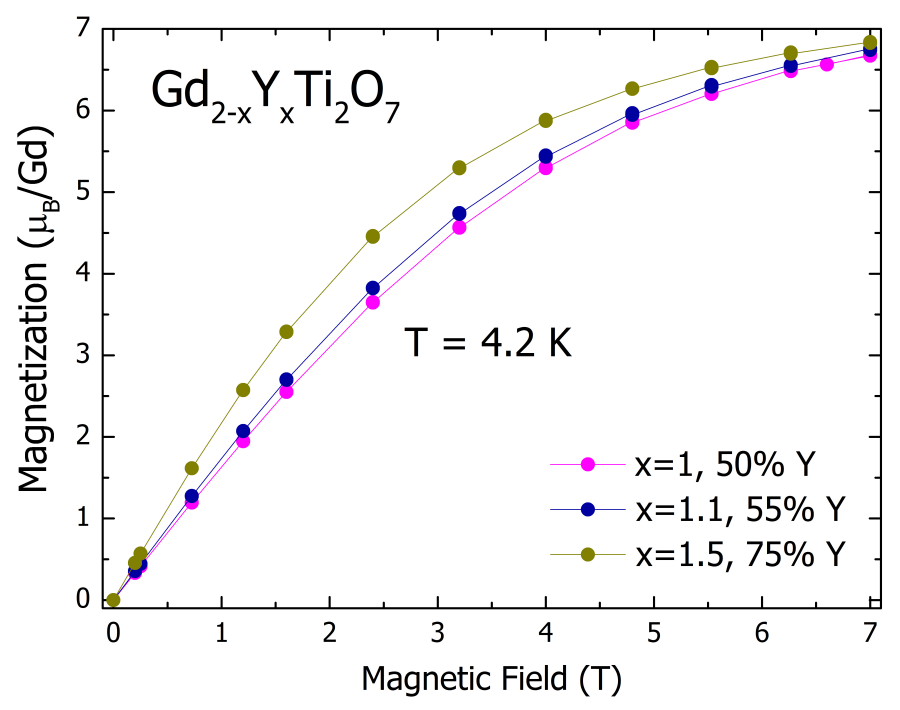

Figure 5.10: Magnetization as a function of the applied field of $\mathrm{Gd}_{2-\mathrm{x}} \mathrm{Y}_{\mathrm{x}} \operatorname{Ti}_{2} \mathrm{O}_{7}$ with $\mathrm{x}=1,1.1$ and 1.5 measured at $4.2 \mathrm{~K}$. 


\subsubsection{Specific heat}

The specific heat data for $\mathrm{Gd}_{2-\mathrm{x}} \mathrm{Y}_{\mathrm{x}} \mathrm{Ti}_{2} \mathrm{O}_{7}$ with different yttrium contents was collected using a Quantum Design PPMS calorimeter. Figure 5.11 displays the total specific heat $C$ as a function of the temperature for the yttrium diluted samples $\mathrm{Gd}_{2-\mathrm{x}} \mathrm{Y}_{\mathrm{x}} \mathrm{Ti}_{2} \mathrm{O}_{7}$ measured in zero field. Up to temperature of $50 \mathrm{~K}$, the $C$ data for $\mathrm{x}=0,0.1,0.2$, and 0.4 behave with a linear dependence and present very slight variations between them in contrast with the samples with a higher yttrium content such as $\mathrm{x}$ $=0.7$ and 1. In order to compute the magnetic contribution to the specific heat $C_{m}$ we examine $C$ in a lower temperature range. Between 10 and $15 \mathrm{~K}$, as shown by figure 5.11 , the $C$ curves for all the different yttrium contents are very close to each other and behave in a similar way. So, we expect that the lattice specific heat $C_{\text {lat }}$ for the $\mathrm{Gd}_{2-\mathrm{x}} \mathrm{Y}_{\mathrm{x}} \mathrm{Ti}_{2} \mathrm{O}_{7}$ samples to be the same as the estimated $C_{\text {lat }}$ of $\mathrm{Gd}_{2} \mathrm{Ti}_{2} \mathrm{O}_{7}$ obtained in Chapter 4 from a $\mathrm{T}^{3}$ fit. Then, the magnetic specific heat $C_{m}$ is obtained by subtracting $C_{l a t}$ from $C$ and its temperature variation is shown in the figures below.

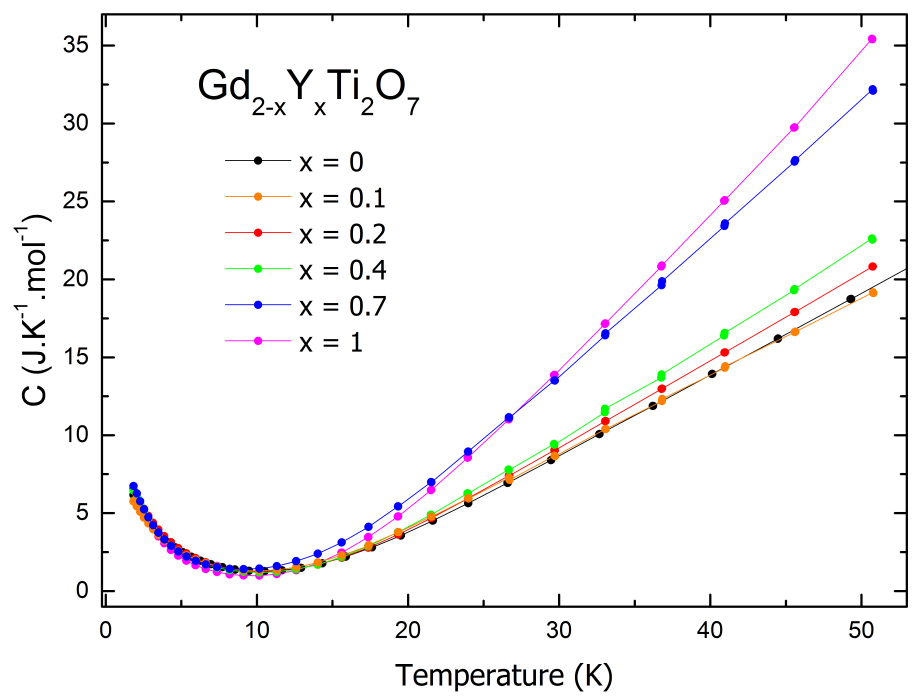

Figure 5.11: Temperature dependence of the total specific heat $C$ for $\mathrm{Gd}_{2-\mathrm{x}} \mathrm{Y}_{\mathrm{x}} \mathrm{Ti}_{2} \mathrm{O}_{7}$ with different yttrium contents $\mathrm{x}$ measured in zero field.

Figure 5.12 shows the temperature and field dependencies of the magnetic specific heat $C_{m}$ for $\mathrm{Gd}_{2-\mathrm{x}} \mathrm{Y}_{\mathrm{x}} \mathrm{Ti}_{2} \mathrm{O}_{7}$ with small values of yttrium content. We study the $\mathrm{Gd}_{2-\mathrm{x}} \mathrm{Y}_{\mathrm{x}} \mathrm{Ti}_{2} \mathrm{O}_{7}$ samples with yttrium contents $\mathrm{x}=0.1$ and 0.4 in the temperature range of 2 to $20 \mathrm{~K}$. We found that the values of the collected data and behaviour of $C_{m}$ for fields up to $2 \mathrm{~T}$ are very similar to each other and also that these values of $C_{m}$ start to decrease for fields of 3 and $4 \mathrm{~T}$. Clear in the figure is the presence 
of maximums for fields above $5 \mathrm{~T}$ which are identified as a Schottky anomalies due to separation of the energy levels in high magnetic fields. With the increasing of the field up to $9 \mathrm{~T}$, the Schottky anomaly becomes broader and the center of the peak moves to higher temperatures. We do not fit the data to the Schottky specific heat because it provides a poor fit in a similar way to the non-diluted $\mathrm{Gd}_{2} \mathrm{Ti}_{2} \mathrm{O}_{7}$ sample studied in Chapter 4 .
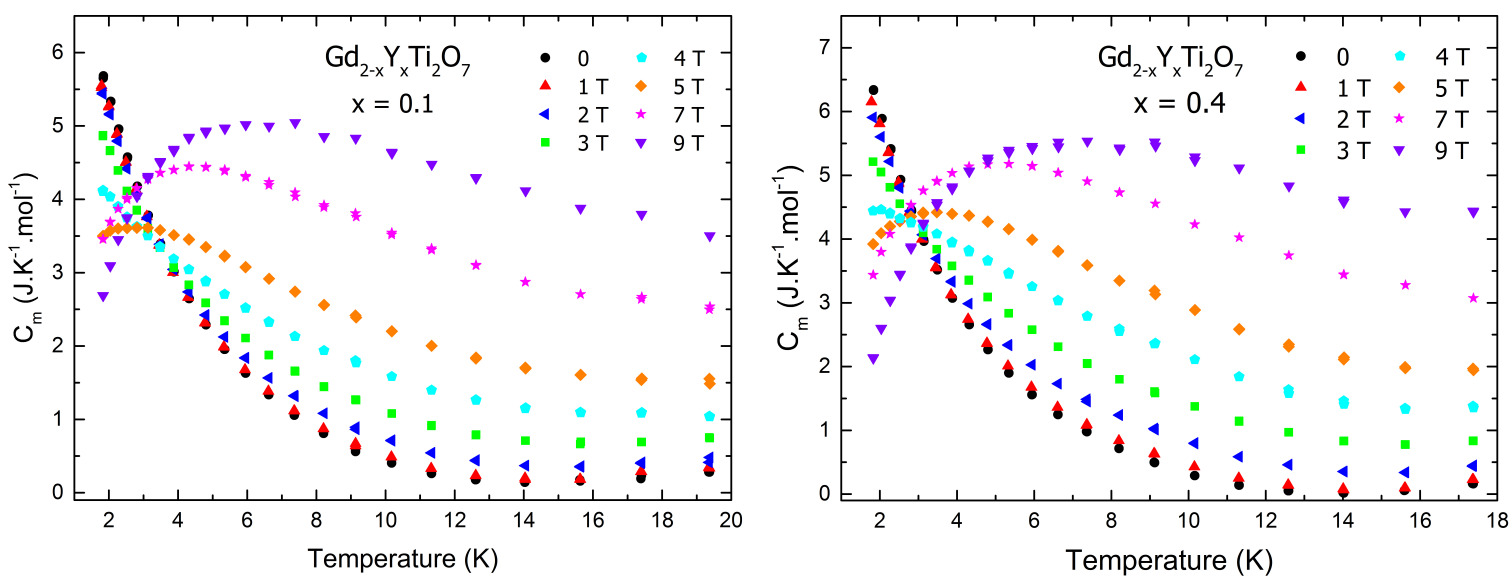

Figure 5.12: Magnetic specific heat $C_{m}$ as a function of the temperature for $\mathrm{Gd}_{2-\mathrm{x}} \mathrm{Y}_{\mathrm{x}} \mathrm{Ti}_{2} \mathrm{O}_{7}$ with yttrium contents $\mathrm{x}=0.1$ (left) and $\mathrm{x}=0.4$ (right) measured with different magnetic fields.

Figure 5.13 displays the temperature dependence of the magnetic specific heat $C_{m}$ for $\mathrm{Gd}_{2-\mathrm{x}} \mathrm{Y}_{\mathrm{x}} \mathrm{Ti}_{2} \mathrm{O}_{7}$ with yttrium contents $\mathrm{x}=0.7$ and 1 measured in different magnetic fields. On contrast with the $C_{m}$ curves above, we observe the Schottky anomaly for fields above 4 T. For $\mathrm{x}=1$, the Schottky peak is well defined at a field of $5 \mathrm{~T}$. As the field is increasing, the Schottky peak becomes broader and is centered at higher temperatures. As mentioned before, for these yttrium contents $\mathrm{x}=0.7$ and 1 the values of $C_{l a t}$ present slight differences compared with the other diluted samples. Then, if we consider different estimated values of $C_{l a t}$ we would expect that $C_{m}$ will not display that small increase close to $18 \mathrm{~K}$ but instead broader peaks better defined. 

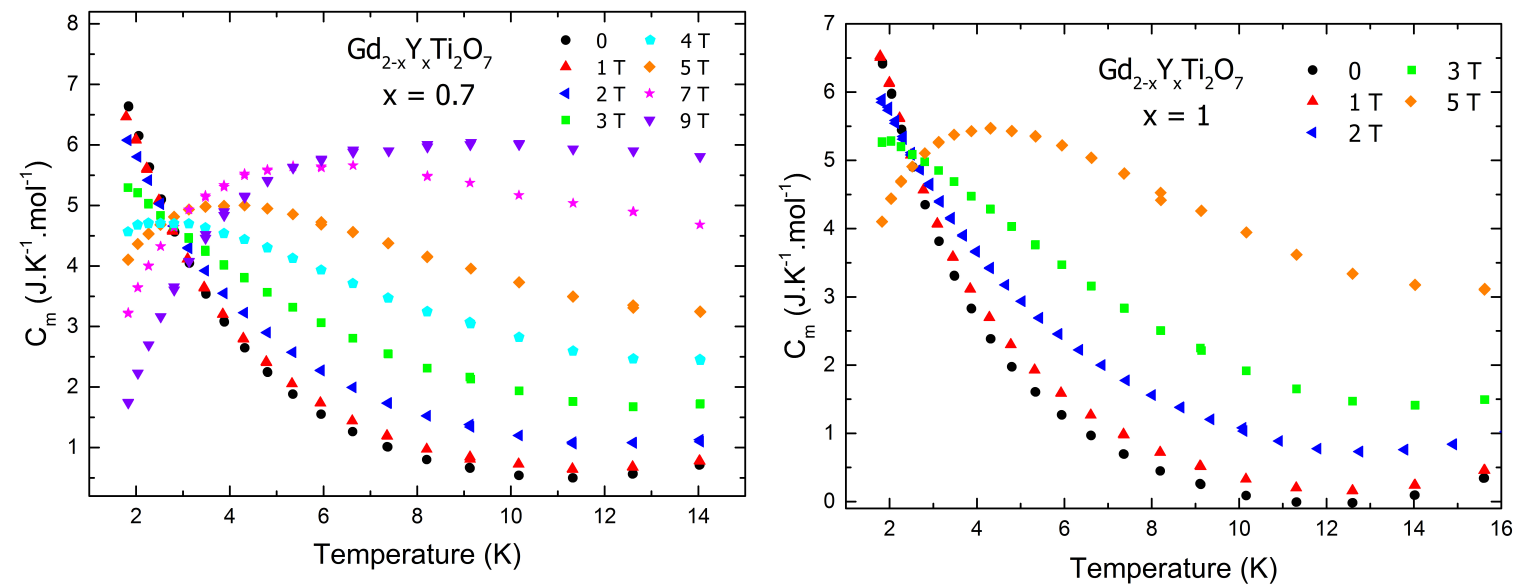

Figure 5.13: Magnetic specific heat $C_{m}$ as a function of the temperature for $\mathrm{Gd}_{2-\mathrm{x}} \mathrm{Y}_{\mathrm{x}} \mathrm{Ti}_{2} \mathrm{O}_{7}$ with yttrium contents $\mathrm{x}=0.7$ (left) and $\mathrm{x}=1$ (right) measured with different magnetic fields.

\subsection{Discussion}

The refinement of the $\mathrm{X}$-ray diffraction patterns reveal that the series of yttrium diluted $\mathrm{Gd}_{2-\mathrm{x}} \mathrm{Y}_{\mathrm{x}} \operatorname{Ti}_{2} \mathrm{O}_{7}$ also possesses a small deficiency of oxygen in the $\mathrm{O}(1)$ site in the same way that our $\mathrm{Gd}_{2} \mathrm{Ti}_{2} \mathrm{O}_{7}$ samples. The values of the occupancies of $\mathrm{O}(1)$ are very close and it seems that there is no relation between the oxygen vacancies and the yttrium content in $\operatorname{Gd}_{2-\mathrm{x}} \mathrm{Y}_{\mathrm{x}} \mathrm{Ti}_{2} \mathrm{O}_{7}$. There is a clear reduction with an almost linear dependence of the lattice parameter as the yttrium content is increased which is due to the smaller ionic radius of yttrium. So, we suppose that a distortion lattice in $\operatorname{Gd}_{2-\mathrm{x}} \mathrm{Y}_{\mathrm{x}} \mathrm{Ti}_{2} \mathrm{O}_{7}$ must consider the relaxations of the Gd and $\mathrm{Y}$ ions away from $\mathrm{O}(1)$ vacancies along $\langle 111\rangle$ directions and that it occurs in a smaller lattice.

AC susceptibility measurements of the yttrium diluted $\mathrm{Gd}_{2-\mathrm{x}} \mathrm{Y}_{\mathrm{x}} \mathrm{Ti}_{2} \mathrm{O}_{7}$ shown in figure 5.3 display peaks like phase transitions below $1 \mathrm{~K}$. By increasing the yttrium content, we found that the values of the temperature transitions decrease roughly with a linear dependence. Our curves of ac susceptibility do not display a peak corresponding to the second phase transition but for small yttrium contents; $\mathrm{x}=0.1,0.14,0,16$ and 0.2 ; the existence of a plateau after the first transition does not close this possibility. On the other hand, we do not know if for high yttrium contents in $\operatorname{Gd}_{2-\mathrm{x}} \mathrm{Y}_{\mathrm{x}} \mathrm{Ti}_{2} \mathrm{O}_{7}$ we will be able to measure a second phase transition because the peaks of the first transition are better defined as in the case of $\mathrm{x}=1$. 
Results from the Curie-Weiss fit shown in figure 5.8 display that the values of $\theta_{\mathrm{CW}}$ decreases as we increase the yttrium content in $\mathrm{Gd}_{2-\mathrm{x}} \mathrm{Y}_{\mathrm{x}} \mathrm{Ti}_{2} \mathrm{O}_{7}$ which is consistent with lower antiferromagnetic interactions. Also, the values of the effective magnetic moments $\mathrm{p}_{\text {eff }}$ oscillate around the theoretical value of $7.94 \mu_{B} / \mathrm{Gd}$ when the yttrium content is increased in $\mathrm{Gd}_{2-\mathrm{x}} \mathrm{Y}_{\mathrm{x}} \operatorname{Ti}_{2} \mathrm{O}_{7}$. We think that it could be useful to study $\mathrm{Gd}_{2-\mathrm{x}} \mathrm{Y}_{\mathrm{x}} \mathrm{Ti}_{2} \mathrm{O}_{7}$ with higher yttrium contents in order to establish better the behaviours of $\theta_{\mathrm{CW}}$ and $\mathrm{p}_{\mathrm{eff}}$.

Magnetization measurements of the $\mathrm{Gd}_{2-\mathrm{x}} \mathrm{Y}_{\mathrm{x}} \mathrm{Ti}_{2} \mathrm{O}_{7}$ samples with small yttrium content; $\mathrm{x}=0.2$, 0.3, 0.4 and 0.7; present a slight variation among them but still reach saturated moments close to the expected of $7 \mu_{B}$ for fields higher that $10 \mathrm{~T}$. For the $\mathrm{Gd}_{2-\mathrm{x}} \mathrm{Y}_{\mathrm{x}} \mathrm{Ti}_{2} \mathrm{O}_{7}$ samples with high yttrium contents such as $\mathrm{x}=1,1.1$ and 1.5, we found that they reach the same saturated moment with an applied field of $7 \mathrm{~T}$. We think that the lower values of $\theta_{\mathrm{CW}}$ and thus of exchange antiferromagnetic interactions $\mathrm{J}$ for $\mathrm{Gd}_{2-\mathrm{x}} \mathrm{Y}_{\mathrm{x}} \mathrm{Ti}_{2} \mathrm{O}_{7}$ samples with a higher yttrium content cause the fact that it is easier to reach a saturated moment since with lower antiferromagnetic interactions, the $\mathrm{Gd}_{2-\mathrm{x}} \mathrm{Y}_{\mathrm{x}} \mathrm{Ti}_{2} \mathrm{O}_{7}$ samples behave more like a paramagnet.

The magnetic specific heat of the $\mathrm{Gd}_{2-\mathrm{x}} \mathrm{Y}_{\mathrm{x}} \mathrm{Ti}_{2} \mathrm{O}_{7}$ samples as a function of the temperature and field present a Schottky anomaly above $1 \mathrm{~K}$. We found that the position of the Schottky peak shifts to higher temperatures as we increase the yttrium content. We did not fit the data to equation (41) since we know that it provides a very poor fit as discussed for $\mathrm{Gd}_{2} \mathrm{Ti}_{2} \mathrm{O}_{7}$ in Chapter 4. As in the case of $\mathrm{Gd}_{2} \mathrm{Ti}_{2} \mathrm{O}_{7}$, a better fit can be made by considering the ground state ${ }^{8} S_{7 / 2}$ with the energy levels of the crystal field. For $\mathrm{Gd}_{2-\mathrm{x}} \mathrm{Y}_{\mathrm{x}} \mathrm{Ti}_{2} \mathrm{O}_{7}$, the lack of measurements of the specific heat in a lower temperature range then we can not discuss about the first and second phase transition and compute the recovered entropy of the yttrium diluted samples. It will be interesting to study the behaviour of the transitions temperatures as we increase the yttrium content as well as to compare them with the transition temperatures obtained from ac susceptibility measurements. 


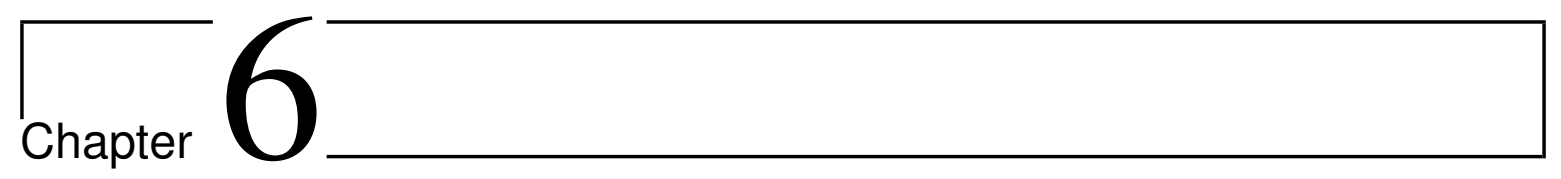

\section{Conclusion}

In this study, polycrystalline samples of $\mathrm{Gd}_{2} \mathrm{Ti}_{2} \mathrm{O}_{7}$ and yttrium-diluted $\mathrm{Gd}_{2-\mathrm{x}} \mathrm{Y}_{\mathrm{x}} \mathrm{Ti}_{2} \mathrm{O}_{7}$ were successfully synthesized by using the sol-gel method. As shown by the Rietveld refinement, our $\mathrm{Gd}_{2} \mathrm{Ti}_{2} \mathrm{O}_{7}$ samples present a varying degree of oxygen vacancies in the $\mathrm{O}(1)$ site, which can be reduced by changing the growth atmosphere of the samples to oxygen. Also, the computed lattice parameter for the sample with a better stoichiometry, that is, for $\mathrm{Gd}_{2} \mathrm{Ti}_{2} \mathrm{O}_{7}$ grown in oxygen atmosphere was found to be $10.184 \AA$. On the other side, the increasing of the yttrium content in $\operatorname{Gd}_{2-\mathrm{x}} \mathrm{Y}_{\mathrm{x}} \operatorname{Ti}_{2} \mathrm{O}_{7}$ leads to smaller lattice parameters.

Peaks of phase transition were observed close to $1 \mathrm{~K}$ through ac susceptibility measurements for $\mathrm{Gd}_{2} \mathrm{Ti}_{2} \mathrm{O}_{7}$ with annealings in oxygen, as well as for $\mathrm{Gd}_{2} \mathrm{Ti}_{2} \mathrm{O}_{7}$ grown in different atmospheres. For each yttrium-diluted sample $\mathrm{Gd}_{2-\mathrm{x}} \mathrm{Y}_{\mathrm{x}} \mathrm{Ti}_{2} \mathrm{O}_{7}$, ac susceptibility measurements show a peak of transition

below $1 \mathrm{~K}$. For small yttrium contents; $\mathrm{x}=0.1,0.14,0.16$ and 0.2 the peaks form a plateau after the first transition, which suggests that we could observe a peak corresponding to the second phase transition. For $\mathrm{x}=0.1$ and 0.7 , the well defined peaks of the first transition leave some doubts about the existence of a second phase transition. The position of the peak shifts to lower temperatures as we increase the yttrium content in $\mathrm{Gd}_{2-\mathrm{x}} \mathrm{Y}_{\mathrm{x}} \mathrm{Ti}_{2} \mathrm{O}_{7}$, then the transition temperatures decrease with a higher yttrium content.

Both $\mathrm{Gd}_{2} \mathrm{Ti}_{2} \mathrm{O}_{7}$ and $\mathrm{Gd}_{2-\mathrm{x}} \mathrm{Y}_{\mathrm{x}} \mathrm{Ti}_{2} \mathrm{O}_{7}$ samples reach a saturated moment close to $7 \mu_{B}$ for fields about $10 \mathrm{~T}$. The magnetization measurements in $\mathrm{Gd}_{2} \mathrm{Ti}_{2} \mathrm{O}_{7}$ show that the samples with less value of occupancy in the $\mathrm{O}(1)$ site, that is, with more oxygen vacancies, exhibit a small but still clear reduction in the saturated moments. In order to resolve a higher reduction in the saturated moments, 
it is required to introduce a sizeable density of oxygen vacancies. The lower values of $\theta_{\mathrm{CW}}$ and thus of exchange antiferromagnetic interactions $\mathrm{J}$ for increasing yttrium content in $\mathrm{Gd}_{2-\mathrm{x}} \mathrm{Y}_{\mathrm{x}} \mathrm{Ti}_{2} \mathrm{O}_{7}$ are responsible for the fact that it is easier to reach a saturated moment with a higher yttrium content.

Our polycrystalline $\mathrm{Gd}_{2} \mathrm{Ti}_{2} \mathrm{O}_{7}$ sample with a small deficiency of oxygen exhibits two phase transitions at the temperatures $\mathrm{T}_{\mathrm{N}}^{1}=1.04 \mathrm{~K}$ and $\mathrm{T}_{\mathrm{N}}^{2}=0.73 \mathrm{~K}$. The recovered entropy up to high temperatures of the system presents missing entropy which could be associated to the presence of oxygen vacancies. In this sample, we have discussed the $\mathrm{T}^{2}$ and $\mathrm{T}^{3}$ power laws of the magnetic specific heat, which have very similar behaviour in the temperature range $0.39 \mathrm{~K} \leq \mathrm{T} \leq 0.68 \mathrm{~K}$ studied here. In the frame of the $\mathrm{T}^{3}$ power law, for $\mathrm{Gd}_{2} \mathrm{Ti}_{2} \mathrm{O}_{7}$ we obtained the velocity of the magnons $v_{s w}$ $=46.2 \mathrm{~m} / \mathrm{s}$, which is consistent with the value $v_{s w}=45.8 \mathrm{~m} / \mathrm{s}$ reported for the antiferromagnetic pyrochlore $\mathrm{Yb}_{2} \mathrm{Ge}_{2} \mathrm{O}_{7}$ [82]. Finally, an appropriate fit to the Schottky anomaly for the $\mathrm{Gd}_{2} \mathrm{Ti}_{2} \mathrm{O}_{7}$ and $\mathrm{Gd}_{2-\mathrm{x}} \mathrm{Y}_{\mathrm{x}} \mathrm{Ti}_{2} \mathrm{O}_{7}$ samples could be made by considering the ${ }^{8} S_{7 / 2}$ state with the split of the energy levels by the crystal field.

\section{Future Work}

Future work concerns to study our $\mathrm{Gd}_{2} \mathrm{Ti}_{2} \mathrm{O}_{7}$ and $\mathrm{Gd}_{2-\mathrm{x}} \mathrm{Y}_{\mathrm{x}} \mathrm{Ti}_{2} \mathrm{O}_{7}$ samples by using high energy $\mathrm{x}$-rays at the Brazilian Synchrotron Light Laboratory (LNLS) in order to improve our structural analysis by refining the $\mathrm{x}$-ray patterns and also to confirm the same displacements of the Gd and $\mathrm{Y}$ ions as in $\mathrm{Dy}_{2} \mathrm{Ti}_{2} \mathrm{O}_{7}$. Also, in order to determine the compositions of our samples and thus the oxygen concentration or $\delta$ in $\mathrm{Gd}_{2} \mathrm{Ti}_{2} \mathrm{O}_{7-\delta}$ it is required to perform a thermogravimetric analysis.

To discuss the low-temperature behaviour of the magnetic specific heat of our samples, it is required to perform experiments below the lowest $0.39 \mathrm{~K}$ considered here. Then, for $\mathrm{Gd}_{2} \mathrm{Ti}_{2} \mathrm{O}_{7}$ studied in Chapter 4 we could find if there is any significant difference between the transition temperatures for $\mathrm{Gd}_{2} \mathrm{Ti}_{2} \mathrm{O}_{7}$ annealed in oxygen and grown in different atmospheres as well as compare the recovered magnetic entropy of our samples. Also, we could distinguish better between the $\mathrm{T}^{2}$ and $\mathrm{T}^{3}$ power laws studied in our work. For the $\mathrm{Gd}_{2-\mathrm{x}} \mathrm{Y}_{\mathrm{x}} \mathrm{Ti}_{2} \mathrm{O}_{7}$ samples, it is required to measure specific heat in a very low-temperature range in order to obtain the temperatures of the first and second phase transition. 


\section{List of References}

[1] P. Schiffer and A. P. Ramirez. Recent experimental progress in the study of geometrical magnetic frustration. Comments on Condensed Matter Physics 18, 21-50 (1996).

[2] J. S. Gardner, M. J. P. Gingras and J. E. Greedan. Magnetic pyrochlore oxides. Reviews of Modern Physics 82, 53-66 (2010).

[3] S. Andergassen. A frustrated trio. Nature 495, 321-322 (2013).

[4] A. S. Wills, M. E. Zhitomirsky, B. Canals, J. P. Sanchez, P. Bonville, P. Dalmas de Réotier and A. Yaouanc. Magnetic ordering in $\mathrm{Gd}_{2} \mathrm{Sn}_{2} \mathrm{O}_{7}$ : the archetypal Heisenberg pyrochlore antiferromagnet. Journal of Physics: Condensed Matter 18, L37-L42 (2006).

[5] A. P. Ramirez. Geometrically frustrated matter-magnets to molecules. Materials Research Society Bulletin 30, 447-451 (2005).

[6] C. Lacroix, P. Mendels, and F. Mila. Introduction to frustrated magnetism. Springer Series in Solid-State Sciences, Heidelberg, 3-22 (2011).

[7] J. E. Greedan. Frustrated rare earth magnetism: Spin glasses, spin liquids and spin ices in pyrochlore oxides. Journal of Alloys and Compounds 408-412, 444-455 (2006).

[8] S. Blundell. Magnetism in condensed matter. Oxford University Press, New York (2001).

[9] C. Kittel. Introduction to solid state physics. John Wiley \& Sons, Inc. United States of America, eighth edition (2005). 
[10] N. W. Ashcroft and N. D. Mermin. Solid state physics. Holt, Rinehart and Winston, New York (1976).

[11] P. Dalmas de Réotier, A. Yaouanc, Y. Chapuis, S. H. Curnoe, B. Grenier, E. Ressouche, C. Marin, J. Lago, C. Baines and S. R. Giblin. Magnetic order, magnetic correlations, and spin dynamics in the pyrochlore antiferromagnet $\mathrm{Er}_{2} \mathrm{Ti}_{2} \mathrm{O}_{7}$. Physical Review B 86, 104424 (2012).

[12] O. V. Lounasmaa, A. J. Freeman and R. B. Frankel. Nuclear specific heats in metals and alloys. Hyperfine Interactions. New York and London, Academic Press, 467-496 (1967).

[13] E. S. R. Gopal. Specific heats at low temperatures. The International Cryogenics Monograph Series. Springer US, first edition (1966).

[14] S. Petit, S. Guitteny, J. Robert, P. Bonville, C. Decorse, J. Ollivier, H. Mutka and I. Mirebeau. Spin dynamics in highly frustrated pyrochlore magnets. European Physical Journal Web of Conferences 83, 03012 (2015).

[15] A. P. Ramirez, B. S. Shastry, A. Hayashi, J. J. Krajewski, D. A. Huse and R. J. Cava. Multiple field-induced phase transitions in the geometrically frustrated dipolar magnet: $\mathrm{Gd}_{2} \mathrm{Ti}_{2} \mathrm{O}_{7}$. Physical Review Letters 89, 0672021-0672024 (2002).

[16] A. P. Ramirez. Strongly geometrically frustrated magnets. Annual Review of Materials Science 24, 453-480 (1994).

[17] L. D. C. Jaubert and P. C. W. Holdsworth. Magnetic monopole dynamics in spin ice. Journal of Physics: Condensed Matter 23, 164222 (2011).

[18] N. P. Raju, M. Dion, M. J. P. Gingras and T. E. Mason. Transition to long-range magnetic order in the highly frustrated insulating pyrochlore antiferromagnet $\mathrm{Gd}_{2} \mathrm{Ti}_{2} \mathrm{O}_{7}$. Physical Review B 59, 14489-14498 (1999).

[19] M. J. Harris, S. T. Bramwell, D. F. McMorrow, T. Zeiske, and K. W. Godfrey. Geometrical frustration in the ferromagnetic pyrochlore $\mathrm{Ho}_{2} \mathrm{Ti}_{2} \mathrm{O}_{7}$. Physical Review Letters 79, 13, 2554 (1997).

[20] S. T. Bramwell, M. J. Harris. Frustration in Ising-type spin models on the pyrochlore lattice. Journal of Physics: Condensed Matter 10, 215-220 (1998).

[21] L. Pauling. The structure and entropy of ice and of other crystals with some randomness of atomic arrangement. Journal of the American Chemical Society 57, 2680-2684 (1935). 
[22] D. J. Flood. Magnetization and magnetic entropy of $\mathrm{Dy}_{2} \mathrm{Ti}_{2} \mathrm{O}_{7}$. Journal of Applied Physics 45, 4041-4044 (1974).

[23] Y. M. Jana, A. Sengupta and D. Ghosh. Journal of Magnetism and Magnetic Materials 248, $7-18(2002)$.

[24] A. P. Ramirez, A. Hayashi, R. J. Cava, R. Siddharthan and B. S. Shastry. Zero-point entropy in "spin ice". Nature 399, 333-335 (1999).

[25] B. C. den Hertog and M. J. P. Gingras. Dipolar interactions and origin of spin ice in Ising pyrochlore magnets. Physical Review Letters 84, 3430-3433 (2000).

[26] S. T. Bramwell, M. J. Harris, B. C. den Hertog, M. J. P. Gingras, J. S. Gardner, D. F. McMorrow, A. R. Wildes, A. L. Cornelius, J. D. M. Champion, R. G. Melko, and T. Fennell. Spin Correlations in $\mathrm{Ho}_{2} \mathrm{Ti}_{2} \mathrm{O}_{7}$ : A Dipolar Spin Ice System. Physical Review Letters 87, 4, 0472051-0472054 (2001).

[27] L. Balents. Spin liquids in frustrated magnets. Nature 464, 199-208 (2010).

[28] P.W. Anderson. Resonating valence bonds: A new kind of insulator?. Materials Research Bulletin 8, 153-160 (1973).

[29] M. J. P. Gingras, B. C. den Hertog, M. Faucher, J. S. Gardner, S. R. Dunsiger, L. J. Chang, B. D. Gaulin, N. P. Raju, and J. E. Greedan. Thermodynamic and single-ion properties of $\mathrm{Tb}^{3+}$ within the collective paramagnetic-spin liquid state of the frustrated pyrochlore antiferromagnet $\mathrm{Tb}_{2} \mathrm{Ti}_{2} \mathrm{O}_{7}$. Physical Review B 62, 10, 6496-6511 (2000).

[30] J. S. Gardner, S. R. Dunsiger, B. D. Gaulin, M. J. P. Gingras, J. E. Greedan, R. F. Kiefl, M. D. Lumsden, W. A. MacFarlane, N. P. Raju, J. E. Sonier, I. Swainson, and Z. Tun. Cooperative paramagnetism in the geometrically frustrated pyrochlore antiferromagnet $\mathrm{Tb}_{2} \mathrm{Ti}_{2} \mathrm{O}_{7}$. Physical Review Letters 82, 1012-1015 (1999).

[31] Y. Yasui, M. Kanada, M. Ito, H. Harashina, M. Sato, H. Okumura, K. Kakurai, and H. J. Kadowaki. Static correlation and dynamical properties of $\mathrm{Tb}^{3+}$ moments in $\mathrm{Tb}_{2} \mathrm{Ti}_{2} \mathrm{O}_{7}-$ Neutron scattering study. Journal of the Physical Society of Japan 71, 599-621 (2002).

[32] J. S. Gardner, A. Keren, G. Ehlers, C. Stock, Eva Segal, J. M. Roper, B. Fäk, M. B. Stone, P. R. Hammar, D. H. Reich and B. D. Gaulin. Dynamic frustrated magnetism in $\mathrm{Tb}_{2} \mathrm{Ti}_{2} \mathrm{O}_{7}$ at 50 mK. Physical Review B 68, 1804011-1804014 (2003). 
[33] H. R. Molavian, M. J. P. Gingras, and B. Canals. Dynamically induced frustration as a route to a quantum spin ice state in $\mathrm{Tb}_{2} \mathrm{Ti}_{2} \mathrm{O}_{7}$ via virtual crystal field excitations and quantum many-body effects. Physical Review Letters 98, 1572041-1572044 (2007).

[34] H. W. J. Blöte, R. F. Wielinga, W. J. Huiskamp. Heat-capacity measurements on rare-earth double oxides $\mathrm{R}_{2} \mathrm{M}_{2} \mathrm{O}_{7}$. Physica 43, 549-568 (1969).

[35] S. T. Bramwell, M. N. Field, M. J. Harris and I. P. Parkin. Magnetization of the heavy rare earth titanate pyrochlores-a series of model frustrated magnets. Journal of Physics: Condensed Matter 12, 483-495 (2000).

[36] J. A. Hodges, P. Bonville, A. Forget, A. Yaouanc, P. Dalmas de Réotier, G. André, M. Rams, K. Królas, C. Ritter, P. C. M. Gubbens, C. T. Kaiser, P. J. C. King, and C. Baines. First-order transition in the spin dynamics of geometrically frustrated $\mathrm{Yb}_{2} \mathrm{Ti}_{2} \mathrm{O}_{7}$. Physical Review Letters 88, 0772041-0772044 (2002).

[37] K. A. Ross, L. R. Yaraskavitch, M. Laver, J. S. Gardner, J. A. Quilliam, S. Meng, J. B. Kycia, D. K. Singh, Th. Proffen, H. A. Dabkowska, and B. D. Gaulin. Dimensional evolution of spin correlations in the magnetic pyrochlore $\mathrm{Yb}_{2} \mathrm{Ti}_{2} \mathrm{O}_{7}$. Physical Review B 84, 1744421-1744426 (2011).

[38] K. A. Ross, Th. Proffen, H. A. Dabkowska, J. A. Quilliam, L. R. Yaraskavitch, J. B. Kycia and B.D. Gaulin. Physical Review B 86, 1744241-17442411 (2013).

[39] P. Bonville, J. A. Hodges, M. Ocio, J. P. Sanchez, P. Vulliet, S. Sosin and D. Braithwaite. Low temperature magnetic properties of geometrically frustrated $\mathrm{Gd}_{2} \mathrm{Sn}_{2} \mathrm{O}_{7}$ and $\mathrm{Gd}_{2} \mathrm{Ti}_{2} \mathrm{O}_{7}$. Journal of Physics: Condensed Matter 15, 7777-7787 (2003).

[40] J. R. Stewart, G. Ehlers, A. S. Wills, S. T. Bramwell and J. S. Gardner. Phase transitions, partial disorder and multi-k structures in $\mathrm{Gd}_{2} \mathrm{Ti}_{2} \mathrm{O}_{7}$. Journal of Physics: Condensed Matter 16, L321-L326 (2004).

[41] H. Fukazawa, R. G. Melko, R. Higashinaka, Y. Maeno and M. J. P. Gingras. Magnetic anisotropy of the spin-ice compound $\mathrm{Dy}_{2} \mathrm{Ti}_{2} \mathrm{O}_{7}$. Physical Review B 65, 054410 (2002).

[42] M. J. Harris, S. T. Bramwell, T. Zeiske, D. F. McMorrow and P. J. C. King. Magnetic structures of highly frustrated pyrochlores. Journal of Magnetism and Magnetic Materials 177-181, 757-762 (1998). 
[43] J. D. M. Champion, M. J. Harris, P. C. W. Holdsworth, A. S. Wills, G. Balakrishnan, S. T. Bramwell, E. Cizmár, T. Fennell, J. S. Gardner, J. Lago, D. F. McMorrow, M. Orendác, A. Orendácová, D. McK. Paul, R. I. Smith, M. T. F. Telling, and A. Wildes. $\operatorname{Er}_{2} \mathrm{Ti}_{2} \mathrm{O}_{7}$ : Evidence of quantum order by disorder in a frustrated antiferromagnet. Physical Review B 68, 020401 (2003).

[44] L. Chang, S. Onoda, Y. Su, Y. Kao, K. Tsuei, Y. Yasui, K. Kakurai and M. R. Lees. Higgs transition from a magnetic Coulomb liquid to a ferromagnet in $\mathrm{Yb}_{2} \mathrm{Ti}_{2} \mathrm{O}_{7}$. Nature Communications 3992 (2012).

[45] K. A. Ross, J. P. C. Ruff, C. P. Adams, J. S. Gardner, H. A. Dabkowska, Y. Qiu, J. R. D. Copley, and B. D. Gaulin. Two-dimensional Kagomé correlations and field induced order in the ferromagnetic XY pyrochlore $\mathrm{Yb}_{2} \mathrm{Ti}_{2} \mathrm{O}_{7}$. Physical Review Letters 103, 227202 (2009).

[46] V. N. Glazkov, M. E. Zhitomirsky, A. I. Smirnov, H.-A. Krug von Nidda, A. Loidl, C. Marin, and J. P. Sanchez. Single-ion anisotropy in the gadolinium pyrochlores studied by electron paramagnetic resonance. Physical Review B 72, 020409 (2005).

[47] V. N. Glazkov, M. E. Zhitomirsky, A. I. Smirnov, C. Marin, J. P. Sanchez, A. Forget, D. Colson and P. Bonville. Single-ion anisotropy and transverse magnetization in the frustrated gadolinium pyrochlores. Journal of Physics: Condensed Matter 19, 145271 (2007).

[48] V. N. Glazkov, C. Marin and J. P. Sanchez. Observation of a transverse magnetization in the ordered phases of the pyrochlore magnet $\mathrm{Gd}_{2} \mathrm{Ti}_{2} \mathrm{O}_{7}$. Journal of Physics: Condensed Matter 18, L429-L434 (2006).

[49] O. A. Petrenko, M. R. Lees and G. Balakrishnan. Titanium pyrochlore magnets: How much can be learned from magnetization measurements?. Journal of Physics: Condensed Matter 23, 164218 (2011).

[50] J. D. M. Champion, A. S. Wills, T. Fennell, S. T. Bramwell, J. S. Gardner and M. A. Green. Order in the Heisenberg pyrochlore: the magnetic structure of $\mathrm{Gd}_{2} \mathrm{Ti}_{2} \mathrm{O}_{7}$. Physical Review B 64, 1404071-1404074 (2001).

[51] O. A. Petrenko, M. R. Lees, G. Balakrishnan and D. McK Paul. Magnetic phase diagram of the antiferromagnetic pyrochlore $\mathrm{Gd}_{2} \mathrm{Ti}_{2} \mathrm{O}_{7}$. Physical Review B 70, 0124021-0124024 (2004). 
[52] R. D'Ortenzio. Ground state of the quantum spin ice $\mathrm{Yb}_{2} \mathrm{Ti}_{2} \mathrm{O}_{7}$. Open Access Dissertations and Theses, 8424 (2013).

[53] P. W. Anderson. Ordering and antiferromagnetism in ferrites. Physical Review 102, 1008-1013 (1956).

[54] J. Villain. Insulating spin glasses. Zeitschrift für Physik B 33, 31-42 (1979).

[55] J. N. Reimers, A. J. Berlinsky, and A. C. Shi. Mean-field approach to magnetic ordering in highly frustrated pyrochlores. Physical Review B 43, 865-878 (1991).

[56] J. N. Reimers and A. J. Berlinsky. Order by disorder in the classical Heisenberg Kagomé antiferromagnet. Physical Review B 48, 9539-9554 (1993).

[57] S. E. Palmer and J. T. Chalker. Order induced by dipolar interactions in a geometrically frustrated antiferromagnet. Physical Review B 62, 14488-14492 (2000).

[58] O. Cépas, A. P. Young and B. S. Shastry. Degeneracy and strong fluctuation-induced first-order phase transition in the dipolar pyrochlore antiferromagnet. Physical Review B 72, 18440811844087 (2005).

[59] J. Frunzke, T. Hansen, A. Harrison, J. S. Lord, G. S. Oakley, D. Visserc, and A. S. Wills. Magnetic ordering in diluted Kagomé antiferromagnets. Journal of Materials Chemistry 11, 179-185 (2001).

[60] O. A. Petrenko, M. R. Lees, G. Balakrishnan, V. N. Glazkov and S. S. Sosin. Magnetic phases in a $\mathrm{Gd}_{2} \mathrm{Ti}_{2} \mathrm{O}_{7}$ pyrochlore for a field applied along the [100] axis. Physical Review B 85, 18041211804124 (2012).

[61] J. A. M. Paddison, A. B. Cairns, D. D. Khalyavin, P. Manuel, A. Daoud-Aladine, G. Ehlers, O. A. Petrenko, J. S. Gardner, H. D. Zhou, A. L. Goodwin, and J. R. Stewart. Nature of partial magnetic order in the frustrated antiferromagnetic $\mathrm{Gd}_{2} \mathrm{Ti}_{2} \mathrm{O}_{7}$. arXiv:1506.05045. 1-5 (2015).

[62] B. Javanparast, Z. Hao, M. Enjalran and M. J. P. Gingras. Fluctuation-driven selection at critically in a frustrated magnetic system: the case of multiple- $k$ partial order on the pyrochlore lattice. Physical Review Letters 114, 130601 (2015).

[63] G. Sala, M. J. Gutmann, D. Prabhakaran, D. Pomaranski, C. Mitchelitis and J. B. Kycia. Vacancy defects and monopole dynamics in oxygen-deficient pyrochlores. Nature Materials 13, 488-493 (2014). 
[64] G. Sala, M. J. Gutmann, D. Prabhakaran, D. Pomaranski, C. Mitchelitis and J. B. Kycia. Supplementary Information, Vacancy defects and monopole dynamics in oxygen-deficient pyrochlores. Nature Materials 13, section C (2014).

[65] G. Balakrishnan, O. A. Petrenko, M. R. Lees and D. McK Paul. Single crystal growth of rare earth titanate pyrochlores. Journal of Physics: Condensed Matter 10, L723-L725 (1998).

[66] Z. S. Chen, W. P. Gong, T. F. Chen and S. L. Li. Synthesis and characterization of pyrochloretype yttrium titanate nanoparticles by modified sol-gel method. Bulletin of Materials Science 34, 3, 429-434 (2011).

[67] A. Garbout, S. Bouattour and A.W. Kolsi. Sol-gel synthesis, structure characterization and Raman spectroscopy of $\mathrm{Gd}_{2-2 x} \mathrm{Bi}_{2 x} \mathrm{Ti}_{2} \mathrm{O}_{7}$ solid solutions. Journal of Alloys and Compounds 469, 229-236 (2009).

[68] A. Garbout, S. Bouattour, M. Ellouze and A.W. Kolsi. Synthesis, FT-IR and X-ray diffraction investigations of gadolinium-substituted pyrochlore oxide $\mathrm{Gd}_{1.82} \mathrm{Cs}_{0.18} \mathrm{Ti}_{2} \mathrm{O}_{6.82}$ via a sol-gel process. Journal of Alloys and Compounds 425, 88-95 (2006).

[69] S. A. Speakman. Basics of X-ray powder diffraction. Training to Become an Independent User of the X-Ray SEF at the Center for Materials Science and Engineering at MIT. Massachusetts Institute of Technology.

[70] A. N. Radhakrishnan, P. Prabhakar Rao, K. S. Mary Linsa, M. Deepa and Peter Koshy. Influence of disorder-to-order transition on lattice thermal expansion and oxide ion conductivity in $\left(\mathrm{Ca}_{x} \mathrm{Gd}_{1-x}\right)_{2}\left(\mathrm{Zr}_{1-x} \mathrm{M}_{x}\right)_{2} \mathrm{O}_{7}$ pyrochlore solid solutions. Dalton Transactions 40, 3839 (2011).

[71] J. Rodríguez-Carvajal. Recent advances in magnetic structure determination by neutron powder diffraction. Physica B 192, 55-69 (1993).

[72] H. M. Rietveld. Refinement method for nuclear and magnetic structures. Journal of Applied Crystallography 2, 65-71 (1969).

[73] S. Foner. Versatile and sensitive vibrating-sample magnetometer. The Review of Scientific Instruments 30, 7, 548-557 (1959).

[74] M. Nikolo. Superconductivity: A guide to alternating current susceptibility measurements and alternating current susceptometer design. American Journal of Physics 63, 57, 57-65 (1995). 
[75] Magnetic Property Measurement System, SQUID VSM User's Manual. Quantum Design, 17th edition, San Diego (2009).

[76] J. Bardeen, L. N. Cooper and J. R. Schrieffer. Theory of Superconductivity. Physical Review 108, 5, 1175-1204 (1957).

[77] M. Tinkham. Introduction to superconductivity. McGraw-Hill, Inc., 2nd edition (1996).

[78] Physical Property Measurement System, Heat Capacity Option User's Manual. Quantum Design, 17th edition, San Diego (2010).

[79] A. Yaouanc, P. Dalmas de Réotier, V. Glazkov, C. Marin, P. Bonville, J. A. Hodges, P. C. M. Gubbens, S. Sakarya and C. Baines. Magnetic density of states at low energy in geometrically frustrated systems. Physical Review Letters 95, 047203 (2005).

[80] J. A. Quilliam, K.A. Ross, A.G. Del Maestro, M.J.P. Gingras, L.R. Corruccini and J.B. Kycia. Evidence for gapped spin-wave excitations in the frustrated $\mathrm{Gd}_{2} \mathrm{Sn}_{2} \mathrm{O}_{7}$ pyrochlore antiferromagnet from low-temperature specific heat measurements. Physical Review Letters 99, 097201 (2007).

[81] F. B. Zhang, Q. J. Li, Z. Y. Zhao, C. Fan, S. J. Li, X. G. Liu, X. Zhao, and X. F. Sun. Lowtemperature heat transport in the geometrically frustrated antiferromagnets $\mathrm{R}_{2} \mathrm{Ti}_{2} \mathrm{O}_{7}(\mathrm{R}=\mathrm{Gd}$ and Er). Physical Review B 89, 094403 (2014).

[82] Z. L. Dun, X. Li, R. S. Freitas, E. Arrighi, C. R. Dela Cruz, M. Lee, E. S. Choi, H. B. Cao, H. J. Silverstein, C. R. Wiebe, J. G. Cheng and H. D. Zhou. Antiferromagnetic order in the pyrochlores $\mathrm{R}_{2} \mathrm{Ge}_{2} \mathrm{O}_{7}(\mathrm{R}=\mathrm{Er}, \mathrm{Yb})$. Physical Review B 92, 140407 (2015).

[83] L. Savary, K. A. Ross, B. D. Gaulin, J. P. C. Ruff and L. Balents. Order by quantum disorder in $\mathrm{Er}_{2} \mathrm{Ti}_{2} \mathrm{O}_{7}$. Physical Review Letters 109, 167201 (2012). 